AMBIGUITIES IN

ONE-DIMENSIONAL PHASE

RETRIEVAL FROM FOURIER MAGNITUDES 



\title{
Ambiguities in one-dimensional phase retrieval from FourIER magnitudes
}

\section{Dissertation}

\author{
zur Erlangung des \\ mathematisch-naturwissenschaftlichen Doktorgrades \\ „DOCTOR RERUM NATURALIUM“ \\ der Georg-August-Universität Göttingen \\ im Promotionsstudiengang Mathematical Sciences \\ der Georg August University School of Science (GAUSS)
}

vorgelegt von

\section{Robert BEINERT}

aus Malchin 


\section{Betreuungsausschuss}

Prof. Dr. Gerlind Plonka-Hoch (NAM $\left.{ }^{1}\right)$

Prof. Dr. D. Russell LuKe (NAM')

\section{Mitglieder der Prüfungskommission}

Referentin: $\quad$ Prof. Dr. Gerlind Plonka-Hoch (NAM')

Korreferent: $\quad$ Prof. Dr. Gabriele StEIDL ${ }^{4}$

Weitere Mitglieder: Prof. Dr. Dorothea Bahns $\left(\mathrm{MI}^{2}\right)$

Prof. Dr. Stephan Huckemann (IMS 3 )

Prof. Dr. D. Russell LuKe (NAM $\left.{ }^{1}\right)$

Prof. Dr. Anita Schöвel (NAM')

\section{Weitere auswärtige Gutachter}

Prof. Dr. Stefan Kunis ${ }^{5}$

'Institut für Numerische und Angewandte Mathematik,

${ }^{2}$ Mathematisches Institut,

3Institut für Mathematische Stochastik,

(jeweils Fakultät für Mathematik und Informatik, Georg-August-Universität Göttingen)

${ }^{4}$ Felix-KLEIN-Zentrum für Mathematik,

(Fachbereich Mathematik, Technische Universität Kaiserslautern)

${ }^{5}$ Institut für Mathematik,

(Fachbereich 6 Mathematik/Informatik, Universität Osnabrück)

Tag der mündlichen Prüfung: 16. Dezember 2015 
To the lights who lead me through the darkest night. 

Wherefore I perceive that there is nothing better, than that a man should rejoice in his own works; for that is his portion.

Ecclesiastes 3,22

So sah ich denn, dass nichts Besseres ist, als dass ein Mensch fröhlich sei in seiner Arbeit; denn das ist sein Teil.

Prediger Salomo 3,22 

\begin{tabular}{ll}
\hline Abstract \\
\hline
\end{tabular}

In many scientific areas, such as astronomy, electron microscopy, and crystallography, one is faced with the problem to recover an unknown signal from the magnitudes of its FourIER transform. Unfortunately, this phase retrieval problem is complicated by the well-known ambiguousness. In order to find the original signal within the solution set, one hence requires further information about the unknown signal. The dissertation on hand aims to characterize the complete solution set of the one-dimensional phase retrieval problem and to investigate how far additional data or a priori conditions can ensure uniqueness.

For this purpose, we firstly restrict ourselves to the recovery of complexvalued discrete-time signals with finite support. Using a novel approach, we here give a complete characterization of all occurring ambiguities. Moreover, we show that each further solution of the discrete-time phase retrieval problem can be described by an appropriate convolution representation of the original signal and by suitable rotations, shifts, and conjugations and reflections of the appearing factors.

Using our characterization of the solution set, we investigate different a priori condition in order to reduce the number of ambiguities or even to receive a unique solution. Firstly, we consider the assumption that the unknown signal only possesses real and non-negative components. Although this can avoid the appearance of ambiguities in certain cases, the non-negativity cannot ensure uniqueness in general. Further, if we have access to additional magnitudes or phases of the unknown signal in the time domain, we can show that almost all signals with finite support can be uniquely recovered. An analogous result can be obtained by exploiting additional interference measurements. Here we study the interference of the unknown signal with a known or an unknown reference and with modulations of the signal itself.

Furthermore, we analyse the continuous-time phase retrieval problem. If the unknown signal possesses a specific structure, we can here transfer most of our previous findings. Based on this observation, we study the relation between 
the continuous-time and discrete-time problem. For arbitrary continuous-time signals, we can again avoid undesirable ambiguities by employing appropriate interference measurements.

Finally, we consider the recovery of an unknown signal from its FrESNEL magnitudes. Here the complete solution set can be characterized similarly to the FOURIER setting. Moreover, we transfer most of the ideas to enforce uniqueness by using the close relation between the FourIER and Fresnel transform. 


\section{Acknowledgement}

Looking back at the last two and a half years, I would like to thank everyone who made this thesis possible.

Foremost, I am indebted to my supervisor Gerlind PLONKA-HocH for her admirable support, enthusiasm, and encouragement, which helped me to find the road again if it seemed to be lost, and for the always open door. Moreover, I thank Russell Luke for being my co-supervisor and for asking questions, which let me think outside the box, and Gabriele STEIDL and Stefan KunIs for taking the positions as co-referees without hesitation.

I am thankful for the possibility to research as a member of the Collaborative Research Center (SFB 755) Nanoscale Photonic Imaging and later of the Research Training Group (GRK 2088) Discovering structure in complex data: Statistics meets Optimization and Inverse Problems and gratefully acknowledge the financial support through the German Research Foundation (DFG).

Moreover, I am glad for the travel opportunities, which enabled me to visit several conferences and workshops. In this context, my thanks especially go to Stefan KunIs and Gabriele STEIDL for inviting me to hold presentations and for giving me new inspirations for further studies.

Besides, I owe thanks to all my (former) colleagues from the Mathematical Signal and Image Processing group and from the 'extended research group' for the pleasant working environment. Especially, I would like to mention Marius WischerHoff and David JAMEs for many fruitful and merry discussions and for sharing the rough and the smooth.

For proofreading this thesis, I highly appreciate the help of David JamEs, Chris LAppe, and Pär Persson Mattsson. Finally, I would like to thank my parents, grant parents, and friends for their unconditional support during my entire student days. Thank you for everything! 



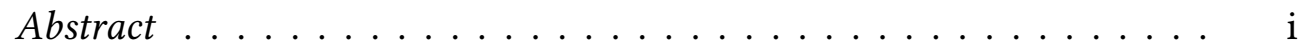

Acknowledgement ................... iii

List of figures $\ldots \ldots \ldots \ldots \ldots \ldots \ldots \ldots$ vii

Introduction

I. Characterization of the arising ambiguities 9

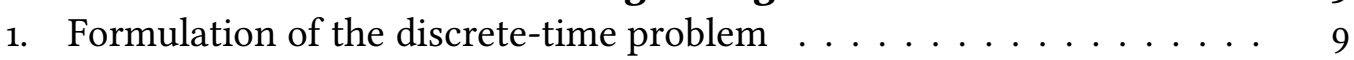

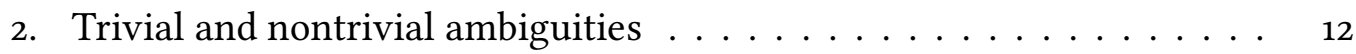

3. The phase retrieval problem in the frequency domain . . . . . . 20

4. Finding roots of a trigonometric polynomial . . . . . . . . . . . 24

4.1. Examining the zero set of the associated polynomial . . . . . . . 24

4.2. Determining the trigonometric root polynomial . . . . . . . . 29

5. Retransformation into the time domain . . . . . . . . . . . . 34

6. Number of non-trivial ambiguities . . . . . . . . . . . . . 40 40

II. Phase retrieval of non-negative signals 49

7. Non-negativity of a real signal . . . . . . . . . . . . . . . . . . 49

8. Positivity of algebraic polynomials $\ldots \ldots \ldots \ldots 53$

8.1. Dependency on the corresponding zero set . . . . . . . . . . 54

8.2. Constraints for the last conjugate zero pair . . . . . . . . . . 57

9. Non-negative solutions of the phase retrieval problem . . . . . . . . . 64

9.1. Non-negativity of non-trivially different ambiguities . . . . . . . 64

9.2. Continuity of the phase retrieval problem . . . . . . . . 72

III. Additional information in the time domain $\quad 81$

10. Using additional magnitudes of the unknown signal . . . . . . . . . 81

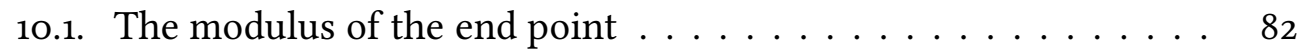

10.2. The modulus of an arbitrary signal value . . . . . . . . . 88 
10.3. The moduli of the entire signal _ . . . . . . . . . . . . . 95

11. Using additional phase information . . . . . . . . . 100

11.1. Phase of an arbitrary point and the end point . . . . . . . . 101

11.2. Phase of two arbitrary points . . . . . . . . . . . . . 109

11.3. The phase of the entire signal . . . . . . . . . . . 122

12. Using additionally given points of the signal . . . . . . . . . 124

IV. Enforcing uniqueness by interference measurements $\quad 127$

13. Interference with a known reference signal . . . . . . . . . . . . . . . 127

13.1. Interference with a known Dirac signal . . . . . . . . . . 128

13.2. Interference with a known reference signal . . . . . . . . . 131

14. Interference with an unknown reference signal . . . . . . . . . . . 138

15. Interference with a modulated version of the unknown signal itself . . 149

15.1. Phase reconstruction by using a polarization identity . . . . . 150

15.2. Reducing the number of required interference measurements . . 157

V. Continuous-time phase retrieval $\quad 163$

16. Recovery of structured continuous-time signals . . . . . . . . . . 163

17. Avoiding ambiguousness in the phase retrieval of structured signals . 170

18. Phase retrieval of arbitrary continuous-time signals . . . . . . . . . 179

19. The relation between the discrete-time and continuous-time problem . 187

2o. Ensuring uniqueness in the continuous-time phase retrieval . . . . . 189

VI. Phase retrieval from Fresnel magnitudes 199

21. The Fresnel transform . . . . . . . . . . . . . . . . . . . . . . 199

22. Ambiguities of the Fresnel phase retrieval problem . . . . . . . . . . 203

23. Establishing a Fresnel convolution theorem . . . . . . . . . . . 209

24. Phase retrieval of structured signals from Fresnel magnitudes . . . . 215

$\begin{array}{ll}\text { Conclusion and Outlook } & 217\end{array}$

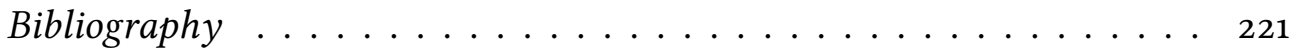

Glossary of symbols . . . . . . . . . . . . . . . . . . . . . . . . . . . . . . . . . . . 227

Index . . . . . . . . . . . . . . . . . . . . . . 229

Curriculum vitae . . . . . . . . . . . . . . . . . 237 
I. Characterization of the arising ambiguities

1.1. Discrete-time signal with compact support and corresponding FouR-

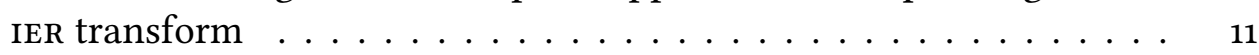

2.1. Ambiguities caused by rotation, time shift, and reflection and conjugation in the discrete-time phase retrieval problem . . . . . . .

2.2. Non-trivial ambiguities of the discrete-time phase retrieval problem caused by convolution . . . . . . . . . . . . . 18

6.1. Discrete-time phase retrieval problem with a unique solution up to trivial ambiguities . . . . . . . . . . . . . . . 44

6.2. Discrete-time phase retrieval problem with exactly three non-trivial

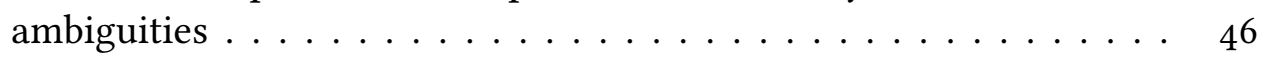

6.3. Discrete-time phase retrieval problem with a full solution set of $2^{8}$ non-trivial ambiguities . . . . . . . . . . . . . 47

\section{Phase retrieval of non-negative signals}

7.1. Discrete-time phase retrieval problem of non-negative signals with a unique solution up to shifts . . . . . . . . . . . 51

7.2. Discrete-time phase retrieval problem of non-negative signals with a full solution set of $2^{4}$ non-trivial ambiguities . . . . . . . . 52

7.3. Discrete-time phase retrieval problem of real-valued signals without a non-negative solution . . . . . . . . . . . . 52

8.1. Restriction on the last zero pair in order to ensure positivity of the corresponding monic polynomial . . . . . . . . . . . 60

8.2. Restriction on the last zero pair in order to ensure positivity of the corresponding monic polynomial for fixed zeros with negative real

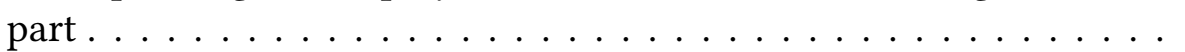


9.1. Restriction on the last zero pair in order to ensure non-negativity of different non-trivial ambiguities . . . . . . . . . . . 67

\section{Additional information in the time domain}

10.1. Discrete-time phase retrieval problem to recover the signal $x$ from its FOURIER intensity and its moduli with two further non-trivial am-

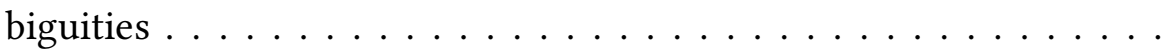

IV. Enforcing uniqueness by interference measurements

13.1. Discrete-time phase retrieval problem to recover the signal $x$ from the FourIER intensities $\mathscr{F}[x]$ and $\mathscr{F}[x+h]$ for a known reference signal $h$ with a second non-trivial solution . . . . . . . . . . 137

14.1. Discrete-time phase retrieval problem to recover the signals $x$ and $h$ from the FourIER intensities $\mathscr{F}[x], \mathcal{F}[h]$, and $\mathscr{F}[x+h]$ with at least one further non-trivially different solution pair $\breve{x}$ and $\breve{h} \ldots \ldots \ldots 148$

V. Continuous-time phase retrieval

16.1. Continuous-time phase retrieval problem for structured signals with a full solution set of $2^{5}$ non-trivial ambiguities . . . . . . . . . . 169

VI. Phase retrieval from Fresnel magnitudes 
AMBIGUITIES IN

ONE-DIMENSIONAL PHASE

RETRIEVAL FROM FOURIER MAGNITUDES 



\section{Introduction}

If we only know the magnitude of a FOURIER transformed signal, can we recover the original signal, or exist there further signals with exactly the same FourIER intensity? In the unfortunate case that the recovery is ambiguous, how do the additional solutions look? Are they similar to the original signal, or can they have completely different shapes? How can we ensure that we always find the correct signal? These are the crucial questions, which we will investigate throughout this thesis.

More commonly, the problem to recover an unknown signal only from its FourIER intensity is called the phase retrieval problem since the phase of the signal in the frequency domain is lost. Recovery problems of this kind have many applications in physics and engineering. For example, the phase retrieval problem occurs in crystallography [Mil9o, Hau91, KH91], astronomy [DF87], and laser optics $\left[\mathrm{SSD}^{+} \mathrm{o6}\right]$. Finding an analytic or a numerical solution is generally challenging due to the well-known ambiguousness of the problem. In order to recover the original signal within the solution set, we hence require further a priori conditions or additional information about the wanted signal.

Since the phase retrieval problem is a current field of research for many decades, we can look back at a very rich literature about the analytical and numerical solvability of the phase retrieval problem for continuous-time and discrete-time signals with real and complex components in one and higher dimensions. Similarly, we find an extensive amount of different approaches to reduce the set of ambiguities and to recover the unknown signal uniquely. Nevertheless, the phase retrieval problem is not understood in full generality.

The goal of this thesis is twofold. On the one hand it surveys the existing literature about the ambiguities occurring in the phase retrieval problem with main emphases to the one-dimensional case. On the other hand, we develop a new complete characterization of all solutions in the one-dimensional discrete-time setting, which gives us new insights about the behavior of the problem. Based 
on our observations, we generalize the available results for real-valued signals to complex-valued signals, where the proofs can often be strongly simplified. Moreover, we extend the existing theory to reduce the solution set by novel findings. Afterwards, we transfer some of our contributions to the continuous-time setting and to the Fresnel case.

Let us briefly take a look at the rich literature about the ambiguousness of the phase retrieval problem with main focus to the one-dimensional case.

Continuous-time phase retrieval. Starting from a given FoURIER intensity $|\mathcal{F}[f]|$, in the one-dimensional continuous-time phase retrieval problem, one wants to find a complex-valued signal or a function $f$ whose FouRIER intensity coincides with the given data. In order to ensure that the Fourier intensity $\mathscr{F}[f]$ is well defined, the original signal $f$ has to be contained in a suitable function space. Assuming that the unknown signal $f$ is contained in $L^{1} \cap L^{2}$ and is supported on a half line, a first characterization of the occurring ambiguities of the problem has been given by using BLASCHKE products [Aku56, Aku57]. Under the slightly different assumption that the original signal $f$ is square-integrable and possesses a compact support, a further appropriate characterization of the complete solution set has been stated by employing the HADAMARD's factorization theorem for entire functions, see [Wal63, Hof64].

Since the continuous-time phase retrieval problem is not uniquely solvable in general, one has to state further appropriate a priori conditions on the unknown signal. One idea to achieve uniqueness of the problem is to assume that the unknown function is symmetric or monotone on its support [KST95]. Another approach to avoid ambiguities in the continuous-time phase retrieval is to extend the set of given Fourier intensities. For example, if the Fourier intensity of a modulation of the original signal with a complex exponential is available, one can solve the corresponding problem uniquely by comparing the zeros of the analytic continuation of the given intensities [WFB81]. Using a combination of oversampling and additional modulations, PoHL et al. have successfully recover complex signals with compact support [PYB14]. Moreover, the unknown signal can be uniquely reconstructed by exploiting interference measurements with a specially constructed reference signal depending on the given FourIER intensity [BFGR76].

Discrete-time phase retrieval. Although the phase retrieval problem was originally defined for the continuous-time setting, for numerical purposes, one needs to restrict the unknown continuous-time signal to a discrete space model. Therefore, we firstly restrict ourselves to the recovery of a discrete-time signal $x:=(x[n])_{n \in \mathbb{Z}}$ with finite support form its FourIER intensities $|\widehat{x}|$. Starting from the original signal $x$, here all further solutions of the considered phase retrieval 
problem can be constructed by considering the zeros of the $z$-transform of the autocorrelation signal, see [BS79, OS89]. Restricting the zeros used to construct the further solutions in a suitable way, one can easily ensure uniqueness of the phase retrieval problem as stated in [HLO8o].

In order to enforce uniqueness of the phase retrieval problem, sometimes additional information about signal values in the time domain are exploited. For example, if one of the endpoints of the wanted signal with finite support is completely known, most real-valued signals are completely determined, see [XYC87, SC91, Yag96, Yag98]. In [SSD ${ }^{+}$o6, LTo8, LTo9], a numerical algorithm to reconstruct an unknown complex-valued signal with finite support has been developed with the additional assumption that the absolute values of all signal components in time domain are available. Both approaches for complex-valued signals are more detailed investigated in Chapter III.

Being interested in additional information about the unknown signal for the phase retrieval problem that are physically available in the frequency domain, the idea of FOURIER intensities of suitable interferences has been extensively studied. Here, for real-valued signals, the interference with a known reference signal [KH9oa, $\mathrm{KH} 9 \mathrm{ob}$ ] can be employed to reduce the complete solution set to at most two different signals. Phase retrieval problems where the known reference is replaced by an unknown signal has been considered in [KH93] again for the real setting. Moreover, in [RDN13, $\mathrm{RSA}^{+}{ }_{11}$ ], this approach is generalized to the recovery of complex-valued signals. A special case of unknown interference signals is examined in [CESV13], where the reference is a modulated version of the original signal itself. We study the phase retrieval problem with additional interference measurements more closely in Chapter IV.

Another approach based on additional information in the frequency domain is the signal reconstruction from a signed FourIer intensity. This means that, beside the magnitude $|\widehat{x}|$ of the FOURIER transformed signal, the information whether $\widehat{x}(\omega)$ is contained in $[-\pi / 2, \pi / 2]$ or in $[-\pi,-\pi / 2) \cup(\pi / 2, \pi)$ is available. Exploiting this additional phase information, and fixing the finite support of the unknown real-valued signal $x$, one can show that this phase retrieval problem possesses a unique solution, see [HHLO83].

Similar results can be obtained by replacing the FouRIER transform by the so-called short-time FourIER transform [NQL83a, NQL83b], where the original signal is overlapped with a small analysis window at different positions. Choosing the analysis window appropriately, and supposing that the original signal satisfies some further conditions, one can recover this signal only from its shorttime FOURIER intensities.

If we assume that the finite support of the unknown signal $x$ is contained in the fixed set $\{0, \ldots, M-1\}$, we can consider this discrete-time signal as an $M$ - 
dimensional vector. Moreover, the FouRIER intensity $\left|\widehat{x}\left(\omega_{0}\right)\right|$ at a certain point $\omega_{0}$ can here be interpreted as the intensity measurement $|\langle x, v\rangle|$ with the vector $v:=\left(\mathrm{e}^{\mathrm{i} \omega_{0} m}\right)_{m=0}^{M-1}$. If one generalizes this approach to arbitrary frame vectors $v_{k}$, the questions arises how the frame vectors have to be constructed, and how many frame vectors are needed to ensure a unique recovery of the unknown vector $x$ from the given intensities $\left|\left\langle x, v_{k}\right\rangle\right|$. These issues have been extensively studied within the last years, see for instance [BCEo6, BBCEo9, ABFM14, BCM14, $\mathrm{BH}_{15}$ ] and references therein.

Multidimensional phase retrieval. Although we only consider the phase retrieval problem in one dimension, we want to make the point that the higherdimensional problem has a completely different behavior. Using a similar construction of the occurring ambiguities as in the one-dimensional real setting, one can observe that the ambiguities of the phase retrieval problem for higher dimensions depend on the factorization of a multivariate algebraic polynomial into irreducible polynomials, see [Hay82]. Since the reducible polynomials form a set of measure zero [HM82] in the space of all polynomials (up to a certain degree), almost every multidimensional signal can be uniquely recovered from its FourIER intensities.

Unfortunately, in some applications such as crystallography of certain objects [Milyo], the considered multidimensional signal always corresponds to a reducible polynomial, which means that the phase retrieval problem in this case cannot be uniquely solvable. In order to ensure the irreducibility, one can place a single reference point at a specific position outside the unknown object, see for instance [FBD83, DF84]. Other approaches are based on random illuminations [Fan12] or random masks [FL13]. Here the original multidimensional signal is only determined by the given intensities with high probability.

Fresnel phase retrieval. Physically, one can interpret the given Fourier magnitudes as intensity measurements of a wave on a plane in the far field. If we now replace the given Fourier intensity by measurements on a plane in the near field, then the new measurements correspond to the magnitudes of the FrESNEL transform, see [Goog6, LP12]. In other words, one is faced with the problem to recover an unknown signal from its Fresnel intensity. A further phase retrieval problem, which is closely related to the FrESNEL setting, is the recovery of a signal from its fractional FOURIER intensity, which corresponds to intensity measurements on a sphere in the near field, see [Nam8o, MK87, PF94]. Similarly to the FOURIER phase retrieval problem, the recovery of a signal from its FRESNEL or fractional FouRIER transform is again highly ambiguous. Assuming that one has access to intensity measurements of different distances, or that the unknown signal has a specific structure, it has been shown that both problems can nevertheless be solved uniquely [Jam14] . 
Outline. This work is organized as follows: Firstly, we consider the recovery of a one-dimensional discrete-time signal $x$ from its FourIER intensity $|\widehat{x}|$. Here we always assume that the unknown signal has a finite support. In other words, only finitely many components of the signal can be non-zero. This a priori condition can be interpreted as an additional sparsity constraint in a certain manner. More precisely, we wish to find the signal with the smallest support length to the given FOURIER intensities, where zero components within the signal support rarely occur. In order to get an impression about the ambiguities of the considered problem, we consider different special cases. Based on this observations, we distinguish between trivial and non-trivial ambiguities, cf. [Wan13].

The main statement of Chapter I is the complete mathematical classification of all solutions in the discrete-time phase retrieval. Instead of generalizing the results of BRUCK and SoDIN [BS79] for real-valued signals, we here use a completely different approach, where we examine the phase retrieval problem in the frequency domain, and employ a construction stated by FEJÉr and RiEsz [Fej16]. Together with the observation that the square of the given FoURIER intensity is nothing but a non-negative trigonometric polynomial, this allows us to show that there always exist at least one signal whose squared Fourier intensity coincides with an arbitrarily given non-negative trigonometric polynomial. In other words, the discrete-time phase retrieval problem always possesses at least one solution. Moreover, this novel approach yields an explicit product representation of all occurring ambiguities in the frequency domain. Transferring this observation to the time domain, we obtain the corresponding convolution representation. Finally, this enables us to conclude that all ambiguities can be described by rotations, shifts, reflection and conjugations, and appropriate convolutions.

Looking at the ambiguousness of the discrete-time phase retrieval problem, how can we ensure that we find the correct signal in the solution set? One a priori condition, which is exploited in many applications, is that the unknown signal is real-valued and non-negative. Unfortunately, the non-negativity condition cannot always achieve uniqueness as exemplarily shown in [Fie78, BS79]. In Chapter II, we consider the question: Are the given examples rare exceptions or the general case? Using our characterization, we will show that neither the signals that can be uniquely recovered nor the signals without a unique reconstruction form negligible sets. Consequently, the non-negativity is not suitable to ensure the uniqueness of the one-dimensional phase retrieval problem.

As mentioned above, XU, YAN, and CHANG [XYC87] exploited the exact end point of the otherwise unknown signal to show that almost all signals can be uniquely recovered. In Chapter III, we capture this idea and obtain a new approach to this specific recovery problem based on our characterization of all possible solutions. In this manner, we can generalize the original results for 
the real setting to complex-valued signals. Moreover, we give a series of novel proofs, which show that the known end point of the signal can be replaced by the assumption that the absolute value of one point or the phases of two points in the time domain are given beforehand.

In Chapter IV, we investigate the recovery of an unknown signal if the FourIER intensities of appropriate interferences are available. If the additional FouRIER intensities arise form the interference of the original signal with an also unknown reference, one can show that most signals are determined in the real setting by three FoURIER intensities, see [KH93]. In [RDN13], an analogous result is obtained for complex-valued signals by using four FouRIER intensities. Giving a new and complete proof for the complex version, we here show that most complex-valued signals are also determined by only three intensities. Inspired by [ABFM14, CESV13], we finally consider the interference with a known modulation of the signal itself. Adapting the existing results to our specific phase retrieval problem, and using Prony's method, we here additionally show that each signal can always be recovered uniquely up to a global phase.

Based on the phase retrieval of linear spline functions [SSD ${ }^{+}$o6, LTo8], we generalize this idea in Chapter $\mathrm{V}$ to the recovery of structured signals. Here most of our previous results for the discrete-time problem can be transferred straightforwardly. Using the characterization of the occurring ambiguities in the continuous-time setting [Hof64], we investigate the relation between the discrete-time and continuous-time problem. Moreover, we state some novel results about the recovery of an unknown continuous-time signal from interference measurements with an unknown reference signal or with a modulation of the unknown signal itself.

Finally, in Chapter VI, we consider the Fresnel phase retrieval problem. This means that we try to recover a discrete-time or continuous-time signal from the magnitudes of its Fresnel transform. Here we observe that the occurring ambiguities can be represented similarly to the solutions sets of the Fourier phase retrieval problem. This allows us to transfer the previous approches to ensure unique recovery in the FrESNEL setting.

Credits. Some of the findings presented in this thesis have been obtained in collaboration with Gerlind PlONKA-HocH and have been published in [BP15a, $\left.\mathrm{BP}_{15} \mathrm{~b}\right]$. In particular, the characterization of all possible ambiguities in the onedimensional discrete-time phase retrieval in Chapter I, the investigation of an additionally known end point of the signal in Chapter III, and the exploitation of interference measurements with a known and an unknown reference signal in Chapter IV have appeared in a slightly different form in the Fournal of Fourier Analysis and Applications [BP15a]. Moreover, the approach to reduce the 
continuous-time phase retrieval problem for structured function to a completely discrete setting have been published in PAMM, Proceedings in Applied Mathematics and Mechanics [BP15b]. 

CHAPTER I.

\title{
Characterization of the arising ambiguities
}

\begin{abstract}
We will begin our investigations about the ambiguities of the phase retrieval problem with the discrete-time setting. Here, for signals with real components, BRUCK and SoDIN [BS79] have successfully constructed all signals with a given FOURIER intensity up to a non-negative constant. Considering the phase retrieval problem in the frequency domain, and using a construction stated by FEJÉr and RIESZ [Fej16], we will here give a novel and complete proof to characterize all appearing ambiguities in the phase retrieval of a real-valued and furthermore complex-valued signal. Moreover, we will show that there always exist signals whose FOURIER intensity coincide with a given non-negative trigonometric polynomial. In other words, the discrete-time phase retrieval problem always possesses at least one solution. Retransforming our characterization to the time domain, we will conclude that all ambiguities can be described by rotations, shifts, reflection and conjugations, and appropriate convolutions. Finally, the complete characterization of all ambiguities will enables us to determine the number of non-trivially different solutions of a certain problem.
\end{abstract}

${ }^{a}$ Most of the observations in this chapter have been published in [BP15a].

\section{Formulation of the discrete-time problem}

Phase retrieval means that we wish to reconstruct an unknown signal from the intensity of its FourIER transform. Depending on whether the signal is discretetime or continuous-time, there are several ways to formulate the corresponding problem mathematically. Firstly, we examine the phase retrieval problem in the discrete-time setting. This means that we want to recover a discrete-time signal from its FourIER intensity.

Mathematically, a complex-valued discrete-time signal $x$ maps an integer $n$ to a complex number $x[n]$. Hence, the discrete-time signal $x$ is nothing but the 
complex-valued sequence

$$
x:=(x[n])_{n \in \mathbb{Z}} .
$$

Here we use the notation of [OS89, p. 9 et seq.]. In the following, we assume that the signal $x$ always has a finite support. In other words, only a finite number of components $x[n]$ are non-zero.

The FouriER transform of a discrete-time signal $x$ is defined for a real argument $\omega$ by the trigonometric series

$$
\mathscr{F}[x](\omega):=\widehat{x}(\omega):=\sum_{n \in \mathbb{Z}} x[n] \mathrm{e}^{-\mathrm{i} \omega n},
$$

see for instance [OS89, Section 2.7]. This specific transform is also called the discrete-time FOURIER transform. Obviously, the discrete-time FOURIER transform $\widehat{x}$ is a $2 \pi$-periodic function. Since we assume that the signal has a finite support, the series becomes a sum of finitely many exponentials and hence a complex trigonometric polynomial.

Example 1.1. As a first example, we consider the discrete-time signal

$$
\begin{aligned}
x:=\frac{1}{128}(\ldots, 0, \underline{55-15 i},-84+87 \mathrm{i}, 34+82 \mathrm{i}, \\
204-120 \mathrm{i},-16+16 \mathrm{i},-96,128,0, \ldots) .
\end{aligned}
$$

Here the underline marks the zeroth component $x[0]$ of the sequence. Since the support of the signal is $\{0, \ldots, 6\}$ and hence finite, the discrete-time FoURIER transform is the complex trigonometric polynomial $\widehat{x}$ given by

$$
\begin{aligned}
\widehat{x}(\omega)=\frac{1}{128} & \left((55+15 \mathrm{i})+(-84+87 \mathrm{i}) \mathrm{e}^{-\mathrm{i} \omega}+(34+82 \mathrm{i}) \mathrm{e}^{-2 \mathrm{i} \omega}\right. \\
+ & \left.(204-120 \mathrm{i}) \mathrm{e}^{-3 \mathrm{i} \omega}-(16-16 \mathrm{i}) \mathrm{e}^{-4 \mathrm{i} \omega}-96 \mathrm{e}^{-5 \mathrm{i} \omega}+128 \mathrm{e}^{-6 \mathrm{i} \omega}\right) .
\end{aligned}
$$

The considered signal $x$ and the FOURIER transform $\widehat{x}$ are graphically shown in Figure 1.1 on the facing page. Here the corresponding absolute value and phase of the complex-valued signal are presented in Figure 1.1(a) and (b). Moreover, in Figure 1.1(c), the signal $x$ is plotted as polygonal chain in the complex plane.

Analogously, in Figure 1.1(d), the $2 \pi$-periodic FourIER transform $\widehat{x}$ is plotted as a curve in the complex plane. The corresponding absolute value and phase are shown in Figure 1.1(e) and (f). The question behind the phase retrieval problem is: can we uniquely reconstruct the signal $x$ from the modulus of its Fourier transform in Figure 1.1(e) without the phase information in Figure 1.1(f)? 


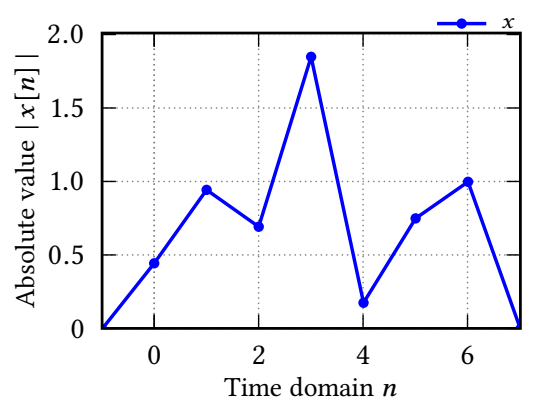

(a) Absolute value of the signal $x$ illustrated by a polygonal line

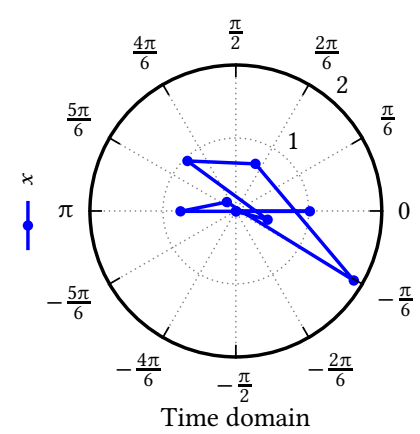

(c) Signal $x$ represented in polar form

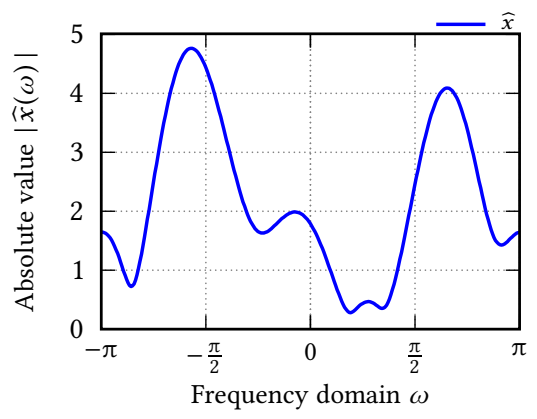

(e) Absolute value of the Fourier transform $\widehat{x}$

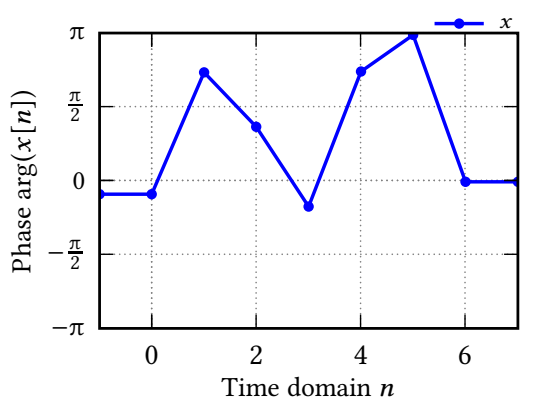

(b) Phase of the signal $x$ illustrated by a polygonal line

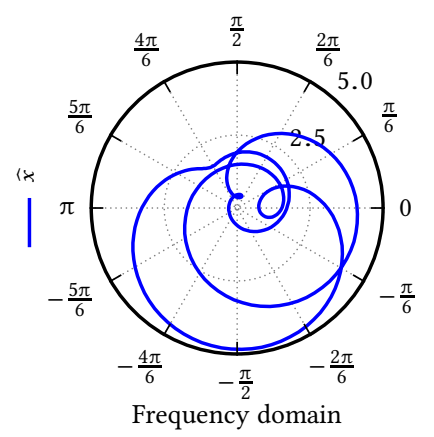

(d) FOURIER transform $\widehat{x}$ represented in polar form

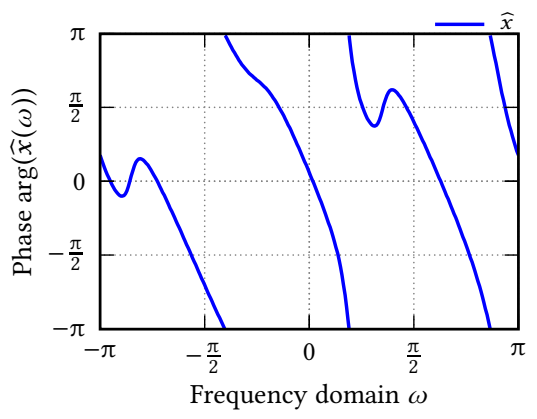

(f) Phase of the Fourier transform $\widehat{x}$

Figure 1.1.: Discrete-time signal with compact support and corresponding FourIER transform 
With the notations of the discrete-time signal and FOURIER transform, the onedimensional discrete-time phase retrieval problem can be written as follows.

Problem 1.2. The discrete-time phase retrieval problem is the problem of recovering a discrete-time signal $x$ with finite support from its FOURIER intensity $|\widehat{x}|$.

\section{Trivial and nontrivial ambiguities}

If we know the FOURIER transform $\widehat{x}$ of a discrete-time signal $x$ with finite support, then we can apply the inverse discrete-time FoURIER transform defined by

$$
x[n]=\frac{1}{2 \pi} \int_{-\pi}^{\pi} \widehat{x}(\omega) \mathrm{e}^{\mathrm{i} \omega n} \mathrm{~d} \omega,
$$

see for instance [OS89, Section 2.7], to recover the signal $x$ uniquely. However, knowing only the FourIER intensity, we have to recover the phase in the frequency domain in order to determine the signal itself uniquely.

Since the Fourier transform of a discrete-time signal with finite support is a trigonometric polynomial, the unknown phase in the frequency domain cannot be completely arbitrary. Nevertheless, the finite support cannot ensure a unique recovering of the signal. In order to formulate and examine suitable additional assumptions to enforce uniqueness of the phase retrieval problem, we are interested in the complete characterization of all occurring ambiguities.

Some of the simplest occurring ambiguities of the phase retrieval problem are the rotation (multiplication with an unimodular constant)

$$
\left(\mathrm{e}^{\mathrm{i} \alpha} x[n]\right)_{n \in \mathbb{Z}}
$$

for a some real $\alpha$, the time shift

$$
\left(x\left[n-n_{0}\right]\right)_{n \in \mathbb{Z}}
$$

for some integer $n_{0}$, and the reflection and conjugation

$$
(\overline{x[-n]})_{n \in \mathbb{Z}}
$$

of the original discrete-time signal $x$. 
Proposition 2.1. Let $x$ be a discrete-time signal with finite support and FOURIER intensity $|\widehat{x}|$. Then

(i) the rotated signal $\left(\mathrm{e}^{\mathrm{i} \alpha} x[n]\right)_{n \in \mathbb{Z}}$ for real $\alpha$

(ii) the time shifted signal $\left(x\left[n-n_{0}\right]\right)_{n \in \mathbb{Z}}$ for integer $n_{0}$

(iii) the reflected and conjugated signal $(\overline{x[-n]})_{n \in \mathbb{Z}}$

have the same FOURIER intensity $|\widehat{x}|$.

Proof. The assertion simply follows from the properties of the discrete-time FourIER transform, see for instance [OS89, Section 2.8 et seq.].

(i) Obviously, the FourIER transform of the rotated signal is given by

$$
\mathscr{F}\left[\left(\mathrm{e}^{\mathrm{i} \alpha} x[n]\right)_{n \in \mathbb{Z}}\right](\omega)=\mathrm{e}^{\mathrm{i} \alpha} \widehat{x}(\omega) .
$$

Since $\mathrm{e}^{\mathrm{i} \alpha}$ is a unimodular constant, the FOURIER intensity of the rotated signal is also $|\widehat{x}|$.

(ii) Using the definition of the FoURIER transform, and shifting the summation index by $n_{0}$, we have

$$
\begin{aligned}
\mathscr{F}\left[\left(x\left[n-n_{0}\right]\right)_{n \in \mathbb{Z}}\right](\omega) & =\sum_{n \in \mathbb{Z}} x\left[n-n_{0}\right] \mathrm{e}^{-\mathrm{i} \omega n} \\
& =\sum_{n \in \mathbb{Z}} x[n] \mathrm{e}^{-\mathrm{i} \omega\left(n+n_{0}\right)}=\mathrm{e}^{-\mathrm{i} \omega n_{0}} \widehat{x}(\omega) .
\end{aligned}
$$

Hence, the Fourier transform of the time shift differs from the Fourier transform of the original signal only by a unimodular factor.

(iii) Reversing the order of summation, and using the properties of the conjugation, the FOURIER transform of the reflected and conjugated signal is given by

$$
\mathscr{F}\left[(\overline{x[-n]})_{n \in \mathbb{Z}}\right](\omega)=\sum_{n \in \mathbb{Z}} \overline{x[-n]} \mathrm{e}^{-\mathrm{i} \omega n}=\overline{\sum_{n \in \mathbb{Z}} x[n] \mathrm{e}^{-\mathrm{i} \omega n}}=\overline{\widehat{x}(\omega)}
$$

so the FourIER transform is the conjugated Fourier transform of the original signal, and the FoURIER intensities of both signals are equal. 
Example 2.2. We consider again the phase retrieval problem to recover the discrete-time signal

$$
\begin{aligned}
x:=\frac{1}{128}(\ldots, 0, \underline{55-15 i},-84+87 \mathrm{i}, 34+82 \mathrm{i}, \\
204-120 \mathrm{i},-16+16 \mathrm{i},-96,128,0, \ldots)
\end{aligned}
$$

from its FOURIER intensity. Figure 2.1 on the next page shows the signal $x$; some ambiguities caused by rotation $x_{\mathrm{R}}$, time shift $x_{\mathrm{T}}$, and reflection and conjugation $x_{\mathrm{RC}}$; and the corresponding Fourier transforms. As shown in Proposition 2.1, the FourIER intensities of the signal and the ambiguities are equal.

For the ambiguity $x_{\mathrm{R}}$ caused by a rotation, we choose the real parameter $\alpha$ as $\pi / 2$. Hence, we have

$$
\begin{aligned}
x_{\mathrm{R}}:=\left(\mathrm{e}^{\mathrm{i} \frac{\pi}{2}} x[n]\right)_{n \in \mathbb{Z}}=\frac{1}{128}(\ldots, 0, \underline{15+55 \mathrm{i}},-87-84 \mathrm{i},-82+34 \mathrm{i}, \\
120+204 \mathrm{i},-16-16 \mathrm{i},-96 \mathrm{i}, 128 \mathrm{i}, 0, \ldots) .
\end{aligned}
$$

The multiplication with the unimodular factor i effects a phase shift in the time and frequency domain, see Figure 2.1(b) and (f), and a rotation around the origin in the complex plane, see Figure 2.1(c) and (d).

Next, we consider the ambiguity $x_{\mathrm{T}}$ caused by a time shift. As an example, we shift the signal $x$ to the left-hand side by setting the integer $n_{0}$ to -3 . This means that we define the signal $x_{\mathrm{T}}$ as

$$
\begin{aligned}
x_{\mathrm{T}}:=(x[n+3])_{n \in \mathbb{Z}}=\frac{1}{128}(\ldots, 0,55-15 \mathrm{i},-84+87 \mathrm{i}, 34+82 \mathrm{i}, \\
\underline{204-120 \mathrm{i}},-16+16 \mathrm{i},-96,128,0, \ldots) .
\end{aligned}
$$

The time shift of the signal causes a modulation of the phase in the frequency domain, see Figure 1.1(f).

Last, the reflection and conjugation $x_{\mathrm{RC}}$ of the signal $x$ is given by

$$
\begin{array}{r}
x_{\mathrm{RC}}:=(\overline{x[-n]})_{n \in \mathbb{Z}}=\frac{1}{128}(\ldots, 0,128,-96,-16-16 \mathrm{i}, 204+120 \mathrm{i} \\
34-82 \mathrm{i},-84-87 \mathrm{i}, \underline{55+15 \mathrm{i}}, 0, \ldots) .
\end{array}
$$

This ambiguity has the conjugated FourIER transform of the signal $x$, see again Figure 1.1(f).

Remark 2.3. In order to avoid the ambiguities caused by time shifts, the finite support of the considered signal $x$ can be normalized to the set $\{0, \ldots, N-1\}$, where $N$ is the length of the support. As an immediate consequence, the reflected 


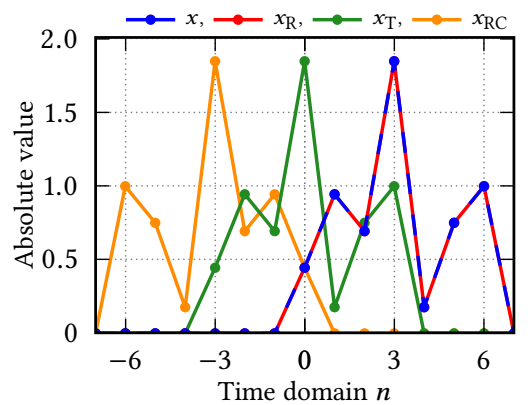

(a) Absolute value of the signals $x, x_{R}$, $x_{\mathrm{T}}$, and $x_{\mathrm{RC}}$ illustrated by polygonal lines

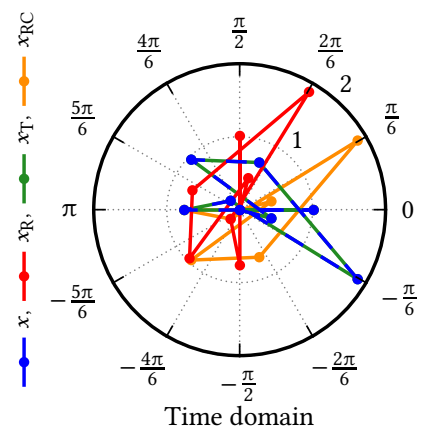

(c) Signals $x, x_{\mathrm{R}}, x_{\mathrm{T}}$, and $x_{\mathrm{RC}}$ represented in polar form

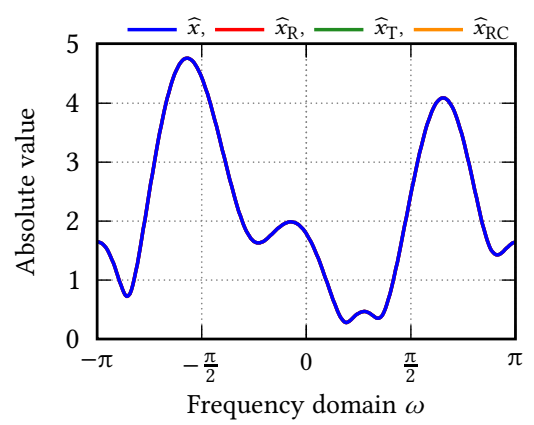

(e) Absolute value of the FouriER transforms $\widehat{x}, \widehat{x}_{\mathrm{R}}, \widehat{x}_{\mathrm{T}}$, and $\widehat{x}_{\mathrm{RC}}$

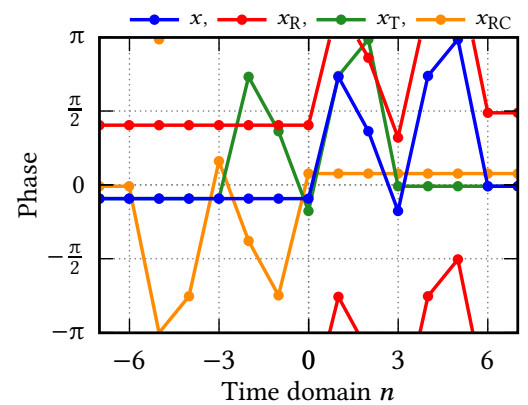

(b) Phase of the signals $x, x_{\mathrm{R}}, x_{\mathrm{T}}$, and $x_{\mathrm{RC}}$ illustrated by polygonal lines

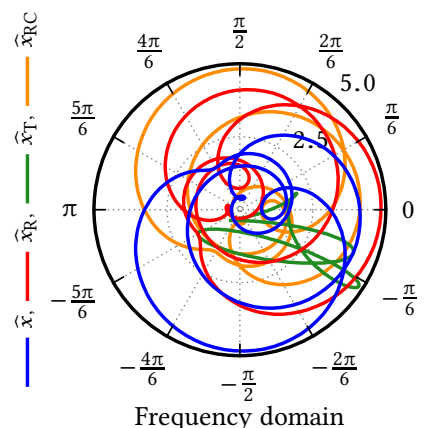

(d) FOURIER transforms $\widehat{x}, \widehat{x}_{\mathrm{R}}, \widehat{x}_{\mathrm{T}}$, and $\widehat{x}_{\mathrm{RC}}$ represented in polar form

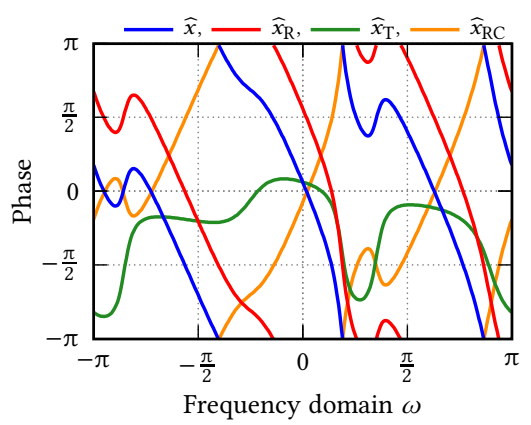

(f) Phase of the Fourier transforms $\widehat{x}$, $\widehat{x}_{\mathrm{R}}, \widehat{x}_{\mathrm{T}}$, and $\widehat{x}_{\mathrm{RC}}$

Figure 2.1.: Ambiguities caused by rotation, time shift, and reflection and conjugation in the discrete-time phase retrieval problem 
and conjugated signal possesses the reflected support $\{-N+1, \ldots, 0\}$ and has to be shifted by $N-1$ to restore the demanded support. If we take this additional shift into account, then the trivial ambiguity in Proposition 2.1(iii) caused by reflection and conjugation has the form

$$
(\overline{x[N-1-n]})_{n \in \mathbb{Z}} .
$$

As shown in Proposition 2.1, the rotation, time shift, or reflection and conjugation of a signal are ambiguities that always occur. But these three ambiguities are of minor interest since they are closely related to the original signal. However, we are mainly interested in the characterization of ambiguities which completely differ from the original signal and therefore distinguish between trivial and non-trivial ambiguities in analogy to [Wan13].

Definition 2.4. A trivial ambiguity of the discrete-time phase retrieval problem is caused by a rotation, time shift, or reflection and conjugation, or by a combinations of these. All other occurring ambiguities are called non-trivial.

Besides the trivial ambiguities caused by rotation, time shift, or reflection and conjugation, there is a further set of ambiguities which can be constructed in a simple manner. The description of these non-trivial ambiguities of the phase retrieval problem is based on the convolution of discrete-time signals, cf. [Wan13]. The convolution $x_{1} * x_{2}$ of two discrete-time signals $x_{1}$ and $x_{2}$ is defined by

$$
\left(x_{1} * x_{2}\right)[n]:=\sum_{k \in \mathbb{Z}} x_{1}[k] x_{2}[n-k]
$$

The Fourier transform of the convolution $x_{1} * x_{2}$ is given by the convolution theorem as

$$
\mathscr{F}\left[x_{1} * x_{2}\right]=\widehat{x}_{1} \cdot \widehat{x}_{2}
$$

see for instance [OS89, Section 2.9.6]. If we can factorize the original signal $x$ into two factors $x_{1}$ and $x_{2}$ with respect to the convolution, then we have the following ambiguities.

Proposition 2.5. Let $x$ be a discrete-time signal with finite support and FOURIER intensity $|\widehat{x}|$. Further, let $x_{1}$ and $x_{2}$ be two discrete-time signals with finite support such that

$$
x=x_{1} * x_{2} \text {. }
$$


Then, for real $\alpha$ and integer $n_{0}$, the signal

$$
\left(\mathrm{e}^{\mathrm{i} \alpha} \overline{x_{1}[-n]}\right)_{n \in \mathbb{Z}} *\left(x_{2}\left[n-n_{0}\right]\right)_{n \in \mathbb{Z}}
$$

has the same FOURIER intensity $|\widehat{x}|$.

Proof. The assertion follows directly by applying the convolution theorem, see for instance [OS89, Section 2.9.6], and Proposition 2.1. More detailed, we define the signal $y$ as

$$
y:=\left(\mathrm{e}^{\mathrm{i} \alpha} \overline{x_{1}[-n]}\right)_{n \in \mathbb{Z}} *\left(x_{2}\left[n-n_{0}\right]\right)_{n \in \mathbb{Z}} .
$$

Since the FOURIER transform of a convolution is the product of the FOURIER transformed factors, the convolution theorem of the discrete-time FouRIER transform yields

$$
\begin{aligned}
\widehat{y} & =\mathscr{F}\left[\left(\mathrm{e}^{\mathrm{i} \alpha} \overline{x_{1}[-n]}\right)_{n \in \mathbb{Z}} *\left(x_{2}\left[n-n_{0}\right]\right)_{n \in \mathbb{Z}}\right] \\
& =\mathscr{F}\left[\left(\mathrm{e}^{\mathrm{i} \alpha} \overline{x_{1}[-n]}\right)_{n \in \mathbb{Z}}\right] \cdot \mathscr{F}\left[\left(x_{2}\left[n-n_{0}\right]\right)_{n \in \mathbb{Z}}\right] .
\end{aligned}
$$

Now, taking the absolute value, and using that the occurring trivial ambiguities have the same FourIER intensity as the original signal (Proposition 2.1), we have

$$
\begin{aligned}
|\widehat{y}| & =\left|\mathcal{F}\left[\left(\mathrm{e}^{\mathrm{i} \alpha} \overline{x_{1}[-n]}\right)_{n \in \mathbb{Z}}\right]\right|\left|\mathcal{F}\left[\left(x_{2}\left[n-n_{0}\right]\right)_{n \in \mathbb{Z}}\right]\right| \\
& =\left|\widehat{x}_{1}\right|\left|\widehat{x}_{2}\right|=|\widehat{x}| .
\end{aligned}
$$

Hence, the signal $y$ and the original signal $x$ have the same FoURIER intensity, which finishes the proof.

Example 2.6. We continue the consideration of the phase retrieval problem in Example 2.2 to recover the signal

$$
\begin{aligned}
x:=\frac{1}{128}(\ldots, 0, \underline{55-15 i},-84+87 i, 34+82 \mathrm{i}, \\
204-120 \mathrm{i},-16+16 \mathrm{i},-96,128,0, \ldots) .
\end{aligned}
$$

from its FourIER intensity. Since the signal $x$ can be written as a convolution $x_{1} * x_{2}$ with the factors

$$
x_{1}:=\frac{1}{32}(\ldots, 0, \underline{7+4 i},-16,-8+16 \mathrm{i}, 32,0, \ldots)
$$




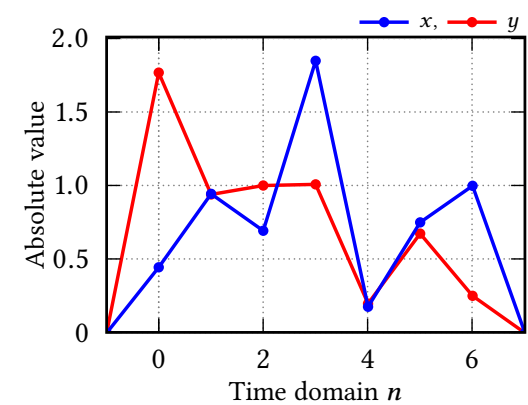

(a) Absolute value of the signals $x$ and $y$ illustrated by polygonal lines

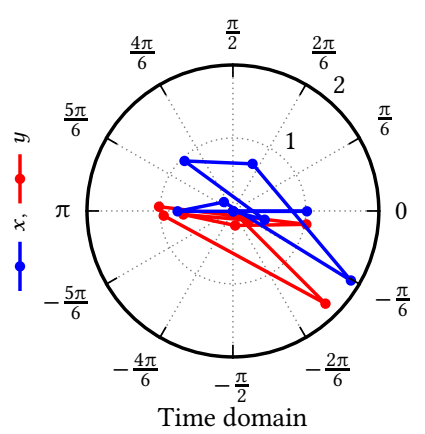

(c) Signals $x$ and $y$ represented in polar form

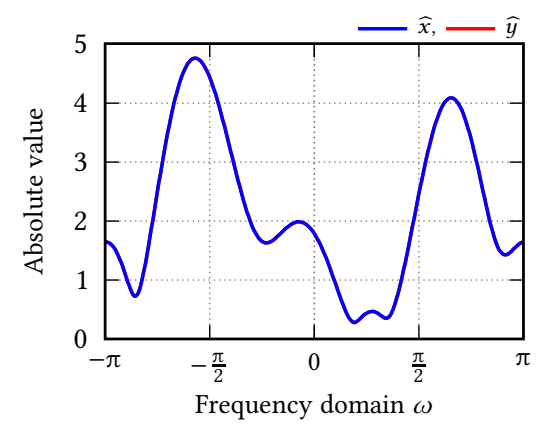

(e) Absolute value of the Fourier transforms $\widehat{x}$ and $\hat{y}$

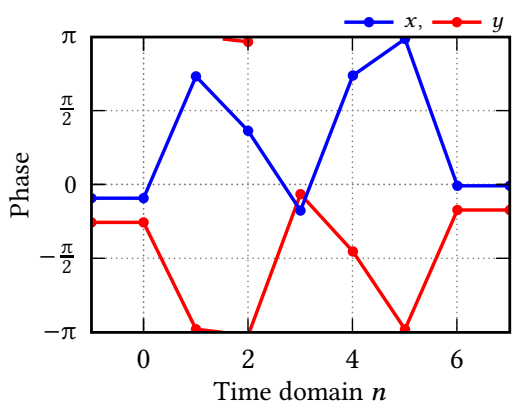

(b) Phase of the signals $x$ and $y$ illustrated by polygonal lines

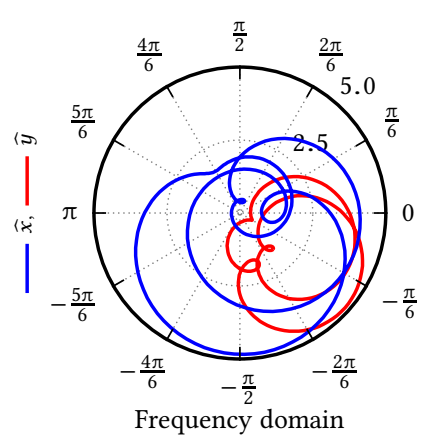

(d) FoURIER transforms $\widehat{x}$ and $\widehat{y}$ represented in polar form

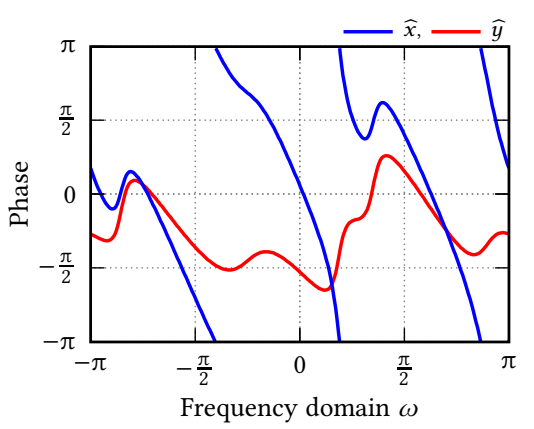

(f) Phase of the Fourier transforms $\hat{x}$ and $\widehat{y}$

Figure 2.2.: Non-trivial ambiguities of the discrete-time phase retrieval problem caused by convolution 
and

$$
x_{2}:=\frac{1}{4}(\ldots, 0, \underline{5-5 i}, \mathrm{i},-2-2 \mathrm{i}, 4,0, \ldots),
$$

we can apply Proposition 2.5 in order to construct a further ambiguity of the problem. More precisely, we consider the signal $y$ defined as

$$
y:=\left(\overline{x_{1}[-n]}\right)_{n \in \mathbb{Z}} *\left(x_{2}[n-3]\right)_{n \in \mathbb{Z}} .
$$

Here the first signal

$$
\left(\overline{x_{1}[-n]}\right)_{n \in \mathbb{Z}}=\frac{1}{32}(\ldots, 0,32,-8-16 \mathrm{i},-16, \underline{7-4 \mathrm{i}}, 0, \ldots) y
$$

is the reflection, conjugation, and 'rotation' by $\alpha=0$ of the signal $x_{1}$; and the second signal

$$
\left(x_{2}[n-3]\right)_{n \in \mathbb{Z}}=\frac{1}{4}(\ldots, 0, \underline{0}, 0,0,5-5 \mathrm{i}, \mathrm{i},-2-2 \mathrm{i}, 4,0, \ldots) .
$$

is the time shift of $x_{2}$ by $n_{0}=3$. Convolving both signals, we have

$$
\begin{aligned}
& y=\frac{1}{128}(\ldots, 0, 160-160 \mathrm{i},-120-8 \mathrm{i},-128+8 \mathrm{i}, \\
&127-23 \mathrm{i}, 4-25 \mathrm{i},-86-6 \mathrm{i}, 28-16 \mathrm{i}, 0, \ldots) .
\end{aligned}
$$

The original signal $x$ and the constructed signal $y$ are shown in Figure 2.2 on the facing page. Considering the absolute value of both signals in Figure 2.2(a), we can preclude that the ambiguity $y$ is caused by rotation, time shift, or reflection and conjugation. Hence, besides the trivial ambiguities, the considered phase retrieval problem possesses at least one non-trivial ambiguity.

Remark 2.7. Proposition 2.5 provides a procedure to find non-trivial ambiguities. But there may exist other non-trivial ambiguities that cannot be described as a convolution. However, by characterizing all occurring ambiguities of the discrete-time phase retrieval problem, we will see that every non-trivial ambiguity can indeed be described by a convolution as in Proposition 2.5. Hence, all ambiguities of the considered discrete-time phase retrieval problem can be characterized by using rotations, time shifts, reflections and conjugations, and convolutions.

Remark 2.8. Note that the constructed ambiguities in Proposition 2.5 do not always have to be non-trivial. For example, an ambiguity obtained by convolution 
is trivial if the first factor $x_{1}$ of the factorization $x=x_{1} * x_{2}$ is invariant under reflection and conjugation.

\section{The phase retrieval problem in the frequency domain}

To characterize all occurring ambiguities, we examine the phase retrieval problem in the frequency domain and derive an equivalent formulation where we have to find a root of a non-negative trigonometric polynomial. Our key instruments here are the autocorrelation signal and the autocorrelation function, see for instance [OS89, p. 65 et seq.].

Definition 3.1. Let $x$ be a discrete-time signal. The autocorrelation signal $a$ of the signal $x$ is defined by

$$
a[n]:=\sum_{k \in \mathbb{Z}} \overline{x[k]} x[k+n] .
$$

The autocorrelation function is the FOURIER transform $\widehat{a}$ of the autocorrelation signal $a$.

Remark 3.2. Since we only consider signals with finite support, the autocorrelation signal is always well-defined, and the support of the autocorrelation signal is finite. Hence, the autocorrelation function of a signal with finite support is a trigonometric polynomial.

Example 3.3. We consider the signal

$$
x:=(\ldots, \underline{0}, 0,3+\mathrm{i}, 5,1-2 \mathrm{i}, 0, \ldots) .
$$

Then the autocorrelation signal of $x$ is

$$
a=(\ldots, 0,1-7 \mathrm{i}, 20-15 \mathrm{i}, \underline{40}, 20+15 \mathrm{i}, 1+7 \mathrm{i}, 0, \ldots) .
$$

In this example, the autocorrelation signal is conjugate symmetric. This means that the components of $a$ fulfil the condition $\overline{a[-n]}=a[n]$ for all integer $n$. Furthermore, shifting the signal $x$ yields the same autocorrelation signal. Hence, the support of the autocorrelation signal only depends on the support length of the signal $x$ and is independent of the position of the support. 
The observations of Example 3.3 remain valid for all signals with finite support. Shifting the index of summation, the components of the autocorrelation signal fulfil the condition

$$
\overline{a[-n]}=\sum_{k \in \mathbb{Z}} x[k] \overline{x[k-n]}=\sum_{k \in \mathbb{Z}} x[k+n] \overline{x[k]}=a[n]
$$

for all integer $n$. Therefore, the autocorrelation signal is always conjugate symmetric. In particular, the zeroth component $a[0]$ of the autocorrelation signal has to be real.

Since the support length of a signal $x$ is defined as the largest integer $N$ such that there exist an integer $n_{0}$ with

$$
\overline{x\left[n_{0}\right]} x\left[n_{0}+N-1\right] \neq 0,
$$

the autocorrelation signal is obviously non-zero at the points $-N+1$ and $N-1$ and is zero for all integers $n$ with $|n| \geq N$. Hence, the support of autocorrelation signal is $\{-N+1, \ldots, N-1\}$ and only depends on the support length $N$ of the considered signal $x$.

Having defined the autocorrelation function, one may ask what is the relationship to the phase retrieval problem? The answer is given by the following proposition, see for instance [OS89, p. 65 et seq.].

Proposition 3.4. Let $x$ be a discrete-time signal with finite support. Then the squared FOURIER intensity $|\widehat{x}|^{2}$ is equal to the autocorrelation function $\widehat{a}$.

Proof. Writing the squared FourIEr intensity $|\widehat{x}|^{2}$ as the product $\widehat{x} \cdot \overline{\widehat{x}}$, and using the definition of the discrete-time FourIER transform, we have

$$
|\widehat{x}(\omega)|^{2}=\left(\sum_{n \in \mathbb{Z}} x[n] \mathrm{e}^{-\mathrm{i} \omega n}\right)\left(\sum_{k \in \mathbb{Z}} \overline{x[k]} \mathrm{e}^{\mathrm{i} \omega k}\right) .
$$

Since the signal $x$ has a finite support, all occurring sums are finite. Expanding the product and changing the order of summation now yields

$$
|\widehat{x}(\omega)|^{2}=\sum_{n \in \mathbb{Z}} \sum_{k \in \mathbb{Z}} x[n] \overline{x[k]} \mathrm{e}^{-\mathrm{i} \omega(n-k)}=\sum_{n \in \mathbb{Z}} \sum_{k \in \mathbb{Z}} \overline{x[k]} x[k+n] \mathrm{e}^{-\mathrm{i} \omega n} .
$$

With the definition of the autocorrelation signal and function, the assertion

follows.

$$
|\widehat{x}(\omega)|^{2}=\sum_{n \in \mathbb{Z}} a[n] \mathrm{e}^{-\mathrm{i} \omega n}=\widehat{a}(\omega)
$$


Remark 3.5. Assume that the support length $N$ of the signal $x$ is known. Since the squared FOURIER intensity $|\widehat{x}|$ is a $2 \pi$-periodic trigonometric polynomial of degree $N$, it is completely determined by $2 N-1$ samples at different points in the interval $[-\pi, \pi)$. If we have additional information about the support length, we can hence discretize the phase retrieval problem in the frequency domain too. An analogous observation follows if we only have an upper bound of the support length $N$. As a consequence, all results for the discrete-time phase retrieval problem (Problem 1.2) remain valid for a finite number of at least $2 N-1$ samples in the frequency domain.

The equality of the autocorrelation function and the squared FourIER intensity of a signal implies that the autocorrelation signal itself has to be a nonnegative trigonometric polynomial. Moreover, the degree of the autocorrelation function is $N-1$, where $N$ is again the support length of the considered signal, since the support of the autocorrelation signal is $\{-N+1, \ldots, N-1\}$ as seen above. Using the conjugate symmetry of the autocorrelation signal, we can write the autocorrelation function as

$$
\widehat{a}(\omega)=a[0]+\sum_{n=1}^{N-1}\left[a[n] \mathrm{e}^{-\mathrm{i} \omega n}+\overline{a[n]} \mathrm{e}^{\mathrm{i} \omega n}\right] .
$$

Further, splitting the components of the autocorrelation signal into real and imaginary parts gives

$$
\widehat{a}(\omega)=a[0]+\sum_{n=1}^{N-1} \mathfrak{R}(a[n])\left(\mathrm{e}^{-\mathrm{i} \omega n}+\mathrm{e}^{i \omega n}\right)+\sum_{n=1}^{N-1} \mathrm{i} \mathfrak{J}(a[n])\left(\mathrm{e}^{-\mathrm{i} \omega n}-\mathrm{e}^{\mathrm{i} \omega n}\right) .
$$

Applying EuLER's formula, we finally have

$$
\widehat{a}(\omega)=a[0]+2 \sum_{n=1}^{N-1} \mathfrak{R}(a[n]) \cos (\omega n)+2 \sum_{n=1}^{N-1} \mathfrak{J}(a[n]) \sin (\omega n),
$$

the real version of a trigonometric polynomial, see for instance [Kat68, p. 7].

On the other hand, an arbitrary non-negative trigonometric polynomial $T$ of degree $N-1$ defined by

$$
T(\omega):=\sum_{n=-N+1}^{N-1} c_{n} \mathrm{e}^{-\mathrm{i} \omega n}
$$

with complex coefficients $c_{n}$ is obviously invariant under complex conjugation. 
Reversing the order of summation in the definition of $\bar{T}$, this implies

$$
\overline{T(\omega)}=\sum_{n=-N+1}^{N-1} \bar{c}_{-n} \mathrm{e}^{-\mathrm{i} \omega n} \stackrel{!}{=} \sum_{n=-N+1}^{N-1} c_{n} \mathrm{e}^{-\mathrm{i} \omega n}=T(\omega) .
$$

Equating the coefficients of the trigonometric polynomials on both sides, we have $c_{n}=\bar{c}_{-n}$, see for instance [DM12, p. 94]. Hence, the coefficients of a nonnegative real trigonometric polynomial have to be conjugate symmetric like the autocorrelation signal.

In the original definition of the discrete-time phase retrieval problem (Problem 1.2), we aim to recover a signal from its FourIER intensity. If we consider the phase retrieval problem in the frequency domain, the problem is equivalent to reconstructing the symbol of a signal, the FourIER transformed signal, from a given autocorrelation function. Since the FOURIER transformed signal is a complex trigonometric polynomial, and since the autocorrelation function is a non-negative trigonometric polynomial, we are faced with the following problem, which is equivalent to Problem 1.2.

Problem 3.6. Let $T$ be a non-negative trigonometric polynomial. Find all complex trigonometric polynomials $R$ such that $|R|^{2}=T$.

In other words, we have to find a root $R$ of a trigonometric polynomial $T$. At the moment it is not clear that every non-negative trigonometric polynomial is the autocorrelation function of some signal. In particular, Problem 3.6 does not have to have a solution. However, if we can characterize all solutions of this problem, then we can use the inverse discrete-time FourIER transform to describe all ambiguities in the time domain.

Similarly as the phase retrieval problem in the time domain, Problem 3.6 is not uniquely solvable if at least one solution exist. If the trigonometric polynomial $R$ is a solution of $|R|^{2}=T$, then of course the rotation $\mathrm{e}^{\mathrm{i} \alpha} R$ for real $\alpha$, the modulation $\mathrm{e}^{-\mathrm{i} \omega n_{0}} R$ for integer $n_{0}$, and the conjugation $\bar{R}$ of the trigonometric polynomial $R$ are also solutions of the problem. In analogy to Definition 2.4, we call ambiguities caused by rotation, modulation, and conjugation trivial ambiguities. 


\section{Finding roots of a trigonometric polynomial}

We are faced with Problem 3.6 to find a root of a non-negative trigonometric polynomial. This problem is closely related to a question studied in [Fej16], where FEJÉr examines the issue whether all non-negative trigonometric polynomials of degree $N-1$ can be represented as the squared modulus of a complex algebraic polynomial of degree $N-1$ on the unit circle. FEJÉr and RIESZ show that this is always possible by constructing these algebraic polynomials explicitly.

Moreover, the construction in [Fej16] can be used to determine all complex trigonometric polynomials $R$ that fulfil the equation $|R|^{2}=T$ and hence solve Problem 3.6 for a given non-negative trigonometric polynomial $T$ of degree $N-1$ with

$$
T(\omega):=\sum_{n=-N+1}^{N-1} c_{n} \mathrm{e}^{-\mathrm{i} \omega n} .
$$

Following the way of FEJÉR and RIEsz, we define the associated polynomial $P$ for the trigonometric polynomial $T$ by

$$
P(z):=\sum_{n=0}^{2 N-2} c_{n-N+1} z^{n} .
$$

Hence, the associated polynomial is the algebraic polynomial of degree $2 \mathrm{~N}-2$ with the same coefficients as the trigonometric polynomial $T$.

Since we assume that the trigonometric polynomial $T$ is non-negative, the coefficients $c_{n}$ fulfil the condition $c_{n}=\bar{c}_{-n}$ as seen in Section 3 ; therefore, the associated polynomial $P$ is conjugate palindromic, which simply terms an algebraic polynomial whose coefficients are conjugate symmetric. Further, the associated polynomial $P$ is related to the trigonometric polynomial $T$ by

$$
T(\omega)=\mathrm{e}^{\mathrm{i} \omega(N-1)} P\left(\mathrm{e}^{-\mathrm{i} \omega}\right)
$$

and, in particular,

$$
T(\omega)=\left|P\left(\mathrm{e}^{-\mathrm{i} \omega}\right)\right|
$$

for real $\omega$; the absolute values of the trigonometric polynomial $T$ and the algebraic polynomial $P$ on the unit circle are equal.

\subsection{Examining the zero set of the associated polynomial}

The main idea of FEJÉr and RIESz in [Fej16] is to factorize the associated polynomial $P$ into linear factors and to rearrange these such that all linear factors occur 
twice under the absolute value. For this purpose, we have to determine the zeros with their multiplicity of the associated polynomial $P$.

Lemma 4.1. Let $T$ be a non-vanishing, non-negative trigonometric polynomial. If $\gamma$ is a zero of associated polynomial $P$ to $T$, then $\bar{\gamma}^{-1}$ is also a zero of $P$.

Proof. The associated polynomial $P$ to the trigonometric polynomial $T$ is a palindromic polynomial of degree $2 N-2$ as seen above; so the leading coefficient and the constant term are conjugated to each other and non-zero. As a consequence, we have $P(0) \neq 0$, and hence the considered zero $\gamma$ is non-zero. We follow the lines of FEJÉR and RIESz in [Fej16, p. 57] and consider the associated polynomial $P$ at the point $\bar{\gamma}^{-1}$. Reversing the order of summation in the definition of the associated polynomial, we have

$$
P\left(\bar{\gamma}^{-1}\right)=\sum_{n=0}^{2 N-2} c_{n-N+1} \bar{\gamma}^{-n}=\sum_{n=0}^{2 N-2} c_{N-1-n} \bar{\gamma}^{n-2 N+2} .
$$

Factoring out $\bar{\gamma}^{-2 N+2}$ and using the conjugate symmetry $c_{N-1-n}=\bar{c}_{n-N+1}$ of the coefficients yields

$$
P\left(\bar{\gamma}^{-1}\right)=\bar{\gamma}^{-2 N+2} \sum_{n=0}^{2 N-2} \bar{c}_{n-N+1} \bar{\gamma}^{n}=\bar{\gamma}^{-2 N+2} \overline{P(\gamma)}=0 .
$$

Since $\gamma$ is non-zero, the assertion follows.

Remark 4.2. Lemma 4.1 remains valid for an arbitrary conjugate palindromic polynomial and, moreover, for conjugate antipalindromic polynomials. A polynomial is called conjugate antipalindromic if the coefficients are conjugate antisymmetric. This means that the coefficients in definition (4.2) fulfil the condition $c_{n}=-\bar{c}_{-n}$. Here we obtain

$$
P\left(\bar{\gamma}^{-1}\right)=-\bar{\gamma}^{-2 N+2} \sum_{n=0}^{2 N-2} \bar{c}_{n-N+1} \bar{\gamma}^{n}=-\bar{\gamma}^{-2 N+2} \overline{P(\gamma)}=0
$$

instead of equation (4.5), and the statement follows in an analogous way.

Next, we will show that the zeros $\gamma$ and $\bar{\gamma}^{-1}$ of the associated polynomial $P$ in (4.2) occur with the same multiplicity. Since the derivatives of the associated polynomial are usually no longer conjugate palindromic, we cannot use 
Lemma 4.1 directly. In order to apply Lemma 4.1 also for the derivatives, we need to represent these in a suitable form. For this purpose, we define the polynomials $P^{[\ell]}$ for $\ell$ in $\mathbb{N}_{0}$ by

$$
P^{[\ell]}(z):=\sum_{n=0}^{2 N-2}(n-N+1)^{\ell} c_{n-N+1} z^{n}
$$

with the same coefficients as the associated polynomial $P$ in (4.2). As the associated polynomial itself, the polynomials $P^{[\ell]}$ are conjugate palindromic for even $\ell$ and conjugate antipalindromic for odd $\ell$. Further, the constructed polynomials are related by the following lemma.

Lemma 4.3. The algebraic polynomials in (4.6) fulfil the identity

$$
\frac{\mathrm{d}}{\mathrm{d} z} \frac{P^{[\ell]}(z)}{z^{N-1}}=\frac{P^{[\ell+1]}(z)}{z^{N}}
$$

for all $\ell$ in $\mathbb{N}_{0}$.

Proof. The statement simply follows by computing the derivative of the lefthand side. Multiplying the polynomial $P^{[\ell]}$ in (4.6) with $z^{-N+1}$, we have

$$
\frac{\mathrm{d}}{\mathrm{d} z} \frac{P^{[\ell]}(z)}{z^{N-1}}=\frac{\mathrm{d}}{\mathrm{d} z} \sum_{n=0}^{2 N-2}(n-N+1)^{\ell} c_{n-N+1} z^{n-N+1} .
$$

Taking the derivative of the polynomial, we can deduce the assertion by

$$
\frac{\mathrm{d}}{\mathrm{d} z} \frac{P^{[\ell]}(z)}{z^{N-1}}=\sum_{n=0}^{2 N-2}(n-N+1)^{\ell+1} c_{n-N+1} z^{n-N}=\frac{P^{[\ell+1]}(z)}{z^{N}}
$$

With the defined polynomials $P^{[\ell]}$ and Lemma $4 \cdot 3$, we can describe the derivatives of the associated polynomial $P$ as a sum of conjugate palindromic and conjugate antipalindromic polynomials.

Lemma 4.4. The $k$ th derivative of the associated polynomial $P$ in (4.2) to a trigonometric polynomial can be represented as

$$
P^{(k)}(z)=\sum_{\ell=0}^{k} \lambda_{\ell}^{(k)} \frac{P^{[\ell]}(z)}{z^{k}}
$$

with suitable coefficients $\lambda_{\ell}^{(k)}$. 
Proof. We prove the Lemma by mathematical induction over $k$. The assertion is obviously valid for $k=0$ since the associated polynomial $P$ and the polynomial $P^{[0]}$ coincide. In order to determine the $(k+1)$ st derivative of the associated polynomial, we use the induction hypothesis and extend the summands with $z^{N-1-k}$, which yields

$$
\frac{\mathrm{d}}{\mathrm{d} z} P^{(k)}(z)=\frac{\mathrm{d}}{\mathrm{d} z} \sum_{\ell=0}^{k} \lambda_{\ell}^{(k)} \frac{P^{[\ell]}(z)}{z^{k}}=\sum_{\ell=0}^{k} \lambda_{\ell}^{(k)} \frac{\mathrm{d}}{\mathrm{d} z}\left(z^{N-1-k} \frac{P^{[\ell]}(z)}{z^{N-1}}\right) .
$$

After applying the product rule together with Lemma 4.3, the $(k+1)$ st derivative can be written as

$$
P^{(k+1)}(z)=\sum_{\ell=0}^{k} \lambda_{\ell}^{(k)}\left[(N-1-k) \frac{P^{[\ell]}(z)}{z^{k+1}}+\frac{P^{[\ell+1]}(z)}{z^{k+1}}\right] .
$$

Finally, we define the coefficients $\lambda_{\ell}^{(k+1)}$ by

$$
\lambda_{\ell}^{(k+1)}:= \begin{cases}\lambda_{k}^{(k)} & \ell=k+1, \\ (N-1-k) \lambda_{\ell}^{(k)}+\lambda_{\ell-1}^{(k)} & \ell=1, \ldots, k, \\ (N-1-k) \lambda_{0}^{(k)} & \ell=0 .\end{cases}
$$

This finishes the proof.

Remark 4.5. The recursive definition of the coefficients $\lambda_{\ell}^{(k)}$ implies that $\lambda_{k}^{(k)}$ is always one. Hence, the polynomial $P^{[k]}$ always occurs in the representation of $P^{(k)}$ in Lemma $4 \cdot 4$.

In a further step, this specific form of the $k$ th derivative of the associated polynomial allows us to show that the zeros $\gamma$ and $\bar{\gamma}^{-1}$ of the associated polynomial occur with the same multiplicity.

Lemma 4.6. Let $T$ be a non-vanishing, non-negative trigonometric polynomial. If $\gamma$ is a zero of multiplicity $m$ of the associated polynomial to $T$, then $\bar{\gamma}^{-1}$ is also a zero of multiplicity $m$.

Proof. Applying Lemma 4.4 , we consider the $k$ th derivative of the associated polynomial $P$ defined in (4.2) at the point $\gamma$. Since $\gamma$ is a zero of multiplicity $m$, 
we have

$$
P^{(k)}(\gamma)=\sum_{\ell=0}^{k} \lambda_{\ell}^{(k)} \frac{P^{[\ell]}(\gamma)}{\gamma^{k}}=0
$$

for $k$ from 0 to $m-1$. For the reason that $\lambda_{k}^{(k)}$ is equal to one, as stated in Remark 4.5 , we inductively obtain that $\gamma$ is a zero of the algebraic polynomials $P^{[\ell]}$ for $\ell$ from 0 to $m-1$.

Moreover, together with Lemma 4.1 and Remark 4.2 , this implies that $\bar{\gamma}^{-1}$ is also a zero of $P^{[\ell]}$ for $\ell$ from 0 to $m-1$ because the polynomials $P^{[\ell]}$ are conjugate palindromic or conjugate antipalindromic. If we now evaluate the $k$ th derivative of the associated polynomial $P$ at the point $\bar{\gamma}^{-1}$, then we have

$$
P^{(k)}\left(\bar{\gamma}^{-1}\right)=\sum_{\ell=0}^{k} \lambda_{\ell}^{(k)} \frac{P^{[\ell]}\left(\bar{\gamma}^{-1}\right)}{\bar{\gamma}^{-k}}=0 .
$$

Hence, $\bar{\gamma}^{-1}$ is a zero of multiplicity at least $m$.

Repeating this observation for the $m$ th derivative, we see that both $\gamma$ and $\bar{\gamma}^{-1}$ cannot be a zero of $P^{[m]}$. As a consequence, the $m$ th derivative cannot vanish at the point $\bar{\gamma}^{-1}$. Consequently, $\gamma$ and $\bar{\gamma}^{-1}$ have to be of the same multiplicity $m$.

For a zero $\gamma$ on the unit circle, Lemma 4.6 is trivial since the reflection at the unit circle $\bar{\gamma}^{-1}$ is equal to the original point $\gamma$ itself. Nevertheless, in order to factorize the associated polynomial in an appropriate way, we also need that the zeros lying on the unit circle occur in 'pairs' of the form $\gamma$ and $\bar{\gamma}^{-1}$. In other words, we need to show that the zeros on the unit circle have even multiplicity.

Lemma 4.7. Let $T$ be a non-vanishing, non-negative trigonometric polynomial. The zeros of the associated polynomial $P$ in (4.2) to $T$ on the unit circle occur with even multiplicity.

Proof. Let $\gamma$ be a zero of the associated polynomial $P$ on the unit circle. This implies that we find a real $\omega_{0}$ such that $\gamma=\mathrm{e}^{-\mathrm{i} \omega_{0}}$. To show that $\gamma$ has even multiplicity, we will use the relationship (4.3) between the non-negative trigonometric polynomial $T$ and its associated polynomial $P$. Considering (4.3) for the point $\omega=\omega_{0}$, we have

$$
T\left(\omega_{0}\right)=\mathrm{e}^{\mathrm{i} \omega_{0}(N-1)} P\left(\mathrm{e}^{-\mathrm{i} \omega_{0}}\right)=0 .
$$


Hence, the zero $\gamma$ of the associated polynomial $P$ on the unit circle corresponds to the zero $\omega_{0}$ of the trigonometric polynomial $T$.

As the trigonometric polynomial $T$ is non-negative, the zero $\omega_{0}$ is a minimum of $T$ and hence has to be of even multiplicity $2 m$. In order to show that $\gamma=\mathrm{e}^{-\mathrm{i} \omega_{0}}$ is a zero of the associated polynomial with the same multiplicity $2 m$, we examine the complex derivatives and replace the real variable $\omega$ by the complex variable $z$ on the right-hand side of equation (4.3). By using the chain rule together with Lemma $4 \cdot 3$, the first complex derivative is given by

$$
\frac{\mathrm{d}}{\mathrm{d} z} \frac{P\left(\mathrm{e}^{-\mathrm{i} z}\right)}{\left(\mathrm{e}^{-\mathrm{i} z}\right)^{N-1}}=\frac{\mathrm{d}}{\mathrm{d} z} \frac{P^{[0]}\left(\mathrm{e}^{-\mathrm{i} z}\right)}{\left(\mathrm{e}^{-\mathrm{i} z}\right)^{N-1}}=-\mathrm{i} \frac{P^{[1]}\left(\mathrm{e}^{-\mathrm{i} z}\right)}{\left(\mathrm{e}^{-\mathrm{i} z}\right)^{N-1}} .
$$

Furthermore, the $k$ th complex derivative is inductively given by

$$
\frac{\mathrm{d}^{k}}{\mathrm{~d} z^{k}} \frac{P\left(\mathrm{e}^{-\mathrm{i} z}\right)}{\left(\mathrm{e}^{-\mathrm{i} z}\right)^{N-1}}=(-\mathrm{i})^{k} \frac{P^{[k]}\left(\mathrm{e}^{-\mathrm{i} z}\right)}{\left(\mathrm{e}^{-\mathrm{i} z}\right)^{N-1}} .
$$

Using that the trigonometric polynomial $T$ is real-valued, we can conclude that $\mathrm{e}^{\mathrm{i}(N-1) \cdot} P\left(\mathrm{e}^{-\mathrm{i} \cdot}\right)$ and all its derivatives $(4.8)$ are real-valued on the real axis. Since the remaining real parts of (4.8) for $z=\omega$ coincide with the derivatives of the trigonometric polynomial, the point $\gamma=\mathrm{e}^{-\mathrm{i} \omega_{0}}$ has to be a zero of the polynomials $P^{[\ell]}$ for $\ell$ from 0 to $2 m-1$. Moreover, the polynomial $P^{[2 m]}$ at the point $\gamma$ is non-zero. As we can represent the derivatives of the associated polynomial through the polynomials $P^{[\ell]}$, Lemma 4.4 implies that $\gamma$ is a zero of the first $2 m-1$ derivatives and no zero for the $2 m$ th derivative of $P$; therefore, $\gamma$ is a zero of even multiplicity $2 m$.

\subsection{Determining the trigonometric root polynomial}

With the preparatory work, Lemma 4.6 and Lemma 4.7 in particular, we can factorize the associated polynomial to a given non-negative trigonometric polynomial in a suitable way. Based on the work of FEJÉr and RIESZ in [Fej16], we can explicitly construct all complex trigonometric polynomials $R$ such that the squared modulus $|R|^{2}$ is equal to a given non-negative trigonometric polynomial. Thus, we can explicitly construct all solutions of Problem 3.6.

Theorem 4.8. Let $T$ be a non-vanishing, non-negative trigonometric polynomial of degree $N-1$. Each complex trigonometric polynomial $R$ satisfying $|R|^{2}=T$ can 
be written in the form

$$
R(\omega)=\mathrm{e}^{\mathrm{i}\left(\alpha-n_{0} \omega\right)} \sqrt{\left|c_{N-1}\right| \prod_{j=1}^{N-1}\left|\beta_{j}\right|^{-1}} \cdot \prod_{j=1}^{N-1}\left(\mathrm{e}^{-\mathrm{i} \omega}-\beta_{j}\right),
$$

where $\alpha$ is a real number, $n_{0}$ is an integer, and $\beta_{j}$ is chosen from the zero pair $\left(\gamma_{j}, \bar{\gamma}_{j}^{-1}\right)$ of the associated polynomial to $T$.

Proof. After the digression where we have examined the zero set of the associated polynomial, we return to the original construction of FEJÉr and RIESZ in [Fej16]. If $\gamma$ is a zero of the associated polynomial, then the reflection at the unit circle $\bar{\gamma}^{-1}$ is a further zero; and both zeros have the same multiplicity as seen in Lemma 4.6 and Lemma 4.7. For this reason, the zeros of the associated polynomial always occur in pairs of the form $\left(\gamma, \bar{\gamma}^{-1}\right)$. This means that we find a factorization of the associated polynomial $P$ to the given non-negative trigonometric polynomial $T$ in the form

$$
P(z)=c_{N-1} \prod_{j=1}^{N-1}\left(z-\gamma_{j}\right)\left(z-\bar{\gamma}_{j}^{-1}\right) .
$$

Using the relation between the trigonometric polynomial and the associated polynomial (4.4), we have

$$
T(\omega)=\left|c_{N-1}\right|\left|\prod_{j=1}^{N-1}\left(\mathrm{e}^{-\mathrm{i} \omega}-\gamma_{j}\right)\left(\mathrm{e}^{-\mathrm{i} \omega}-\bar{\gamma}_{j}^{-1}\right)\right| .
$$

Now, we rearrange the moduli of the factors on the right-hand side. By factoring out $\bar{\gamma}_{j}^{-1}$ in the second term, both linear factors are equal up to conjugation and sign. More precisely, we have the identity

$$
\left|\left(\mathrm{e}^{-\mathrm{i} \omega}-\gamma_{j}\right)\left(\mathrm{e}^{-\mathrm{i} \omega}-\bar{\gamma}_{j}^{-1}\right)\right|=\left|\mathrm{e}^{-\mathrm{i} \omega}-\gamma_{j}\right|\left|\bar{\gamma}_{j}^{-1}\right|\left|\bar{\gamma}_{j}-\mathrm{e}^{\mathrm{i} \omega}\right|=\left|\gamma_{j}\right|^{-1}\left|\mathrm{e}^{-\mathrm{i} \omega}-\gamma_{j}\right|^{2} .
$$

This implies that the given non-negative trigonometric polynomial $T$ can be written as

$$
T(\omega)=\left|c_{N-1}\right| \prod_{j=1}^{N-1}\left|\gamma_{j}\right|^{-1} \cdot\left|\prod_{j=1}^{N-1}\left(\mathrm{e}^{-\mathrm{i} \omega}-\gamma_{j}\right)\right|^{2} .
$$


Taking the trivial ambiguities obtained by rotation and modulation into account, we find the desired representation of $R$ given by

$$
R(\omega)=\mathrm{e}^{\mathrm{i}\left(\alpha-n_{0} \omega\right)} \sqrt{\left|c_{N-1}\right| \prod_{j=1}^{N-1}\left|\beta_{j}\right|^{-1}} \cdot \prod_{j=1}^{N-1}\left(\mathrm{e}^{-\mathrm{i} \omega}-\beta_{j}\right)
$$

where $\beta_{j}$ can be chosen from the zero pair $\left(\gamma_{j}, \bar{\gamma}_{j}^{-1}\right)$ of the associated polynomial.

It remains to prove that all complex trigonometric polynomials $R$ which satisfy the equation $|R|^{2}=T$ for a given non-negative trigonometric polynomial $T$ are of the stated form. Here we stop following the proof of FEJÉr and RIESZ since we do not assume that $R$ is of a certain degree as in [Fej16]. For this purpose, let $R$ given by $R(\omega):=\sum_{n \in \mathbb{Z}} b_{n} \mathrm{e}^{-\mathrm{i} \omega n}$ be a complex trigonometric polynomial such that $|R|^{2}=T$. Further, we define the support length $M$ of the coefficients sequence $\left(b_{n}\right)_{n \in \mathbb{Z}}$ in analogy to the support length of a signal. This means that $M$ is the largest integer such that there is an integer $m_{0}$ with $\bar{b}_{m_{0}} b_{m_{0}+M-1} \neq 0$. Note that $M$ and $m_{0}$ are uniquely defined since the coefficient sequence of a trigonometric polynomial has only finitely many non-zero components.

The squared modulus of the complex trigonometric polynomial $R$ is given by

$$
|R(\omega)|^{2}=R(\omega) \overline{R(\omega)}=\sum_{n \in \mathbb{Z}} \sum_{k \in \mathbb{Z}} b_{n} \bar{b}_{k} \mathrm{e}^{-\mathrm{i} \omega(n-k)}=\sum_{n \in \mathbb{Z}} \sum_{k \in \mathbb{Z}} b_{n+k} \bar{b}_{k} \mathrm{e}^{-\mathrm{i} \omega n},
$$

where all occurring sums are finite. Equating the coefficients of this trigonometric polynomial with the coefficients of the given non-negative trigonometric polynomial $T$ of degree $N-1$, we can conclude that the sums $\sum_{k \in \mathbb{Z}} b_{n+k} \bar{b}_{k}$ are zero for integer $n$ with $|n| \geq N$. This implies that the support length $M$ of the coefficients sequence $\left(b_{n}\right)_{n \in \mathbb{Z}}$ is at most $N$, otherwise

$$
\sum_{k \in \mathbb{Z}} b_{M-1+k} \bar{b}_{k}=b_{M-1+m_{0}} \bar{b}_{m_{0}} \neq 0
$$

with $M>N$ will contradict our observation that this sum has to be zero.

Moreover, the degree $N-1$ of the trigonometric polynomial $T$ implies that $\sum_{k \in \mathbb{Z}} b_{N-1+k} \bar{b}_{k}$ has to be non-zero. In other words, we find an integer $n_{0}$ such that the product $\bar{b}_{n_{0}} b_{N-1+n_{0}}$ is non-zero. As a consequence, the integer $M$ and $N$ must be equal, and $m_{0}$ and $n_{0}$ must coincide. Hence, by renumbering the coefficients $b_{n}$, the trigonometric polynomial $R$ has to be of the form

$$
R(\omega)=\mathrm{e}^{-\mathrm{i} n_{0} \omega} \sum_{n=0}^{N-1} b_{n} \mathrm{e}^{-\mathrm{i} \omega n}=\mathrm{e}^{-\mathrm{i} n_{0} \omega} b_{N-1} \prod_{j=1}^{N-1}\left(\mathrm{e}^{-\mathrm{i} \omega}-\beta_{j}\right)
$$


for a suitable integer $n_{0}$ and complex zeros $\beta_{j}$ in the factorization of the corresponding algebraic polynomial in $\mathrm{e}^{-\mathrm{i} \omega}$.

Next, we rearrange the modulus of the trigonometric polynomial $R$. Using the factorization of $R$ above, we have

$$
|R(\omega)|^{2}=\left|b_{N-1}\right|^{2} \prod_{j=1}^{N-1}\left(\mathrm{e}^{-\mathrm{i} \omega}-\beta_{j}\right)\left(\mathrm{e}^{\mathrm{i} \omega}-\bar{\beta}_{j}\right) .
$$

Factoring out $-\bar{\beta}_{j}$ and $\mathrm{e}^{\mathrm{i} \omega}$ in the second term in the product yields

$$
|R(\omega)|^{2}=\left|b_{N-1}\right|^{2} \mathrm{e}^{\mathrm{i} \omega(N-1)} \prod_{j=1}^{N-1}\left(-\bar{\beta}_{j}\right) \cdot \prod_{j=1}^{N-1}\left(\mathrm{e}^{-\mathrm{i} \omega}-\beta_{j}\right)\left(\mathrm{e}^{-\mathrm{i} \omega}-\bar{\beta}_{j}^{-1}\right) .
$$

Substituting $\mathrm{e}^{-\mathrm{i} \omega}=z$, we define the algebraic polynomial $Q$ by

$$
Q(z)=\left|b_{N-1}\right|^{2} \prod_{j=1}^{N-1}\left(-\bar{\beta}_{j}\right) \cdot \prod_{j=1}^{N-1}\left(z-\beta_{j}\right)\left(z-\bar{\beta}_{j}^{-1}\right)
$$

without the factor $\mathrm{e}^{\mathrm{i} \omega(N-1)}$.

The relation between the trigonometric and the associated polynomial (4.3) implies

$$
\left.\mathrm{e}^{\mathrm{i} \omega(N-1)} P\left(\mathrm{e}^{-\mathrm{i} \omega}\right)=T(\omega)=\mid R(\omega)\right)\left.\right|^{2}=\mathrm{e}^{\mathrm{i} \omega(N-1)} Q\left(\mathrm{e}^{-\mathrm{i} \omega}\right) .
$$

For this reason, the algebraic polynomials $P$ and $Q$ are equal on the unit circle and hence everywhere. In particular, the zero sets of both polynomials must coincide. After renumbering the zeros, this implies that the zero $\beta_{j}$ coincide with $\gamma_{j}$ or $\bar{\gamma}_{j}^{-1}$; therefore, the complex trigonometric polynomial $R$ has the stated form.

In the proof of Theorem 4.8, we have used an explicit construction for the roots of a given non-negative trigonometric polynomial. As a direct consequence, it follows that Problem 3.6, where we aim to characterize all trigonometric polynomials $R$ with $|R|^{2}=T$, has at least one solution for every non-negative trigonometric polynomial $T$.

Corollary 4.9. Let $T$ be a non-vanishing, non-negative trigonometric polynomial. There exist at least one complex trigonometric polynomial $R$ satisfying $|R|^{2}=T$. 
Remark 4.10. If we restrict the phase retrieval problem to the real case, where we want to recover a real-valued signal with compact support from its FourIER intensity, the components of the autocorrelation signal and the coefficient of the autocorrelation function in Definition 3.1 are real numbers. Using EuLER's formula and the (conjugate) symmetry $a[-n]=a[n]$ of the autocorrelation signal, we can write the autocorrelation function of a signal with support length $N$ in the form

$$
A(\omega)=a[0]+\sum_{n=1}^{N-1} a[n]\left(\mathrm{e}^{-\mathrm{i} \omega n}+\mathrm{e}^{\mathrm{i} \omega n}\right)=a[0]+2 \sum_{n=1}^{N-1} a[n] \cos (\omega n)
$$

Hence, the autocorrelation function is a non-negative even trigonometric polynomial.

In analogy to Problem 3.6, the corresponding problem in the frequency domain is to find all trigonometric polynomials $R$ with real coefficients such that $|R|^{2}=T$ for a given non-negative even trigonometric polynomial $T$. With an adapted version of the construction by FEJÉr and RIESz, it follows that there always exists at least one real trigonometric polynomial $R$ with $|R|^{2}=T$, see [Dau92, Lemma 6.1.3].

The same statement can be obtained by restricting the proof of Theorem 4.8 to the real case where the associated polynomial defined in (4.2) has only real coefficients; therefore, the real zeros of the associated polynomial occur in pairs

$$
\left(\gamma_{j}, \gamma_{j}^{-1}\right)
$$

and the complex zeros occur in quads

$$
\left(\gamma_{j}, \bar{\gamma}_{j}, \gamma_{j}^{-1}, \bar{\gamma}_{j}^{-1}\right)
$$

As in Theorem 4.8, all real solutions $R$ of the problem $|R|^{2}=T$ for a non-negative even trigonometric polynomial $T$ can be written in the form

$$
R(\omega)=\mathrm{e}^{\mathrm{i}\left(\alpha-n_{0} \omega\right)} \sqrt{\left|c_{N-1}\right| \prod_{j=1}^{N-1}\left|\beta_{j}\right|^{-1}} \cdot \prod_{j=1}^{N-1}\left(\mathrm{e}^{-\mathrm{i} \omega}-\beta_{j}\right)
$$

where the real $\beta_{j}$ can be chosen from the real zero pairs (4.9) and the complex $\beta_{j}$ as conjugate pairs from the complex zero quads (4.10).

Remark 4.11. Theorem 4.8 is closely related to observations done by BRUCK and SoDIN in [BS79], where the phase retrieval problem for non-negative signals with 
fixed support $\{0, \ldots, N-1\}$ is considered. Beginning with a given signal, BRUCK and SoDin construct all further solutions of the phase retrieval problem by using the $z$-transform of the given signal and the corresponding autocorrelation polynomial, the associated polynomial in (4.2). Investigating the problem in the frequency domain, we have moreover shown that each non-negative trigonometric polynomial is the autocorrelation function of at least one discrete-time signal, and that the corresponding phase retrieval problem consequently has at least one solution, see Corollary 4.9.

\section{Retransformation into the time domain}

Having a complete characterization of all occurring ambiguities in the frequency domain, we can apply the inverse discrete-time FouRIER transform (2.1) to characterize all ambiguities of the discrete-time phase retrieval problem (Problem 1.2) in the time domain. Consequently, we have to retransform the factorization of the trigonometric polynomial in Theorem 4.8. For this purpose, we consider the unit sample signal or the discrete-time impulse $\delta_{0}$ defined by

$$
\delta_{0}[n]:= \begin{cases}1 & n=0 \\ 0 & \text { else }\end{cases}
$$

The unit sample signal is the discrete-time analogue of the DIRAC $\delta$-distribution. Furthermore, we denote the shifted version of the unit sample signal by

$$
\delta_{n_{0}}:=\delta_{0}\left[\cdot-n_{0}\right]
$$

for an integer $n_{0}$.

The Fourier transform of the unit sample signal and its shifts is given by

$$
\widehat{\delta}_{n_{0}}(\omega)=\mathrm{e}^{-\mathrm{i} \omega n_{0}} .
$$

On the other side, the inverse Fourier transform of an exponential function $\mathrm{e}^{-\mathrm{i} n_{0} \cdot}$ for an integer $n_{0}$ is

$$
\mathscr{F}^{-1}\left[\mathrm{e}^{-\mathrm{i} n_{0} \cdot}\right]=\delta_{n_{0}}
$$


since we have

$$
\frac{1}{2 \pi} \int_{-\pi}^{\pi} \mathrm{e}^{\mathrm{i} \omega(n-m)} \mathrm{d} \omega= \begin{cases}1 & n=m \\ 0 & \text { else }\end{cases}
$$

for arbitrary integers $n$ and $m$, see for instance [Rud7o, p. 89]. Consequently, the inverse discrete-time FourIER transform of a trigonometric polynomial $T$ of degree $N-1$ with complex coefficients $c_{n}$ can be written as

$$
\mathscr{F}^{-1}[T]=\mathscr{F}^{-1}\left[\sum_{n=-N+1}^{N-1} c_{n} \mathrm{e}^{-\mathrm{i} n \cdot}\right]=\sum_{n=-N+1}^{N-1} c_{n} \delta_{n} .
$$

Here, the sum on the right-hand side is nothing but the coefficient sequence $\left(c_{n}\right)_{n \in \mathbb{Z}}$ with $c_{n}=0$ for integer $n$ with $|n| \geq N$.

Theorem 5.1. Let $x$ be a discrete-time signal with finite support and support length $N$. Each discrete-time signal y satisfying $|\widehat{y}|=|\widehat{x}|$ can be written in the form

$$
y=\mathrm{e}^{\mathrm{i} \alpha} \sqrt{|a[N-1]| \prod_{j=1}^{N-1}\left|\beta_{j}\right|^{-1}} \cdot\left[\delta_{n_{0}} * \underset{j=1}{\boldsymbol{*}}\left(\delta_{1}-\beta_{j} \delta_{0}\right)\right],
$$

where $\alpha$ is a real number, $n_{0}$ is an integer, $a$ is the autocorrelation signal to $x$, and $\beta_{j}$ is chosen from the zero pair $\left(\gamma_{j}, \bar{\gamma}_{j}^{-1}\right)$ of the associated polynomial to $|\widehat{x}|^{2}$.

Proof. The squared Fourier intensity $|\widehat{x}|^{2}$ is equal to the autocorrelation function $\widehat{a}$ of the signal $x$, see Proposition 3.4. For this reason, the corresponding problem in the frequency domain is to find all complex trigonometric polynomials $\widehat{y}$ with $|\widehat{y}|^{2}=\widehat{a}$. Due to the fact that the autocorrelation function $\widehat{a}$ is a non-negative trigonometric polynomial of degree $N-1$ as discussed in Section 3, we can apply Theorem 4.8 to characterize all solutions of the problem in the frequency domain. This means that $\widehat{y}$ can be written as

$$
\widehat{y}(\omega)=\mathrm{e}^{\mathrm{i}\left(\alpha-n_{0} \omega\right)} \sqrt{|a[N-1]| \prod_{j=1}^{N-1}\left|\beta_{j}\right|^{-1}} \cdot \prod_{j=1}^{N-1}\left(\mathrm{e}^{-\mathrm{i} \omega}-\beta_{j}\right),
$$

where $\alpha$ is a real number, $n_{0}$ is an integer, and $\beta_{j}$ is chosen from the zero pairs $\left(\gamma_{j}, \bar{\gamma}_{j}^{-1}\right)$ of the associated polynomial to $\widehat{a}=|\widehat{x}|^{2}$. 
Writing the inverse discrete-time FOURIER transform of a trigonometric polynomial as a sum of discrete-time impulses as in (5.1), we can write the retransformed 'linear factors' as

$$
\mathscr{F}^{-1}\left[\mathrm{e}^{-\mathrm{i} \omega}-\beta_{j} \mathrm{e}^{-\mathrm{i} \omega \cdot 0}\right]=\delta_{1}-\beta_{j} \delta_{0}
$$

Together with the convolution theorem (2.3), the inverse discrete-time FoURIER transform of $\widehat{y}$ yields the assertion.

In view of the factorization in Theorem 5.1, all solutions of the discrete-time phase retrieval problem for a given FouRIER intensity have exactly the same support length. This means that the Fourier intensity determines the length but not the exact position of the support.

Corollary 5.2. Let $x$ and $y$ be two discrete-time signals with finite support and the same FOURIER intensity $|\widehat{x}|$. Then both signals have the same support length.

Proof. Assuming that $x$ is a signal with support length $N$, we can write $y$ in the form

$$
y=\mathrm{e}^{\mathrm{i} \alpha} \sqrt{|a[N-1]| \prod_{j=1}^{N-1}\left|\beta_{j}\right|^{-1}} \cdot\left[\delta_{n_{0}} * \underset{j=1}{\boldsymbol{*}}\left(\delta_{1}-\beta_{j} \delta_{0}\right)\right]
$$

by Theorem 5.1. The commutativity, associativity, and distributivity of the discrete-time convolution implies that the convolution over the linear factors is of the form

$$
\underset{j=1}{*}\left(\delta_{1}-\beta_{j} \delta_{0}\right)=\sum_{n=0}^{N-1} c_{n} \delta_{n}
$$

with suitable complex coefficients $c_{n}$. In particular, the first and the last coefficient are given by

$$
c_{0}=\prod_{j=0}^{N-1}\left(-\beta_{j}\right) \quad \text { and } \quad c_{N-1}=1 .
$$

Due to the fact that the zeros $\beta_{j}$ in the factorization in Theorem 5.1 are non-zero, the coefficient $c_{0}$ is non-zero. Since the convolution with $\delta_{n_{0}}$ only causes a time shift by $n_{0}$, and since the remaining factors in the representation of $y$ are nonzero constants, the support length of the signals $y$ is also $N$, which completes the proof. 
The main finding of Theorem 5.1 is that each solution of the discrete-time phase retrieval problem can be written as a convolution of 'linear factors'. Further, up to rotations and shifts, each non-trivial ambiguity is completely characterized by the corresponding zero set $\left\{\beta_{j}: j=1, \ldots, N-1\right\}$ chosen from the zero pairs $\left(\gamma_{j}, \bar{\gamma}_{j}^{-1}\right)$ of the associated polynomial to the autocorrelation function.

Then again, we have seen that reflection and conjugation of individual factors in a factorization can cause non-trivial ambiguities, see Proposition 2.5 and Example 2.6. Moreover, using Theorem 5.1, we are going to prove that also the opposite is valid: all non-trivial ambiguities of the discrete-time phase retrieval problem can be described by reflecting and conjugating a factor in a suitable convolution. For this purpose, we will first examine the corresponding zero set of a reflected and conjugated signal.

Lemma 5.3. Let $x$ be a discrete-time signal with support length $N$ of the form

$$
x=C \cdot\left[\delta_{n_{0}} * \underset{j=1}{\mathbb{*}} \underset{\sim}{\boldsymbol{*}}\left(\delta_{1}-\beta_{j} \delta_{0}\right)\right]
$$

where $C$ is a complex number, $n_{0}$ is an integer, and $\beta_{j}$ are the corresponding non-zero zeros. Then the reflected and conjugated signal $\overline{x[-\cdot]}$ is of the form

$$
\overline{x[-\cdot]}=(-1)^{N-1} \bar{C} \prod_{j=1}^{N-1} \bar{\beta}_{j} \cdot\left[\delta_{-n_{0}-N+1} * \underset{j=1}{*} \underset{*}{*}\left(\delta_{1}-\bar{\beta}_{j}^{-1} \delta_{0}\right)\right] .
$$

Proof. In order to prove the statement of the lemma, we consider the factorization of $x$ in the frequency domain. Here, the Fourier transform of $x$ is given by

$$
\widehat{x}(\omega)=C \mathrm{e}^{-\mathrm{i} \omega n_{0}} \prod_{j=1}^{N-1}\left(\mathrm{e}^{-\mathrm{i} \omega}-\beta_{j}\right) .
$$

Due to the fact that the FouRIER transform of the reflected and conjugated signal is the conjugated FOURIER transform of the original signal, see for instance the proof of Proposition 2.1, we have

$$
\mathscr{F}[\overline{x[-\cdot]}](\omega)=\bar{C} \mathrm{e}^{\mathrm{i} \omega n_{0}} \prod_{j=1}^{N-1}\left(\mathrm{e}^{\mathrm{i} \omega}-\bar{\beta}_{j}\right)
$$


If we factor out $\mathrm{e}^{\mathrm{i} \omega}$ and $-\bar{\beta}_{j}$, we can write the linear factors in the product as

$$
\left(\mathrm{e}^{\mathrm{i} \omega}-\bar{\beta}_{j}\right)=\mathrm{e}^{\mathrm{i} \omega}\left(1-\bar{\beta}_{j} \mathrm{e}^{-\mathrm{i} \omega}\right)=-\bar{\beta}_{j} \mathrm{e}^{\mathrm{i} \omega}\left(\mathrm{e}^{-\mathrm{i} \omega}-\bar{\beta}_{j}^{-1}\right)
$$

and the FOURIER transform of the reflected and conjugated signal as

$$
\mathcal{F}[\overline{x[-\cdot]}](\omega)=\bar{C} \prod_{j=1}^{N-1}\left(-\bar{\beta}_{j}\right) \cdot \mathrm{e}^{\mathrm{i} \omega\left(n_{0}+N-1\right)} \prod_{j=1}^{N-1}\left(\mathrm{e}^{-\mathrm{i} \omega}-\bar{\beta}_{j}^{-1}\right) .
$$

Factoring out $(-1)^{N-1}$ and applying the inverse discrete-time FourIER transform yields the assertion.

Remark 5.4. The basic statement of Lemma 5.3 is that the reflection and conjugation of a signal in the time domain or the conjugation of the Fourier transformed signal in the frequency domain is characterized by reflecting all non-zero zeros $\beta_{j}$ in the factorization at the unit circle.

We are now ready to prove the inversion of Proposition 2.5 that all non-trivial ambiguities of the discrete-time phase retrieval problem can be represented by rotation, shift, reflection and conjugation, and convolution.

Theorem 5.5. Let $x$ and $y$ be two discrete-time signals with finite support and the same FOURIER intensity $|\widehat{x}|$. Then there exist two signals $x_{1}$ and $x_{2}$ such that

$$
x=x_{1} * x_{2}
$$

and

$$
y=\left(\mathrm{e}^{\mathrm{i} \alpha} \overline{x_{1}[-n]}\right)_{n \in \mathbb{Z}} *\left(x_{2}\left[n-n_{0}\right]\right)_{n \in \mathbb{Z}},
$$

where $\alpha$ is a suitable real number and $n_{0}$ is a suitable integer.

Proof. According to Theorem 5.1 both signals $x$ and $y$ are determined by the chosen zeros from the associated polynomial to the autocorrelation function up to an additional rotation and an additional shift. In order to simplify the notation, we assume that the signal $x$ corresponds to the zero set

$$
\left\{\beta_{j}: j=1, \ldots, N-1\right\}
$$


and the signal $y$ corresponds to

$$
\left\{\bar{\beta}_{j}^{-1}: j=1, \ldots, J\right\} \cup\left\{\beta_{j}: j=J+1, \ldots, N-1\right\}
$$

for some $J \in\{0, \ldots, N-1\}$, where one of the two sets in the union is empty for $J=0$ and $J=N-1$.

Moreover, without loss of generality, we assume that the factorization of $x$ does not contain an additional rotation and shift. In other words, we assume that the signal $x$ is of the form

$$
x=\sqrt{|a[N-1]| \prod_{j=1}^{N-1}\left|\beta_{j}\right|^{-1}} \cdot\left[\underset{j=1}{\mathbb{*}}\left(\delta_{1}-\beta_{j} \delta_{0}\right)\right],
$$

and that, for a real number $\phi$ and an integer $m_{0}$, the signal $y$ is of the form

$$
\begin{aligned}
y=\mathrm{e}^{\mathrm{i} \phi} \sqrt{|a[N-1]| \prod_{j=1}^{J}\left|\bar{\beta}_{j}\right| \cdot \prod_{j=J+1}^{N-1}\left|\beta_{j}\right|^{-1}} \\
\cdot\left[\delta_{m_{0}} * \underset{j=1}{\mathbb{*}}\left(\delta_{1}-\bar{\beta}_{j}^{-1} \delta_{0}\right) * \underset{j=J+1}{\mathbb{*}}\left(\delta_{1}-\beta_{j} \delta_{0}\right)\right],
\end{aligned}
$$

with the convention that

$$
\underset{j=1}{*}\left(\delta_{1}-\bar{\beta}_{j}^{-1} \delta_{0}\right)=\underset{j=N}{\stackrel{N-1}{*}}\left(\delta_{1}-\beta_{j} \delta_{0}\right)=\delta_{0} .
$$

Now, we define the first factor $x_{1}$ by

$$
x_{1}:=\sqrt{\prod_{j=1}^{J}\left|\beta_{j}\right|^{-1}} \cdot \underset{j=1}{\underset{*}{*}}\left(\delta_{1}-\beta_{j} \delta_{0}\right) .
$$

Hence, the signal $x_{1}$ corresponds to the zero set $\left\{\beta_{j}: j=1, \ldots, J\right\}$. Further, we define the second factor $x_{2}$ by

$$
x_{2}:=\sqrt{|a[N-1]| \prod_{j=J+1}^{N-1}\left|\beta_{j}\right|^{-1}} \cdot \underset{j=J+1}{\mathbb{*}}\left(\delta_{1}-\beta_{j} \delta_{0}\right)
$$

with the corresponding zero set $\left\{\beta_{j}: j=J+1, \ldots, N-1\right\}$. By choosing the factors $x_{1}$ and $x_{2}$ in this way, the signal $x$ is obviously the convolution of $x_{1}$ and $x_{2}$. 
According to Lemma 5.3, the conjugated and reflected signal $\overline{x_{1}[-\cdot]}$ corresponds to the reflected zero set. More precisely, we have the factorization

$$
\overline{x_{1}[-\cdot]}=\sqrt{|a[N-1]| \prod_{j=1}^{J}\left|\beta_{j}\right|^{-1}} \prod_{j=1}^{J}\left(-\bar{\beta}_{j}\right) \cdot\left[\delta_{-N+1} * \underset{j=1}{*} \underset{f}{*}\left(\delta_{1}-\bar{\beta}_{j}^{-1} \delta_{0}\right)\right] .
$$

Writing the zeros $\bar{\beta}_{j}$ in the prefactor as $\left|\bar{\beta}_{j}\right| \mathrm{e}^{-\mathrm{i} \arg \beta_{j}}$ yields the factorization

$$
\overline{x_{1}[-\cdot]}=\prod_{j=1}^{J}\left(-\mathrm{e}^{-\mathrm{i} \arg \beta_{j}}\right) \sqrt{|a[N-1]| \prod_{j=1}^{J}\left|\bar{\beta}_{j}\right|} \cdot\left[\delta_{-N+1} * \underset{j=1}{\boldsymbol{*}}\left(\delta_{1}-\bar{\beta}_{j}^{-1} \delta_{0}\right)\right] .
$$

Consequently, the second solution $y$ is, up to an additional rotation and shift, given by $\overline{x_{1}[-\cdot]} * x_{2}$. If we now choose $\alpha$ and $n_{0}$ in a suitable way, the proof is complete.

With Theorem 5.5, we have shown that all ambiguities, trivial and non-trivial, of the discrete-time phase retrieval problem can be described with the aid of rotations, shifts, reflections and conjugations, and convolutions. Moreover, the ambiguities considered in Proposition 2.1 and Proposition 2.5 are the only ambiguities that appear in the discrete-time phase retrieval problem. In particular, knowing one solution of a given phase retrieval problem, all other solutions can be constructed, at least theoretically, by Theorem 5.1 and Theorem 5.5.

\section{Number of non-trivial ambiguities}

One question which can be answered by Theorem 5.1 is: how many non-trivial ambiguities can occur in the discrete-time phase retrieval problem? Under the assumption that the unknown signal has the support $\{0, \ldots, N-1\}$ with support length $N$ and possesses only non-negative components, this issue is investigated by BRUCK and SoDIN in [BS79]. Considering that $\beta_{j}$ in Theorem 5.1 can be chosen from the zero pair $\left(\gamma_{j}, \bar{\gamma}_{j}^{-1}\right)$, we have, in analogy to [BS79, p. 305], a upper bound of $2^{N-1}$ not necessarily different signals that can be constructed by Theorem 5.1.

However, the maximal number of non-trivial ambiguities depends strongly on the zero set of the associated polynomial to the autocorrelation function. It is possible that nearly all ambiguities that can be constructed by Theorem 5.1 are non-trivial and distinct, but it is also possible that the phase retrieval problem is 
uniquely solvable. More precisely, the maximal number of non-trivial ambiguities that can be constructed by Theorem 5.1 depends on the multiplicity of the zero pairs of the associated polynomial.

Proposition 6.1. Let $x$ be a discrete-time signal with finite support. Furthermore, let $L$ be the number of distinct zero pairs $\left(\gamma_{\ell}, \bar{\gamma}_{\ell}^{-1}\right)$ of the associated polynomial to the autocorrelation function not lying on the unit circle, and let $m_{\ell}$ be the multiplicity of these zero pairs. The corresponding discrete-time phase retrieval problem to recover the signal $x$ has

$$
\left\lceil\frac{1}{2} \prod_{\ell=1}^{L}\left(m_{\ell}+1\right)\right\rceil
$$

non-trivial ambiguities.

Proof. In order to determine the exact number of non-trivial ambiguities of a specific phase retrieval problem, we examine the characterization

$$
\mathrm{e}^{\mathrm{i} \alpha} \sqrt{|a[N-1]| \prod_{j=1}^{N-1}\left|\beta_{j}\right|^{-1}} \cdot\left[\delta_{n_{0}} * \underset{j=1}{\boldsymbol{*}}\left(\delta_{1}-\beta_{j} \delta_{0}\right)\right]
$$

of all possible solutions in Theorem 5.1. Since the real number $\alpha$ and the integer $n_{0}$ merely describe rotations and shifts respectively, the non-trivial ambiguities only depend on the chosen zero sets $\left\{\beta_{j}: j=1, \ldots, N-1\right\}$. To prove the assertion, we will first answer the question: how many different zero sets can occur depending on the position and the multiplicity of the distinct zero pairs $\left(\gamma_{\ell}, \bar{\gamma}_{\ell}^{-1}\right)$ of the associated polynomial to the autocorrelation function?

In this consideration, we can neglect the zero pairs lying on the unit circle since both zeros coincide in this case, and hence the corresponding zeros $\beta_{j}$ in the factorization of the solution are fixed. For a zero pair $\left(\gamma_{\ell}, \bar{\gamma}_{\ell}^{-1}\right)$ with multiplicity $m_{\ell}$ not lying on the unit circle, we have $m_{\ell}$ corresponding zeros $\beta_{j}$ chosen from $\left(\gamma_{\ell}, \bar{\gamma}_{\ell}^{-1}\right)$ in the factorization. Due to the fact that we can pick up to $m_{\ell}$ zeros $\beta_{j}$ inside the unit circle and the remaining zeros $\beta_{j}$ outside, we have $m_{\ell}+1$ different possibilities to choose the corresponding zeros $\beta_{j}$ for the zero pair $\left(\gamma_{\ell}, \bar{\gamma}_{\ell}^{-1}\right)$. So all in all, there exactly occur

$$
\prod_{\ell=1}^{L}\left(m_{\ell}+1\right)
$$

different zero sets $\left\{\beta_{j}: j=1, \ldots, N-1\right\}$ for the characterization in Theorem 5.1, cf. [Fej16, p. 61]. 
However, the signals constructed by Theorem 5.1 do not have to be non-trivially different. Since a signal is completely determined by the corresponding zeros set $\left\{\beta_{j}: j=1, \ldots, N-1\right\}$ except for a multiplicative constant - especially a rotation - and an additional shift, we can exclude that the signals characterized in Theorem 5.1 are rotated or shifted versions of each other for different zero sets. Hence, trivial ambiguities can only arise together with a reflection and conjugation.

In Lemma 5.3, we have seen that the reflected and conjugated signal corresponds to the zero set reflected at the unit circle. Consequently, all signals constructed by Theorem 5.1 for different zero sets can be paired such that one signal can be obtained up to a rotation or a shift by reflecting and conjugating the other signal of the pair. The only exception is the signal that is invariant under reflection and conjugation, and that hence cannot be paired with a second signal. Since the corresponding zero set of such a signal has to be invariant under reflection at the unit circle, we can construct at most one invariant signal. Considering these remaining trivial ambiguities, we finally obtain the claimed number of non-trivial ambiguities.

Remark 6.2. If we restrict the discrete-time phase retrieval problem to the real case (see Remark 4.10), then the real zeros $\beta_{j}$ in the characterization of all arising ambiguities (Theorem 5.1) can be chosen from the real pair

$$
\left(\gamma_{j}, \gamma_{j}^{-1}\right)
$$

The complex zeros, however, must be chosen as conjugate pairs $\left(\beta_{j}, \bar{\beta}_{j}\right)$ from the quads

$$
\left(\gamma_{j}, \bar{\gamma}_{j}, \gamma_{j}^{-1}, \bar{\gamma}_{j}^{-1}\right)
$$

Therefore, it is sufficient to consider only the multiplicity of zero pairs $\left(\gamma_{j}, \bar{\gamma}_{j}^{-1}\right)$ in the upper half plane to determine the number of occurring zero sets of realvalued signal in (6.1), cf. [Fej16, p. 61]. This observation can now be transferred to maximal number of possible non-trivial ambiguities in Proposition 6.1 without circumstances.

Corollary 6.3. Let $x$ be a discrete-time signal with support length $N$. The number of non-trivial ambiguities of the corresponding discrete-time phase retrieval problem to recover the signal $x$ may vary from 1 up to $2^{N-2}$. 
Proof. If we consider a zero $\gamma_{j}$ of the associated polynomial to the autocorrelation function with modulus one, then the zero pair $\left(\gamma_{j}, \bar{\gamma}_{j}^{-1}\right)$ is reduced to one two-fold zero since both values coincide. Consequently, the zeros $\beta_{j}$ chosen from the pair $\left(\gamma_{j}, \bar{\gamma}_{j}^{-1}\right)$ are uniquely determined whenever all zeros of the associated polynomial lie on the unit circle. In this specific case, all zero sets $\left\{\beta_{j}: j=1, \ldots, N-1\right\}$ of the characterization in Theorem 5.1 coincide, and the corresponding phase retrieval problem is uniquely solvable up to rotations and shifts.

As seen in Proposition 6.1, the number of non-trivial solutions of a phase retrieval problem depends on the number of distinct zero pairs $\left(\gamma_{\ell}, \bar{\gamma}_{\ell}^{-1}\right)$ of the associated polynomial not lying on the unit circle and their multiplicities $m_{\ell}$. With the inequality $m_{\ell}+1 \leq 2^{m_{\ell}}$, and since the multiplicities of all occurring zero pairs $\left(\gamma_{j}, \bar{\gamma}_{j}^{-1}\right)$ sum up to $N-1$, we can estimate the number of non-trivial ambiguities in Proposition 6.1 by

$$
\left\lceil\frac{1}{2} \prod_{\ell=1}^{L}\left(m_{\ell}+1\right)\right\rceil \leq\left\lceil\frac{1}{2} \prod_{\ell=1}^{L} 2^{m_{\ell}}\right\rceil \leq 2^{N-2} .
$$

Furthermore, Proposition 6.1 implies that the largest number $2^{N-2}$ of non-trivial ambiguities is attained in the case where no zero pair lies on the unit circle, and where all zero pairs occur with multiplicity one.

Remark 6.4. Similarly as in Corollary 6.3 above, the discrete-time phase retrieval problem to recover a real signal can have up to $2^{N-2}$ non-trivial ambiguities. In this specific case, the upper bound $2^{N-2}$ can only be attained when all zeros of the associated polynomial to the autocorrelation function are real. On the contrary, if all occurring zeros are complex and hence arise in conjugate pairs, then we have at most $2^{\frac{N-1}{2}-1}$ non-trivially different solutions.

If we look back at the number of non-trivially different solutions in Proposition 6.1, we can find phase retrieval problems with a certain number of nontrivial ambiguities between 1 and $2^{N-2}$. Therefore, we want to give a series of examples which illustrate some specific cases - from a unique solution to the full solution set with $2^{N-2}$ non-trivial ambiguities.

Example 6.5. The phase retrieval problem in the proof of Corollary 6.3, where all zeros of the associated polynomial lie on the unit circle, is merely an example for a uniquely solvable problem. More precisely, a discrete-time signal can be recovered up to trivial ambiguities if all but at most one corresponding zero lie 


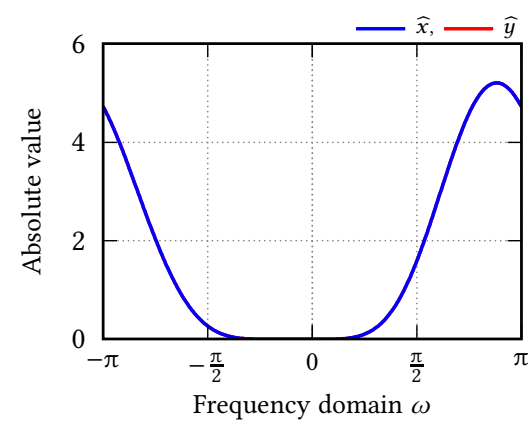

(a) Fourier intensities of the signals $x$ and $y$

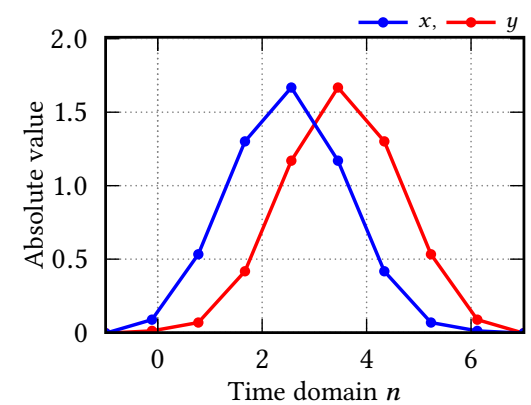

(c) Absolute value of the signals $x$ and $y$ illustrated by polygonal lines

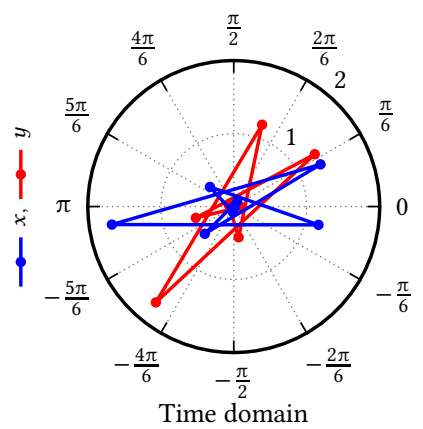

(b) Signals $x$ and $y$ represented in polar form

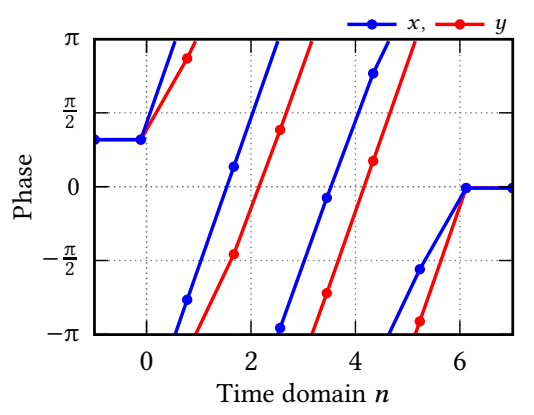

(d) Phase of the signals $x$ and $y$ illustrated by polygonal lines

Figure 6.1.: Discrete-time phase retrieval problem with a unique solution up to trivial ambiguities

on the unit circle. This directly follows from Proposition 6.1 with $L=1$ and $m_{1}=1$ since only one simple zero pair not lying on the unit circle appears.

As a specific example, we try to recover the signal

$$
x:=\frac{1}{64}\left(\delta_{1}+(5-3 \mathrm{i}) \delta_{0}\right) * \underset{j=1}{\underset{6}{6}}\left(\delta_{1}-\mathrm{e}^{\mathrm{i} \frac{\pi}{12}} \delta_{0}\right)
$$

from its FOURIER intensity $|\widehat{x}|$ given by

$$
|\widehat{x}(\omega)|^{2}=\frac{1}{64^{2}}\left|\left(\mathrm{e}^{-\mathrm{i} \omega}+5-3 \mathrm{i}\right)\left(\mathrm{e}^{-\mathrm{i} \omega}+\frac{1}{5+3 \mathrm{i}}\right)\right|\left|\mathrm{e}^{-\mathrm{i} \omega}-\mathrm{e}^{\mathrm{i} \frac{\pi}{12}}\right|^{12} .
$$

Since all but one corresponding zeros of the signal $x$ lie on the unit circle and are completely determined by the autocorrelation function, we can only construct 
one other solution

$$
y:=\frac{\sqrt{34}}{64}\left(\delta_{1}+\frac{1}{5+3 \mathrm{i}} \delta_{0}\right) * \underset{j=1}{\underset{*}{*}}\left(\delta_{1}-\mathrm{e}^{\mathrm{i} \frac{\pi}{12}} \delta_{0}\right)
$$

by Theorem 5.1. The corresponding zero set of this signal is, however, the zero set of the original signal $x$ reflected at the unit circle; so the signals $x$ and $y$ are reflected and conjugated versions of each other, apart from an additional rotation, see Lemma 5.3 and Figure 6.1 on the preceding page. Hence, we can recover $x$ uniquely up to trivial ambiguities.

Example 6.6. Although we can construct $2^{N-1}$ signals by using the characterization of all ambiguities in Theorem 5.1 and choosing $\beta_{j}$ as $\gamma_{j}$ or $\bar{\gamma}_{j}^{-1}$, the number of non-trivially different solutions does not have to be a power of two. As seen in Proposition 6.1, we can simply construct such phase retrieval problems by selecting corresponding zeros with an appropriate multiplicity.

For instance, the discrete-time phase retrieval problem with the FourIER intensity $|\widehat{x}|$ defined by

$$
|\widehat{x}(\omega)|^{2}=\left|\left(\mathrm{e}^{-\mathrm{i} \omega}+\frac{1}{2}\right)\left(\mathrm{e}^{-\mathrm{i} \omega}+2\right)\right|^{4} \cdot\left|\mathrm{e}^{-\mathrm{i} \omega}+\mathrm{e}^{\mathrm{i} \frac{\pi}{10}}\right|^{10}
$$

has exactly three non-trivially different solutions, namely

$$
\begin{gathered}
x_{1}=\underset{j=1}{\underset{*}{*}}\left(\delta_{1}+\frac{1}{2} \delta_{0}\right) * \underset{j=1}{\underset{*}{*}}\left(\delta_{1}+\mathrm{e}^{\mathrm{i} \frac{\pi}{10}} \delta_{0}\right), \\
x_{2}=\frac{1}{2} \underset{j=1}{\stackrel{*}{*}}\left(\delta_{1}+\frac{1}{2} \delta_{0}\right) *\left(\delta_{1}+2 \delta_{0}\right) * \underset{j=1}{\underset{*}{*}}\left(\delta_{1}+\mathrm{e}^{\mathrm{i} \frac{\pi}{10}} \delta_{0}\right),
\end{gathered}
$$

and

$$
x_{3}=\frac{1}{4} \underset{j=1}{\stackrel{*}{*}}\left(\delta_{1}+\frac{1}{2} \delta_{0}\right) * \underset{j=1}{\stackrel{*}{*}}\left(\delta_{1}+2 \delta_{0}\right) * \underset{j=1}{\underset{*}{*}}\left(\delta_{1}+\mathrm{e}^{\mathrm{i} \frac{\pi}{10}} \delta_{0}\right) .
$$

Switching more than two zeros $1 / 2$ outside the unit circle to 2 only produces further trivial ambiguities caused by reflection and conjugation. The FourIER intensities and the coefficients of the three non-trivial solutions $x_{1}, x_{2}$, and $x_{3}$ are shown in Figure 6.2 on the following page. 


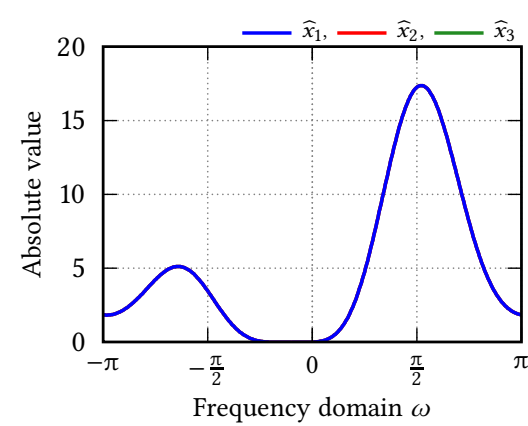

(a) FOURIER intensities of the signals $x_{1}, x_{2}$, and $x_{3}$

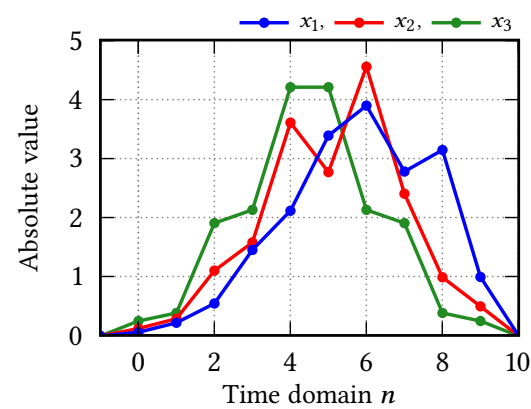

(c) Absolute value of the signals $x_{1}$, $x_{2}$, and $x_{3}$ illustrated by polygonal lines

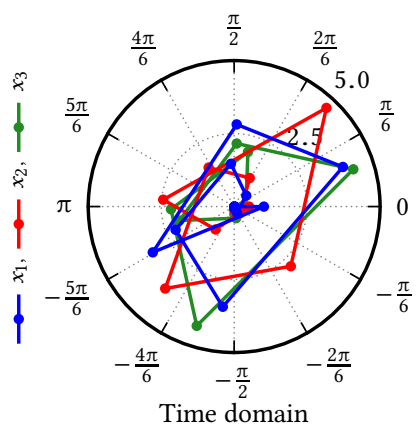

(b) Signals $x_{1}, x_{2}$, and $x_{3}$ represented in polar form

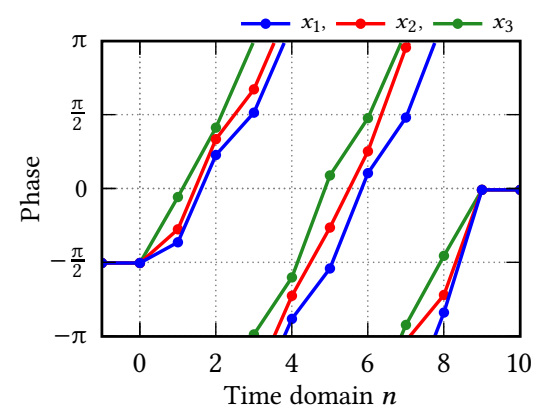

(d) Phase of the signals $x_{1}, x_{2}$, and $x_{3}$ illustrated by polygonal lines

Figure 6.2.: Discrete-time phase retrieval problem with exactly three nontrivial ambiguities

Example 6.7. In this last example, we finally consider a discrete-time phase retrieval problem with the full solution set of $2^{N-2}$ non-trivial ambiguities. Therefore, we want to recover the signal $x$ with modulus and phase given by

$$
|x|=\frac{1}{20}(\ldots, 0, \underline{20}, 25,40,28,24,20,26,32,18,5,0, \ldots)
$$

and

$$
\arg x[n]=\left(\cos \frac{4 \pi n}{9}\right)-1
$$

respectively, see Figure 6.3 on the next page. The corresponding zero set $\left\{\beta_{j}: j=\right.$ $1, \ldots, 9\}$ of the signal $x$ contains no zeros $\beta_{j}$ lying on the unit circle or pairs $\left(\beta_{j}, \bar{\beta}_{j}^{-1}\right)$ of reflected zeros at the unit circle as shown in Figure 6.3(e). Consequently, all zero pairs $\left(\gamma_{j}, \bar{\gamma}_{j}^{-1}\right)$ of the associated polynomial are pairwise distinct and arise with multiplicity one. With Proposition 6.1, the phase retrieval problem to recover the signal $x$ has $2^{8}=256$ non-trivial solutions. 


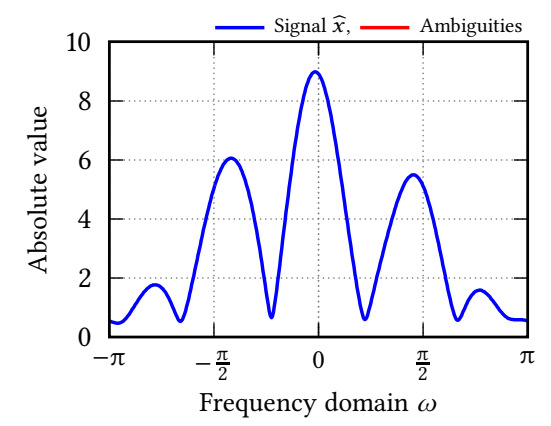

(a) FOURIER intensities of the signal $x$ and all non-trivial ambiguities

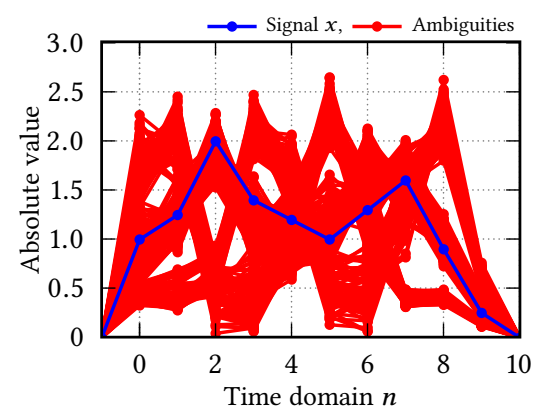

(c) Absolute value of the signal $x$ and all non-trivial ambiguities illustrated by polygonal lines

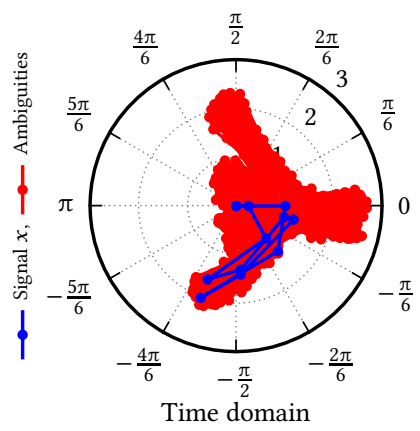

(b) Signal $x$ and all non-trivial ambiguities represented in polar form

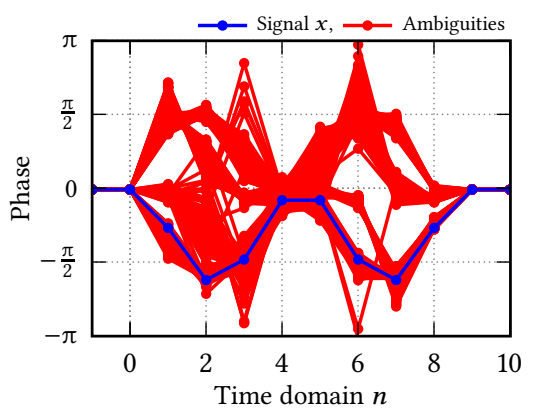

(d) Phase of the signal $x$ and all nontrivial ambiguities illustrated by polygonal lines

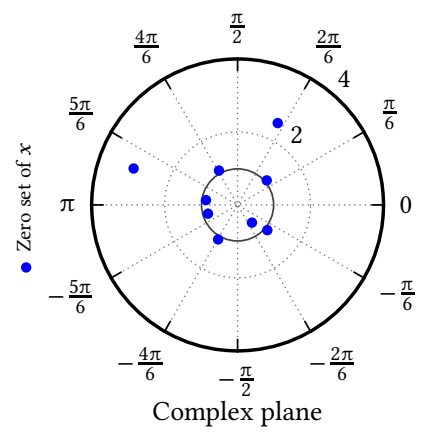

(e) Corresponding zero set of the original signal $x$

Figure 6.3.: Discrete-time phase retrieval problem with a full solution set of $2^{8}$ non-trivial ambiguities 
If we especially consider the modulus of all non-trivial ambiguities in Figure 6.3(c), we can observe that the non-trivial solutions occur in a wide range of different shapes. For instance, some signals are nearly zero around the points 2 and 6, unlike the signal $x$ itself. Since we are interested in the recovery of the original signal $x$, we need further a priori conditions or additional data in order to reduce the set of non-trivial ambiguities to an appropriate small set. 


\title{
Phase retrieval of non-negative signals
}

\begin{abstract}
In many applications, the components of the unknown signal can only be real-valued and non-negative. Although the additional non-negativity condition normally reduces the set of ambiguities, BRUCK and SodIN [BS79] and FiENUP [Fie78] have given some instances where the unknown signal cannot be recovered uniquely. Based on our characterization of the complete solution set of the discrete-time phase retrieval problem, we will examine how far the supposed non-negativity of the unknown signal can enforce the uniqueness of the problem. Inspired by the work of BRIGGS [Bri85] about positive polynomials, we will describe the influence of a single complex conjugated zero pair of the corresponding zero set on the non-negativity of the considered signal. Generalizing this approach to the complete solution set, we will finally observe that neither the signals that can be uniquely recovered nor the signals without a unique reconstruction form negligible sets. Therefore, in the one-dimensional discrete-time setting, the non-negativity condition is generally not sufficient to ensure unique solvability of the phase retrieval problem.

$\triangleleft$
\end{abstract}

\section{Non-negativity of a real signal}

Since the discrete-time phase retrieval problem is usually highly ambiguous, we need appropriate a priori conditions or additional data in order to recover the original signal from its FouRIER intensity. Although there are many approaches to incorporate further a priori information on the unknown signal in the literature on numerical algorithms for phase retrieval, theoretical considerations about the solvability with additional conditions occur rarely. Therefore, using the characterization of the ambiguities in Theorem 5.1, we investigate whether the usually applied a priori conditions can overcame the ambiguousness of the discrete-time phase retrieval problem or not.

One of the simplest ideas to enforce uniqueness of the phase retrieval problem is to restrict the corresponding zeros $\beta_{j}$, chosen from the zero pairs $\left(\gamma_{j}, \bar{\gamma}_{j}^{-1}\right)$ of 
the associated polynomial to the autocorrelation function, in Theorem 5.1 in an appropriate manner, cf. [HLO8o, Theorem 7, et seq.] for real-valued signals. For example, we may consider only signals whose corresponding zeros lie inside the unit circle; in other words, we assume that $\left|\beta_{j}\right| \leq 1$. Consequently, the zeros $\beta_{j}$ in Theorem 5.1 are uniquely determined, and hence the phase retrieval problem is uniquely solvable up to rotations and shifts. Alternatively, we may assume that all corresponding zeros lie outside the unit circle. Unfortunately, these restrictions are very arbitrary, and we are not aware of any physical meaning.

A further a priori condition which is often used in applications is to assume that the unknown signal with finite support is moreover real-valued and nonnegative. However, as already shown exemplarily in [BS79, Example 1, et seqq.] and [Fie78, Figure 2], the non-negativity cannot always ensure a unique recovery of the unknown signal. In the following, we will investigate the question whether non-negative signals that cannot be recovered uniquely are rare exceptions or the usual case. At first we give some examples which occur in the phase retrieval problem of non-negative signals.

Example 7.1. Ideally, the a priori condition that the unknown discrete-time signal is real and consists of non-negative components leads to a unique solution of the discrete-time phase retrieval problem. We give an explicit example that the non-negativity condition can ensure a unique recovery of a signal up to trivial ambiguities caused by shifts. For this purpose, we consider the discrete-time signal

$$
\begin{aligned}
x_{1}:=\frac{1}{4} \cdot\left(\delta_{1}\right. & \left.+\frac{3}{2} \delta_{0}\right) *\left(\delta_{1}+\left(\frac{1}{2}-\frac{3}{2} \mathrm{i}\right) \delta_{0}\right) *\left(\delta_{1}+\left(\frac{1}{2}+\frac{3}{2} \mathrm{i}\right) \delta_{0}\right) \\
& *\left(\delta_{1}-(1+\mathrm{i}) \delta_{0}\right) *\left(\delta_{1}-(1-\mathrm{i}) \delta_{0}\right) .
\end{aligned}
$$

Keeping in mind that the corresponding zeros of a real-valued signal arise in conjugate pairs, see Remark 4.10, we can construct three further non-trivially different signals with the same FoURIER intensity by Theorem 5.1, namely the signal $x_{2}$ by reflecting the real zero $-3 / 2$, the signal $x_{3}$ by reflecting the complex zero pair $-1 / 2 \pm 3 / 2 \mathrm{i}$, and, finally, the signal $x_{4}$ by reflecting $-3 / 2$ together with $-1 / 2 \pm 3 / 2 \mathrm{i}$ at the unit circle. Reflecting the remaining zero pair $1 \pm \mathrm{i}$ only leads to further trivial ambiguities caused by reflection, see Lemma 5.3. These three ambiguities and the original signal $x_{1}$ are presented in Figure 7.1 on the facing page. Obviously, the solutions $x_{2}, x_{3}$ and $x_{4}$ have at least one negative component; therefore, the phase retrieval problem to recover $x_{1}$ is uniquely solvable up to shifts.

Example 7.2. Unlike the phase retrieval problem in Example 7.1, the non-negativity constraint cannot in general ensure uniqueness of the phase retrieval prob- 


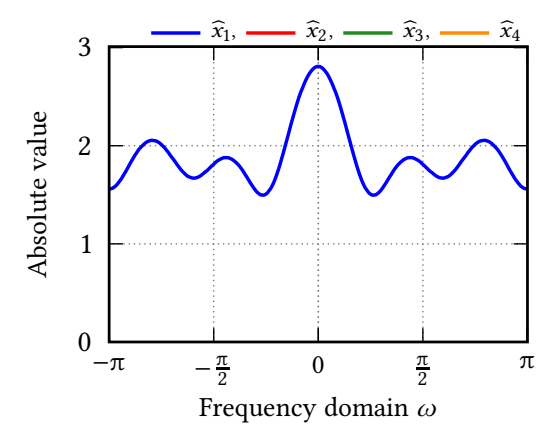

(a) Fourier intensities of the signals $x_{1}, x_{2}, x_{3}$, and $x_{4}$

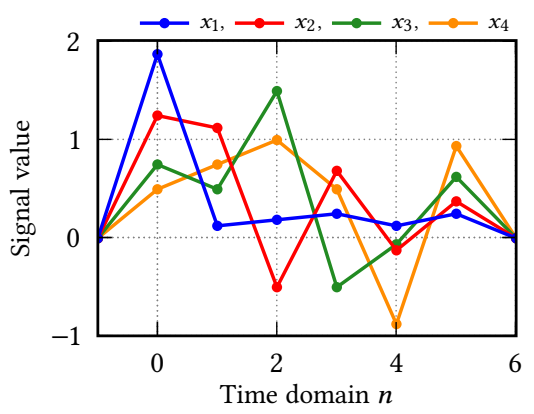

(b) Real solutions $x_{1}, x_{2}, x_{3}$, and $x_{4}$ illustrated by polygonal lines

Figure 7.1.: Discrete-time phase retrieval problem of non-negative signals with a unique solution up to shifts

lem, see for instance the examples by BRUCK and Sodin in [BS79] or by FIENUP in [Fie78] as mentioned before. However, in this example, we want to illustrate that sometimes the non-negativity does not reduce the set of ambiguities at all.

For this purpose, we consider the signal

$$
x:=\frac{1}{60} \cdot\left(\delta_{1}+\frac{5}{2} \delta_{0}\right) *\left(\delta_{1}+\frac{18}{5} \delta_{0}\right) *\left(\delta_{1}+\frac{6}{5} \delta_{0}\right) *\left(\delta_{1}+\frac{9}{5} \delta_{0}\right) *\left(\delta_{1}+\frac{7}{8} \delta_{0}\right) .
$$

In analogy to Example 6.7, the corresponding zeros $\beta_{j}$ are chosen in a way such that no reflected pairs at the unit circle appear. Hence, the phase retrieval problem to recover the signal $x$ has exactly $2^{4}=16$ non-trivially different solutions by Proposition 6.1. Moreover, these ambiguities are real-valued because each of the corresponding zeros is real. As shown in Figure 7.2 on the next page, the ambiguities are also non-negative, thus the set of non-trivial ambiguities is not reduced by the non-negativity constraint.

Example 7.3. In Proposition 3.4, we have shown that the FourIER intensity of a discrete-time signal is given by a non-negative trigonometric polynomial - the autocorrelation function. Moreover, each non-negative trigonometric polynomial is the autocorrelation function of some signals, see Corollary 4.9. In other words, the phase retrieval problem has at least one solution for every given nonnegative trigonometric polynomial. As discussed in Remark 4.10, this observation can be transferred to the real case, where the autocorrelation function is additionally even. However, the real-valued signals corresponding to a given FOURIER intensity do not have to be non-negative in general.

For instance, we examine the ambiguities of the phase retrieval problem with the Fourier intensity $|\widehat{x}|$ shown in Figure 7.3 on the following page. More de- 


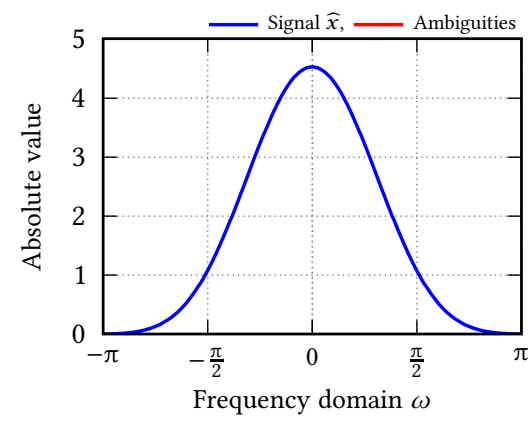

(a) FourIER intensities of the signal $x$ and all non-trivial ambiguities

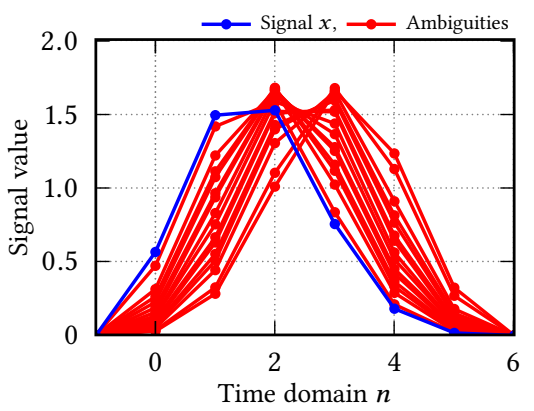

(b) Signal $x$ and all real non-trivial ambiguities illustrated by polygonal lines

Figure 7.2.: Discrete-time phase retrieval problem of non-negative signals with a full solution set of $2^{4}$ non-trivial ambiguities

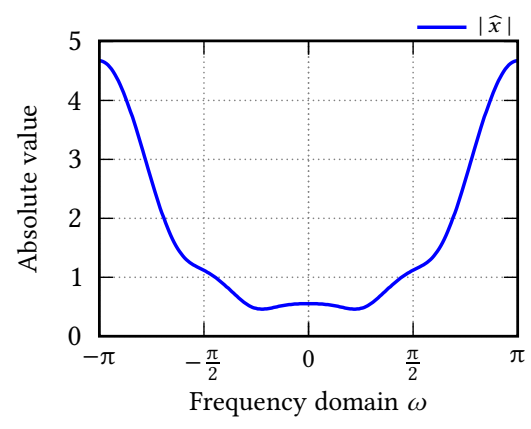

(a) FOURIER intensity of the unknown signals $x$

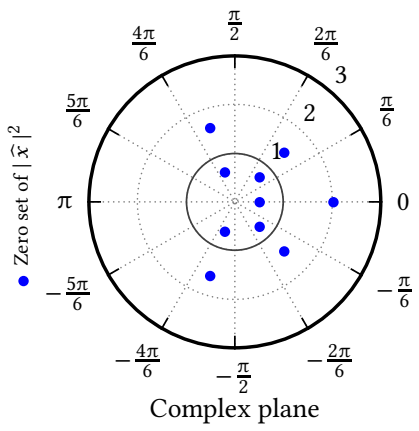

(b) Zero set of the associated polynomial to $|\widehat{x}|^{2}$

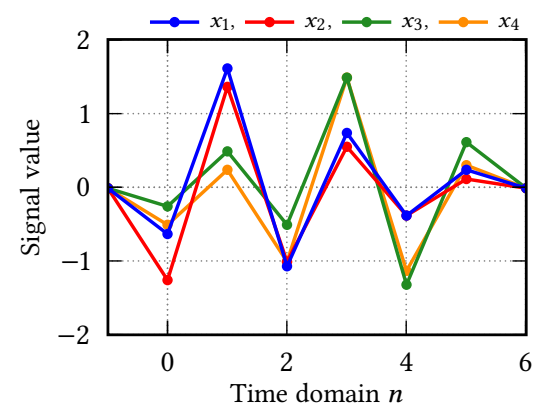

(c) Real solutions $x_{1}, x_{2}, x_{3}$, and $x_{4}$ illustrated by polygonal lines

Figure 7.3.: Discrete-time phase retrieval problem of real-valued signals without a non-negative solution 
tailed, in this example, the zeros of the associated polynomial to the autocorrelation function $|\widehat{x}|^{2}$ are

$$
\begin{array}{r}
\frac{1}{10}\{20,5,-5+15 i,-5-15 i,-\sqrt{10}(1+3 i),-\sqrt{10}(1-3 i), \\
10+10 i, 10-10 i, \sqrt{2}(5+5 i), \sqrt{2}(5-5 i)\},
\end{array}
$$

as shown in Figure 7.3(b). Here all zeros occur in pairs reflected at the unit circle with multiplicity one. Using the representation of the solutions in Theorem 5.1, we find four non-trivially different solutions. For the first signal $x_{1}$, we choose the five zeros $1 / 2,-1 / 2(1 \pm 3 i)$, and $1 \pm i$. Furthermore, we replace the real zero by 2 for the signal $x_{2}$ and the first complex pair by $-\sqrt{10} / 10(1 \pm 3 i)$ for the signal $x_{3}$. For the last solution $x_{4}$, we reflect the real zero and the first complex pair at the unit circle.

Obviously, all solutions in Figure 7.3(c) possess some negative components; so the considered phase retrieval problem cannot be solved by a non-negative signal. In other words, the non-negativity constraint can lead to an inconsistence with the given FOURIER intensity.

\section{Positivity of algebraic polynomials}

To answer the question whether a non-trivial solution of a discrete-time phase retrieval problem is non-negative or not, we will firstly investigate conditions depending on the corresponding zero set $\left\{\beta_{j}: j=1, \ldots, N-1\right\}$ which ensure that a real signal with finite support possesses only non-negative components. For this purpose, we will identify the real signal with the coefficients of an algebraic polynomial and use the well developed theory of polynomials.

Looking back at the characterization of the ambiguities in Theorem 5.1, we can write every solution $x$ to a certain phase retrieval problem in the form

$$
x=\mathrm{e}^{\mathrm{i} \alpha} \sqrt{|a[N-1]| \prod_{j=1}^{N-1}\left|\beta_{j}\right|^{-1}} \cdot\left[\delta_{n_{0}} * \underset{j=1}{\mathbb{*}}\left(\delta_{1}-\beta_{j} \delta_{0}\right)\right] .
$$

For the moment, we will neglect the multiplication with the prefactor and the convolution with $\delta_{n_{0}}$ because they merely cause a scaling and a shift respectively. Expanding the remaining non-trivial part of the solution signal $x$ yields 
the equality

$$
\underset{j=1}{*}\left(\delta_{1}-\beta_{j} \delta_{0}\right)=\sum_{n=0}^{N-1} c_{n} \delta_{n}
$$

with appropriate real coefficients $c_{n}$. Here the leading coefficient $c_{N-1}$ is equal to one; so the non-negativity of the considered signal only depends on the chosen zeros $\beta_{j}$. Moreover, we can conclude that the rotation $\mathrm{e}^{\mathrm{i} \alpha}$ in the prefactor must vanish in order to get a solution with non-negative components. The discretetime FoURIER transform of the non-trivial part of the solution $x$ is given by

$$
\prod_{j=1}^{N-1}\left(\mathrm{e}^{-\mathrm{i} \omega}-\beta_{j}\right)=\sum_{n=0}^{N-1} c_{n} \mathrm{e}^{-\mathrm{i} \omega n} .
$$

In analogy to the associated polynomial in Section 4 , the substitution $\mathrm{e}^{-\mathrm{i} \omega}=z$ now yields the algebraic polynomial

$$
\prod_{j=1}^{N-1}\left(z-\beta_{j}\right)=\sum_{n=0}^{N-1} c_{n} z^{n}
$$

with leading coefficient one and non-zero constant term. Hence, the signal $x$ possesses only non-negative components if and only if the monic polynomial with the zero set $\left\{\beta_{j}: j=1, \ldots, N-1\right\}$ has non-negative coefficients or, more precisely, is a positive polynomial as defined in [Bri85, p. 77].

Definition 8.1. An algebraic polynomial with real coefficients is called a positive polynomial if the leading coefficient and constant term are positive and if the remaining coefficients are non-negative.

\subsection{Dependency on the corresponding zero set}

Since the non-trivial ambiguities only depend on the set $\left\{\beta_{j}: j=1, \ldots, N-1\right\}$ chosen from the zeros of the associated polynomial, see Theorem 5.1, we are primarily interested in the relation between the zeros of a polynomial and its positivity. For some specific cases stated in [Bri85, p. 78], we can immediately decide whether a polynomial is positive or not by looking only at its zeros. For the first observation, we consider Descartes's rule of signs, see for instance [Obr63, Satz 13.1]. 
Theorem 8.2 (DESCARTES). The number of positive zeros of an algebraic polynomial with real coefficients is equal to the number of sign changes in the coefficient sequence or less than it by an even number.

Since all coefficients of a positive polynomial are at least non-negative and hence have the same sign, the number of sign changes in the coefficient sequence is zero. Hence, with Descartes's rule of signs, a positive polynomial cannot have real positive zeros, or conversely, if the zero set of a polynomial contains a real positive zero, then the polynomial cannot be positive.

Unfortunately, besides the real zeros, a polynomial with real coefficients usually possesses complex zeros in conjugate pairs, and the relation between the complex zeros and the positivity is not as obvious as for real zeros. However, if the zero set consist only of zeros with negative real part as considered in [Bri85, p. 78], we can easily verify that the corresponding monic polynomial is positive.

Proposition 8.3. If a monic polynomial with real coefficients only possesses zeros with negative real part, then the polynomial is positive.

Proof. We consider the linear and quadratic factors in the factorization of a real polynomial separately. First, let $\beta$ be a real negative zero, then the corresponding linear factor is of the form

$$
z-\beta
$$

and hence a positive polynomial. Now, let $\beta$ and $\bar{\beta}$ be a complex zero pair of the polynomial. The corresponding quadratic factor is

$$
(z-\beta)(z-\bar{\beta})=z^{2}-2 \mathfrak{R} \beta z+|\beta|^{2} .
$$

This is obviously a positive polynomial because the real part of $\beta$ is negative. Due to the fact that the product of positive polynomial is again a positive polynomial, the assertion follows.

Polynomials with real coefficients of this kind are an important subset of the positive polynomials and are named after Hurwitz, see for instance [Bri85, p. 78] or [Obr63, p. 108]. 
Definition 8.4. An algebraic polynomial with real coefficients is called a HURWITZ polynomial if the real part of all zeros is negative.

Example 8.5. Although every HuRwitz polynomial is positive polynomial, the reverse does not hold true. For example, we consider the positive polynomial

$$
z^{3}+z^{2}+2 z+8=(z+2)\left(z^{2}-z+4\right)
$$

see [Bri85, p. 78]. This polynomial is non-HuRwitzian because the real part of the complex zero pair $1 / 2(1 \pm \sqrt{15} \mathrm{i})$ is positive.

Now, the remaining part of our initial problem is to find appropriate conditions on the zeros lying in the right half plane such that the corresponding monic polynomial is positive. In order to investigate this question, we will use VIETA's formulae which relates the coefficients of a polynomial to the corresponding zero set by the elementary symmetric functions. More detailed, the elementary symmetric functions are given by the following definition, see for instance [GKN79, p. 422].

Definition 8.6. The elementary symmetric function $S_{n}$ of degree $n$ in the indeterminates $\beta_{1}, \ldots, \beta_{N-1}$ is defined by

$$
S_{n}\left(\beta_{1}, \ldots, \beta_{N-1}\right):=\sum_{1 \leq k_{1}<\cdots<k_{n} \leq N-1} \beta_{k_{1}} \cdots \beta_{k_{n}}
$$

for $n$ from 1 to $N-1$. Moreover, we define $S_{0}:=1$ and $S_{n}:=0$ for $n<0$ and $n \geq N$.

Using the elementary symmetric function, we can state VIETA's formulae, see for instance [GKN79, p. 478 et seq.], in the following manner.

Theorem 8.7 (VIETA). Let $P$ be a monic polynomial with the corresponding zero set $\left\{\beta_{1}, \ldots, \beta_{N-1}\right\}$. Then the polynomial $P$ can be written as

$$
P(z)=\sum_{n=0}^{N-1}(-1)^{n} S_{n}\left(\beta_{1}, \ldots, \beta_{N-1}\right) z^{N-1-n} .
$$




\subsection{Constraints for the last conjugate zero pair}

In order to find the dependency of the positivity of a polynomial on the complex zeros, we follow the approach of BRIGGS in [Bri85]. This means that we fix all zeros in a zero set except for one complex conjugate pair and investigate the positivity of the corresponding monic polynomial depending on this free zero pair. This consideration leads to the following conditions that are generalizations of the findings in [Bri85, p. 85 et seq.], where all fixed zeros are real, and where the free complex zero pair lies in the right half plane.

Theorem 8.8. Let $P$ be a monic polynomial with real coefficients corresponding to the zero set $\left\{\beta_{1}, \ldots, \beta_{N-1}\right\}$. Assume that $\beta_{N-2}$ and $\beta_{N-1}$ are a conjugate zero pair unequal to zero, and define $\sigma_{n}:=(-1)^{n} S_{n}\left(\beta_{1}, \ldots, \beta_{N-3}\right)$ for every integer $n$. Then $P$ is a positive polynomial if and only if the zero $\beta_{N-1}$ fulfils

$$
\sigma_{n-2}\left|\beta_{N-1}\right|^{2}-2 \sigma_{n-1} \mathfrak{R} \beta_{N-1}+\sigma_{n} \geq 0
$$

for $n$ from 0 to $N-1$, and if $\sigma_{N-3}$ is non-zero.

Proof. We consider the zero set $\left\{\beta_{1}, \ldots, \beta_{N-1}\right\}$ of a polynomial with real coefficients where the last two zeros $\beta_{N-2}$ and $\beta_{N-1}$ form a conjugate zero pair. For that reason, the factorization of the monic polynomial $P$ can be written as

$$
P(z)=\left(z-\beta_{N-1}\right)\left(z-\bar{\beta}_{N-1}\right) \prod_{j=1}^{N-3}\left(z-\beta_{j}\right)
$$

Since the product over the first $N-3$ zeros of the polynomial $P$ is itself a monic polynomial, we can apply VIETA's formulae (Theorem 8.7) to it. Denoting the coefficients $(-1)^{n} S_{n}\left(\beta_{1}, \ldots, \beta_{N-3}\right)$ in ViETA's formulae by $\sigma_{n}$ and expanding the first two linear factor yields

$$
P(z)=\left(z^{2}-2 \mathfrak{R} \beta_{N-1} z+\left|\beta_{N-1}\right|^{2}\right)\left(\sum_{n=0}^{N-3} \sigma_{n} z^{N-3-n}\right)
$$

and a further expansion of this product leads to

$$
P(z)=\sum_{n=0}^{N-3} \sigma_{n} z^{N-1-n}-2 \Re \beta_{N-1} \sum_{n=0}^{N-3} \sigma_{n} z^{N-2-n}+\left|\beta_{N-1}\right|^{2} \sum_{n=0}^{N-3} \sigma_{n} z^{N-3-n} .
$$


Since $\sigma_{n}$ is equal to zero for negative $n$ and for $n$ greater then $N-3$, we can simplify this equation to

$$
P(z)=\sum_{n=0}^{N-1}\left(\sigma_{n}-2 \sigma_{n-1} \mathfrak{R} \beta_{N-1}+\sigma_{n-2}\left|\beta_{N-1}\right|^{2}\right) z^{N-1-n}
$$

by shifting the summation indices appropriately.

Considering the last representation of the polynomial $P$, we can conclude that $P$ has non-negative coefficients if and only if the last zero $\beta_{N-1}$ fulfils the inequalities

$$
\sigma_{n-2}\left|\beta_{N-1}\right|^{2}-2 \sigma_{n-1} \mathfrak{R} \beta_{N-1}+\sigma_{n} \geq 0
$$

for $n$ from 0 to $N-1$. In order to be a positive polynomial, the leading coefficient and the constant term of $P$ have to be positive. Since the leading coefficient of a monic polynomial is one by definition, it is sufficient to consider the constant term. Now, VIETA's formulae implies that the constant term of $P$ is given by $(-1)^{N-1} \beta_{1} \cdots \beta_{N-1}$. Since the conjugate zero pair $\left(\beta_{N-1}, \bar{\beta}_{N-1}\right)$ is already non-zero, the constant term is positive if and only if the product $\sigma_{N-3}$ over the remaining zeros is non-zero, which completes the proof.

Remark 8.9. The inequality condition

$$
\sigma_{n-2}\left|\beta_{N-1}\right|^{2}-2 \sigma_{n-1} \mathfrak{R} \beta_{N-1}+\sigma_{n} \geq 0
$$

for the last zero $\beta_{N-1}$ in Theorem 8.8 is satisfied for all $\beta_{N-1}$ in a disc, possibly degenerate, on the RiEmANn sphere, see for instance [FL12, p. 85]. Hence, the last zero $\beta_{N-1}$ must be contained in the intersection of $N$ discs or half planes on the complex plane to ensure that the corresponding monic polynomial is positive.

Depending on $\sigma_{n-2}, \sigma_{n-1}$, and $\sigma_{n}$, we distinguish three major cases. Firstly, let us assume that $\sigma_{n-2}$ is non-zero and additionally positive. Now, dividing the inequality for the last zero (8.2) by $\sigma_{n-2}$ and reorganizing the terms leads to the inequality

$$
\left|\beta_{N-1}\right|^{2}-2 \frac{\sigma_{n-1}}{\sigma_{n-2}} \mathfrak{R} \beta_{N-1} \geq-\frac{\sigma_{n}}{\sigma_{n-2}} .
$$

Completing the square by $\left(\sigma_{n-1} / \sigma_{n-2}\right)^{2}$, we finally have the condition

$$
\left|\beta_{N-1}-\frac{\sigma_{n-1}}{\sigma_{n-2}}\right|^{2} \geq \frac{\sigma_{n-1}^{2}-\sigma_{n} \sigma_{n-2}}{\sigma_{n-2}^{2}} .
$$

In this specific case, the last zero $\beta_{N-1}$ must hence lie on or outside the circle with centre $\sigma_{n-1} / \sigma_{n-2}$ and radius $\sqrt{\sigma_{n-1}^{2}-\sigma_{n} \sigma_{n-2}} / \sigma_{n-2}$. If the radius is imaginary, which 
means that $\sigma_{n-1}^{2}<\sigma_{n} \sigma_{n-2}$, then the inequality is trivially fulfilled, and the last zero $\beta_{N-1}$ can be chosen arbitrary. Assuming that $\sigma_{n-2}$ is negative instead of positive, we have to invert the circle inequality, and the last zero $\beta_{N-1}$ must lie on or inside the circle. If the radius is imaginary, then the inequality cannot be fulfilled.

Secondly, we consider the case where $\sigma_{n-2}$ is equal to zero, and where $\sigma_{n-1}$ is non-zero. Now, the first term in the inequality (8.2) vanishes, and the inequality reduces to

$$
2 \sigma_{n-1} \mathfrak{R} \beta_{N-1} \leq \sigma_{n}
$$

This condition implies that the last zero $\beta_{N-1}$ has to be contained in the closed half plane left or right of the imaginary axis through $\sigma_{n} / 2 \sigma_{n-1}$ depending on the sign of $\sigma_{n-1}$.

Finally, let us assume that both $\sigma_{n-2}$ and $\sigma_{n-1}$ are zero. Consequently, the inequality condition (8.2) degenerates to the constraint

$$
\sigma_{n} \geq 0,
$$

which is completely independent of the last zero $\beta_{N-1}$. Hence, the fixed zeros $\beta_{1}, \ldots, \beta_{N-3}$ must themselves fulfil some consistency constraints and cannot be chosen arbitrarily.

Example 8.10. The inequality conditions in Theorem 8.8 give us the opportunity to extend a given set of fixed zeros by an additional conjugate zero pair in order to construct a positive polynomial or a non-negative discrete-time signal. Unfortunately, this extension cannot always be done successfully since the fixed zeros also have to fulfil some consistency conditions as mentioned in Remark 8.9. In this example, we use the findings in Theorem 8.8 to construct a positive polynomial. More detailed, beginning with the three fixed zeros

$$
\beta_{1}:=-\frac{5}{2}, \quad \beta_{2}:=1+\mathrm{i}, \quad \text { and } \quad \beta_{3}:=1-\mathrm{i},
$$

we want to find a further conjugate zero pair that ensures the positivity of the corresponding monic polynomial.

In order to examine the inequality conditions in Theorem 8.8 for these fixed zeros, we need the constants $\sigma_{n}$ defined by $(-1)^{n} S_{n}\left(\beta_{1}, \beta_{2}, \beta_{3}\right)$ for all integer $n$. If we interpret these constants as coefficients of the monic polynomial with zeros $\beta_{1}, \beta_{2}$, and $\beta_{3}$, cf. VIETA's formulae in Theorem 8.7, then the expansion of the corresponding linear and quadratic factor

$$
\left(z+\frac{5}{2}\right)\left(z^{2}-2 z+2\right)=z^{3}+\frac{1}{2} z^{2}-3 z+5
$$




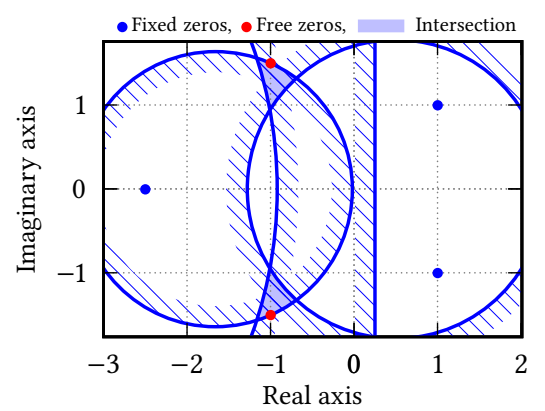

(a) Restrictions on the last zero pair to ensure positivity

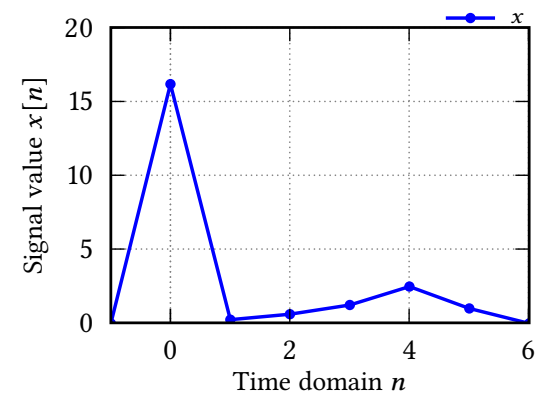

(b) Corresponding coefficient sequence $c$ or discrete-time signal $x$ illustrated by a polygonal line

Figure 8.1.: Restriction on the last zero pair in order to ensure positivity of the corresponding monic polynomial

yields

$$
\sigma_{0}=1, \quad \sigma_{1}=\frac{1}{2}, \quad \sigma_{2}=-3, \quad \text { and } \quad \sigma_{4}=5 .
$$

As before, $\sigma_{n}$ is zero for $n<0$ and $n>4$.

Now, for $n=0$, the corresponding condition (8.1) in Theorem 8.8 is simply

$$
\sigma_{0}=1 \geq 0
$$

which is always fulfilled. For $n=1$, the condition (8.1) becomes

$$
-2 \mathfrak{R} \beta_{5}+\frac{1}{2} \geq 0 \text {. }
$$

Hence, the last zero pair has to lie in the closed half plane left of the imaginary axis through $1 / 4$. Further, for $n=2$ and $n=3$, we have the inequalities

$$
\left|\beta_{5}\right|^{2}-\mathfrak{R} \beta_{5}-3 \geq 0 \quad \text { and } \quad \frac{1}{2}\left|\beta_{5}\right|^{2}+6 \mathfrak{R} \beta_{5}+5 \geq 0
$$

As discussed in Remark 8.9, we must choose the last zero pair on or outside the two circles with centres $1 / 2$ and -6 in the complex plane and radii $\sqrt{13} / 2$ and $\sqrt{26}$ respectively. For $n=4$, the inequality condition is

$$
-3\left|\beta_{5}\right|^{2}-10 \Re \beta_{5} \geq 0 \text {. }
$$

Since the leading coefficient is negative, the last zero must lie on or inside the 
circle with centre $-5 / 2$ and radius $5 / 3$, see Remark 8.9. Finally, for $n=5$, we have

$$
5\left|\beta_{5}\right|^{2} \geq 0
$$

which is fulfilled whenever $\beta_{5}$ is non-zero.

The half plane and the three circles are visualized in Figure 8.1 on the preceding page. Choosing the last conjugate zero pair, for example

$$
\beta_{4}:=-1+\frac{3}{2} \mathrm{i} \quad \text { and } \quad \beta_{5}:=-1-\frac{3}{2} \mathrm{i},
$$

in the intersection of the found restrictions ensures that the corresponding monic polynomial with the coefficient sequence

$$
c=\frac{1}{8}(\ldots, 0, \underline{130}, 2,5,10,20,8,0, \ldots)
$$

is a positive polynomial of degree five. Moreover, if we renounce any additional shift or multiplicative constant, we can interpret the coefficients sequence $c$ as the non-negative discrete-time signal $x$ presented in Figure 8.1(b) with the corresponding zero set $\left\{\beta_{1}, \ldots, \beta_{5}\right\}$.

As seen in Remark 8.9 and Example 8.10, the inequality conditions in Theorem 8.8 for the last zero pair can have a wide range of different manifestations. However, if we restrict the fixed zeros in an appropriate manner, we can simplify these conditions. For example, restricting the zero set such that all fixed zeros have a purely negative real part lead to a complex version of the findings by BRIGGS in [Bri85, Section 7], where the last zero pair has to lie in a certain half plane and, further, on or outside certain circles.

Corollary 8.11. Let $P$ be a monic polynomial with real coefficients corresponding to the zero set $\left\{\beta_{1}, \ldots, \beta_{N-1}\right\}$. Assume that the real part of $\beta_{j}$ is negative for $j$ from 1 to $N-3$, and that $\beta_{N-2}$ and $\beta_{N-1}$ are a conjugate zero pair unequal to zero. Further, define $\sigma_{n}:=(-1)^{n} S_{n}\left(\beta_{1}, \ldots, \beta_{N-3}\right)$ for every integer $n$. Then $P$ is a positive polynomial if and only if the last zero $\beta_{N-1}$ lies in the closed half plane left of the imaginary axis through $\sigma_{1} / 2$ and, moreover, on or outside the circles with centre and radius

$$
\frac{\sigma_{n-1}}{\sigma_{n-2}} \text { and } \quad \frac{\sqrt{\sigma_{n-1}^{2}-\sigma_{n} \sigma_{n-2}}}{\sigma_{n-2}}
$$

for $n$ from 2 to $N-2$ whenever the radius is real. 
Proof. Since all real parts of the fixed zeros $\beta_{1}, \ldots, \beta_{N-3}$ are negative, the corresponding linear and quadratic factors are of the form

$$
z-\beta_{j} \quad \text { and } \quad z^{2}-2 \Re \beta_{j}+\left|\beta_{j}\right|^{2}
$$

for real zeros $\beta_{j}$ and conjugate zero pairs $\left(\beta_{j}, \bar{\beta}_{j}\right)$ respectively. Due to the fact that all coefficients of these factors are purely positive, the product over these factors is a polynomial with positive coefficients.

Remembering that the constants $\sigma_{n}:=(-1)^{n} S_{n}\left(\beta_{1}, \ldots, \beta_{N-3}\right)$ are the coefficients of the monic polynomial corresponding to the zero set $\left\{\beta_{1}, \ldots, \beta_{N-3}\right\}$ by VIETA's formulae in Theorem 8.7, we can conclude that the constants $\sigma_{n}$ for $n$ from 0 to $N-3$ are positive, and that the remaining constants $\sigma_{n}$ are zero. Now, we investigate the restrictions for the last zero in Theorem 8.8 for different integer $n$. Firstly, for $n=0$, we have

$$
\sigma_{0}=1 \geq 0
$$

by definition. Secondly, for $n=1$ the inequality is reduced to

$$
2 \Re \beta_{N-1} \leq \sigma_{1}
$$

Hence, the last zero $\beta_{N-1}$ has to lie in the half plane left of the axis through $\sigma_{1} / 2$. Next, for $n$ from 2 to $N-2$, the condition

$$
\sigma_{n-2}\left|\beta_{N-1}\right|^{2}-2 \sigma_{n-1} \mathfrak{R} \beta_{N-1}+\sigma_{n} \geq 0
$$

with positive coefficients $\sigma_{n}$ implies that $\beta_{N-1}$ must lie on or outside the circle with centre $\sigma_{n-1} / \sigma_{n-2}$ and radius $\sqrt{\sigma_{n-1}^{2}-\sigma_{n} \sigma_{n-2}} / \sigma_{n-2}$ as discussed in Remark 8.9. Finally, for $n=N-1$, we have

$$
\sigma_{N-3}\left|\beta_{N-1}\right|^{2} \geq 0
$$

This condition is trivially fulfilled since the two factors on the left-hand side are positive due to the assumptions.

Example 8.12. Analogously as in Example 8.10, we apply Corollary 8.11 to construct a positive polynomial by choosing a further conjugate zero pair beside the three fixed zeros

$$
\beta_{1}:=-\frac{3}{2}, \quad \beta_{2}:=-1+\mathrm{i}, \quad \text { and } \quad \beta_{3}:=-1-\mathrm{i}
$$




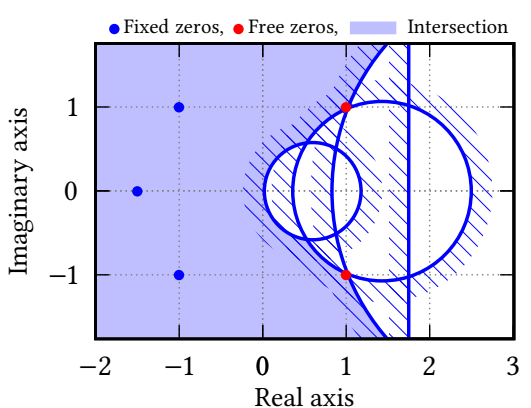

(a) Restriction on the last zero pair to ensure positivity

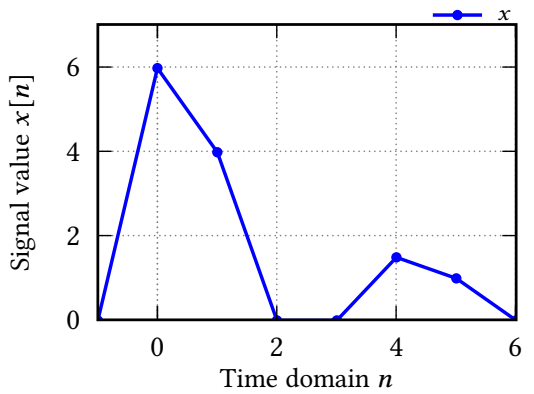

(b) Corresponding coefficient sequence $c$ or discrete-time signal $x$ illustrated by a polygonal line

Figure 8.2.: Restriction on the last zero pair in order to ensure positivity of the corresponding monic polynomial for fixed zeros with negative real part

with negative real part. For this specific zero set, the non-zero constants $\sigma_{n}:=$ $(-1)^{n} S_{n}\left(\beta_{1}, \beta_{2}, \beta_{3}\right)$ defined in Corollary 8.11 are given by

$$
\sigma_{0}=1, \quad \sigma_{1}=\frac{7}{2}, \quad \sigma_{2}=5, \quad \text { and } \quad \sigma_{4}=3 .
$$

Now, Corollary 8.11 implies that we must choose the further conjugate pair in the half plane left of the axis through $7 / 4$ and, moreover, on or outside the three circles with centres $7 / 2,10 / 7$, and $3 / 5$ in the complex plane and radii $\sqrt{29} / 2$, $\sqrt{58} / 7$, and $3 / 5$ respectively. These restrictions on the last zero pair are visualized in Figure 8.2. If we choose the last zero pair as

$$
\beta_{4}:=1+\mathrm{i} \quad \text { and } \quad \beta_{5}:=1-\mathrm{i},
$$

then the corresponding monic polynomial with the coefficients sequence

$$
c=\frac{1}{2}(\ldots, 0, \underline{12}, 8,0,0,3,2,0, \ldots)
$$

is obviously positive. Since the zero pair $\left(\beta_{4}, \beta_{5}\right)$ is exactly the intersection of two circles, the corresponding coefficients $c_{2}$ and $c_{3}$ vanish. Analogously to Example 8.10, we can interpret the coefficients sequence $c$ as discrete-time signal $x$ with the corresponding zero set $\left\{\beta_{1}, \ldots, \beta_{5}\right\}$.

Remark 8.13. In Corollary 8.11, we assume that the real parts of the fixed zeros $\beta_{1}, \ldots, \beta_{N-3}$ are negative. If we now extend the fixed zero set by a further conjugate zero pair with negative real part, the corresponding polynomial $P$ obviously 
is a HuRwitz polynomial and hence positive. This specific behavior is directly reflected by the positivity constraints in Corollary 8.11. More precisely, since the constants $\sigma_{n}$ are positive for $n$ from 0 to $N-3$, the imaginary axis through $\sigma_{1} / 2$ and the considered circles are completely contained in the right half plane.

\section{Non-negative solutions of the phase retrieval problem}

A single solution $x$ of a certain phase retrieval problem is non-negative if and only if the monic polynomial $\prod_{j=1}^{N-1}\left(z-\beta_{j}\right)$, where $\beta_{j}$ denotes the corresponding zeros of the signal $x$, is positive as discussed in Section 8. However, in order to decide whether the non-negativity condition can in general enforce the uniqueness of the phase retrieval problem or can at least reduce the set of arising ambiguities, we need to investigate the non-negativity of the whole set of nontrivial solutions. In the end, we will show that neither the uniqueness nor the occurrence of several non-trivial solutions are a rare exceptions.

\subsection{Non-negativity of non-trivially different ambiguities}

We start our investigation with a simple special case. If the unknown signal possesses a corresponding zero set with purely negative real parts, then we can show that all arising non-trivial ambiguities of the corresponding phase retrieval problem are non-negative too. Hence, the non-negativity restriction cannot reduce the set of ambiguities in this case.

Proposition 9.1. Let $x$ be a real-valued discrete-time signal with finite support. If the corresponding zero set is contained in the left half plane, then all occurring real-valued non-trivial ambiguities of the corresponding phase retrieval problem are non-negative.

Proof. Using Theorem 5.1, we can characterize the non-trivial ambiguities of a phase retrieval problem by choosing the corresponding zeros from the zero pairs $\left(\gamma_{j}, \bar{\gamma}_{j}^{-1}\right)$ of the associated polynomial. Based on the corresponding zeros $\beta_{j}$ of the considered signal $x$, we can hence generate all non-trivial ambiguities by reflecting some of the real zeros or conjugate zero pairs at the unit circle. Since the real part of a reflected zero is obviously given by

$$
\mathfrak{R} \bar{\beta}_{j}^{-1}=\frac{1}{\left|\beta_{j}\right|^{2}} \mathfrak{R} \beta_{j}
$$


replacing a zero by its reflection again leads to a zero set contained in the left half plane. Consequently, the monic polynomials to these zero sets are Hurwitzian and hence positive, which implies that the corresponding signals are non-negative.

Once more, the question arises: how does the non-negativity of the complete solution set of a phase retrieval problem depend on conjugate zero pairs in the right half plane? As for a single discrete-time signal or monic polynomial, this dependency is not obvious. Nonetheless, we can generalize our findings about the restrictions on a chosen corresponding zero pair to ensure non-negativity of a signal in Section 8.2.

Based on the corresponding zero set of a discrete-time signal with finite support, we can explicitly construct all occurring ambiguities of the phase retrieval problem to recover this signal by reflecting individual real zeros or conjugate zero pairs at the unit circle, cf. Theorem 5.1. Here the only trivial ambiguity that can appear is the reflection of a signal, which corresponds to the reflection of the complete zero set at the unit circle, see Lemma 5.3. We can avoid this trivial ambiguity by excepting one real zero or complex zero pair from the reflection. Considering the remaining zero sets, we can now use Theorem 8.8 and Corollary 8.11 to formulate restrictions on the excepted zero pair to ensure the non-negativity of certain non-trivial solutions.

Example 9.2. Beginning with our observations in Example 8.12, we wish to extend the zero set

$$
\Lambda:=\left\{-\frac{3}{2},-1+\mathrm{i},-1-\mathrm{i}\right\}
$$

by a further conjugate zero pair $\left(\beta_{4}, \beta_{5}\right)$ in the right half plane in order to construct a non-negative discrete-time signal $x$ with finite support. Simultaneously, we want to choose this zero pair such that most of the non-trivial ambiguities in the phase retrieval problem to recover the signal $x$ from its FOURIER intensity are non-negative too. As already observed in Example 8.12, the signal $x$ consists of non-negative components if and only if the further zero pair lies in the half plane left from the imaginary axis through $7 / 4$ and, moreover, on or outside the three circles with centres $7 / 2,10 / 7$, and $3 / 5$ in the complex plane and radii $\sqrt{29} / 2$, $\sqrt{58} / 7$, and $3 / 5$ respectively.

Due to the fact that the set $\Lambda$ consists of a real zero and a conjugate zero pair, the phase retrieval problem can at most have three further non-trivial ambiguities as discussed in Remark 6.2. Since we exclude the additional conjugate zero pair $\left(\beta_{4}, \beta_{5}\right)$ from the reflection at the unit circle in order to avoid trivial reflec- 
tions, the non-trivial ambiguities correspond to zero sets where we reflect either the real zero or the complex zero pair of $\Lambda$, or both.

For the first ambiguity $y_{1}$, we reflect the real zero $-3 / 2$ at the unit circle. This means that the solution $y_{1}$ of the phase retrieval problem corresponds to the zero set

$$
M_{1}:=\left\{-\frac{2}{3},-1+\mathrm{i},-1-\mathrm{i}\right\} .
$$

Since the real part of all zeros is negative, we can use Corollary 8.11 to formulate conditions for the non-negativity of this signal. In so doing, we have to choose the last conjugate zero pair in the half plane left from the imaginary axis through $4 / 3$ and, further, on or outside the circles with centres $8 / 3,5 / 4$, and $2 / 5$ in the complex plane and radii $\sqrt{34} / 3, \sqrt{17} / 4$, and $2 / 5$ respectively.

For the next ambiguity $y_{2}$, we reflect the complex zero pair $-1 \pm i$. Hence, the signal $y_{2}$ corresponds to the zero set

$$
M_{2}:=\left\{-\frac{3}{2},-\frac{1}{2}(1+\mathrm{i}),-\frac{1}{2}(1-\mathrm{i})\right\} .
$$

Using Corollary 8.11 once more, we must ensure that the last zero pair lies in the half plane left from the imaginary axis through $5 / 4$ and, moreover, on or outside the three circles with centres $5 / 2,4 / 5$, and $3 / 8$ in the complex plane and radii $\sqrt{17} / 2$, $\sqrt{850} / 50$, and $3 / 8$ respectively.

Now, the last non-trivial ambiguity $y_{3}$ corresponds to the zero set

$$
M_{3}:=\left\{-\frac{2}{3},-\frac{1}{2}(1+\mathrm{i}),-\frac{1}{2}(1-\mathrm{i})\right\},
$$

where we reflect the complete set $\Lambda$ at the unit circle. In order to ensure nonnegativity of this signal, the last zero pair has to be contained in the half plane left from the imaginary axis through $5 / 6$ and, further, on or outside the cumbersome circles with centres $5 / 3,7 / 10$, and $2 / 7$ in the complex plane and radii $\sqrt{522} / 18, \sqrt{2900} / 100$, and $2 / 7$ respectively.

The restrictions on the additional zero pair are visualized in Figure 9.1 on the next page. More detailed, Figure 9.1(a) shows the intersection of the half plane and the complements of the three discs for the fixed zeros in $\Lambda, M_{1}, M_{2}$, and $M_{3}$. Choosing the last conjugate zero pair $\left(\beta_{4}, \beta_{5}\right)$ in one or more intersections, we can ensure the non-negativity for certain non-trivial solutions and can directly influence the number of the occurring non-negative solutions.

In this example, we extend the original zero set $\Lambda$ by the conjugate zero pair $\left(\beta_{4}, \beta_{5}\right)$ with

$$
\beta_{4}:=\frac{3}{4}+\mathrm{i} \quad \text { and } \quad \beta_{5}:=\frac{3}{4}-\mathrm{i} \text {, }
$$




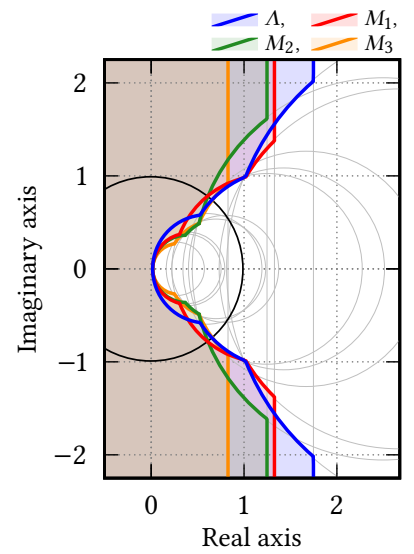

(a) Restrictions on the last conjugate zero pair to ensure positivity of the signals $x, y_{1}$, $y_{2}$, and $y_{3}$

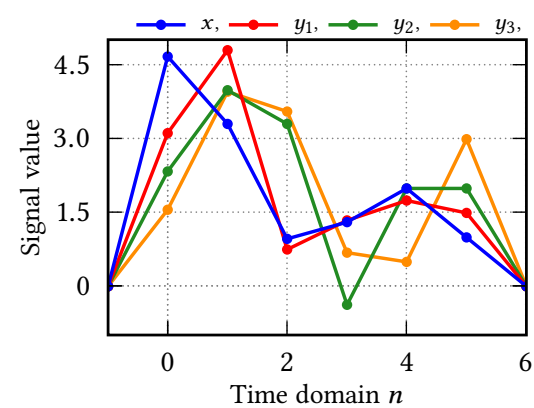

(b) Signals $x, y_{1}, y_{2}$, and $y_{3}$ illustrated by polygonal lines

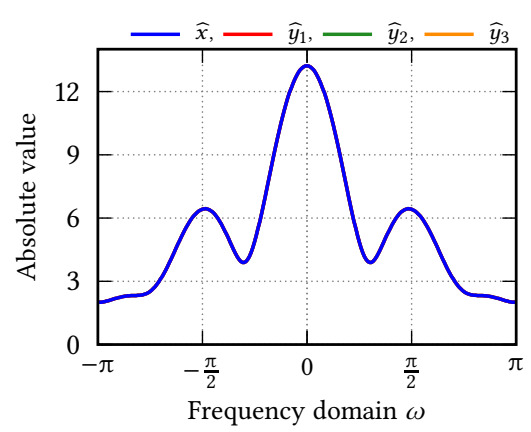

(c) FourIER intensities of the signals $x, y_{1}, y_{2}$, and $y_{3}$

Figure 9.1.: Restriction on the last zero pair in order to ensure nonnegativity of different non-trivial ambiguities

which fulfils the non-negativity constraints for $\Lambda, M_{1}$, and $M_{3}$. The non-negativity constraints for $M_{2}$ are nevertheless violated since the additional zero pair lies inside the circle with centre $5 / 2$ and radius $\sqrt{17} / 4$.

Based on the extended zero set $\Lambda$, we define the discrete-time signal $x$ by

$$
\begin{aligned}
x:=\left(\delta_{1}+\frac{3}{2} \delta_{0}\right) *( & \left.\delta_{1}+(1+\mathrm{i}) \delta_{0}\right) *\left(\delta_{1}+(1-\mathrm{i}) \delta_{0}\right) \\
& *\left(\delta_{1}-\left(\frac{3}{4}+\mathrm{i}\right) \delta_{0}\right) *\left(\delta_{1}-\left(\frac{3}{4}-\mathrm{i}\right) \delta_{0}\right) .
\end{aligned}
$$

Expanding this convolution leads to the representation

$$
x=\frac{1}{32}(\ldots, 0, \underline{150}, 106,31,42,64,32,0, \ldots),
$$

which is a non-negative signal as wanted. In order to determine the further nontrivial solutions of the phase retrieval problem, we again use Theorem 5.1. Based 
on the extended zero sets $M_{1}, M_{2}$, and $M_{3}$, we have the non-trivial ambiguities

$$
\begin{aligned}
& y_{1}=\frac{1}{32}(\ldots, 0, \underline{100}, 154,24,43,56,48,0, \ldots), \\
& y_{2}=\frac{1}{32}(\ldots, 0, \underline{75}, 128,106,-12,64,64,0, \ldots),
\end{aligned}
$$

and

$$
y_{3}=\frac{1}{32}(\ldots, 0, \underline{50}, 127,144,22,16,96,0, \ldots)
$$

respectively. All four signals and the corresponding discrete-time FOURIER intensities are shown in Figure 9.1(b) and Figure 9.1(c). Obviously, the signals $y_{1}$ and $y_{3}$ are also non-negative.

If we consider the restrictions on the last conjugate zero pair to ensure nonnegativity of the constructed signal in Example 9.2 and especially in Figure 9.1, it seems that the sets of all possible choices to extend the fixed zeros in $\Lambda$ and $M_{3}$ are reflections at the unit circle of each other. An analogous observation holds for the fixed zeros in $M_{1}$ and $M_{2}$. In order to show this behaviour formally, we need the following identity for the elementary symmetric functions.

Lemma 9.3. Let $\left\{\beta_{1}, \ldots, \beta_{N-1}\right\}$ be a multiset of non-zero complex numbers where the complex elements occur in conjugate pairs. Then the elementary symmetric functions fulfil the equality

$$
\frac{1}{\beta_{1} \cdots \beta_{N-1}} S_{n}\left(\beta_{1}, \ldots, \beta_{N-1}\right)=S_{N-1-n}\left(\bar{\beta}_{1}^{-1}, \ldots, \bar{\beta}_{N-1}^{-1}\right)
$$

for every integer $n$.

Proof. Firstly, we assume that $n$ is an integer between 1 and $N-2$. Using Definition 8.6 of the elementary symmetric functions, we have

$$
\frac{1}{\beta_{1} \cdots \beta_{N-1}} S_{n}\left(\beta_{1}, \ldots, \beta_{N-1}\right)=\frac{1}{\beta_{1} \cdots \beta_{N-1}} \sum_{\substack{1 \leq k_{1}<\cdots<k_{n} \leq N-1 \\ k_{1}}} \beta_{k_{n}} .
$$

On the right-hand side, we take the sum over the products of the subsets with $n$ elements of the multiset $\left\{\beta_{1}, \ldots, \beta_{N-1}\right\}$. If we multiply the summands with the prefactor, we summarize the products of the complements of the subsets with $n$ elements of the multiset $\left\{\beta_{1}^{-1}, \ldots, \beta_{N-1}^{-1}\right\}$. In other words, we take the sum over the products of the subsets with $N-1-n$ elements since each subset has a unique complement. 
This observation leads us to the equality

$$
\frac{1}{\beta_{1} \cdots \beta_{N-1}} S_{n}\left(\beta_{1}, \ldots, \beta_{N-1}\right)=\sum_{1 \leq k_{1}<\cdots<k_{N-1-n} \leq N-1} \beta_{k_{1}}^{-1} \cdots \beta_{k_{N-1-n}}^{-1}
$$

or, with the definition of the elementary symmetric functions, to

$$
\frac{1}{\beta_{1} \cdots \beta_{N-1}} S_{n}\left(\beta_{1}, \ldots, \beta_{N-1}\right)=S_{N-1-n}\left(\beta_{1}^{-1}, \ldots, \beta_{N-1}^{-1}\right) .
$$

Since the elementary symmetric functions are symmetric polynomials, we can permutate the arguments in arbitrary manner. Using that the complex zeros appear in conjugate pairs, we can rearrange the arguments after conjugating them on the right side such that the assertion follows.

For $n$ equal to 0 or $N-1$, the assertion follows directly from the definition of the elementary symmetric function since $S_{0}$ is always one, $S_{N-1}$ is the product of all arguments, and all complex numbers in $\left\{\beta_{1}, \ldots, \beta_{N-1}\right\}$ appear in complex pairs. For the remaining integers $n$ the equation is trivial because both sides are zero by definition.

Using this property of the elementary symmetric functions, we can now prove that the positivity constraints in Theorem 8.8 for the monic polynomial with the zero set $\left\{\beta_{1}, \ldots, \beta_{N-1}\right\}$ and for the monic polynomial with the reflected zero set at the unit circle are themselves reflected versions of each other.

Proposition 9.4. Let $\left\{\beta_{1}, \ldots, \beta_{N-1}\right\}$ be a multiset of non-zero complex numbers where the complex elements occur in conjugate pairs. Assume that $\beta_{N-2}$ and $\beta_{N-1}$ form a conjugate pair, and define

$$
\sigma_{n}:=(-1)^{n} S_{n}\left(\beta_{1}, \ldots, \beta_{N-3}\right) \quad \text { and } \rho_{n}:=(-1)^{n} S_{n}\left(\bar{\beta}_{1}^{-1}, \ldots, \bar{\beta}_{N-3}^{-1}\right)
$$

for every integer $n$. Then, for each integer $n$, the last element $\beta_{N-1}$ fulfils the inequality

$$
\sigma_{n-2}\left|\beta_{N-1}\right|^{2}-2 \sigma_{n-1} \Re \beta_{N-1}+\sigma_{n} \geq 0
$$

if and only if it also fulfils the inequality

$$
\operatorname{sgn}\left((-1)^{N-1} \beta_{1} \cdots \beta_{N-1}\right)\left(\rho_{N-3-n}\left|\bar{\beta}_{N-1}^{-1}\right|^{2}-2 \rho_{N-2-n} \mathfrak{R} \bar{\beta}_{N-1}^{-1}+\rho_{N-1-n}\right) \geq 0 .
$$


Proof. Except for the multiplication with $(-1)^{n}$, the constants $\sigma_{n}$ and $\rho_{n}$ are the elementary symmetric functions of the set $\left\{\beta_{1}, \ldots, \beta_{N-3}\right\}$ and its reflection at the unit circle. As a consequence of the identity for the elementary symmetric function in Lemma 9.3, the constants $\sigma_{n}$ and $\rho_{n}$ are hence related by

$$
\frac{1}{\beta_{1} \cdots \beta_{N-3}} \sigma_{n}=(-1)^{N-3} \rho_{N-3-n} .
$$

Using this equality after multiplication with $\beta_{1} \cdots \beta_{N-3}$, we can replace the constants $\sigma_{n}$ in the first inequality

$$
\sigma_{n-2}\left|\beta_{N-1}\right|^{2}-2 \sigma_{n-1} \Re \beta_{N-1}+\sigma_{n} \geq 0
$$

of the assertion, and we can write it in the equivalent form

$$
(-1)^{N-3} \beta_{1} \cdots \beta_{N-3}\left(\rho_{N-1-n}\left|\beta_{N-1}\right|^{2}-2 \rho_{N-2-n} \mathfrak{R} \beta_{N-1}+\rho_{N-3-n}\right) \geq 0 .
$$

In the next step, we factor out the missing $\beta_{N-2}$ and $\beta_{N-1}$ in the prefactor. Since $\beta_{N-2}$ and $\beta_{N-1}$ form a conjugate pair, we can use the equalities

$$
\beta_{N-2} \beta_{N-1}=\left|\beta_{N-1}\right|^{2} \quad \text { and } \quad \frac{\mathfrak{R} \beta_{N-1}}{\beta_{N-2} \beta_{N-1}}=\mathfrak{R} \bar{\beta}_{N-1}^{-1} \text {. }
$$

In this manner, the inequality above becomes

$$
(-1)^{N-3} \beta_{1} \cdots \beta_{N-1}\left(\rho_{N-1-n}-2 \rho_{N-2-n} \mathfrak{R} \bar{\beta}_{N-1}^{-1}+\rho_{N-3-n}\left|\bar{\beta}_{N-1}^{-1}\right|^{2}\right) \geq 0 .
$$

Rearranging the three terms in the sum, multiplying the inequality with $(-1)^{2}$, and dividing it by $\left|\beta_{1} \cdots \beta_{N-1}\right|$ leads to the assertion.

Remark 9.5. The reflection of the non-negativity conditions that we can observe in Figure 9.1 is hidden in Proposition 9.4. If we assume that the signal or the monic polynomial corresponding to the zero set $\left\{\beta_{1}, \ldots, \beta_{N-1}\right\}$ is non-negative or positive respectively, then the constant term

$$
(-1)^{N-1} S_{N-1}=(-1)^{N-1} \beta_{1} \cdots \beta_{N-1}
$$

given by VIETA's formulae (Theorem 8.7) is positive too. Hence, the sign of the prefactor in the second inequality in Proposition 9.4 is one and vanishes. Due to this fact, the last element $\beta_{N-1}$ fulfils the inequality

$$
\sigma_{n-2}\left|\beta_{N-1}\right|^{2}-2 \sigma_{n-1} \mathfrak{R} \beta_{N-1}+\sigma_{n} \geq 0
$$


if and only if it also fulfils the inequality

$$
\rho_{N-3-n}\left|\bar{\beta}_{N-1}^{-1}\right|^{2}-2 \rho_{N-2-n} \mathfrak{R} \bar{\beta}_{N-1}^{-1}+\rho_{N-1-n} \geq 0 ;
$$

so the sets of points that satisfy these conditions are indeed reflected versions of each other. Since this observation is valid for every integer $n$, the intersection of the half planes and circles for the zero set $\left\{\beta_{1}, \ldots, \beta_{N-3}\right\}$ and the reflected zero set are themselves reflections at the unit circle as conjectured.

The observations above can also be interpreted in a slightly different way. If we assume that the signal or monic polynomial to the zero set $\left\{\beta_{1}, \ldots, \beta_{N-1}\right\}$ is non-negative or positive respectively, then the last zero $\beta_{N-1}$ must satisfy the first inequality (9.1) for every integer $n$. Since the sign of the prefactor in the second inequality (9.2) is one as discussed above, the reflected last zero $\bar{\beta}_{N-1}^{-1}$ has to fulfil the second condition (9.2) for every integer $n$ too. Now, we can conclude that also the signal or monic polynomial with the reflected zero set $\left\{\bar{\beta}_{1}^{-1}, \ldots, \bar{\beta}_{N-1}^{-1}\right\}$ is non-negative or positive respectively. In some sense, this observation is trivial because the reflected zero set corresponds to the reflected signal in this specific case, see Lemma 5.3, and because the reflection of a non-negative signal is also clearly non-negative.

Remark 9.6. The cumbersome prefactor $\operatorname{sgn}\left((-1)^{N-1} \beta_{1} \cdots \beta_{N-1}\right)$ of the second condition in Proposition 9.4 is an artefact of the identification of a real-valued signal with a monic polynomial. More precisely, in Section 8, we have identified the non-trivial part

$$
\underset{j=1}{*} \underset{*}{*}\left(\delta_{1}-\beta_{j} \delta_{0}\right)
$$

in the characterization of a non-trivial solution (Theorem 5.1) with the monic polynomial

$$
\prod_{j=1}^{N-1}\left(z-\beta_{j}\right)
$$

and investigated the positivity of this polynomial. In Proposition 9.4, we have related the positivity condition on the last zero pair $\left(\beta_{N-2}, \beta_{N-1}\right)$ for the $n$th coefficient to the positivity condition on the reflected last zero pair $\left(\bar{\beta}_{N-2}^{-1}, \bar{\beta}_{N-1}^{-1}\right)$ for the $(N-1-n)$ th coefficient of the monic polynomial with the reflected zero set at the unit circle.

Now, we consider the reflection of the non-trivial part (9.3). Using Lemma 5.3 together with an additional shift to normalize the support of the signal and with 
an additional division by $\left|\beta_{1} \cdots \beta_{N-1}\right|$, we can write the reflection as

$$
\operatorname{sgn}\left((-1)^{N-1} \bar{\beta}_{1} \cdots \bar{\beta}_{N-1}\right) \cdot \mathscr{*}\left(\delta_{1}-\bar{\beta}_{j}^{-1} \delta_{0}\right) \text {. }
$$

The special case that the prefactor is equal to 1 and vanishes has been discussed in Remark 9.5. If we now assume that the prefactor is equal to -1 , we can conclude that the constant term of the monic polynomial (9.4) is negative. Moreover, except from a multiplicative positive constant, the reflected signal above possesses a negative leading coefficient. Hence, the monic polynomial to the reflected zero set has the negative coefficients of the reflected signal. This additional change of the sign of all coefficients explains the inversion of the second inequality in Proposition 9.4 to ensure the positivity of the $(N-1-n)$ th coefficient of the monic polynomial with the reflected zero set in a natural way.

\subsection{Continuity of the phase retrieval problem}

If we look back at Example 9.2, where we have extended a given zero set contained in the left half plane such that the corresponding phase retrieval problem possesses a certain number of non-negative non-trivial solutions, it seems that the non-negativity usually cannot reduce the number of arising non-trivial ambiguities. For an arbitrary given zero set, however, the situation can change dramatically as seen for instance in Example 8.10, where the intersection of the non-negativity constraints for one signal is already a small set. Although we cannot see the efficiency of the non-negativity constraint directly, we can nevertheless use our findings to show that neither uniqueness nor ambiguousness under the non-negativity constraint are rare exceptions.

By VIETA's formulae, the coefficients of a monic polynomial continuously depend on the zeros. Conversely, the zeros of a monic polynomial continuously depend on the coefficients as well, see for instance [Ort72, Theorem 3.1.1]. Further, this circumstance can be used to show that the non-trivial solutions of the phase retrieval problem continuously depend on their corresponding zero sets, and vice versa. In order to avoid the trivial shift ambiguity, we normalize the support of the considered signals. More precisely, a discrete-time signal $x$ has a normalized support of length $N$ if the support of $x$ is contained in $\{0, \ldots, N-1\}$ and, moreover, if $x[0]$ and $x[N-1]$ are non-zero.

Lemma 9.7. Let $x$ be a discrete-time signal with normalized support of length $N$ and corresponding zeros $\beta_{j}$. For every sufficiently small number $\varepsilon>0$, there exists a number $\delta>0$ such that the corresponding zeros $\breve{\beta}_{j}$ of every signal $\breve{x}$ with nor- 
malized support of length $N$ and $|\breve{x}[n]-x[n]| \leq \delta$ for $n$ from 0 to $N-1$ can be ordered in such a way that

$$
\left|\breve{\beta}_{j}-\beta_{j}\right| \leq \varepsilon
$$

for $j$ from 0 to $N-1$.

Proof. Based on the real-valued or complex-valued signal $x$ with normalized support of length $N$ and corresponding zero set $\left\{\beta_{1}, \ldots, \beta_{N-1}\right\}$, we consider the monic polynomial

$$
P(z)=\frac{1}{x[N-1]} \sum_{n=0}^{N-1} x[n] z^{n}
$$

with the same zero set $\left\{\beta_{1}, \ldots, \beta_{N-1}\right\}$ as discussed in Section 8. In the following, we denote the coefficients of the monic polynomial $P$ by $c_{n}:=x[n] / x[N-1]$. Using the continuity of roots theorem, see [Ort72, Theorem 3.1.1], we find, for every sufficiently small number $\varepsilon>0$, a number $\eta>0$ such that the zeros $\breve{\beta}_{j}$ of all monic polynomials

$$
Q(z):=z^{N-1}+\breve{c}_{N-2} z^{N-2}+\cdots+\breve{c}_{0}
$$

with $\left|\breve{c}_{n}-c_{n}\right| \leq \eta$ for $n$ from 0 to $N-2$ can be ordered in a way that

$$
\left|\breve{\beta}_{j}-\beta_{j}\right| \leq \varepsilon
$$

for $j$ from 1 to $N-1$.

Furthermore, if we consider the discrete-time signal $x$ with normalized support of length $N$ as an $N$-dimensional vector, then the mapping between the components $x[n]$ of the signal and the coefficients $c_{n}$ of the monic polynomial $P$ given by

$$
(x[0], \ldots, x[N-1]) \mapsto\left(\frac{x[0]}{x[N-1]}, \ldots, \frac{x[N-2]}{x[N-1]}\right)
$$

is obviously continuous. Hence, for every sufficiently small number $\eta>0$, there exists a number $\delta>0$ such that the components of the image of every vector $\breve{x}$ in $\mathbb{R}^{N}$ or in $\mathbb{C}^{N}$ with $|\breve{x}[n]-x[n]| \leq \delta$ for $n$ from 0 to $N-1$ satisfy

$$
\left|\frac{\breve{x}[n]}{\breve{x}[N-1]}-\frac{x[n]}{x[N-1]}\right| \leq \eta \quad \text { or } \quad\left|\breve{c}_{n}-c_{n}\right| \leq \eta
$$

for $n$ from 0 to $N-2$. In order to avoid that $\breve{x}[N-1]$ becomes zero, we assume without loss of generality that $\delta<x[N-1]$. Interpreting the vector $\breve{x}$ as discrete-time signal with normalized support of length $N$ and combining both constructions yield the assertion. 
Remark 9.8. If we consider the discrete-time signals $\breve{x}$ Lemma 9.7 as $N$-dimensional vectors, these signals form a closed ball with respect to the maximum norm. Moreover, we can extend this ball to a cone since the multiplication of a signal with a positive real constant does not change the corresponding zero set. By construction, the resulting cone cannot be contained in a set with zero Lebesgue measure. Consequently, this cone is an unbounded set with infinite measure.

Using the continuity relation between the discrete-time signal coefficients and the corresponding zero set, we will show that the behaviour of the non-trivial solution set does not change in a small neighbourhood of the signal with respect to the maximum norm.

For example, if we consider a signal $x$ whose corresponding zeros have a negative real part, then the signal $x$ itself and all arising non-trivial ambiguities are non-negative as stated in Proposition 9.1. Assuming that the signal $x$ has a normalized support, and using Lemma 9.7, we can obviously find a $\delta$-neighbourhood of $x$ such that the corresponding zero sets of the signals in this $\delta$-neighbourhood are completely contained in the left half plane. Thus, the phase retrieval of these signals only provides non-negative non-trivial ambiguities too. Hence, the behaviour of the non-trivial solution set does not change in a small neighbourhood around the original signal $x$.

If we assume that the signal $x$ with normalized support of length $N$ additionally possesses only positive components $x[n]$ for $n$ from 0 to $N-1$, and that all non-trivial solutions of the phase retrieval problem to recover the signal $x$ also satisfy this assumption, then we can generalize the above observation.

Theorem 9.9. Let $x$ be a discrete-time signal with normalized support of length $N$ and positive components $x[n]$ for $n$ from 0 to $N-1$. If all non-trivial ambiguities of the phase retrieval problem to recover $x$ satisfy the same assumptions, then there exists a number $\delta>0$ so that all non-trivial ambiguities of the phase retrieval problem to recover a signal $\breve{x}$ with normalized support of length $N$ and $\mid \breve{x}[n]-$ $x[n] \mid \leq \delta$ for $n$ from 0 to $N-1$ possess only positive components over the support $\{0, \ldots, N-1\}$.

Proof. Due to the fact that the discrete-time signal $x$ with normalized support possesses only positive components $x[n]$ for $n$ from 0 to $N-1$, the corresponding zero set $\Lambda:=\left\{\beta_{1}, \ldots, \beta_{N-1}\right\}$ fulfils the non-negativity conditions

$$
\sigma_{n-2}\left|\beta_{N-1}\right|^{2}-2 \sigma_{n-1} \mathfrak{R} \beta_{N-1}+\sigma_{n} \geq 0
$$


in Theorem 8.8 strictly. The left-hand side of this inequality can be interpreted as a continuous function of the variables $\beta_{1}, \ldots, \beta_{N-1}$ since the elementary symmetric functions in $\sigma_{n}:=(-1)^{n} S_{n}\left(\beta_{1}, \ldots, \beta_{N-3}\right)$ are obviously continuous. Hence, we find a small $\varepsilon$-ball with respect to the maximum norm and with $\varepsilon>0$ around $\Lambda$ such that every zero set $M:=\left\{\breve{\beta}_{1}, \ldots, \breve{\beta}_{N-1}\right\}$ with $\left|\breve{\beta}_{j}-\beta_{j}\right| \leq \varepsilon$ for $j$ from 1 to $N-1$ satisfies the non-negativity constraints strictly too.

Now, we consider the non-negativity conditions for the non-trivial ambiguities analogously. As described in Example 9.2, we obtain the non-negativity conditions for a certain ambiguity by reflecting some zeros in $\Lambda$ at the unit circle. The non-negativity constraints for a certain non-trivial ambiguity can therefore be written as a set of inequality conditions in analogy to Theorem 8.8. Since the reflection at the unit circle is a continuous map, and since the elements in $\Lambda$ cannot be zero, the left-hand sides of this inequality conditions depend continuously on the zeros in $\Lambda$. Hence, for all non-trivial ambiguities, we find a small ball around $\Lambda$ so that the zero sets in this neighbourhood fulfil the corresponding non-negativity conditions strictly. Taking the smallest of the finitely many constructed neighbourhoods and applying Lemma 9.7 yields the assertion.

Remark 9.10. In Theorem 9.9, the number of non-trivial ambiguities of the phase retrieval problems to recover a signal in the constructed neighbourhood do not have to be equal and can change dramatically. For example, if the zero set $\left\{\beta_{1}, \ldots, \beta_{N-1}\right\}$ of the original signal $x$ lies on the unit circle, then the signal $x$ can be recovered without any non-trivial ambiguities, see Proposition 6.1. However, most of the corresponding zero sets of the signals $\breve{x}$ in a $\delta$-neighbourhood of $x$ will not completely lie on the unit circle, and the non-trivial ambiguities in Theorem 5.1 will not coincide.

In order to overcome the problem of the changing number of non-trivial ambiguities, we consider only discrete-time signals whose corresponding zeros $\beta_{j}$ are pairwise different, do not appear in reflected pairs $\left(\beta_{j}, \bar{\beta}_{j}^{-1}\right)$, and do not lie on the unit circle. Then the solutions characterized in Theorem 5.1 are pairwise different except for the trivial reflection ambiguity. Adapting the proof of Theorem 9.9, we can show the following two corollaries for the discrete-time phase retrieval problem of non-negative real-valued signals.

Corollary 9.11. Let $x$ be a discrete-time signal with normalized support of length $N$, positive components $x[n]$ for $n$ from 0 to $N-1$, and pairwise different zeros $\beta_{j}$ not appearing in reflected pairs and not lying on the unit circle. If the phase retrieval problem to recover the signal $x$ possesses at least a further non-trivial ambiguity 
satisfying the same assumptions, then there exists a number $\delta>0$ so that every signal $\breve{x}$ with normalized support of length $N$ and $|\breve{x}[n]-x[n]| \leq \delta$ for $n$ from 0 to $N-1$ cannot be recovered uniquely by using the non-negativity constraint.

Proof. Instead of the non-negativity constraints for all non-trivial ambiguities, here we consider only the non-negativity constraints of the original signal $x$ with zero set $\Lambda:=\left\{\beta_{1}, \ldots, \beta_{N-1}\right\}$ and of one further non-trivial solution. Since we assume that the signal $x$ and the further ambiguity possesses positive components over the support $\{0, \ldots, N-1\}$, the zeros in $\Lambda$ fulfil the non-negativity conditions in Theorem 8.8 strictly. Following the proof of Theorem 9.9, we find a small $\varepsilon$-ball with respect to the maximum norm and with $\varepsilon>0$ around $\Lambda$ so that the zero sets in this neighbourhood satisfy both non-negativity constraints strictly too.

Keeping in mind that the corresponding zeros $\beta_{j}$ in $\Lambda$ are pairwise different and do not lie on the unit circle, we can shrink the $\varepsilon$-ball so that the $2 N-2$ discs around the single zeros $\beta_{j}$ and their reflections $\bar{\beta}_{j}^{-1}$ do not intersect in the complex plane and do not contain points from the unit circle. In this manner, the different solutions in Theorem 5.1 cannot coincide. Using Lemma 9.7 now yields the assertion in analogy to the proof of Theorem 9.9.

Corollary 9.12. Let $x$ be a discrete-time signal with normalized support of length $N$, positive components $x[n]$ for $n$ from 0 to $N-1$, and pairwise different zeros $\beta_{j}$ not appearing in reflected pairs and not lying on the unit circle. If the phase retrieval problem to recover the signal $x$ is uniquely solvable up to reflection, then there exists a number $\delta>0$ so that every signal $\breve{x}$ with normalized support of length $N$ and $|\breve{x}[n]-x[n]| \leq \delta$ for $n$ from 0 to $N-1$ can be recovered uniquely up to reflection.

Proof. Instead of the non-negativity constraints for all non-trivial ambiguities, here we consider only the non-negativity constraints of the original signal $x$ with zero set $\Lambda:=\left\{\beta_{1}, \ldots, \beta_{N-1}\right\}$. As in the proof of Theorem 9.9, the zeros in $\Lambda$ satisfy these constraints strictly. Since the phase retrieval problem to recover the signal $x$ from its Fourier intensity is uniquely solvable, the further nontrivial ambiguities do not fulfil at least one of the corresponding non-negativity constraints in Theorem 8.8.

Using the continuity of the these conditions, we find a small $\varepsilon$-ball with respect to the maximum norm around $\Lambda$ so that the zero sets in this neighbourhood also satisfy the non-negativity constraints strictly and that the further zero sets that can be constructed by reflecting zeros at the unit circle also do not fulfil 
at least one of the corresponding non-negativity constraints. Shrinking the $\varepsilon^{-}$ ball as discussed in the proof of Corollary 9.11 and using Lemma 9.7 lead to the assertion analogously to the proof of Theorem 9.9.

Although we need some additional assumptions on the considered discretetime signal in Corollary 9.11 and Corollary 9.12, we can find such signals for every support length $N$. A real-valued discrete-time signal that satisfies the assumptions of Corollary 9.11 can be constructed straightforwardly by using our findings about HuRwitzian polynomials and their corresponding signals.

Proposition 9.13. For every integer $N>3$, there exists a discrete-time signal $x$ with normalized support of length $N$, positive components $x[n]$ for $n$ from 0 to $N-1$, and pairwise different zeros $\beta_{j}$ not appearing in reflected pairs and not lying on the unit circle such that the phase retrieval problem to recover the signal $x$ possesses at least a further non-trivial ambiguity satisfying the same assumptions.

Proof. In order to construct a real-valued discrete-time signal $x$ that satisfies the required assumptions, we start with an appropriately selected corresponding zero set $\Lambda:=\left\{\beta_{1}, \ldots, \beta_{N-1}\right\}$. More precisely, we assume that our zero set $\Lambda$ consists of pairwise different zeros which do not appear in reflected pairs, do not lie on the unit circle, and have a purely negative real part. Furthermore, we can choose the zeros $\beta_{j}$ in a way so that our zero set $\Lambda$ contains more than one real or one conjugate zero pair since the cardinality of the corresponding zero set is $N-1$ and hence at least two.

Defining the discrete-time signal $x$ with the corresponding zero set $\Lambda$ by

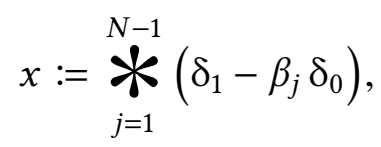

we immediately see that the signal $x$ has a normalized support of length $N$. Furthermore, since the real part of all zeros $\beta_{j}$ is negative, we can conclude that the components $x[n]$ for $n$ from 0 to $N-1$ are positive as already discussed in the proof of Corollary 8.11. Consequently, our signal $x$ fulfils all requirements of Proposition 9.13.

It remains to prove that the phase retrieval problem to recover the constructed signal $x$ possesses at least one further non-trivial ambiguity that satisfies the required assumptions. To begin with, Proposition 6.1 and Remark 6.2 imply that the considered phase retrieval problem indeed has several non-trivially different 
solutions because the corresponding zero set $\Lambda$ contains more than one real zero or one conjugate zero pair.

Since we only have selected corresponding zeros with negative real part, all occurring non-trivial ambiguities are non-negative signals by Proposition 9.1. If we normalize the support of the non-trivial ambiguities to $\{0, \ldots, N-1\}$ with an additional shift and utilize that the real part of the reflections $\bar{\beta}_{j}^{-1}$ is also negative, we can observe that the non-trivial ambiguities $y$ also possess positive components $y[n]$ for $n$ from 0 to $N-1$. Consequently, all arising non-trivial ambiguities also satisfy the requirements of Proposition 9.13.

Next, we want to find a real-valued discrete-time signal that fulfils the assumptions of Corollary 9.12. Similarly as in the proof of Proposition 9.13, we can explicitly construct such a signal by selecting the corresponding zero set appropriately. To ensure the uniqueness of the phase retrieval problem, we can especially use Corollary 8.11 and our observations in Example 8.12.

Proposition 9.14. For every integer $N$, there exists a discrete-time signal $x$ with normalized support of length $N$, positive components $x[n]$ for $n$ from 0 to $N-1$, and pairwise different zeros $\beta_{j}$ not appearing in reflected pairs and not lying on the unit circle such that the phase retrieval problem to recover the signal $x$ is uniquely solvable up to reflection by using the non-negativity constraint.

Proof. To begin with, the assertion holds obviously true for a support length $N$ from one to three. More detailed, we can easily construct a discrete-time signal $x$ such that the corresponding zero set contains only one real zero or one complex zero pair or is completely empty. Now, the phase retrieval problem to recover such a signal is uniquely solvable due to the simple fact that no other non-trivial ambiguities can arise because the solution with the reflected zero set is simply the reflection of the original signal $x$ and hence trivial, see Lemma 5.3.

For support lengths $N>3$, we again try to construct the signal $x$ fulfilling the requirements by selecting the corresponding zero set appropriately. However, since the phase retrieval problem to recover the signal $x$ shall be uniquely solvable up to rotation, the explicite choice of the corresponding zeros is crucial. Here we use a similar approach as in Example 8.12 and Example 9.2, this is, beginning with a fixed zero set $\left\{\beta_{1}, \ldots, \beta_{N-3}\right\}$ of real zeros and conjugate zero pairs, we try to extend this set by a further conjugate zero pair so that all components 
of the discrete-time signal

$$
x=\underset{j=1}{\mathbb{K}}\left(\delta_{1}-\beta_{j} \delta_{0}\right)
$$

are non-negative, and that each further non-trivial solution possesses at least one negative component. We use the slightly simpler non-negativity conditions in Corollary 8.11 and start from a zero set $\left\{\beta_{1}, \ldots, \beta_{N-3}\right\}$ whose pairwise different zeros do not appear in reflected pairs, do not lie on the unit circle, and, moreover, have a real part less than -1 .

Next, we consider the non-negativity restrictions in Corollary 8.11 for our specific zero set. To ensure the non-negativity of the constructed signal $x$ itself, the last conjugate zero pair has to lie in the half plane left of the imaginary axis through $\sigma_{1} / 2$. Using the definition of $\sigma_{n}$ in Corollary 8.11 and the assumption that the fixed zero set consists of real zeros and conjugate zero pairs, this condition means nothing but

$$
\mathfrak{R} \beta_{N-1} \leq-\frac{1}{2}\left(\mathfrak{R} \beta_{1}+\cdots+\mathfrak{R} \beta_{N-3}\right) .
$$

As discussed in Example 9.2, the corresponding non-negativity restrictions of the remaining non-trivial ambiguities have the same form and can be obtained by replacing some zeros $\beta_{j}$ by their reflections at the unit circle.

However, since the real part of the fixed zeros $\beta_{j}$ is less than -1 , and hence the real part of the reflected zeros is greater than -1 , the replacement of some zeros by their reflections leads to a strictly smaller right-hand side of (9.5). Consequently, we can choose the last conjugate zero pair $\left(\beta_{N-2}, \beta_{N-1}\right)$ in a way such that the extended zero set $\Lambda:=\left\{\beta_{1}, \ldots, \beta_{N-1}\right\}$ satisfies the non-negativity condition in (9.5) strictly, and, moreover, the corresponding zero sets of the further non-trivial ambiguities violate this condition. Figuratively, the real part of the last zero $\beta_{N-1}$ has to lie in an appropriate bounded open interval, or the last zero pair must be chosen from an appropriately small band in the complex plane, $\mathrm{cf}$. Figure 9.1(a).

If the constructed zero set $\Lambda$ also satisfies the remaining non-negativity constraints, then the phase retrieval problem to recover the signal $x$ has a unique non-negative solution up to reflection. Since we can use the simplified restrictions in Corollary 8.11, the last conjugate zero pair must lie on or outside the circles with centre $\sigma_{n-1} / \sigma_{n-2}$ and radius $\sqrt{\sigma_{n-1}^{2}-\sigma_{n} \sigma_{n-2}} / \sigma_{n-2}$ for $n$ from 2 to $N-2$ whenever the radius is real. Heretofore, the imaginary part of the last zero $\beta_{N-1}$ can be chosen arbitrarily; consequently, we always find a suitable last conjugate zero pair that strictly lies outside the circles in Corollary 8.11. 
If we look back at the proof of Theorem 8.8, we observe that a coefficient of the considered monic polynomial can be zero if and only if the corresponding nonnegativity condition holds with equality. Bearing in mind that the constructed zero set $\Lambda$ satisfies the non-negativity conditions in Corollary 8.11 and hence in Theorem 8.8 strictly, we can conclude that the components $x[n]$ for $n$ from 0 to $N-1$ are positive. So all in all, the constructed signal $x$ fulfils the assumptions, and the assertion follows.

If we combine our findings in this section, we can finally show that neither the uniqueness nor the ambiguousness under the non-negativity constraint is a rare exception. Hence, the assumed non-negativity of a discrete-time signal can be used to enforce the uniqueness of the corresponding phase retrieval problem, but unfortunately not for every signal.

Theorem 9.15. If the real-valued discrete-time signals with normalized support of length $N$ with $N>3$ are considered as real $N$-dimensional vectors, then the set of signals that can be recovered uniquely up to reflection as well as the set of signals that cannot be recovered uniquely from their FOURIER intensities together with the non-negativity constraint are unbounded sets containing a cone of infinite LEBESGUE measure.

Proof. Using Proposition 9.14, we find at least one signal $x$ that can be recovered uniquely from its Fourier intensity up to reflection. Moreover, this signal fulfils the assumptions of Corollary 9.12; so we find a sufficient small $\delta>0$ such that every signal $\breve{x}$ with normalized support of length $N$ and $|\breve{x}[n]-x[n]| \leq \delta$ for $n$ from 0 to $N-1$ can be recovered uniquely up to reflection. As discussed in Remark 9.8, the signals $\breve{x}$ form a closed ball with respect to the maximum norm in $\mathbb{R}^{N}$, and this ball can be extended to a unbounded cone with infinite LEBESGUE measure. Hence, the set of signals that can be recovered uniquely up to reflection is unbounded and contains a cone with infinite measure, as conjectured.

Replacing Proposition 9.14 by Proposition 9.13 and Corollary 9.12 by Corollary 9.11, we can analogously prove the assertion for the set of signals that cannot be recovered uniquely. 
Chapter III.

\title{
Additional information in the time domain
}

\begin{abstract}
Besides the Fourier intensity in the frequency domain, we will now assume that we have access to additional data about the unknown signal in the time domain. Restricting themselves to signals with real-valued components, $\mathrm{XU}$, YAN, and CHANG [XYC87] have shown that almost all signals can be uniquely recovered if one of the end points in the time domain is known beforehand. Using our characterization of the solutions in the time domain, we will obtain a new approach to this specific phase retrieval problem and will be able to generalize the ideas of Xu et al. to complex-valued signals. Based on this observations, we will more generally investigate the ability of moduli, phases, and signal values of arbitrary points in the time domain to enforce uniqueness. Finally, we will give a series of novel and complete proofs in order to show that additional information of this nature can really ensure a unique recovery of the unknown signal almost always.

${ }^{a}$ Some of the observations in this chapter are briefly mentioned in [BP15a].

\section{Using additional magnitudes of the unknown signal}

A completely different idea to enforce uniqueness of the discrete-time phase retrieval problem is to use additional measured data of the unknown signal in the time domain instead of an a priori condition like the non-negativity. One simple idea dates back to XU, YAN, and CHANG in [XYC87]. Using the additionally known end point $x[N-1]$ of a real-valued discrete-time signal $x:=(x[n])_{n \in \mathbb{Z}}$ with normalized support $\{0, \ldots, N-1\}$ of length $N$, XU et al. prove that almost every signal can be uniquely recovered from its FoURIER intensity without any trivial ambiguities. In the following, we generalize this idea to the complex case, which can be done straightforwardly. Moreover, we examine how far other additionally known signal values can enforce uniqueness. 
The central idea in the proof of Xu et al. is to show that the signals which cannot be uniquely recovered from their FourIER intensities and the additional end point form a set with zero LEBESGUE measure. In other words, we have to determine all signals such that at least one further non-trivial solution possesses the same end point. However, since we can always add a global phase to a complexvalued solution without changing the FourIER intensity, see Proposition 2.1, the corresponding question in the complex case is: can we find a further non-trivial solution whose end point has the same absolute value as the last non-zero component of the original signal, or asked differently, can we recover a signal with normalized support from its FOURIER intensity and the modulus of its end point?

\subsection{The modulus of the end point}

Before we show that almost every complex-valued signal can be uniquely recovered from its Fourier intensity and the modulus of its end point, we investigate whether the corresponding phase retrieval problem possesses at least one further non-trivial solution. Since the Fourier intensity $|\widehat{x}|$ of a discretetime signal $x$ with finite support is a non-negative trigonometric polynomial and is completely determined by $2 N-1$ samples at different points in $[-\pi, \pi)$, see Remark 3.5, we once more assume that the complete FourIER intensity $|\widehat{x}|$ is known. In the following theorem, we will see that the uniqueness of the considered phase retrieval problem directly depends on the corresponding zeros $\beta_{j}$ within the characterization in Theorem 5.1.

Theorem 10.1. Let $x$ be a complex-valued discrete-time signal with normalized support of length $N$ and corresponding zero set $B:=\left\{\beta_{1}, \ldots, \beta_{N-1}\right\}$. Then the signal $x$ can be uniquely recovered from its FOURIER intensity $|\widehat{x}|$ and the modulus of its end point $|x[N-1]|$ up to rotations if and only if the corresponding zeros $\beta_{j}$ fulfil

$$
\prod_{\beta_{j} \in \Lambda}\left|\beta_{j}\right| \neq 1
$$

for each non-empty subset $\Lambda$ of $B$ where $\Lambda$ does not contain reflected zero pairs or zeros on the unit circle.

Proof. Adapting the characterization of all discrete-time signals with the same FoURIER intensity in Theorem 5.1 to the normalized support, we can write the 
original signal $x$ in the form

$$
x=\mathrm{e}^{\mathrm{i} \alpha} \sqrt{|a[N-1]| \prod_{j=1}^{N-1}\left|\beta_{j}\right|^{-1}} \cdot\left[\underset{j=1}{\mathbb{*}}\left(\delta_{1}-\beta_{j} \delta_{0}\right)\right],
$$

where $\alpha$ is a suitable real number and $a$ is the autocorrelation signal of $x$. Further, the expansion of the convolution yields that the modulus of the end point can be written as

$$
|x[N-1]|=\sqrt{|a[N-1]| \prod_{j=1}^{N-1}\left|\beta_{j}\right|^{-1}} .
$$

Now, we assume that the phase retrieval problem to recover the signal $x$ has at least one further solution $\breve{x}$ with corresponding zeros $\breve{\beta}_{j}$ where $\breve{x}$ is no rotation of the original signal. The modulus of the end point of the second solution $\breve{x}$ is analogously given by

$$
|\breve{x}[N-1]|=\sqrt{|a[N-1]| \prod_{j=1}^{N-1}\left|\breve{\beta}_{j}\right|^{-1}} .
$$

In order to simplify the notation in the following, we assume according to the proof of Theorem 5.5 that the corresponding zero sets of $x$ and $\breve{x}$ are arranged in a way that

$$
\breve{\beta}_{j}:= \begin{cases}\bar{\beta}_{j}^{-1} & j=1, \ldots, J, \\ \beta_{j} & j=J+1, \ldots, N-1\end{cases}
$$

for a natural number $J \in\{1, \ldots, N-1\}$. Since the corresponding zero sets of the original signal $x$ and the further non-trivial solution $\breve{x}$ are different, we can choose $J$ such that the set $\left\{\breve{\beta}_{1}, \ldots, \breve{\beta}_{J}\right\}$ is non-empty and does not contain reflected zero pairs or zeros on the unit circle.

Due to the assumption that the moduli of the end points of both signals $x$ and $\breve{x}$ are equal, the corresponding zero sets obviously have to satisfy

$$
\sqrt{|a[N-1]| \prod_{j=1}^{N-1}\left|\beta_{j}\right|^{-1}}=\sqrt{|a[N-1]| \prod_{j=1}^{N-1}\left|\breve{\beta}_{j}\right|^{-1}} .
$$

After canceling the prefactor and the equal zeros on both sides, we finally have 
the equation

$$
\prod_{j=1}^{J}\left|\beta_{j}\right|^{-\frac{1}{2}}=\prod_{j=1}^{J}\left|\bar{\beta}_{j}\right|^{\frac{1}{2}} \text { or } \prod_{j=1}^{J}\left|\beta_{j}\right|=1 \text {. }
$$

By our assumptions, the product over the first $J$ zeros does not contain reflected zero pairs or zeros on the unit circle.

Consequently, the phase retrieval problem to recover the signal $x$ from its FOURIER intensity and the modulus of its end point possesses further non-trivial solutions if and only if there exists a certain subset $\Lambda$ of the corresponding zero set whose elements do not arise in reflected pairs or lie on the unit circle such that the product over this zeros is unimodular. Negating this observation leads to the assertion.

Remark 10.2. The statement of Theorem 10.1 can be interpreted as a straightforward generalization of [XYC87, Theorem 1] to the complex case. Although the conditions to ensure a unique reconstruction of the unknown signal are similar, they arise from completely different a priori conditions and observations. The main difference here is that $\mathrm{Xu}$ et al. consider real-valued signals and assume that the complete end point $x[N-1]$ is known and not only the absolute value $|x[N-1]|$.

The proof of Theorem 10.1 for complex-valued signals is mainly based on our characterization of every solution - trivial or non-trivial - in Theorem 5.1, and the uniqueness condition directly follows from this characterization. Xu et al. use an entirely different approach. More detailed, the proof of [XYC87, Theorem 1] is based on the observation that the right end point $x[N-1]$ together with the corresponding zeros $\beta_{j}$ uniquely determines the left end point $x[0]$ by

$$
x[0]=x[N-1] \prod_{j=1}^{N-1}\left(-\beta_{j}\right) .
$$

A consideration of the autocorrelation function $\widehat{a}$ for two non-trivially different solutions $x$ and $\breve{x}$ under the assumption that $x[N-1]$ and $\breve{x}[N-1]$ coincide leads to the wanted uniqueness condition for the reconstruction of the real-valued signal $x$ from its FourIER intensity $|\widehat{x}|$ and its end point $x[N-1]$.

Remark 10.3. The statement of Theorem 10.1 remains valid if we assume that the left end point $|x[0]|$ is given instead of the right end point $|x[N-1]|$. Here a comparison of the absolute values $|x[0]|$ and $|\breve{x}[0]|$ of two non-trivially different 
solutions $x$ and $\breve{x}$ leads to the identity

$$
\sqrt{|a[N-1]| \prod_{j=1}^{N-1}\left|\beta_{j}\right|^{-1}} \prod_{j=1}^{N-1}\left|\beta_{j}\right|=\sqrt{|a[N-1]| \prod_{j=1}^{N-1}\left|\breve{\beta}_{j}\right|^{-1}} \prod_{j=1}^{N-1}\left|\breve{\beta}_{j}\right|,
$$

which yields the slightly changed assertion.

Next, we investigate which zero sets $\left\{\beta_{1}, \ldots, \beta_{N-1}\right\}$ do not fulfil the uniqueness conditions in Theorem 10.1. In order to describe these zero sets, we identify $\left\{\beta_{1}, \ldots, \beta_{N-1}\right\}$ with the real $(2 N-2)$-dimensional vector

$$
\left(\mathfrak{R} \beta_{1}, \mathfrak{J} \beta_{1}, \ldots, \mathfrak{R} \beta_{N-1}, \mathfrak{J} \beta_{N-1}\right)^{\mathrm{T}}
$$

and show that the vectors violating the uniqueness conditions are contained in the union of certain real manifolds of minor dimension, cf. [XYC87, p. 2099] for the real case.

Lemma 10.4. If we identify the set of all possible zero sets with $\left(\mathbb{R}^{2} \backslash\{0\}\right)^{N-1}$, then the zero sets satisfying

$$
\prod_{\beta_{j} \in \Lambda}\left|\beta_{j}\right|=1
$$

for a certain non-empty subset $\Lambda$ of the zeros $\beta_{j}$ form a real $(2 N-3)$-dimensional submanifold.

Proof. Remembering that the corresponding zeros $\beta_{j}$ in Theorem 5.1 are nonzero, we can identify each zero set $\left\{\beta_{1}, \ldots, \beta_{N-1}\right\}$ with a real $(2 N-2)$-dimensional vector

$$
\boldsymbol{\beta}:=\left(\mathfrak{R} \beta_{1}, \mathfrak{J} \beta_{1}, \ldots, \mathfrak{R} \beta_{N-1}, \mathfrak{J} \beta_{N-1}\right)^{\mathrm{T}} \in\left(\mathbb{R}^{2} \backslash\{0\}\right)^{N-1} .
$$

Again, to simplify the notation in the following, we assume that the subset $\Lambda$ is of the form $\Lambda:=\left\{\beta_{1}, \ldots, \beta_{J}\right\}$ for a natural number $J \in\{1, \ldots, N-1\}$.

If we consider a real vector $\boldsymbol{\beta}$ fulfilling the condition in Lemma 10.4 for a certain subset $\Lambda$, then the real part $\mathfrak{R} \beta_{1}$ can be reconstructed from the imaginary part $\mathfrak{J} \beta_{1}$ and the remaining components $\mathfrak{R} \beta_{j}$ and $\mathfrak{J} \beta_{j}$ for $j$ from 2 to $J$ except for the sign by

$$
\left[\mathfrak{R} \beta_{1}\right]^{2}=\prod_{j=2}^{J}\left|\beta_{j}\right|^{-2}-\left[\mathfrak{J} \beta_{1}\right]^{2},
$$

where the product is set to one for $J=1$. 
This circumstance can be used to define a set of appropriate charts and to show that the vectors $\boldsymbol{\beta}$ satisfying the condition (10.1) form a real smooth manifold. In the following, we exemplarily assume that $\mathfrak{R} \beta_{1}$ is positive, and define the first chart $\kappa:\left(\mathbb{R}^{2} \backslash\{0\}\right)^{N-1} \rightarrow \mathbb{R}^{2 N-3}$ by

$$
\kappa(\boldsymbol{\beta}):=\left(\mathfrak{J} \beta_{1}, \mathfrak{R} \beta_{2}, \ldots, \mathfrak{R} \beta_{N-1}, \mathfrak{J} \beta_{N-1}\right)^{\mathrm{T}} .
$$

With our observations above, the inverse chart $\kappa^{-1}$ is given by

$$
\kappa^{-1}(\cdot)=\left(\sqrt{\prod_{j=2}^{L}\left(\left[\mathfrak{R} \beta_{j}\right]^{2}+\left[\mathfrak{J} \beta_{j}\right]^{2}\right)^{-1}-\left[\mathfrak{J} \beta_{1}\right]^{2}}, \mathfrak{J} \beta_{1}, \mathfrak{R} \beta_{2}, \ldots, \mathfrak{J} \beta_{N-1}\right)^{\mathrm{T}}
$$

Obviously, the mappings $\kappa$ and $\kappa^{-1}$ are continuous, which implies that our chart $\kappa$ is a homeomorphism. Moreover, the rank of the JAcoBian $\boldsymbol{J}_{\kappa^{-1}}$ of the inverse mapping is $2 N-3$. Analogously, we can obtain a further chart for $\mathfrak{R} \beta_{1}<0$. For the remaining case that $\mathfrak{R} \beta_{1}$ is equal to zero, the required charts can be constructed in a similar way by considering the cases $\mathfrak{J} \beta_{1}>0$ and $\mathfrak{J} \beta_{1}<0$.

All in all, for every vector $\boldsymbol{\beta}$ fulfilling the condition of Lemma 10.4, there exists an open neighbourhood that can be mapped by one of the constructed homeomorphisms to an open subset in $\mathbb{R}^{2 N-3}$. Since the rank of the JACoBian of the inverse mappings is $2 \mathrm{~N}-3$ for all constructed charts, the vectors satisfying the conditions indeed form a real $(2 N-3)$-dimensional submanifold, see [SS62, Section 18.1] or [BF96, Section 17.5], for instance.

Applying the construction in Lemma 10.4 to every possible subset $\Lambda$, we know that the corresponding zero sets of the signals that do not satisfy the uniqueness conditions in Theorem 10.1 are contained in the union of real $(2 \mathrm{~N}-3)$ dimensional submanifolds. Due to the reason that constructed submanifolds of minor dimension are sets with zero LEBESGUE measure in $\mathbb{R}^{2 N-2}$, the exceptional zero sets form a set of LEBESGUE measure zero. In order to transfer this observation to the coefficients of the corresponding signals with normalized support, we use the following variant of SARD's theorem stated in [Sch69, Theorem 3.1.].

Theorem 10.5 (SARD). Let $F: D \rightarrow \mathbb{R}^{n}$ be a continuously differentiable mapping where $D$ is an open set in $\mathbb{R}^{n}$. Then the image $F(E)$ of every measurable set $E \subset D$ is measurable, and the LEBESGUE measure $\lambda$ of the image $F(E)$ is bounded by

$$
\lambda(F(E)) \leq \int_{E}\left|\operatorname{det} \boldsymbol{J}_{F}(y)\right| \mathrm{d} y
$$

where $\boldsymbol{J}_{F}$ is the fACOBIan of F. 
Using SARD's theorem, we can finally prove that almost every signal with normalized support can be uniquely recovered from its FourIER intensity and the modulus of its end point up to rotations.

Theorem 10.6. If the complex-valued discrete-time signal $x$ with normalized support of length $N$ is considered as a real $2 N$-dimensional vector, then the phase retrieval problem to recover the signal $x$ from its FOURIER intensity $|\widehat{x}|$ and the $a b$ solute value $|x[N-1]|$ of its end point is almost always uniquely solvable up to rotations.

Proof. Starting from our findings in Lemma 10.4, where we observe that the corresponding zero sets $\left\{\beta_{1}, \ldots, \beta_{N-1}\right\}$ satisfying

$$
\prod_{\beta_{j} \in \Lambda}\left|\beta_{j}\right|=1
$$

for a certain subset $\Lambda$ of zeros $\beta_{j}$ form a real $(2 N-3)$-dimensional manifold, we can conclude that the exceptional zero sets which do not fulfil the uniqueness condition in Theorem 10.1 are contained in the union of finitely many real manifolds of minor dimension. Due to the fact that the conditions of Lemma 10.4 are slightly weaker as in Theorem 10.1 (the zeros can also occur in reflected pairs and can lie on the unit circle), the exceptional zero sets are subsets of the manifolds constructed in Lemma 10.4. Anyway, since our $(2 N-3)$-dimensional manifolds are sets with zero LEBESGUE measure in $\mathbb{R}^{2 N-2}$, the exceptional zero sets are contained in a set with zero LEBEsGue measure too. In the following, we denote the set of all exceptional zero sets by $E$.

It remains to show that the observation above can be transferred to the components of the corresponding complex-valued discrete-time signals with normalized support. For this purpose, we recall that the coefficients of a signal are determined (up to a multiplicative constant) by VIETA's formulae in Theorem 8.7 as discussed in Section 8. More precisely, if we take a multiplicative complex constant $C$ into account, then the components of the signal $x$ with normalized support of length $N$ can be written as

$$
x[N-1-n]=(-1)^{n} C S_{n}\left(\beta_{1}, \ldots, \beta_{N-1}\right),
$$

where $S_{n}$ denote the elementary symmetric functions in Definition 8.6. Hence, the relation between the corresponding zeros and the components of the signal is given by a continuously complex differentiable mapping from $\mathbb{C}^{N}$ to $\mathbb{C}^{N}$. 
In order to use SARD's theorem, we identify this relation with the real mapping $F: \mathbb{R}^{2 N} \rightarrow \mathbb{R}^{2 N}$ given by

$$
\begin{aligned}
& F\left(\mathfrak{R} \beta_{1}, \mathfrak{J} \beta_{1}, \ldots, \mathfrak{R} \beta_{N-1}, \mathfrak{J} \beta_{N-1}, \mathfrak{R} C, \mathfrak{J} C\right) \\
& \quad=(\mathfrak{R} x[0], \mathfrak{J} x[0], \ldots, \mathfrak{R} x[N-1], \mathfrak{J} x[N-1])^{\mathrm{T}} .
\end{aligned}
$$

The mapping $F$ is continuously differentiable since the relation between zeros and components of the signal $x$ is even continuously complex differentiable. If we extend the set $E$ of the exceptional corresponding zero sets by two further real components to describe the complex prefactor $C$, we can estimate the LEBESGUE measure of $F\left(E \times \mathbb{R}^{2}\right)$ with SARD's theorem (Theorem 10.5) by an integral over the extended set $E \times \mathbb{R}^{2}$. Since the CARTEsian product $E \times \mathbb{R}^{2}$ is a set with LEBESGUE measure zero, the image $F\left(E \times \mathbb{R}^{2}\right)$ has a zero LEBESGUE measure too.

All in all, we can thus conclude that the complex-valued signals that cannot be recovered from their FoURIER intensities and the modulus of its end point up to rotations are contained in a set of zero LEBESGUE measure in the real space $\mathbb{R}^{2 N}$. Because the remaining signals can be uniquely reconstructed up to rotations, the assertion follows.

\subsection{The modulus of an arbitrary signal value}

Based on the observations about the given modulus of the end point, we now investigate whether the given modulus of another signal value can also ensure the uniqueness of the discrete-time phase retrieval problem. Therefore, we generalize the findings from Section 10.1.

Firstly, we introduce the modified zero set

$$
\left\{\beta_{1}^{(\Lambda)}, \ldots, \beta_{N-1}^{(\Lambda)}\right\}
$$

for a subset $\Lambda$ of the original corresponding zero set $\left\{\beta_{1}, \ldots, \beta_{N-1}\right\}$. Here we define the single elements of the modified zero set by

$$
\beta_{j}^{(\Lambda)}:= \begin{cases}\bar{\beta}_{j}^{-1} & \beta_{j} \in \Lambda, \\ \beta_{j} & \text { else. }\end{cases}
$$

In other words, we obtain the modified zero set from the corresponding zero set by reflecting the zeros in the subset $\Lambda$ at the unit circle. 
Theorem 10.7. Let $x$ be a complex-valued discrete-time signal with normalized support of length $N$ and corresponding zero set $\left\{\beta_{1}, \ldots, \beta_{N-1}\right\}$. Then the signal $x$ can be uniquely recovered from its FOURIER intensity $|\widehat{x}|$ and the modulus of an arbitrary point $|x[N-1-\ell]|$ with $\ell$ between 0 and $N-1$ up to rotations if and only if the corresponding zeros $\beta_{j}$ and the modified zeros $\beta_{j}^{(\Lambda)}$ fulfil

$$
\left|S_{\ell}\left(\beta_{1}, \ldots, \beta_{N-1}\right)\right| \neq\left(\prod_{\beta_{j} \in \Lambda}\left|\beta_{j}\right|\right) \cdot\left|S_{\ell}\left(\beta_{1}^{(\Lambda)}, \ldots, \beta_{N-1}^{(\Lambda)}\right)\right|
$$

for each non-empty subset $\Lambda$ of the corresponding zero set $\left\{\beta_{1}, \ldots, \beta_{N-1}\right\}$ where $\Lambda$ does not contain reflected zero pairs or zeros on the unit circle.

Proof. The proof of Theorem 10.7 can be done in a similar way as the proof of Theorem 10.1. We start with an adapted version of the characterization of all discrete-time signals with the same FOURIER intensity in Theorem 5.1. Using the normalized support, we can thus write the original signal $x$ as

$$
x=\mathrm{e}^{\mathrm{i} \alpha} \sqrt{|a[N-1]| \prod_{j=1}^{N-1}\left|\beta_{j}\right|^{-1}} \cdot\left[{ }_{j=1}^{\mathbb{*}}\left(\delta_{1}-\beta_{j} \delta_{0}\right)\right],
$$

where $\alpha$ is an appropriate real number and $a$ is the autocorrelation signal of $x$. If we again identify the FOURIER transform of $x$ with an algebraic polynomial in the manner of Section 8 , then the absolute value of the considered point $x[N-1-\ell]$ is given by

$$
|x[N-1-\ell]|=\sqrt{|a[N-1]| \prod_{j=1}^{N-1}\left|\beta_{j}\right|^{-1}} \cdot\left|S_{\ell}\left(\beta_{1}, \ldots, \beta_{N-1}\right)\right|
$$

due to VIETA's formulae in Theorem 8.7.

Like in the proof of Theorem 10.1, we now assume that the signal $x$ cannot be uniquely recovered from its FoURIER intensity and the modulus of a certain point up to rotations. Hence, we find a further different solution $\breve{x}$ of the considered phase retrieval problem that is no rotation of the original signal. Moreover, we assume that the corresponding zeros of the second solution $\breve{x}$ can be obtained from the zero set $\left\{\beta_{1}, \ldots, \beta_{N-1}\right\}$ of the original signal $x$ by reflecting the zeros in the subset $\Lambda$ where $\Lambda$ does not contain reflected zero pairs or zeros on the unit circle. In other words, the signal $\breve{x}$ corresponds to the modified zero set $\left\{\beta_{1}^{(\Lambda)}, \ldots, \beta_{N-1}^{(\Lambda)}\right\}$. 
Since the absolute value $|\breve{x}[N-1-\ell]|$ can be represented analogously to $|x[N-1-\ell]|$ by substituting the corresponding zeros with the modified zeros, a comparison of both moduli yields the condition

$$
\begin{aligned}
& \sqrt{|a[N-1]| \prod_{j=1}^{N-1}\left|\beta_{j}\right|^{-1}} \cdot\left|S_{\ell}\left(\beta_{1}, \ldots, \beta_{N-1}\right)\right| \\
& \quad=\sqrt{|a[N-1]| \prod_{j=1}^{N-1}\left|\beta_{j}^{(\Lambda)}\right|^{-1}} \cdot\left|S_{\ell}\left(\beta_{1}^{(\Lambda)}, \ldots, \beta_{N-1}^{(\Lambda)}\right)\right| .
\end{aligned}
$$

Simplifying this equation by canceling the prefactor and bringing all zeros to the right-hand side, we finally have

$$
\left|S_{\ell}\left(\beta_{1}, \ldots, \beta_{N-1}\right)\right|=\left(\prod_{\beta_{j} \in \Lambda}\left|\beta_{j}\right|\right) \cdot\left|S_{\ell}\left(\beta_{1}^{(\Lambda)}, \ldots, \beta_{N-1}^{(\Lambda)}\right)\right| .
$$

Hence, the phase retrieval problem to recover the signal $x$ is only uniquely solvable up to rotations if and only if there exist no subset $\Lambda$ and hence no non-trivial ambiguity fulfilling the above condition.

If we consider a discrete-time signal $x$ with normalized support of odd length $N$, then we cannot recover $x$ from its FourIER intensity $|\widehat{x}|$ and the modulus $|x[(N-1) / 2]|$ of the central component in general because the absolute value of the central component obviously does not change under the reflection and conjugation of the complete signal. Hence, for $\Lambda$ chosen as the complete corresponding zero set, the uniqueness condition in Theorem 10.7 cannot hold true except when the zero set is invariant under the reflection at the unit circle.

To overcome this problem, we assume that the second solution $\breve{x}$ in the proof of Theorem 10.7 is additionally a non-trivially different ambiguity. Since the reflected and conjugated signal corresponds to the reflected zero set at the unit circle, see Lemma 5.3, we consequently consider only proper subsets $\Lambda$ of the corresponding zero set. More detailed, since the extension of $\Lambda$ by reflected zero pairs and zeros on the unit circle do not lead to a different non-trivial ambiguity, the subset $\Lambda$ should not be extendable to the complete set by adding zeros of this kind. Adapting the proof of Theorem 10.7 in this way, we obtain the following slightly weaker statement. 
Corollary 10.8. Let $x$ be a complex-valued discrete-time signal with normalized support of odd length $N$ and corresponding zero set $\left\{\beta_{1}, \ldots, \beta_{N-1}\right\}$. Then the signal $x$ can be uniquely recovered from its FOURIER intensity $|\widehat{x}|$ and the modulus of the point $|x[(N-1) / 2]|$ up to rotations and conjugate reflections if and only if the corresponding zeros $\beta_{j}$ and the modified zeros $\beta_{j}^{(\Lambda)}$ fulfil

$$
\left|S_{\frac{N-1}{2}}\left(\beta_{1}, \ldots, \beta_{N-1}\right)\right| \neq\left(\prod_{\beta_{j} \in \Lambda}\left|\beta_{j}\right|\right) \cdot\left|S_{\frac{N-1}{2}}\left(\beta_{1}^{(\Lambda)}, \ldots, \beta_{N-1}^{(\Lambda)}\right)\right|
$$

for each non-empty proper subset $\Lambda$ of the corresponding zero set $\left\{\beta_{1}, \ldots, \beta_{N-1}\right\}$ where $\Lambda$ does not contain reflected zero pairs or zeros on the unit circle and cannot be extended to the whole set by adding zeros of this kind.

In order to characterize the exceptional zero sets that do not satisfy the uniqueness conditions in Theorem 10.7 and Corollary 10.8, we again identify the corresponding zero set $\left\{\beta_{1}, \ldots, \beta_{N-1}\right\}$ with a real $(2 N-2)$-dimensional vector. Unfortunately, the direct construction of an appropriate manifold is much more challenging due to the additional elementary symmetric functions in the uniqueness conditions. Nevertheless, we can show that the exceptional zero sets lie on algebraic varieties or, more precisely, in the zero locus (the zero set) of a non-constant polynomial in $2 N-2$ indeterminates.

Lemma 10.9. If the corresponding zero set $\left\{\beta_{1}, \ldots, \beta_{N-1}\right\}$ of a complex-valued discrete-time signal is considered as real $(2 N-2)$-dimensional vector, then the zero sets satisfying

$$
\left|S_{\ell}\left(\beta_{1}, \ldots, \beta_{N-1}\right)\right|=\left(\prod_{\beta_{j} \in \Lambda}\left|\beta_{j}\right|\right) \cdot\left|S_{\ell}\left(\beta_{1}^{(\Lambda)}, \ldots, \beta_{N-1}^{(\Lambda)}\right)\right|
$$

lie in the zero locus of a non-constant polynomial for every non-empty subset $\Lambda$ of zeros $\beta_{j}$ or, when $\ell$ is equal to $(N-1) / 2$, for every proper subset $\Lambda$.

Proof. Analogously to the proof of Lemma 10.4, we identify the corresponding zero set $\left\{\beta_{1}, \ldots, \beta_{N-1}\right\}$ with the real $(2 N-2)$-dimensional vector

$$
\boldsymbol{\beta}:=\left(\mathfrak{R} \beta_{1}, \mathfrak{J} \beta_{1}, \ldots, \mathfrak{R} \beta_{N-1}, \mathfrak{J} \beta_{N-1}\right)^{\mathrm{T}} \in\left(\mathbb{R}^{2} \backslash\{0\}\right)^{N-1} .
$$

We investigate the set of all vectors $\boldsymbol{\beta}$ satisfying the equality condition (10.2) for a certain subset $\Lambda$ and a given integer $\ell$ between 0 and $N-1$. For this purpose, we 
square the equality condition and insert the definition of the elementary symmetric function (Definition 8.6), which leads us to

$$
\left|\sum_{1 \leq k_{1}<\cdots<k_{\ell} \leq N-1} \beta_{k_{1}} \cdots \beta_{k_{\ell}}\right|^{2}=\left|\prod_{\beta_{j} \in \Lambda} \bar{\beta}_{j}\right|^{2} \cdot\left|\sum_{1 \leq k_{1}<\cdots<k_{\ell} \leq N-1} \beta_{k_{1}}^{(\Lambda)} \cdots \beta_{k_{\ell}}^{(\Lambda)}\right|^{2},
$$

where we additionally conjugate the zeros in the prefactor on the right-hand side. As in the definition of the elementary symmetric functions, we set the empty sums for $\ell=0$ to one.

Next, we consider the substitution $\beta_{j}=\mathfrak{R} \beta_{j}+\mathrm{i} \mathfrak{J} \beta_{j}$. In this manner, the lefthand side of (10.3) becomes a real algebraic polynomial in $\boldsymbol{\beta}$ since the real and imaginary parts of the elementary symmetric function $S_{\ell}\left(\beta_{1}, \ldots, \beta_{N-1}\right)$ are real polynomials in $\mathfrak{R} \beta_{j}$ and $\mathfrak{J} \beta_{j}$. On the right-hand side, we also have a real algebraic polynomial in $\boldsymbol{\beta}$. More detailed, since the reflection of a zero $\beta_{j}$ at the unit circle is simply given by $\bar{\beta}_{j}^{-1}$, the cumbersome reflected zeros in the modified zero set completely cancels with the zeros in the prefactor. The real and imaginary parts of the products over the remaining modified and conjugated zeros are again real polynomials in $\mathfrak{R} \beta_{j}$ and $\mathfrak{J} \beta_{j}$. Hence, the right-hand side of (10.3) is really an algebraic polynomial in $\boldsymbol{\beta}$.

Since the polynomial obtained from the right-hand side of (10.3) also is well defined by continuous continuation whenever the real part $\mathfrak{R} \beta_{j}$ and imaginary part $\mathfrak{J} \beta_{j}$ of a corresponding zero $\beta_{j}$ are zero, we can neglect the assumption that the zeros $\beta_{j}$ of a discrete-time signal cannot be zero at the moment.

Bringing both polynomials to one side, we can already conclude that the vectors $\boldsymbol{\beta}$ satisfying (10.3) are the zero locus of an algebraic polynomial and hence an algebraic variety. In order to ensure that this polynomial is not constantly zero, we have to show that both sides of the polynomial equation (10.3) are nonequal. In order to simplify the following considerations, we assume without loss of generality that $\Lambda$ contains the first $J$ zeros of the corresponding zero set $\left\{\beta_{1}, \ldots, \beta_{N-1}\right\}$.

If we now determine the real or imaginary part of a summand

$$
\beta_{k_{1}} \cdots \beta_{k_{\ell}}=\left(\mathfrak{R} \beta_{k_{1}}+\mathrm{i} \mathfrak{J} \beta_{k_{1}}\right) \cdots\left(\mathfrak{R} \beta_{k_{\ell}}+\mathrm{i} \mathfrak{J} \beta_{k_{\ell}}\right)
$$

at the left-hand side of (10.3), we obtain a homogeneous polynomial of degree $\ell$ in the real variables $\mathfrak{R} \beta_{k_{1}}, \mathfrak{J} \beta_{k_{1}}, \ldots, \mathfrak{R} \beta_{k_{\ell}}, \mathfrak{J} \beta_{k_{\ell}}$. Hence, every monomial within the real polynomial obtained from the left-hand side of (10.3) has degree $2 \ell$. However, the degree of the monomials obtained from the right side of (10.3) varies depending on the numbers $\ell$ and $J$. More precisely, we distinguish the following three cases. 
(i) For numbers $\ell$ and $J$ with $\ell+J \leq N-1$, we always find increasing indicies $k_{1}<\cdots<k_{\ell}$ such that $k_{1}>J$. Then the product $\beta_{k_{1}}^{(\Lambda)} \cdots \beta_{k_{\ell}}^{(\Lambda)}$ on the righthand side of (10.3) simply becomes $\beta_{k_{1}} \cdots \beta_{k_{\ell}}$, and hence no zeros cancel with the prefactor. This implies that the corresponding monomials in the real version of (10.3) are exactly of degree $2(\ell+J)$. Since the monomials on the left-hand side are only of degree $2 \ell$, the polynomials on both sides of (10.3) cannot be equal.

(ii) If the numbers $\ell$ and $J$ fulfil $\ell+J>N-1$ and $J \leq \ell$, then we choose the indicies by

$$
k_{1}=1, \ldots, k_{\ell}=\ell \text {. }
$$

Now, the first $J$ modified zeros $\beta_{j}^{(\Lambda)}$ cancel with the prefactor, and the product $\beta_{J+1} \cdots \beta_{\ell}$ remains in the right-hand sum of (10.3). Since the real and imaginary parts of this summand consist of monomials of degree $\ell-J$, we have at least one monomial of degree $2(\ell-J)$ on the right-hand side of (10.3), which implies that the polynomials on the left-hand and right-hand side of (10.3) cannot cancel out.

(iii) In the following, we investigate the last case, where $\ell$ and $J$ satisfy $\ell+J>$ $N-1$ and $J>\ell$. Obviously, the polynomials on both sides of (10.3) can only cancel out if all monomials on the right-hand side are also of degree $2 \ell$. To show that this is impossible except for one special case, we consider the summands with indicies

$$
k_{1}=1, \ldots, k_{\ell}=\ell
$$

and

$$
k_{1}=N-\ell, \ldots, k_{\ell}=N-1 \text {. }
$$

Now, the first index set (10.4) yields the summand

$$
\bar{\beta}_{\ell+1} \cdots \bar{\beta}_{J}
$$

on the right-hand side since all modified zeros cancel with the prefactor. Thus, the corresponding squared real and imaginary parts occurring within the polynomial on the right-hand side of (10.3) can only be monomials of degree $2 \ell$ if the numbers $\ell$ and $J$ fulfil $J-\ell=\ell$ or $J=2 \ell$.

After possible cancelations with the prefactor, the second index set (10.5) corresponds to the summand

$$
\bar{\beta}_{1} \cdots \bar{\beta}_{N-\ell-1} \beta_{J+1} \cdots \beta_{N-1}
$$


with $2 N-2-\ell-J$ different variables. Here the assumption that the monomials of the squared real and imaginary part appearing on the righthand side of (10.3) have degree $2 \ell$ implies that $\ell$ and $J$ fulfil the equality $2 N-2-\ell-J=\ell$.

Combining the found restrictions on $\ell$ and $J$ from both sets of indicies directly yields

$$
\ell=\frac{N-1}{2} \text { and } \quad J=N-1 .
$$

Thus, the subset $\Lambda$ contains the complete corresponding zero set, which is not covered by the assertion. For all other cases, we can hence ensure that the polynomials on both sides of (10.3) cannot cancel each other.

All in all, the real algebraic polynomial in $\beta$ deduced from (10.3) by bringing both polynomials to one side is not constantly zero for all cases considered in the assertion, and the vectors $\boldsymbol{\beta}$ fulfilling condition (10.2) lie in the zero locus of this algebraic polynomial, which completes the proof.

Remark 10.10. The exceptional case in the proof of Lemma 10.9, where $\ell$ is equal to $(N-1) / 2$, and where $\Lambda$ contains the complete zero set, corresponds to the assumption that the moduli $|x[(N-1) / 2]|$ and $|\breve{x}[(N-1) / 2]|$ of the original and the conjugated, reflected signal are equal, as mentioned in Corollary 10.8. Since this is always the case, the polynomial equation (10.3) has to be fulfilled for every vector $\boldsymbol{\beta}$.

Formally, this observation directly follows from the identity

$$
\bar{\beta}_{1} \cdots \bar{\beta}_{N-1} \cdot S_{\frac{N-1}{2}}\left(\bar{\beta}_{1}^{-1}, \ldots, \bar{\beta}_{N-1}^{-1}\right)=S_{\frac{N-1}{2}}\left(\bar{\beta}_{1}, \ldots, \bar{\beta}_{N-1}\right),
$$

which can be shown in a similar way to Lemma 9.3. More precisely, the uniqueness condition in (10.3) is equivalent to

$$
\left|S_{\frac{N-1}{2}}\left(\beta_{1}, \ldots, \beta_{N-1}\right)\right|^{2}=\left|S_{\frac{N-1}{2}}\left(\bar{\beta}_{1}, \ldots, \bar{\beta}_{N-1}\right)\right|^{2} .
$$

Since the additional conjugations on the right-hand side vanish under the absolute value, the constructed real algebraic polynomial in the proof of Lemma 10.9 is indeed constantly zero.

The main difference between Lemma 10.4 and Lemma 10.9 is that we have no explicit characterization of the exceptional zero sets whose corresponding signals cannot be uniquely recovered from their FoURIER intensities and the modulus of an arbitrary point. Nevertheless, we can use the constructed algebraic vari- 
eties in Lemma 10.9 to show that almost every signal can be recovered uniquely up to rotations and possibly conjugate reflections.

Theorem 10.11. Let $x$ be a complex-valued discrete-time signal with normalized support of length $N$, and let $\ell$ be an arbitrary integer between 0 and $N-1$. The phase retrieval problem to recover the signal $x$ from its FOURIER intensity $|\widehat{x}|$ and the absolute value $|x[N-1-\ell]|$ is almost always uniquely solvable up to rotations whenever $\ell \neq(N-1) / 2$. In the special case that $\ell=(N-1) / 2$, the reconstruction is only unique up to rotations and conjugate reflections.

Proof. As a generalization of Theorem 10.6, we can show the assertion by adapting the corresponding proof. Interpreting the corresponding zero set as a real $(2 N-2)$-dimensional vector, we have shown in Lemma 10.9 that the zero sets violating the uniqueness conditions in Theorem 10.7 and Corollary 10.8 for a certain subset $\Lambda$ and number $\ell$ are contained in the zero locus of a non-constant algebraic polynomial.

Consequently, the exceptional zero sets of one uniqueness condition form a null set as part of a zero locus with zero LEBESGuE measure. Since we have only finitely many uniqueness conditions, the union of all exceptional zero sets is only a set with zero LEBESGUE measure too.

With the aid of VIETA's formulae (Theorem 8.7) and SARD's theorem (Theorem 10.5), we can transfer this observation to the components of the corresponding discrete-time signals as shown in the proof of Theorem 10.6. Hence, almost every signal with normalized support of length $N$ fulfils the uniqueness conditions in Theorem 10.7 and Corollary 10.8.

\subsection{The moduli of the entire signal}

Looking back at our findings in Section 10.1 and Section 10.2, where we prove that almost every signal can be uniquely recovered up to rotations with the aid of an additional modulus in the time domain, we investigate the question: can the phase retrieval problem to recover the signal $x$ be uniquely solved up to rotations if more then one modulus $|x[n]|$ or even all moduli $(|x[n]|)_{n \in \mathbb{Z}}$ are given?

Phase retrieval problems of this kind, where the complete modulus of the signal is known, have been studied in [GS72] and [SSD ${ }^{+}$o6, LTo8, LTo9], for instance. In the first one [GS72], GERCHBERG and SAXTON present an alternating projection method to solve this problem numerically. Afterwards, SEIFERT, Stolz, Donatelli, LANGemann, and Tasche in [SSD ${ }^{+}$o6] as well as LANGemann and TASche in [LTo8, LTo9] propose a multilevel GAUSs-NewTon method as a 
numerical approach. In the considered examples, both algorithms can recover the unknown signal with high accuracy from its FouRIER intensity and its moduli up to rotations.

Based on the uniqueness results for the phase retrieval problem with one additionally given modulus of an arbitrary point in Theorem 10.11, we can immediately justify this behaviour of the algorithms also theoretically. More precisely, non-trivial ambiguities arise in this setting rarely as stated in the following corollary.

Corollary 10.12. Let $x$ be a complex-valued discrete-time signal $x$ with normalized support of length $N$. The phase retrieval problem to recover the signal $x$ from its FOURIER intensity $|\widehat{x}|$ and its moduli $(|x[n]|)_{n \in \mathbb{Z}}$ is almost always uniquely solvable up to rotations.

Unfortunately, the additional knowledge of more than one modulus of the signal in time domain cannot ensure uniqueness of the corresponding phase retrieval problem generally, even if the complete modulus $|x|:=(x[n])_{n \in \mathbb{Z}}$ of the signal $x$ is given. In other words, the exceptional set of signals that cannot be uniquely recovered up to rotations in Corollary 10.12 is always non-empty. Before we construct specific counter-examples for every possible signal length, we analyze the following example.

Example 10.13. We consider the discrete-time phase retrieval problem to recover the signal

$$
x:=\frac{1}{2}(\ldots, 0, \underline{-2+2 \mathrm{i}}, 2 \mathrm{i},-1-\mathrm{i}, 2,0, \ldots)
$$

from its FourIER intensity $|\widehat{x}|$ and its modulus $|x|$. As seen in Theorem 10.1, the phase retrieval problem to recover $x$ can only possesses non-trivial ambiguities if the corresponding zero set contains a subset whose product is unimodular.

Since the corresponding zeros of the signal $x$ are given by

$$
\beta_{1}=-\frac{1}{2}-\frac{1}{2} \mathrm{i}, \quad \beta_{2}=-\mathrm{e}^{-\mathrm{i} \frac{2 \pi}{3}}(1+\mathrm{i}), \quad \text { and } \quad \beta_{3}=-\mathrm{e}^{\mathrm{i} \frac{2 \pi}{3}}(1+\mathrm{i}),
$$

the considered phase retrieval problem can have up to two further non-trivial ambiguities as the two products $\beta_{1} \beta_{2}$ and $\beta_{1} \beta_{3}$ have an absolute value equal to one. In this specific example, both non-trivial ambiguities

$$
y_{1}=\left(\ldots, 0, x[0], \mathrm{e}^{\mathrm{i} \frac{2 \pi}{3}} x[1], \mathrm{e}^{-\mathrm{i} \frac{2 \pi}{3}} x[2], x[3], 0, \ldots\right)
$$




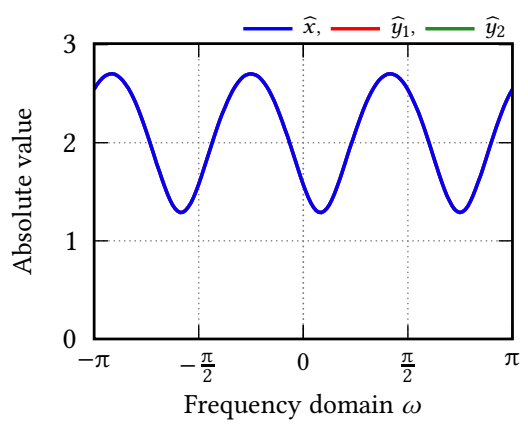

(a) Fourier intensities of the signals $x$, $y_{1}$, and $y_{2}$

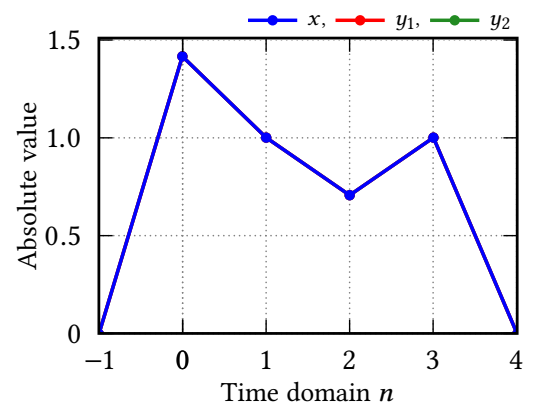

(c) Absolute value of the signals $x$, $y_{1}$ and $y_{2}$ illustrated by polygonal lines

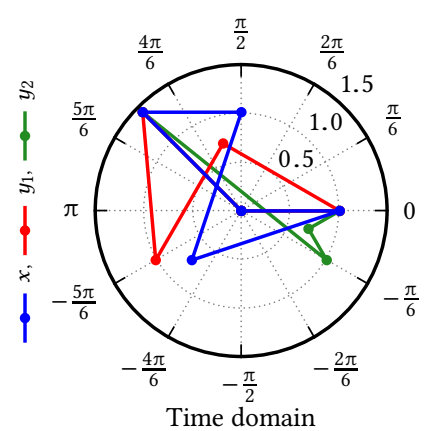

(b) Signals $x, y_{1}$, and $y_{2}$ represented in polar form

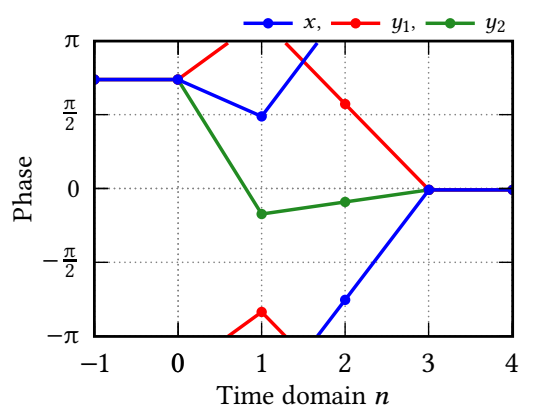

(d) Phase of the signals $x, y_{1}$, and $y_{2}$ illustrated by polygonal lines

Figure 10.1.: Discrete-time phase retrieval problem to recover the signal $x$ from its Fourier intensity and its moduli with two further non-trivial ambiguities

and

$$
y_{2}=\left(\ldots, 0, x[0], \mathrm{e}^{-\mathrm{i} \frac{2 \pi}{3}} x[1], \mathrm{e}^{\mathrm{i} \frac{2 \pi}{3}} x[2], x[3], 0, \ldots\right),
$$

which are constructed by reflecting the subsets $\left\{\beta_{1}, \beta_{2}\right\}$ or $\left\{\beta_{1}, \beta_{3}\right\}$ respectively, obviously have the same modulus as the original signal $x$. The original signal and both occurring non-trivial ambiguities are shown in Figure 10.1.

The example above not only shows that the statement in Corollary 10.12 cannot be tightened, but also that the constructed algebraic varieties in Lemma 10.9 are really non-empty. This observation is also valid for all greater signal lengths. 
Theorem 10.14. For every integer $N>3$, there exists a discrete-time signal $x$ with normalized support of length $N$ such that the phase retrieval problem to recover this signal $x$ from its FOURIER intensity and its moduli possesses at least a further nontrivial ambiguity.

Proof. In order to construct a discrete-time signal $x$ that cannot be uniquely recovered from its FourIER intensity and its moduli, we investigate the uniqueness conditions in Theorem 10.7 for a specifically chosen zero set $\left\{\beta_{1}, \ldots, \beta_{N-1}\right\}$. More precisely, we assume that $\beta_{1}$ and $\beta_{2}$ differ, and that the remaining corresponding zeros $\beta_{3}, \ldots, \beta_{N-1}$ coincide. In other words, we assume that $\beta_{3}$ is a zero of multiplicity $N-3$.

In the following, we try to choose the three zeros in a way such that the ambiguity $y$ obtained by reflecting the zeros $\beta_{1}$ and $\beta_{2}$ is a further non-trivial solution. Since Theorem 10.1 implies that the product of $\beta_{1}$ and $\beta_{2}$ must be unimodular, we assume that $\beta_{2}$ is of the form

$$
\beta_{2}:=\mathrm{e}^{-\mathrm{i} \phi} \bar{\beta}_{1}^{-1}
$$

for an appropriate real number $\phi$. This definition yields the three identities

$$
\beta_{2}^{-1}=\bar{\beta}_{1} \bar{\beta}_{2} \beta_{1} \quad \text { and } \quad \beta_{1}^{-1}=\bar{\beta}_{1} \bar{\beta}_{2} \beta_{2}
$$

and hence

$$
\beta_{1}^{-1}+\beta_{2}^{-1}=\bar{\beta}_{1} \bar{\beta}_{2}\left(\beta_{1}+\beta_{2}\right)
$$

To ensure that both moduli $|x[N-1-\ell]|$ and $|y[N-1-\ell]|$ coincide for an integer $\ell$ between 0 and $N-1$, the corresponding zeros have to fulfil the equality

$$
\left|S_{\ell}\left(\beta_{1}, \ldots, \beta_{N-1}\right)\right|^{2}=\left|\bar{\beta}_{1} \bar{\beta}_{2}\right|^{2} \cdot\left|S_{\ell}\left(\bar{\beta}_{1}^{-1}, \bar{\beta}_{2}^{-1}, \beta_{3}, \ldots, \beta_{N-1}\right)\right|^{2},
$$

where $S_{\ell}$ denotes the $\ell$ th elementary symmetric function given in Definition 8.6, as seen in Theorem 10.7 and Corollary 10.8. Consequently, if the above condition holds for every possible $\ell$, then all moduli of $|x|$ and $|y|$ are equal.

In the next step, we simplify this condition by factoring out $\beta_{1}, \beta_{2}$ and their reflections from the elementary symmetric functions $S_{\ell}$ on both sides. With the notation $\Pi_{\ell}:=S_{\ell}\left(\beta_{3}, \ldots, \beta_{N-1}\right)$ for the elementary symmetric functions of the remaining zeros, the equation becomes

$$
\left|\beta_{1} \beta_{2} \Pi_{\ell-2}+\left(\beta_{1}+\beta_{2}\right) \Pi_{\ell-1}+\Pi_{\ell}\right|^{2}=\left|\Pi_{\ell-2}+\left(\bar{\beta}_{1}+\bar{\beta}_{2}\right) \Pi_{\ell-1}+\bar{\beta}_{1} \bar{\beta}_{2} \Pi_{\ell}\right|^{2} .
$$


Further, we extend the squared absolute values with the law of cosines. Since the corresponding summands on both sides obviously have the same absolute value, only the mixed terms remain. Using the identities (10.7) and (10.8), and canceling the equal terms on both sides, we have

$$
\begin{aligned}
& \mathfrak{R}\left[\left(\bar{\beta}_{1}^{-1}+\bar{\beta}_{2}^{-1}\right) \Pi_{\ell-2} \bar{\Pi}_{\ell-1}+\left(\beta_{1}+\beta_{2}\right) \Pi_{\ell-1} \bar{\Pi}_{\ell}\right] \\
& \quad=\mathfrak{R}\left[\left(\beta_{1}+\beta_{2}\right) \Pi_{\ell-2} \bar{\Pi}_{\ell-1}+\left(\bar{\beta}_{1}^{-1}+\bar{\beta}_{2}^{-1}\right) \Pi_{\ell-1} \bar{\Pi}_{\ell}\right] .
\end{aligned}
$$

Remembering that the zeros $\beta_{3}, \ldots, \beta_{N-1}$ coincide, we can explicitly compute the appearing elementary symmetric functions. In particular, we have

$$
\Pi_{\ell}=\left(\begin{array}{c}
N-3 \\
\ell
\end{array}\right) \beta_{3}^{\ell} \quad \text { and hence } \quad \Pi_{\ell-1} \bar{\Pi}_{\ell}=\left(\begin{array}{c}
N-3 \\
\ell-1
\end{array}\right)\left(\begin{array}{c}
N-3 \\
\ell
\end{array}\right)\left|\beta_{3}\right|^{2 \ell-2} \bar{\beta}_{3} .
$$

If the real parts on the left and right-hand side of (10.9) do not cancel each other completely, which does not happen when $\ell$ is equal to one, then the nonuniqueness condition (10.9) is equivalent to

$$
\mathfrak{R}\left[\left(\beta_{1}+\beta_{2}\right) \bar{\beta}_{3}\right]=\mathfrak{R}\left[\left(\bar{\beta}_{1}^{-1}+\bar{\beta}_{2}^{-1}\right) \bar{\beta}_{3}\right] .
$$

On the contrary, if we choose the zeros $\beta_{1}, \beta_{2}$, and $\beta_{3}$ such that they satisfy this equation, then the non-uniqueness condition (10.9) holds true for every $\ell$ between 0 and $N-1$.

In order to find a suitable corresponding zero set, we replace $\beta_{2}$ in the last equation by its definition (10.6). This substitution yields the condition

$$
\mathfrak{R}\left[\left(\beta_{1}+\mathrm{e}^{-\mathrm{i} \phi} \bar{\beta}_{1}^{-1}\right) \bar{\beta}_{3}\right]=\mathfrak{R}\left[\left(\bar{\beta}_{1}^{-1}+\mathrm{e}^{-\mathrm{i} \phi} \beta_{1}\right) \bar{\beta}_{3}\right] .
$$

Due to the fact that the reflected zero can be written as $\bar{\beta}_{1}^{-1}=\left|\beta_{1}\right|^{-2} \beta_{1}$, a rearrangement of the summands leads to

$$
\left(1-\frac{1}{\left|\beta_{1}\right|^{2}}\right) \mathfrak{R}\left[\beta_{1} \bar{\beta}_{3}\right]=\left(1-\frac{1}{\left|\beta_{1}\right|^{2}}\right) \mathfrak{R}\left[\mathrm{e}^{-\mathrm{i} \phi} \beta_{1} \bar{\beta}_{3}\right]
$$

Writing the product $\beta_{1} \bar{\beta}_{3}$ in its polar form $\left|\beta_{1} \bar{\beta}_{3}\right| \mathrm{e}^{\mathrm{i} \psi}$ for a suitable real number $\psi$, and canceling the equal prefactor on both sides, we finally obtain

$$
\mathfrak{R} \mathrm{e}^{\mathrm{i} \psi}=\mathfrak{R} \mathrm{e}^{\mathrm{i}(\psi-\phi)} \quad \text { or } \quad \cos (\psi)=\cos (\psi-\phi) .
$$


Since the cosine is an even, $2 \pi$-periodic function, this trigonometric equation has up to two different solutions except for additive multiples of $2 \pi$. The first one is obviously $\phi=0$, but then $\beta_{1}$ and $\beta_{2}$ form a reflected pair with respect to the unit circle, and hence the original signal $x$ and the ambiguity $y$ coincide. The only other arising solution is given by $\phi=2 \psi$, which implies that $\beta_{2}$ completely depends on $\beta_{1}$ and $\beta_{3}$ by

$$
\beta_{2}=\mathrm{e}^{-2 \mathrm{i} \psi} \bar{\beta}_{1}^{-1} \quad \text { or } \quad \beta_{2}=\mathrm{e}^{-2 \mathrm{i} \arg \left(\beta_{1} \bar{\beta}_{3}\right)} \bar{\beta}_{1}^{-1} .
$$

If we now choose the zeros $\beta_{1}$ and $\beta_{3}$ not lying on the unit circle so that the imaginary part of $\beta_{1} \bar{\beta}_{3}$ does not vanish, we can avoid that $\beta_{1}$ and $\beta_{2}$ form a reflected zero pair. Therefore, the ambiguity $y$ is non-trivially different from the original signal $x$ by construction, which leads to the assertion.

\section{Using additional phase information}

With the knowledge that the modulus of an arbitrary point in the time domain contains enough information to enforce the uniqueness of the phase retrieval problem for almost every signal up to trivial ambiguities, the question arises whether a priori phase information about the unknown signal can also enforce uniqueness. Obviously, the phase of only one component of the signal is completely useless because of the trivial rotation ambiguity, see Proposition 2.1. Hence, we need at least the phase of two components.

If we look back at Example 6.7, where we consider a phase retrieval problem with the maximal number of non-trivial ambiguities, we can observe that the phases of all ambiguities in both end points coincide, see Figure 6.3(d) in particular. To justify this observation formally, we consider the right and left end point of a discrete-time signal given by

$$
x[N-1]=\mathrm{e}^{\mathrm{i} \alpha} \sqrt{|a[N-1]| \prod_{j=1}^{N-1}\left|\beta_{j}\right|^{-1}}
$$

and

$$
x[0]=(-1)^{N-1} \mathrm{e}^{\mathrm{i} \alpha} \sqrt{|a[N-1]| \prod_{j=1}^{N-1}\left|\beta_{j}\right|^{-1}} \cdot \prod_{j=1}^{N-1} \beta_{j}
$$


as characterized in Theorem 5.1. The end points of the non-trivial ambiguities are given in an analogous form, where a subset of the corresponding zero set $\left\{\beta_{1}, \ldots, \beta_{N-1}\right\}$ is reflected at the unit circle. Since the additional rotation by $\alpha$ can individually be chosen for each non-trivial ambiguity, we can assume without loss of generality that the phase of the right end point of all non-trivial ambiguities coincide. Using that the phase of a complex number is invariant under reflection at the unit circle, we can further conclude that the phase of the left end point of all non-trivial ambiguities are also equal as conjectured.

Nonetheless, if we consider the phase of the right end point and of another inner point, then the previous examples imply that the phase of these points for different non-trivial ambiguities coincides only for rare exceptions. In the next subsection, we will show that already the additional phase information for two components of the unknown signal can reduce the set of non-trivial ambiguities in a suitable manner.

\subsection{Phase of an arbitrary point and the end point}

Before we investigate the uniqueness of the discrete-time phase retrieval problem with two arbitrary given phases in the time domain, we consider the slightly simpler version where one given phase arises from the right end point. In order to show that this additional phase information can indeed enforce a unique reconstruction for almost every signal, we proceed analogously to the approach in Section 10. This means that we firstly characterize the signals that cannot be uniquely reconstructed, show that the exceptional zero sets are contained in an appropriate algebraic variety of lower dimension, and finally conclude that non-trivial ambiguities can only arise in rare special cases.

In order to simplify the notation in the following, we denote the complete corresponding zero set and the modified zero set defined in Section 10.2 by

$$
B:=\left\{\beta_{1}, \ldots, \beta_{N-1}\right\} \quad \text { and } \quad B^{(\Lambda)}:=\left\{\beta_{1}^{(\Lambda)}, \ldots, \beta_{N-1}^{(\Lambda)}\right\}
$$

respectively. Further, since the elementary symmetric functions in Definition 8.6 are symmetric polynomials and hence invariant under permutations of their arguments, we define the elementary symmetric functions of a set by

$$
S_{n}(B):=S_{n}\left(\beta_{1}, \ldots, \beta_{N-1}\right) \quad \text { and } \quad S_{n}\left(B^{(\Lambda)}\right):=S_{n}\left(\beta_{1}^{(\Lambda)}, \ldots, \beta_{N-1}^{(\Lambda)}\right)
$$

for every integer $n$. 
Theorem 11.1. Let $x$ be a complex-valued discrete-time signal with normalized support of length $N$ and corresponding zero set $B:=\left\{\beta_{1}, \ldots, \beta_{N-1}\right\}$, and let $\ell$ be an integer between 1 and $N-2$. Then the signal $x$ cannot be uniquely recovered from its FOURIER intensity $|\widehat{x}|$ and the two phases $\arg x[N-1]$ and $\arg x[N-1-\ell]$ if and only if there exists a non-empty subset $\Lambda$ of the corresponding zero set $B$, where $\Lambda$ does not contain reflected zero pairs or zeros on the unit circle, such that the corresponding zero set $B$ and the modified zero set $B^{(\Lambda)}$ fulfil

$$
\mathfrak{R} S_{\ell}(B) \mathfrak{I} S_{\ell}\left(B^{(\Lambda)}\right)-\mathfrak{J} S_{\ell}(B) \mathfrak{R} S_{\ell}\left(B^{(\Lambda)}\right)=0
$$

and further

$$
\mathfrak{R} S_{\ell}(B) \mathfrak{R} S_{\ell}\left(B^{(\Lambda)}\right)+\mathfrak{J} S_{\ell}(B) \mathfrak{J} S_{\ell}\left(B^{(\Lambda)}\right) \geq 0
$$

Proof. Firstly, we assume that the phase retrieval problem to recover $x$ from its FourIER intensity and the two phases $\arg x[N-1-\ell]$ and $\arg x[N-1]$ possesses at least one further non-trivially different solution $y$. Therefore, we find a subset $\Lambda$ of the corresponding zero set $B$ of the original signal $x$ such that the second solution $y$ corresponds to the modified zero set $B^{(\Lambda)}$. Since $x$ and $y$ differ nontrivially, we can assume that $\Lambda$ is non-empty and does not contain reflected zero pairs or zeros on the unit circle.

Once again, the end point of the signal $x$ is given by

$$
x[N-1]=\mathrm{e}^{\mathrm{i} \alpha} \sqrt{|a[N-1]| \prod_{j=1}^{N-1}\left|\beta_{j}\right|^{-1}},
$$

where $\alpha$ is a real number and $a[N-1]$ denotes the $(N-1)$ st coefficient of the autocorrelation signal of $x$, see Theorem 5.1. Since the end point of the ambiguity $y$ is analogously given with the modified zero set, and since the phase of the end point only depends on $\alpha$, we can conclude that $x$ and $y$ have to have the same rotation factor $\mathrm{e}^{\mathrm{i} \alpha}$.

Next, we examine the restrictions on the corresponding zero set given by the second phase $\arg x[N-1-\ell]$. For this purpose, we recall that the components of a discrete-time signal with normalized support are given by

$$
x[N-1-\ell]=(-1)^{\ell} \mathrm{e} i \sqrt{|a[N-1]| \prod_{j=1}^{N-1}\left|\beta_{j}\right|^{-1}} \cdot S_{\ell}(B)
$$

due to VIETA's formulae as discussed in Section 8. For the component $y[N-1-\ell]$ 
of the ambiguity, we have an analogous representation where the corresponding zero set is replaced by the modified. Since the rotation factors $\mathrm{e}^{\mathrm{i} \alpha}$ coincide as observed above, the corresponding phases of $x$ and $y$ only depend on the phase of the elementary symmetric function $S_{\ell}$. In other words, the phase condition

$$
\arg x[N-1-\ell]=\arg y[N-1-\ell]
$$

is equivalent to

$$
\arg S_{\ell}(B)=\arg S_{\ell}\left(B^{(\Lambda)}\right)
$$

Hence, the function values $S_{\ell}(B)$ and $S_{\ell}\left(B^{(\Lambda)}\right)$ have the same phase if and only if $S_{\ell}\left(B^{(\Lambda)}\right)$ lies on the real ray from the origin through $S_{\ell}(B)$ in the complex plane. Formally, $S_{\ell}\left(B^{(\Lambda)}\right)$ thus has to satisfy the equation of a straight line

$$
\mathfrak{R} S_{\ell}(B) \mathfrak{I} S_{\ell}\left(B^{(\Lambda)}\right)-\mathfrak{I} S_{\ell}(B) \mathfrak{R} S_{\ell}\left(B^{(\Lambda)}\right)=0 .
$$

Moreover, to ensure that $S_{\ell}(B)$ and $S_{\ell}\left(B^{(\Lambda)}\right)$ lie on the same half line, the angle between the $S_{\ell}(B)$ and $S_{\ell}\left(B^{(\Lambda)}\right)$ have to be less than $\pi / 2$, and hence the real scalar product of $S_{\ell}(B)$ and $S_{\ell}\left(B^{(\Lambda)}\right)$ must fulfil

$$
\mathfrak{R} S_{\ell}(B) \mathfrak{R} S_{\ell}\left(B^{(\Lambda)}\right)+\mathfrak{J} S_{\ell}(B) \mathfrak{I} S_{\ell}\left(B^{(\Lambda)}\right) \geq 0 .
$$

This slightly cumbersome additional condition guarantees that the assertion also holds when one or both signal values $x[N-1-\ell]$ or $y[N-1-\ell]$ are zero, and the corresponding phases are not uniquely defined.

Like the approach in Section 10.2, we can observe that the corresponding zero sets of signals that cannot be uniquely reconstructed are covered by the union of specific algebraic varieties. To show this, we again identify the corresponding zero set $\left\{\beta_{1}, \ldots, \beta_{N-1}\right\}$ of a discrete-time signal with the $(2 N-2)$-dimensional vector

$$
\left(\mathfrak{R} \beta_{1}, \mathfrak{J} \beta_{1}, \ldots, \mathfrak{R} \beta_{N-1}, \mathfrak{J} \beta_{N-1}\right)^{\mathrm{T}}
$$

For simplicity, we only investigate the equation of a straight line in Theorem 11.1 and neglect the inequality condition.

Lemma 11.2. If the corresponding zero set $B:=\left\{\beta_{1}, \ldots, \beta_{N-1}\right\}$ of a complexvalued discrete-time signal is considered as a real $(2 N-2)$-dimensional vector, then the zero sets satisfying

$$
\mathfrak{R} S_{\ell}(B) \mathfrak{J} S_{\ell}\left(B^{(\Lambda)}\right)-\mathfrak{I} S_{\ell}(B) \mathfrak{R} S_{\ell}\left(B^{(\Lambda)}\right)=0
$$


lie in the zero locus of a non-constant polynomial for every non-empty subset $\Lambda$ of $B$ and $\ell$ between 1 and $N-2$.

Proof. In order to construct the real algebraic variety, we replace the complex variables $\beta_{j}$ in the linear equation by their algebraic form $\mathfrak{R} \beta_{j}+\mathrm{i} \mathfrak{J} \beta_{j}$. In this manner, the real and imaginary parts of the elementary symmetric function $S_{\ell}(B)$ become real polynomials in the indeterminates $\mathfrak{R} \beta_{j}$ and $\mathfrak{J} \beta_{j}$.

Remembering that the real and imaginary parts of reflected zeros at the unit circle are given by

$$
\mathfrak{R} \bar{\beta}_{j}^{-1}=\frac{\mathfrak{R} \beta_{j}}{\left[\mathfrak{R} \beta_{j}\right]^{2}+\left[\mathfrak{I} \beta_{j}\right]^{2}} \quad \text { and } \quad \mathfrak{J} \bar{\beta}_{j}^{-1}=\frac{\mathfrak{I} \beta_{j}}{\left[\mathfrak{R} \beta_{j}\right]^{2}+\left[\mathfrak{I} \beta_{j}\right]^{2}}
$$

we see that the real and imaginary parts of the elementary symmetric function

$$
S_{\ell}\left(B^{(\Lambda)}\right)=\sum_{1 \leq k_{1}<\cdots<k_{n} \leq N-1}\left(\mathfrak{R} \beta_{k_{1}}^{(\Lambda)}+\mathrm{i} \mathfrak{J} \beta_{k_{1}}^{(\Lambda)}\right) \cdots\left(\mathfrak{R} \beta_{k_{\ell}}^{(\Lambda)}+\mathrm{i} \mathfrak{J} \beta_{k_{\ell}}^{(\Lambda)}\right)
$$

of the modified zero sets are rational polynomials, where the denominator of the individual summands contains the moduli of the reflected zeros. Hence, if we multiply the equation (11.1) with

$$
\Pi_{\Lambda}:=\prod_{\beta_{j} \in \Lambda}\left(\left[\mathfrak{R} \beta_{j}\right]^{2}+\left[\mathfrak{J} \beta_{j}\right]^{2}\right)
$$

all occurring inverse moduli cancel out, and we obtain the equivalent condition

$$
\Pi_{\Lambda}\left(\mathfrak{R} S_{\ell}(B) \mathfrak{J} S_{\ell}\left(B^{(\Lambda)}\right)-\mathfrak{J} S_{\ell}(B) \mathfrak{R} S_{\ell}\left(B^{(\Lambda)}\right)\right)=0
$$

with an algebraic polynomial in the indeterminates $\mathfrak{R} \beta_{j}$ and $\mathfrak{J} \beta_{j}$ on the left-hand side.

Analogously as in the proof of Lemma 10.9, the issue arises whether the algebraic polynomial in (11.2) is trivial or not. To see this, we extend the polynomial continuously whenever a zero division in the original linear equation occurs; so we can neglect that the corresponding zero sets of a discrete-time signal cannot contain zero elements. To simplify the following considerations, we assume without loss of generality that $\Lambda$ contains the first $J$ zeros of the corresponding zero set $B$.

Let us first consider the real and imaginary part of one summand $\beta_{k_{1}} \cdots \beta_{k_{\ell}}$ in the definition of the elementary symmetric function $S_{\ell}$. As discussed above, the real and imaginary part consists of monomials of degree $\ell$ in the indeterminates 
$\mathfrak{R} \beta_{j}$ and $\mathfrak{J} \beta_{j}$. Moreover, the monomials of the real part $\mathfrak{R}\left[\beta_{k_{1}} \cdots \beta_{k_{\ell}}\right]$ always have an even number of 'imaginary variables' $\mathfrak{J} \beta_{j}$, whereas the monomials of the imaginary part $\mathfrak{J}\left[\beta_{k_{1}} \cdots \beta_{k_{\ell}}\right]$ have an odd number. This observation inductively follows from the multiplication of two complex numbers in their algebraic form. Hence, the monomials in the real and imaginary part of $\beta_{k_{1}} \cdots \beta_{k_{\ell}}$ are pairwise different.

In order to show that the polynomial (11.2) cannot vanish everywhere, we use the following approach: we choose a specific monomial in the left summand

$$
\Pi_{\Lambda} \mathfrak{R} S_{\ell}(B) \mathfrak{J} S_{\ell}\left(B^{(\Lambda)}\right)
$$

and show that there does not exist a corresponding monomial in the right summand

$$
\Pi_{\Lambda} \mathfrak{J} S_{\ell}(B) \mathfrak{R} S_{\ell}\left(B^{(\Lambda)}\right)
$$

If we find such a monomial that only occurs in the left summand, then the polynomial in (11.2) possesses at least this one monomial and cannot be trivial. Here we distinguish the following two major cases.

(i) Firstly, we assume that $N-1>\ell \geq J \geq 1$ and consider the specific monomial

$$
\mathfrak{J} \beta_{1}\left[\mathfrak{R} \beta_{2}\right]^{2} \cdots\left[\mathfrak{R} \beta_{\ell}\right]^{2} \mathfrak{R} \beta_{\ell+1}
$$

In order to show that this monomial really appears in the left summand $\Pi_{\Lambda} \mathfrak{R} S_{\ell}(B) \mathfrak{J} S_{\ell}\left(B^{(\Lambda)}\right)$ of (11.2), we determine the corresponding factor for each variable in (11.3). Due to the fact that no mixed terms of the form $\mathfrak{R} \beta_{j} \mathfrak{J} \beta_{j}$ occur, it is enough to assign the indicies of the variables to the three factors.

Since the degree is obviously $2 \ell$, the considered monomial cannot contain any quadratic factor $\left[\mathfrak{R} \beta_{j}\right]^{2}$ or $\left[\mathfrak{J} \beta_{j}\right]^{2}$ from the prefactor $\Pi_{\Lambda}$. Consequently, the prefactor $\Pi_{\Lambda}$ and the inverse moduli in $\mathfrak{J S} S_{\ell}\left(B^{(\Lambda)}\right)$ have to cancel each other, and the index set

$$
\breve{K}:=\left\{\breve{k}_{1}, \ldots, \breve{k}_{\ell}\right\}
$$

of the monomial from $\mathfrak{J} S_{\ell}\left(B^{(\Lambda)}\right)$ must contain the indicies from 1 to $J$. Further, the index set

$$
K:=\left\{k_{1}, \ldots, k_{\ell}\right\}
$$

of the monomial from $\mathfrak{R} S_{\ell}(B)$ must also comprise the indicies from 2 to $J$ in order to cover the remaining variables $\mathfrak{R} \beta_{2}, \ldots, \mathfrak{R} \beta_{J}$ of the quadratic 
factors. With the same argument, both index sets have to contain the indicies from $J+1$ to $\ell$. For the reason that the index set $\breve{K}$ already comprises $\ell$ indicies, the last index of the variable $\mathfrak{R} \beta_{\ell+1}$ must be contained in $K$.

All in all, we can conclude that the monomial (11.3) uniquely arises from the factors

$$
\mathfrak{R} \beta_{2} \cdots \mathfrak{R} \beta_{\ell+1}
$$

in $\mathfrak{R} S_{\ell}(B)$ and

$$
\frac{\mathfrak{J} \beta_{1} \mathfrak{R} \beta_{2} \cdots \mathfrak{R} \beta_{\ell}}{\prod_{j=1}^{J}\left(\left[\mathfrak{R} \beta_{j}\right]^{2}+\left[\mathfrak{J} \beta_{j}\right]^{2}\right)}
$$

in $\mathfrak{J} S_{\ell}\left(B^{(\Lambda)}\right)$. Consequently, the monomial (11.3) can only vanish if it is also contained in the right summand $\Pi_{\Lambda} \mathfrak{J} S_{\ell}(B) \mathfrak{R} S_{\ell}\left(B^{(\Lambda)}\right)$.

Assuming that this is the case, we observe that the considered monomial also uniquely arises from the factor

$$
\mathfrak{R} \beta_{2} \cdots \mathfrak{R} \beta_{\ell+1}
$$

in $\mathfrak{J} S_{\ell}(B)$ and the factor

$$
\frac{\mathfrak{J} \beta_{1} \mathfrak{R} \beta_{2} \ldots \mathfrak{R} \beta_{\ell}}{\prod_{j=1}^{J}\left(\left[\mathfrak{R} \beta_{j}\right]^{2}+\left[\mathfrak{J} \beta_{j}\right]^{2}\right)}
$$

in $\mathfrak{R} S_{\ell}\left(B^{(\Lambda)}\right)$ with a completely analogous argumentation. However, this is impossible because the imaginary part $\mathfrak{J} S_{\ell}(B)$ consists only of monomials with an odd number of 'imaginary variables' $\mathfrak{J} \beta_{j}$ and the real part $\mathfrak{R} S_{\ell}\left(B^{(\Lambda)}\right)$ of monomials with an even number. Therefore, the algebraic polynomial in (11.2) is not constantly zero.

(ii) For the remaining case $1 \leq \ell \leq J \leq N-1$, we firstly assume that $2 \ell \geq N-1$ and investigate the monomial

$$
\begin{gathered}
\mathfrak{J} \beta_{1} \mathfrak{R} \beta_{2} \cdots \mathfrak{R} \beta_{N-1-\ell}\left[\mathfrak{R} \beta_{N-\ell}\right]^{2} \cdots\left[\mathfrak{R} \beta_{\ell}\right]^{2} \\
\cdot\left[\mathfrak{R} \beta_{\ell+1}\right]^{3} \cdots\left[\mathfrak{R} \beta_{J}\right]^{3} \mathfrak{R} \beta_{J+1} \cdots \mathfrak{R} \beta_{N-1},
\end{gathered}
$$

where the three last products can be empty. Again, we try to reconstruct the index sets $K$ of the factor from $\mathfrak{R} S_{\ell}(B)$ and $\breve{K}$ from $\mathfrak{J} S_{\ell}\left(B^{(\Lambda)}\right)$.

Considering that the monomial (11.4) consists of $\ell$ variables with indices from 1 to $\ell$, further $\ell$ variables with indices from $N-\ell$ to $N-1$, and the $J-\ell$ 
remaining quadratic variables with indicies from $\ell+1$ to $J$, we observe that the monomial (11.4) has a degree of $2 J$. Consequently, exactly $\ell$ quadratic factors $\left[\mathfrak{R} \beta_{j}\right]^{2}+\left[\mathfrak{J} \beta_{j}\right]^{2}$ of the prefactor $\Pi_{\Lambda}$ have to be canceled with the inverse moduli in $\mathfrak{J} S_{\ell}\left(B^{(\Lambda)}\right)$, which implies that $\breve{K}$ is a subset of $\{1, \ldots, J\}$.

Since the first $N-1-\ell$ variables have exponent one, the corresponding quadratic factors in $\Pi_{\Lambda}$ have to be canceled; so at least the indices from 1 to $N-1-\ell$ must be contained in $\breve{K}$. The only way to generate variables with exponent three is to multiply the corresponding quadratic factor in $\Pi_{\Lambda}$ with a variable from $\mathfrak{R} S_{\ell}(B)$. We can therefore conclude that the index of these variables cannot be in $\breve{K}$, and hence the indicies from $\ell+1$ to $J$ are contained in $K$. Further, the indices from $l+1$ to $N-1$ must also be in $K$ because the index set $\breve{K}$ can only contain indices less than or equal to $J$.

At the moment both index sets comprise $N-1-\ell$ indicies: $K$ the indicies from $\ell+1$ to $N-1$ and $\breve{K}$ the indicies from 1 to $N-1-\ell$. Further, we know that $J-\ell$ quadratic variables with index between $\ell+1$ and $J$ arise from $\Pi_{\Lambda}$. Consequently, the remaining squared variables with indicies from $N-\ell$ to $\ell$ must be contained in both index sets to ensure that $K$ and $\breve{K}$ exactly comprise $\ell$ elements. Now, since both index sets are completely determined, we can see that the monomial (11.4) uniquely arises from the factor

$$
\mathfrak{R} \beta_{N-\ell} \cdots \mathfrak{R} \beta_{N-1}
$$

from $\mathfrak{R} S_{\ell}(B)$ and

$$
\frac{\mathfrak{J} \beta_{1} \mathfrak{R} \beta_{2} \cdots \mathfrak{R} \beta_{\ell}}{\prod_{j=1}^{\ell}\left(\left[\mathfrak{R} \beta_{j}\right]^{2}+\left[\mathfrak{J} \beta_{j}\right]^{2}\right)}
$$

from $\mathfrak{I} S_{\ell}\left(B^{(\Lambda)}\right)$.

Using an analogous argumentation as in (i), the considered monomial (11.4) can only vanish if it is also contained in $\Pi_{\Lambda} \mathfrak{J} S_{\ell}(B) \mathfrak{R} S_{\ell}\left(B^{(\Lambda)}\right)$. However, this would lead to the same factorization of the monomial (11.4) where the factors arise from the imaginary part instead of the real part of $S_{\ell}$ and vice versa. Due to the fact that the real and imaginary part of $S_{\ell}$ consists of factors with even or odd numbers of 'imaginary variables' $\mathfrak{J} \beta_{j}$ respectively, this factorization is invalid, and the algebraic polynomial (11.2) is non-trivial.

It remains to show that the polynomial is also non-trivial for $2 \ell<N-1$. For this purpose, we examine the monomials

$$
\begin{aligned}
\mathfrak{J} \beta_{1} \mathfrak{R} \beta_{2} \cdots \mathfrak{R} \beta_{\ell}\left[\mathfrak{R} \beta_{\ell+1}\right]^{2} \cdots\left[\beta_{N-\ell-1}\right]^{2} \\
\quad \cdot\left[\mathfrak{R} \beta_{N-\ell}\right]^{3} \cdots\left[\mathfrak{R} \beta_{J}\right]^{3} \mathfrak{R} \beta_{J+1} \cdots \mathfrak{R} \beta_{N-1}
\end{aligned}
$$


with the limitation $N-\ell \leq J$ and

$$
\mathfrak{J} \beta_{1} \mathfrak{R} \beta_{2} \cdots \mathfrak{R} \beta_{\ell}\left[\mathfrak{R} \beta_{\ell+1}\right]^{2} \cdots\left[\mathfrak{R} \beta_{J}\right]^{2} \mathfrak{R} \beta_{N-\ell} \cdots \mathfrak{R} \beta_{N-1}
$$

otherwise.

In a similar way as above, the quadratic factors $\left[\mathfrak{R} \beta_{j}\right]^{2}+\left[\mathfrak{J} \beta_{j}\right]^{2}$ of $\Pi_{\Lambda}$ have to be canceled for indices between 1 and $\ell$, which directly implies that the index set $\breve{K}$ must comprise these $\ell$ indicies. Considering the remaining variables with exponent three and one, we can analogously argue that $K$ contains the $\ell$ indicies from $N-\ell$ to $N-1$. Compared with the monomial (11.3), the squared variables in (11.5) and (11.6) here arise from the prefactor $\Pi_{\Lambda}$. Since both index sets $K$ and $\breve{K}$ coincide with the examination above, the monomials have the same unique factorization, and thus the polynomial in (11.2) is not constantly zero.

After this cumbersome case study, the polynomial equation (11.2) is non-trivial for every $\ell$ between 1 and $N-2$ and every non-empty subset $\Lambda$ of the corresponding zero set $B$. Moreover, the algebraic polynomial is non-constant because the considered monomials have a non-vanishing degree. All in all, the corresponding zero sets satisfying the linear equation in the assertion is contained in the zero locus of the algebraic polynomial in (11.2), which completes the proof.

Considering the union of the constructed algebraic varieties in Lemma 11.2 for all possible subsets $\Lambda$, we can conclude that the additional phase information can indeed enforce uniqueness of the reconstruction for almost every discrete-time signal.

Theorem 11.3. Let $x$ be a complex-valued discrete-time signal with normalized support of length $N$, and let $\ell$ be an arbitrary integer between 1 and $N-2$. The phase retrieval problem to recover the signal $x$ from its FOURIER intensity $|\widehat{x}|$ and the two phases $\arg x[N-1]$ and $\arg x[N-1-\ell]$ is almost always uniquely solvable.

Proof. Considering the corresponding zero sets of a discrete-time signal with normalized support as $(2 N-2)$-dimensional vector, we observe in Lemma 11.2 that the zero sets that satisfy the non-uniqueness conditions in Theorem 11.1 for a specific subset $\Lambda$ lie in the zero locus of an algebraic polynomial. Since this polynomial is not constantly zero, the exceptional zero sets are contained in a set with zero LEBESGUE measure, and hence also the union of them. 
Although the constructed union does not only comprise valid zero sets that fulfil the non-uniqueness conditions in Theorem 11.1 since we have neglected the inequality condition in Theorem 11.1 and the circumstance that the corresponding zero sets cannot contain zero elements, the exceptional zero sets of signals without a unique reconstruction also form a set with measure zero as subset of this union. Using VIETA's formulare (Theorem 8.7) and SARD's theorem (Theorem 10.5), we can deduce the assertion as in the proof of Theorem 10.6.

\subsection{Phase of two arbitrary points}

A straightforward generalization of our findings in Section 11.1 is the discretetime phase retrieval problem where the phase of the end point is replaced by the phase of a further inner point of the unknown signal. In other words, we consider the phase retrieval problem to recover an unknown signal $x$ from its Fourier intensity $|\widehat{x}|$ and the phases $\arg x\left[N-1-\ell_{1}\right]$ and $\arg x\left[N-1-\ell_{2}\right]$ of two inner points in the time domain, where $\ell_{1}$ and $\ell_{2}$ are two different integers between 1 and $N-2$. In analogy to Theorem 11.1, we can characterize the corresponding zero sets of signals that cannot be uniquely reconstructed by interpreting the phase conditions as an equation of a straight line.

Theorem 11.4. Let $x$ be a complex-valued discrete-time signal with normalized support of length $N$ and corresponding zero set $B:=\left\{\beta_{1}, \ldots, \beta_{N-1}\right\}$, and let $\ell_{1}$ and $\ell_{2}$ be different integers between 1 and $N-2$. Then the signal $x$ cannot be uniquely recovered from its FOURIER intensity $|\widehat{x}|$ and the two phases $\arg x\left[N-1-\ell_{1}\right]$ and $\arg x\left[N-1-\ell_{2}\right]$ if and only if there exists a non-empty subset $\Lambda$ of $B$, where $\Lambda$ does not contain reflected zero pairs or zeros on the unit circle, such that the corresponding zero set $B$ and the modified zero set $B^{(\Lambda)}$ fulfil

$$
\begin{aligned}
\mathfrak{R}\left[S_{\ell_{1}}(B)\right] \mathfrak{J}\left[\overline{S_{\ell_{2}}\left(B^{(\Lambda)}\right)} S_{\ell_{2}}(B) S_{\ell_{1}}\left(B^{(\Lambda)}\right)\right] \\
\quad-\mathfrak{J}\left[S_{\ell_{1}}(B)\right] \mathfrak{R}\left[\overline{S_{\ell_{2}}\left(B^{(\Lambda)}\right)} S_{\ell_{2}}(B) S_{\ell_{1}}\left(B^{(\Lambda)}\right)\right]=0
\end{aligned}
$$

and further

$$
\begin{aligned}
& \mathfrak{R}\left[S_{\ell_{1}}(B)\right] \mathfrak{R}\left[\overline{S_{\ell_{2}}\left(B^{(\Lambda)}\right)} S_{\ell_{2}}(B) S_{\ell_{1}}\left(B^{(\Lambda)}\right)\right] \\
& \quad+\mathfrak{J}\left[S_{\ell_{1}}(B)\right] \mathfrak{J}\left[\overline{S_{\ell_{2}}\left(B^{(\Lambda)}\right)} S_{\ell_{2}}(B) S_{\ell_{1}}\left(B^{(\Lambda)}\right)\right] \geq 0 .
\end{aligned}
$$


Proof. Again, we assume that the phase retrieval problem to recover $x$ from its FourIER intensity and the phases $\arg x\left[N-1-\ell_{1}\right]$ and $\arg x\left[N-1-\ell_{2}\right]$ in the time domain has a further non-trivial solution $y$. Consequently, we find a subset $\Lambda$ of the corresponding zero set $B$ so that the second solution $y$ corresponds to the modified zero set $B^{(\Lambda)}$, where $\Lambda$ does not contain reflected zero pairs or zeros on the unit circle.

Due to the trivial rotation ambiguity in Proposition 2.1, we can always rotate the second signal $y$ such that the phases arg $x\left[N-1-\ell_{2}\right]$ and $\arg y\left[N-1-\ell_{2}\right]$ coincide. In other words, we rotate the non-trivial ambiguity $y$ by the multiplication with the unimodular factor

$$
\mathrm{e}^{\mathrm{i}\left(\arg x\left[N-1-\ell_{2}\right]-\arg y\left[N-1-\ell_{2}\right]\right)}=\frac{\overline{y\left[N-1-\ell_{2}\right]}}{\left|y\left[N-1-\ell_{2}\right]\right|} \frac{x\left[N-1-\ell_{2}\right]}{\left|x\left[N-1-\ell_{2}\right]\right|}
$$

whenever $\left|y\left[N-1-\ell_{2}\right]\right|$ and $\left|x\left[N-1-\ell_{2}\right]\right|$ are non-zero. If one of these moduli is zero, then the linear equation and the inequality condition in the assertion are obviously fulfilled.

Next, we investigate the equality of the remaining given phase in the time domain. Bearing the additional rotation of the ambiguity $y$ in mind, we obtain the restriction

$$
\begin{aligned}
\arg x\left[N-1-\ell_{1}\right] & =\arg \left(\frac{\overline{y\left[N-1-\ell_{2}\right]}}{\left|y\left[N-1-\ell_{2}\right]\right|} \frac{x\left[N-1-\ell_{2}\right]}{\left|x\left[N-1-\ell_{2}\right]\right|} y\left[N-1-\ell_{1}\right]\right) \\
& =\arg \left(\overline{y\left[N-1-\ell_{2}\right]} x\left[N-1-\ell_{2}\right] y\left[N-1-\ell_{1}\right]\right)
\end{aligned}
$$

since the multiplication with a positive real factor does not change the phase of a complex number.

Again, the components of the signal $x$ with normalized support are given by

$$
x[N-1-\ell]=(-1)^{\ell} \mathrm{e}^{\mathrm{i} \alpha} \sqrt{|a[N-1]| \prod_{j=1}^{N-1}\left|\beta_{j}\right|^{-1}} \cdot S_{\ell}(B)
$$

as discussed in Section 8. With an analogous representation of the components of the further solution $y$, we can simplify the considered phase restriction to

$$
\arg \left((-1)^{\ell_{1}} \mathrm{e}^{\mathrm{i} \alpha} S_{\ell_{1}}(B)\right)=\arg \left((-1)^{\ell_{1}} \mathrm{e}^{\mathrm{i} \alpha} \overline{S_{\ell_{2}}\left(B^{(\Lambda)}\right)} S_{\ell_{2}}(B) S_{\ell_{1}}\left(B^{(\Lambda)}\right)\right),
$$

where we already neglect the real positive factors in the representations of the signal values. Finally, this equation is equivalent to

$$
\arg \left(S_{\ell_{1}}(B)\right)=\arg \left(\overline{S_{\ell_{2}}\left(B^{(\Lambda)}\right)} S_{\ell_{2}}(B) S_{\ell_{1}}\left(B^{(\Lambda)}\right)\right)
$$

since the prefactor $(-1)^{\ell_{1}} \mathrm{e}^{\mathrm{i} \alpha}$ of the arguments on both sides coincide. 
Following the procedure in the proof of Theorem 11.1, the complex numbers

$$
S_{\ell_{1}}(B) \text { and } \overline{S_{\ell_{2}}\left(B^{(\Lambda)}\right)} S_{\ell_{2}}(B) S_{\ell_{1}}\left(B^{(\Lambda)}\right)
$$

have to lie on the same ray starting from the origin in the complex plane, which results in the linear equation and the inequality condition of the assertion.

In some special cases, it is never possible to recover the wanted signal without any ambiguities - trivial and non-trivial. For instance, if we try to recover a discrete-time signal $x$ with normalized support from its FourIER intensity and the two phases $\arg x[\ell]$ and $\arg x[N-1-\ell]$, where $\ell$ is an integer between 1 and $N-2$, then a rotated version

$$
y:=\mathrm{e}^{\mathrm{i}(\arg x[\ell]+\arg x[N-1-\ell])} \overline{x[N-1-\cdot]}
$$

of the conjugated and reflected signal $x$ always solves the considered phase retrieval problem too. More detailed, the corresponding phases of $y$ are obviously given by

$$
\arg y[\ell]=\arg x[\ell]+\arg x[N-1-\ell]-\arg x[N-1-\ell]
$$

and

$$
\arg y[N-1-\ell]=\arg x[\ell]+\arg x[N-1-\ell]-\arg x[\ell],
$$

which implies that $y$ really is an ambiguity. Consequently, if the corresponding zero set does not only consists of reflected pairs or zeros on the unit circle, we always find a subset $\Lambda$ - the complete zero set - so that Theorem 11.4 holds true, and the considered signal cannot be recovered uniquely.

In order to eliminate this special case, we assume that the second solution $y$ in the proof of Theorem 11.4 is not a rotation of the conjugated and reflected original signal $x$. In other words, we assume that $\Lambda$ is a proper subset that cannot be extended to the complete zero set by adding reflected zeros or zeros on the unit circle. Adapting the proof of Theorem 11.4 in this manner, we have the following slightly weaker statement.

Corollary 11.5. Let $x$ be a complex-valued discrete-time signal with normalized support of length $N$ and corresponding zero set $B:=\left\{\beta_{1}, \ldots, \beta_{N-1}\right\}$, and let $\ell$ be an arbitrary integer between 1 and $N-2$. Then the signal $x$ cannot be uniquely recovered (up to conjugate reflections) from its FOURIER intensity $|\widehat{x}|$ and the two phases $\arg x[\ell]$ and $\arg x[N-1-\ell]$ if and only if there exists a non-empty proper subset $\Lambda$ of the corresponding zero set $B$, where $\Lambda$ does not contain reflected zero 
pairs or zeros on the unit circle and cannot be extended to the complete set $B$ by adding zeros of this kind, such that the corresponding zero set $B$ and the modified zero set $B^{(\Lambda)}$ fulfil

$$
\begin{aligned}
\mathfrak{R}\left[S_{\ell}(B)\right] \mathfrak{J}\left[\overline{S_{N-1-\ell}\left(B^{(\Lambda)}\right)} S_{N-1-\ell}(B) S_{\ell}\left(B^{(\Lambda)}\right)\right] \\
\quad-\mathfrak{J}\left[S_{\ell}(B)\right] \mathfrak{R}\left[\overline{S_{N-1-\ell}\left(B^{(\Lambda)}\right)} S_{N-1-\ell}(B) S_{\ell}\left(B^{(\Lambda)}\right)\right]=0
\end{aligned}
$$

and further

$$
\begin{aligned}
& \mathfrak{R}\left[S_{\ell_{1}}(B)\right] \mathfrak{R}\left[\overline{S_{\ell_{2}}\left(B^{(\Lambda)}\right)} S_{\ell_{2}}(B) S_{\ell_{1}}\left(B^{(\Lambda)}\right)\right] \\
& \quad+\mathfrak{J}\left[S_{\ell_{1}}(B)\right] \mathfrak{J}\left[\overline{S_{\ell_{2}}\left(B^{(\Lambda)}\right)} S_{\ell_{2}}(B) S_{\ell_{1}}\left(B^{(\Lambda)}\right)\right] \geq 0 .
\end{aligned}
$$

As many times before, we interpret the corresponding zero set $\left\{\beta_{1}, \ldots, \beta_{N-1}\right\}$ of a discrete-time signal with normalized support as the $(2 N-2)$-dimensional vector

$$
\left(\mathfrak{R} \beta_{1}, \mathfrak{J} \beta_{1}, \ldots, \mathfrak{R} \beta_{N-1}, \mathfrak{J} \beta_{N-1}\right)^{\mathrm{T}}
$$

and show that the zero sets of signals that cannot be uniquely recovered are contained in a union of suitable algebraic varieties. Again, we only investigate the linear equation of Theorem 11.4 and Corollary 11.5, and neglect the additional inequality restriction. In this manner, we can show the following result.

Lemma 11.6. If the corresponding zero set $B:=\left\{\beta_{1}, \ldots, \beta_{N-1}\right\}$ of a discrete-time signal is considered as a real $(2 N-2)$-dimensional vector, then the zero sets satisfying

$$
\begin{aligned}
& \mathfrak{R}\left[S_{\ell_{1}}(B)\right] \mathfrak{J}\left[\overline{S_{\ell_{2}}\left(B^{(\Lambda)}\right)} S_{\ell_{2}}(B) S_{\ell_{1}}\left(B^{(\Lambda)}\right)\right] \\
& \quad-\mathfrak{J}\left[S_{\ell_{1}}(B)\right] \mathfrak{R}\left[\overline{S_{\ell_{2}}\left(B^{(\Lambda)}\right)} S_{\ell_{2}}(B) S_{\ell_{1}}\left(B^{(\Lambda)}\right)\right]=0
\end{aligned}
$$

lie in the zero locus of a non-constant polynomial for every non-empty subset $\Lambda$ of $B$ and different $\ell_{1}$ and $\ell_{2}$ between 1 and $N-2$ or, when $\ell_{1}+\ell_{2}=N-1$, for every proper subset $\Lambda$.

Proof. Lemma 11.6 can be interpreted as a generalization of Lemma 11.2, and we can prove the assertion in the same manner. Again, we write the complex 
variables $\beta_{j}$ of equation (11.7) in their algebraic form $\mathfrak{R} \beta_{j}+\mathrm{i} \mathfrak{J} \beta_{j}$. In order to cancel the inverse moduli of the reflected zeros in $S_{\ell_{1}}\left(B^{(\Lambda)}\right)$ and $S_{\ell_{2}}\left(B^{(\Lambda)}\right)$, we furthermore multiply the equation of a straight line (11.7) with

$$
\Pi_{\Lambda}:=\prod_{\beta_{j} \in \Lambda}\left(\left[\mathfrak{R} \beta_{j}\right]^{2}+\left[\mathfrak{J} \beta_{j}\right]^{2}\right)^{2}
$$

since each reflected zero can occur at most twice. In this manner, we obtain the equivalent restriction

$$
\begin{aligned}
\Pi_{\Lambda}(\mathfrak{R}[ & \left.S_{\ell_{1}}(B)\right] \mathfrak{J}\left[\overline{S_{\ell_{2}}\left(B^{(\Lambda)}\right)} S_{\ell_{2}}(B) S_{\ell_{1}}\left(B^{(\Lambda)}\right)\right] \\
& \left.-\mathfrak{J}\left[S_{\ell_{1}}(B)\right] \mathfrak{R}\left[\overline{S_{\ell_{2}}\left(B^{(\Lambda)}\right)} S_{\ell_{2}}(B) S_{\ell_{1}}\left(B^{(\Lambda)}\right)\right]\right)=0,
\end{aligned}
$$

whose left-hand side can be continuously extended to an algebraic polynomial in the real variables $\mathfrak{R} \beta_{j}$ and $\mathfrak{J} \beta_{j}$.

It remains the question whether the polynomial in (11.8) vanishes everywhere or not. Again, to simplify the investigation of this issue, we assume without loss of generality that the fixed subset $\Lambda$ contains the first $J$ corresponding zeros $\left\{\beta_{1}, \ldots, \beta_{J}\right\}$, and that $\ell_{1}>\ell_{2}$. Now, we follow the approach in the proof of Lemma 11.2 in a slightly modified form. This means that we pick one single monomial in the polynomial

$$
\Pi_{\Lambda} \mathfrak{I}\left[S_{\ell_{1}}(B)\right] \mathfrak{R}\left[\overline{S_{\ell_{2}}\left(B^{(\Lambda)}\right)} S_{\ell_{2}}(B) S_{\ell_{1}}\left(B^{(\Lambda)}\right)\right]
$$

and show that the considered monomial cannot vanish within this polynomial. Afterwards we show that the chosen monomial cannot occur in the other polynomial

$$
\Pi_{\Lambda} \mathfrak{R}\left[S_{\ell_{1}}(B)\right] \mathfrak{J}\left[\overline{S_{\ell_{2}}\left(B^{(\Lambda)}\right)} S_{\ell_{2}}(B) S_{\ell_{1}}\left(B^{(\Lambda)}\right)\right]
$$

so the polynomial in (11.8) contains at least one non-vanishing monomial and hence is non-trivial.

In analogy to the proof of Lemma 11.2, we only consider monomials where the real part $\mathfrak{R} \beta_{j}$ and the imaginary part $\mathfrak{J} \beta_{j}$ of one corresponding zero $\beta_{j}$ do not occur simultaneously. To determine the factorization of the monomials with respect to the four occurring elementary symmetric functions, it is therefore enough to determine the index sets $K_{\ell_{1}}, \breve{K}_{\ell_{1}}, K_{\ell_{2}}$, and $\breve{K}_{\ell_{2}}$ of the real indeterminates in the factors $S_{\ell_{1}}(B), S_{\ell_{1}}\left(B^{(\Lambda)}\right), S_{\ell_{2}}(B)$, and $S_{\ell_{2}}\left(B^{(\Lambda)}\right)$ respectively. Unfortunately, the polynomial in (11.8) depends on $\ell_{1}, \ell_{2}$, and $J$, which leads to the following cumbersome case study. 
We distinguish three main cases: $J \leq \ell_{2}<\ell_{1}, \ell_{2}<J \leq \ell_{1}$, and $\ell_{2}<\ell_{1} \leq J$. For this cases, we now consider a specific monomial and show that the occurring variables can be uniquely assigned to the elementary symmetric functions in (11.9). Although each of the main cases is based on one monomial, we need different argumentations depending on $\ell_{1}$ and $\ell_{2}$ to show that the factorization indeed is unique. More detailed, we will divide the second case $\ell_{2}<J \leq \ell_{1}$ in the two subcases $2 \ell_{2} \leq J$ and $2 \ell_{2}>J$. For the last main case $\ell_{2}<\ell_{1} \leq J$, we further distinguish between $2 \ell_{2}<2 \ell_{1} \leq J, 2 \ell_{2} \leq J<2 \ell_{1}$, and $J<2 \ell_{2}<2 \ell_{1}$.

The crucial point of the following argumentation is the case $\ell_{2}<\ell_{1} \leq J$ with $2 \ell_{2} \leq J<2 \ell_{1}$. We will see that in the special case $\ell_{1}+\ell_{2}=J=N-1$, which is not covered by the assertion, the polynomial on the left-hand side of (11.8) becomes trivial. To overcome this difficulties and complete our case study, we will here consider three further subcases: $\ell_{1}+\ell_{2}<J, \ell_{1}+\ell_{2}=J$, and $\ell_{1}+\ell_{2}>J$.

(i) Firstly, we assume that $\ell_{1}>\ell_{2} \geq J$, and investigate the monomial

$$
\begin{aligned}
& {\left[\mathfrak{R} \beta_{1}\right]^{2}\left[\mathfrak{R} \beta_{2}\right]^{4} } \cdots\left[\mathfrak{R} \beta_{\ell_{2}}\right]^{4}\left[\mathfrak{R} \beta_{\ell_{2}+1}\right]^{3} \\
& \cdot\left[\mathfrak{R} \beta_{\ell_{2}+2}\right]^{2} \cdots\left[\mathfrak{R} \beta_{\ell_{1}}\right]^{2} \mathfrak{J} \beta_{\ell_{1}+1}
\end{aligned}
$$

where the product over the squared variables with indices from $\ell_{2}+2$ to $\ell_{1}$ can be empty. A brief computation shows that the degree of this monomial is $2\left(\ell_{1}+\ell_{2}\right)$. Consequently, the considered monomial cannot contain any quadratic factor $\left[\mathfrak{R} \beta_{j}\right]^{2}$ or $\left[\mathfrak{J} \beta_{j}\right]^{2}$ from the prefactor $\Pi_{\Lambda}$, which implies that the complete prefactor $\Pi_{\Lambda}$ and the inverse moduli from $S_{\ell_{1}}\left(B^{(\Lambda)}\right)$ and $S_{\ell_{2}}\left(B^{(\Lambda)}\right)$ must cancel each other. Therefore, both index sets $\breve{K}_{\ell_{1}}$ and $\breve{K}_{\ell_{2}}$ have to contain the indicies from 1 to $J$.

With this preliminary observation, we can uniquely determine all four index sets. As seen above, the index one is in $\breve{K}_{\ell_{1}}$ and $\breve{K}_{\ell_{2}}$. Further, the indicies from 2 to $\ell_{2}$ have to be contained in all index sets because the corresponding variables have the exponent four. Since the first index set $\breve{K}_{\ell_{2}}$ already comprises $\ell_{2}$ indices, it follows that the index $\ell_{2}+1$ is contained in the three remaining index sets. Now, the indices from $\ell_{2}+2$ to $\ell_{1}$ of the quadratic variables have to be assigned to $K_{\ell_{1}}$ and $\breve{K}_{\ell_{1}}$ due to the fact that $K_{\ell_{2}}$ also comprises $\ell_{2}$ indices. The last remaining index of $\mathfrak{J} \beta_{\ell_{1}+1}$ must be contained in $K_{\ell_{1}}$ because we have already completely determined the other index sets.

In the end, considering the four index sets $K_{\ell_{1}}, \breve{K}_{\ell_{1}}, K_{\ell_{2}}$, and $\breve{K}_{\ell_{2}}$, we can deduce that the monomial (11.11) uniquely arises from

$$
\mathfrak{R} \beta_{2} \cdots \mathfrak{R} \beta_{\ell_{1}} \mathfrak{J} \beta_{\ell_{1}+1}
$$


in $\mathfrak{J}\left[S_{\ell_{1}}(B)\right]$ and

$$
\frac{\left[\mathfrak{R} \beta_{1}\right]^{2}\left[\mathfrak{R} \beta_{2}\right]^{3} \cdots\left[\mathfrak{R} \beta_{k}\right]^{3}\left[\mathfrak{R} \beta_{k+1}\right]^{2} \mathfrak{R} \beta_{k+2} \cdots \mathfrak{R} \beta_{\ell}}{\prod_{j=1}^{J}\left(\left[\mathfrak{R} \beta_{j}\right]^{2}+\left[\mathfrak{J} \beta_{j}\right]^{2}\right)^{2}}
$$

in $\mathfrak{R}\left[\overline{S_{\ell_{2}}\left(B^{(\Lambda)}\right)} S_{\ell_{2}}(B) S_{\ell_{1}}\left(B^{(\Lambda)}\right)\right]$. Since this factorization is unique, the considered monomial (11.11) cannot vanish within the polynomial (11.9).

As discussed in the proof of Lemma 11.2, the monomials in the real part of $S_{\ell_{1}}(B)$ contain an even number of 'imaginary variables' $\mathfrak{J} \beta_{j}$, whereas the monomials in the imaginary part contain an odd number. This observation can be generalized to an arbitrary product of corresponding zeros, where the particular zeros can appear with higher multiplicity. The same observation also holds true for the real and imaginary parts of

$$
\overline{S_{\ell_{2}}\left(B^{(\Lambda)}\right)} S_{\ell_{2}}(B) S_{\ell_{1}}\left(B^{(\Lambda)}\right) .
$$

Using this statement, we can conclude that the monomial (11.11) cannot be contained in the left polynomial in (11.8) because the factor

$$
\mathfrak{R} \beta_{2} \cdots \mathfrak{R} \beta_{\ell_{1}} \mathfrak{J} \beta_{\ell_{1}+1}
$$

is not a part of $\mathfrak{R}\left[S_{\ell_{1}}(B)\right]$ and also

$$
\frac{\left[\mathfrak{R} \beta_{1}\right]^{2}\left[\mathfrak{R} \beta_{2}\right]^{3} \cdots\left[\mathfrak{R} \beta_{k}\right]^{3}\left[\mathfrak{R} \beta_{k+1}\right]^{2} \mathfrak{R} \beta_{k+2} \cdots \mathfrak{R} \beta_{\ell}}{\prod_{j=1}^{J}\left(\left[\mathfrak{R} \beta_{j}\right]^{2}+\left[\mathfrak{J} \beta_{j}\right]^{2}\right)^{2}}
$$

cannot occur in $\mathfrak{J}\left[\overline{S_{\ell_{2}}\left(B^{(\Lambda)}\right)} S_{\ell_{2}}(B) S_{\ell_{1}}\left(B^{(\Lambda)}\right)\right]$.

Finally, the polynomial in (11.8) contains at least the monomial (11.11), which implies that it cannot be constantly zero in this specific case. In order to show that the polynomial equation (11.8) is non-trivial for the remaining cases, we can use a similar argumentation. Therefore, we confine ourselves to the determination of the index sets $K_{\ell_{1}}, \breve{K}_{\ell_{1}}, K_{\ell_{2}}$, and $\breve{K}_{\ell_{2}}$ for a suitable monomial.

(ii) For $\ell_{1} \geq J>\ell_{2}$ with the additional restriction that $2 \ell_{2} \leq J$, we consider the monomial

$$
\begin{aligned}
& {\left[\mathfrak{R} \beta_{1}\right]^{2}\left[\mathfrak{R} \beta_{2}\right]^{3} \cdots\left[\mathfrak{R} \beta_{\ell_{2}}\right]^{3}\left[\mathfrak{R} \beta_{\ell_{2}+1}\right]^{4} \cdots\left[\mathfrak{R} \beta_{J-\ell_{2}}\right]^{4}} \\
& \cdot\left[\mathfrak{R} \beta_{J-\ell_{2}+1}\right]^{5} \cdots\left[\mathfrak{R} \beta_{J}\right]^{5}\left[\mathfrak{R} \beta_{J+1}\right]^{2} \cdots\left[\mathfrak{R} \beta_{\ell_{1}}\right]^{2} \mathfrak{J} \beta_{\ell_{1}+1},
\end{aligned}
$$


where the product over the variables with exponent four can be empty. To determine the representation of this monomial within the left polynomial in (11.8), we examine the influence of the prefactor $\Pi_{\Lambda}$ on a specific monomial of this polynomial. If the squared modulus $\left[\mathfrak{R} \beta_{j}\right]^{2}+\left[\mathfrak{J} \beta_{j}\right]^{2}$ in $\Pi_{\Lambda}$ cancels with the real or imaginary part of a reflected zero, then only a linear factor $\mathfrak{R} \beta_{j}$ or $\mathfrak{J} \beta_{j}$ arises, otherwise the squared modulus in $\Pi_{\Lambda}$ yields a quadratic factor $\left[\mathfrak{R} \beta_{j}\right]^{2}$ or $\left[\mathfrak{J} \beta_{j}\right]^{2}$.

Here the variable $\mathfrak{R} \beta_{1}$ appears only with multiplicity two; so both of the corresponding quadratic factors $\left[\mathfrak{R} \beta_{1}\right]^{2}$ or $\left[\mathfrak{J} \beta_{1}\right]^{2}$ in $\Pi_{\Lambda}$ must be canceled, which implies that both index sets $\breve{K}_{\ell_{1}}$ and $\breve{K}_{\ell_{2}}$ contain the index one. Considering that only $\ell$ different variables remain, we can immediately deduce that $K_{\ell_{1}}$ comprises the $\ell_{1}$ indices from 2 to $\ell_{1}+1$. With exactly the same argument, the index set $\breve{K}_{\ell_{1}}$ now contains the indices between 1 and $\ell_{1}$. Hence, the index sets $K_{\ell_{1}}$ and $\breve{K}_{\ell_{1}}$ are completely determined, and the remaining unassigned variables of the monomial (11.12) are given by

$$
\mathfrak{R} \beta_{2} \cdots \mathfrak{R} \beta_{\ell_{2}}\left[\mathfrak{R} \beta_{\ell_{2}+1}\right]^{2} \cdots\left[\mathfrak{R} \beta_{J-\ell_{2}}\right]^{2}\left[\mathfrak{R} \beta_{J-\ell_{2}+1}\right]^{3} \cdots\left[\mathfrak{R} \beta_{J}\right]^{3} .
$$

Obviously, the remaining simple variables cannot arise from the prefactor $\Pi_{\Lambda}$. Consequently, the corresponding quadratic factors from $\Pi_{\Lambda}$ have to be canceled, which implies that the indices between 2 and $\ell_{2}$ are in the index set $\breve{K}_{\ell_{2}}$. Now, the variables with exponent three can only arise from a quadratic factor in $\Pi_{\Lambda}$ and an additional variable with multiplicity one. In other words, the $K_{\ell_{2}}$ have to comprise the $\ell_{2}$ indices from $J-\ell_{2}+1$ to $J$.

All in all, we uniquely determine the four index sets $K_{\ell_{1}}, \breve{K}_{\ell_{1}}, K_{\ell_{2}}$, and $\breve{K}_{\ell_{2}}$, and show that the monomial (11.12) contains the factor

$$
\mathfrak{R} \beta_{2} \cdots \mathfrak{R} \beta_{\ell_{1}} \mathfrak{J} \beta_{\ell_{1}+1}
$$

from $\mathfrak{J}\left[S_{\ell_{1}}(B)\right]$. With an analogous argumentation as in (i), this factor cannot arise from $\mathfrak{R}\left[S_{\ell_{1}}(B)\right]$, which finally implies that the polynomial equation (11.8) is non-trivial.

(iii) In the case that $\ell_{1} \geq J>\ell_{2}$, but this time with $2 \ell_{2}>J$, we start with the monomial

$$
\begin{aligned}
{\left[\mathfrak{R} \beta_{1}\right]^{2}\left[\mathfrak{R} \beta_{2}\right]^{3} \cdots\left[\mathfrak{R} \beta_{J-\ell_{2}}\right]^{3}\left[\mathfrak{R} \beta_{J-\ell_{2}+1}\right]^{4} \cdots\left[\mathfrak{R} \beta_{\ell_{2}}\right]^{4} } \\
\cdot\left[\mathfrak{R} \beta_{\ell_{2}+1}\right]^{5} \cdots\left[\mathfrak{R} \beta_{J}\right]^{5}\left[\mathfrak{R} \beta_{J+1}\right]^{2} \cdots\left[\mathfrak{R} \beta_{\ell_{1}}\right]^{2} \mathfrak{J} \beta_{\ell_{1}+1} .
\end{aligned}
$$

In exactly the same manner as in (ii), we can conclude that $K_{\ell_{1}}$ comprises 
the indices from 2 to $\ell_{1}+1$, and $\breve{K}_{\ell_{1}}$ the indices from 1 to $\ell_{1}$. Furthermore, the index set $\breve{K}_{\ell_{2}}$ contains at least the indices from 1 to $J-\ell_{2}$, and $K_{\ell_{2}}$ at least the indices from $\ell_{2}+1$ to $J$.

Since $\ell_{2}>\mathrm{J} / 2$, both index sets $K_{\ell_{2}}$ and $\breve{K}_{\ell_{2}}$ are not completely determined at this moment. In order to assign the remaining variables, we observe that $\breve{K}_{\ell_{2}}$ cannot contain the indices of the variables with exponent five because these variables consist of a quadratic factor from $\Pi_{\Lambda}$ as discussed in (ii). Hence, $K_{\ell_{1}}$ and $\breve{K}_{\ell_{2}}$ can only be extended to a set of $\ell_{2}$ elements by the indices of the remaining unassigned variables in

$$
\left[\Re \beta_{J-\ell_{2}+1}\right]^{2} \cdots\left[\Re \beta_{\ell_{2}}\right]^{2} .
$$

Consequently, the index set $K_{\ell_{2}}$ consists of the indices from $J-\ell_{2}+1$ to $J$, and the index set $\breve{K}_{\ell_{2}}$ of the indices from 1 to $\ell_{2}$.

In the end, the index sets $K_{\ell_{1}}, \breve{K}_{\ell_{1}}, K_{\ell_{2}}$, and $\breve{K}_{\ell_{2}}$ are uniquely determined, and the monomial (11.13) arises from the factor

$$
\mathfrak{R} \beta_{2} \cdots \mathfrak{R} \beta_{\ell_{1}} \mathfrak{J} \beta_{\ell_{1}+1}
$$

in $\mathfrak{J}\left[S_{\ell_{1}}(B)\right]$. With a similar argument as in (i), this factor cannot be in $\mathfrak{R}\left[S_{\ell_{1}}(B)\right]$, which implies that the polynomial in (11.8) is not constantly zero.

(iv) Now, let us assume that $\ell_{2}<\ell_{1}<J$ with $2 \ell_{2}<2 \ell_{1} \leq J$. Here we consider the specific monomial

$$
\begin{gathered}
{\left[\mathfrak{R} \beta_{1}\right]^{2} \cdots\left[\mathfrak{R} \beta_{\ell_{2}}\right]^{2}\left[\mathfrak{R} \beta_{\ell_{2}+1}\right]^{3} \cdots\left[\mathfrak{R} \beta_{\ell_{1}}\right]^{3}\left[\mathfrak{R} \beta_{\ell_{1}+1}\right]^{4} \cdots\left[\mathfrak{R} \beta_{J-\ell_{1}}\right]^{4}} \\
\cdot\left[\mathfrak{J} \beta_{J-\ell_{1}+1}\right]^{5}\left[\mathfrak{R} \beta_{J-\ell_{1}+2}\right]^{5} \cdots\left[\mathfrak{R} \beta_{J-\ell_{2}}\right]^{5}\left[\mathfrak{R} \beta_{J-\ell_{2}+1}\right]^{6} \cdots\left[\mathfrak{R} \beta_{J}\right]^{6},
\end{gathered}
$$

where the products of the real part variables with exponent four or five can be empty.

Using the observation in (ii) that variables with exponent two can only arise if both corresponding quadratic factors in $\Pi_{\Lambda}$ are canceled with the inverse modulus of a reflected zero, we can immediately deduce that $\breve{K}_{\ell_{1}}$ and $\breve{K}_{\ell_{2}}$ comprise the indices from 1 to $\ell_{2}$. Since the remaining variables arise at least from one quadratic factor of $\Pi_{\Lambda}$, the indices of the variables with exponent three have to be in $\breve{K}_{\ell_{1}}$; so the set $\breve{K}_{\ell_{1}}$ comprises the indices from 1 to $\ell_{1}$. Conversely, the variables with exponent five and six have to consist of two quadratic factors from $\Pi_{\Lambda}$. Consequently, $K_{\ell_{1}}$ and $K_{\ell_{2}}$ must contain the indices between $J-\ell_{2}+1$ and $J$. Since the index set $K_{\ell_{2}}$ already comprises $\ell_{2}$ elements, the indices of the variables with exponent five have to be in $K_{\ell_{1}}$; therefore the set $K_{\ell_{1}}$ contains the indices from $J-\ell_{1}+1$ to $J$. 
Finally, we can conclude that the monomial (11.14) arises from the factor

$$
\mathfrak{J} \beta_{J-\ell_{1}+1} \mathfrak{R} \beta_{J-\ell_{1}+2} \cdots \mathfrak{R} \beta_{J}
$$

in $\mathfrak{J}\left[S_{\ell_{1}}(B)\right]$. In analogy to the observations in (iv), this factor cannot occur in $\mathfrak{R}\left[S_{\ell_{1}}(B)\right]$, which yields that the polynomial equation (11.8) is nontrivial.

(v) Next, we assume that $\ell_{2}<\ell_{1}<J$ with $2 \ell_{2} \leq J<2 \ell_{1}$. Further, we assume that the additional restriction $\ell_{1}+\ell_{2}<J$ is fulfilled. Here we look at the monomial

$$
\begin{array}{r}
{\left[\mathfrak{R} \beta_{1}\right]^{2} \cdots\left[\mathfrak{R} \beta_{\ell_{2}}\right]^{2}\left[\mathfrak{R} \beta_{\ell_{2}+1}\right]^{3} \cdots\left[\mathfrak{R} \beta_{J-\ell_{1}}\right]^{3}\left[\mathfrak{R} \beta_{J-\ell_{1}+1}\right]^{4} \cdots\left[\mathfrak{R} \beta_{\ell_{1}}\right]^{4}} \\
\cdot\left[\mathfrak{J} \beta_{\ell_{1}+1}\right]^{5}\left[\mathfrak{R} \beta_{\ell_{1}+2}\right]^{5} \cdots\left[\mathfrak{R} \beta_{J-\ell_{2}}\right]^{5}\left[\mathfrak{R} \beta_{J-\ell_{2}+1}\right]^{6} \cdots\left[\mathfrak{R} \beta_{J}\right]^{6},
\end{array}
$$

where the product over the real part variables with exponent five can be empty. With the same argumentation as in (iv), we obtain that $\breve{K}_{\ell_{2}}$ contains the indices from 1 to $\ell_{2}$, and $K_{\ell_{2}}$ the indices from $J-\ell_{2}+1$ to $J$. Moreover, we know that $\breve{K}_{\ell_{1}}$ comprises the indices from 1 to $J-\ell_{1}$, and $K_{\ell_{1}}$ the indices from $\ell_{1}+1$ to $J$.

In order to determine the missing $2 \ell_{1}-J$ indices in $K_{\ell_{1}}$ and $\breve{K}_{\ell_{1}}$, we consider the remaining unassigned variabels in the reduced monomial

$$
\left[\mathfrak{R} \beta_{J-\ell_{1}+1}\right]^{4} \cdots\left[\mathfrak{R} \beta_{\ell_{1}}\right]^{4} .
$$

Since only $2 \ell_{1}-J$ variables are left, we can conclude that these indicies are contained in both sets $K_{\ell_{1}}$ and $\breve{K}_{\ell_{1}}$. Hence, all index sets $K_{\ell_{1}}, \breve{K}_{\ell_{1}}, K_{\ell_{2}}$, and $\breve{K}_{\ell_{2}}$ can be determined without any ambiguity.

In this specific case, the monomial (11.15) arises from the factor

$$
\mathfrak{R} \beta_{J-\ell_{1}+1} \cdots \mathfrak{R} \beta_{\ell_{1}} \mathfrak{J} \beta_{\ell_{1}+1} \mathfrak{R} \beta_{\ell_{1}+2} \cdots \mathfrak{R} \beta_{J}
$$

in $\mathfrak{J}\left[S_{\ell_{1}}(B)\right]$. As before, this factor cannot arise from $\mathfrak{R}\left[S_{\ell_{1}}(B)\right]$, which shows that the polynomial in (11.8) cannot be trivial.

(vi) Starting from $\ell_{2}<\ell_{1}<J$ with $2 \ell_{2} \leq J<2 \ell_{1}$, we investigate the case where $\ell_{1}+\ell_{2}=J$. Initially, we assume that $J<N-1$ and consider the monomial

$$
\begin{aligned}
& {\left[\mathfrak{R} \beta_{1}\right]^{2} \cdots\left[\left[\mathfrak{R} \beta_{\ell_{2}}\right]^{2}\left[\mathfrak{R} \beta_{\ell_{2}+1}\right]^{3}\right.} {\left[\mathfrak{R} \beta_{\ell_{2}+2}\right]^{4} \cdots\left[\mathfrak{R} \beta_{\ell_{1}}\right]^{4} } \\
& \cdot\left[\mathfrak{R} \beta_{\ell_{1}+1}\right]^{6} \cdots\left[\mathfrak{R} \beta_{J}\right]^{6} \mathfrak{J} \beta_{J+1} .
\end{aligned}
$$


Using a similar argumentation as in (iv), we obtain that $\breve{K}_{\ell_{2}}$ contains the indices from 1 to $\ell_{2}$, and $\breve{K}_{\ell_{1}}$ the indices up to $\ell_{2}+1$. In particular, we know that $\left[\mathfrak{R} \beta_{\ell_{2}+1}\right]^{3}$ arises from a quadratic factor in $\Pi_{\Lambda}$.

Now, on the one hand, only $\ell_{1}$ unassigned variables remain, which implies that $K_{\ell_{1}}$ has to comprise the indices between $\ell_{2}+2$ and $J+1$. On the other hand, $K_{\ell_{2}}$ contains the indices from $\ell_{1}+1$ to $J$ because $\breve{K}_{\ell_{2}}$ contains only indices less than $\ell_{1}+1$, and hence the variables with exponent six have to arise from two quadratic factors in $\Pi_{\Lambda}$ and two further variables not reflected at the unit circle. All in all, we determine the three index sets $K_{\ell_{1}}, K_{\ell_{2}}$, and $\breve{K}_{\ell_{2}}$. The remaining unassigned variables of the original monomial (11.16) are given by

$$
\left[\mathfrak{R} \beta_{\ell_{2}+2}\right]^{3} \cdots\left[\mathfrak{R} \beta_{\ell_{1}}\right]^{3} .
$$

Since exactly $\ell_{1}-\ell_{2}-1$ elements of $\breve{K}_{\ell_{1}}$ are missing, the index set $\breve{K}_{\ell_{1}}$ has to comprise the remaining indices of these unassigned variables.

Finally, the monomial (11.16) uniquely arises from the factor

$$
\mathfrak{R} \beta_{\ell_{2}+2} \cdots \mathfrak{R} \beta_{J} \mathfrak{J} \beta_{J+1}
$$

in $\mathfrak{J}\left[S_{\ell_{1}}(B)\right]$, and the polynomial on the left-hand side of (11.8) is nontrivial. Obviously, the whole observation cannot be done when $J$ is equal to $N-1$ because the variable $\mathfrak{J} \beta_{N}$ is not defined. However, if $J$ is equal to $N-1$, then the subset $\Lambda$ comprises the whole corresponding zero set. Together with $\ell_{1}+\ell_{2}=N-1$, this is exactly the case which is not covered by the assertion.

(vii) Like the assumptions in (v) and (vi), we assume that $\ell_{2}<\ell_{1}<J$ with $2 \ell_{2} \leq J<2 \ell_{1}$. In difference to the previous observations, we examine the case that $\ell_{1}+\ell_{2}>J$. Here we study the monomial

$$
\begin{aligned}
{\left[\mathfrak{R} \beta_{1}\right]^{2} } & \cdots\left[\mathfrak{R} \beta_{J-\ell_{1}}\right]^{2}\left[\mathfrak{I} \beta_{J-\ell_{1}+1}\right]^{3} \\
\cdot & {\left[\mathfrak{R} \beta_{J-\ell_{1}+2}\right]^{3} \cdots\left[\mathfrak{R} \beta_{\ell_{2}}\right]^{3}\left[\mathfrak{R} \beta_{\ell_{2}+1}\right]^{4} \cdots\left[\mathfrak{R} \beta_{J-\ell_{2}}\right]^{4} } \\
\cdot & {\left[\mathfrak{R} \beta_{J-\ell_{2}+1}\right]^{5} \cdots\left[\mathfrak{R} \beta_{\ell_{1}}\right]^{5}\left[\mathfrak{R} \beta_{\ell_{1}+1}\right]^{6} \cdots\left[\mathfrak{R} \beta_{J}\right]^{6}, }
\end{aligned}
$$

where the products over the real part variables with exponent three or four can be empty.

As discussed in (ii), the variables with exponent two cannot arise from quadratic factors [ $\left.\mathfrak{R} \beta_{j}\right]^{2}$ in $\Pi_{\Lambda}$. Consequently, both index sets $\breve{K}_{\ell_{1}}$ and $\breve{K}_{\ell_{2}}$ must contain the indices from 1 to $J-\ell_{1}$. Since only $\ell_{1}$ unassigned variables are left over, these indices must be in $K_{\ell_{1}}$. In other words, $K_{\ell_{1}}$ comprises 
the indices from $J-\ell_{1}+1$ to $J$. Now, the monomial (11.17) is reduced to the unassigned variables in

$$
\begin{aligned}
{\left[\mathfrak{J} \beta_{J-\ell_{1}+1}\right]^{2}\left[\mathfrak{R} \beta_{J-\ell_{1}+2}\right]^{2} \cdots\left[\mathfrak{R} \beta_{\ell_{2}}\right]^{2}\left[\mathfrak{R} \beta_{\ell_{2}+1}\right]^{3} \cdots\left[\mathfrak{R} \beta_{J-\ell_{2}}\right]^{3} } \\
\cdot\left[\mathfrak{R} \beta_{J-\ell_{2}+1}\right]^{4} \cdots\left[\mathfrak{R} \beta_{\ell_{1}}\right]^{4}\left[\mathfrak{R} \beta_{\ell_{1}+1}\right]^{5} \cdots\left[\mathfrak{R} \beta_{J}\right]^{5} .
\end{aligned}
$$

With the same argument as above, the index sets $\breve{K}_{\ell_{1}}$ and $\breve{K}_{\ell_{2}}$ must contain the indices from 1 to $\ell_{2}$. Since $\breve{K}_{\ell_{2}}$ is already completely determined, $\breve{K}_{\ell_{1}}$ must comprise the indices of the variables with exponent three since this variables cannot arise from two quadratic factors of $\Pi_{\Lambda}$. The indices of the remaining $\ell_{2}$ variables now have to be in $K_{\ell_{2}}$, which means that $K_{\ell_{2}}$ must comprise the indices from $J-\ell_{2}+1$ to $J$. At this moment $\breve{K}_{\ell_{1}}$ is still undetermined. However, since the variables with exponent five in the reduced monomial must arise from two quadratic factors in $\Pi_{\Lambda}, \breve{K}_{\ell_{1}}$ can only be extended by the indices of the unassigned variables with exponent four. Therefore, we can finally deduce that the index set $\breve{K}_{\ell_{1}}$ comprises the indices between 1 and $\ell_{1}$.

Summarizing our observations, we can finally conclude that the monomial (11.17) uniquely arises from the factor

$$
\mathfrak{J} \beta_{J-\ell+1} \mathfrak{R} \beta_{J-\ell+2} \cdots \mathfrak{R} \beta_{J}
$$

from $\mathfrak{J}\left[S_{\ell_{1}}(B)\right]$. With a similar argument as in (i), this factor cannot appear in $\mathfrak{R}\left[S_{\ell_{1}}(B)\right]$; so the polynomial in (11.8) cannot vanish everywhere.

(viii) Finally, we come to the last remaining case, where $\ell_{2}<\ell_{1}<J$ with $2 \ell_{1}>$ $2 \ell_{2}>J$. We investigate the monomial

$$
\begin{aligned}
{\left[\mathfrak{R} \beta_{1}\right]^{2} \cdots\left[\mathfrak{R} \beta_{J-\ell_{1}}\right]^{2}\left[\mathfrak{I} \beta_{J-\ell_{1}+1}\right]^{3} } \\
\cdot\left[\mathfrak{R} \beta_{J-\ell_{1}+2}\right]^{3} \cdots\left[\mathfrak{R} \beta_{J-\ell_{2}}\right]^{3}\left[\mathfrak{R} \beta_{J-\ell_{2}+1}\right]^{4} \cdots\left[\mathfrak{R} \beta_{\ell_{2}}\right]^{4} \\
\cdot\left[\mathfrak{R} \beta_{\ell_{2}+1}\right]^{5} \cdots\left[\mathfrak{R} \beta_{\ell_{1}}\right]^{5}\left[\mathfrak{R} \beta_{\ell_{1}+1}\right]^{6} \cdots\left[\mathfrak{R} \beta_{J}\right]^{6} .
\end{aligned}
$$

Analogously to (vii), the index sets $\breve{K}_{\ell_{1}}$ and $\breve{K}_{\ell_{2}}$ contain the indices up to $J-\ell_{1}$, which implies that the remaining $\ell_{1}$ indices from $J-\ell_{1}+1$ to $J$ must be in $K_{\ell_{1}}$. The variables with exponent three hence contain at least one factor being assigned to $K_{\ell_{1}}$. Consequently, these variables cannot arise from any quadratic factors in $\Pi_{\Lambda}$, and the quadratic factors have to cancel out, which means that $\breve{K}_{\ell_{1}}$ and $\breve{K}_{\ell_{2}}$ comprise the indices from 1 to $J-\ell_{2}$. Similarly as above, the remaining $\ell_{2}$ unassigned variables from $J-\ell_{2}+1$ to $J$ must be in $K_{\ell_{2}}$. 
Up to this point, we have completely determined $K_{\ell_{1}}$ and $K_{\ell_{2}}$, and we reduce the original monomial (11.18) to the unassigned product

$$
\left[\mathfrak{R} \beta_{J-\ell_{2}+1}\right]^{2} \cdots\left[\mathfrak{R} \beta_{\ell_{2}}\right]^{2}\left[\mathfrak{R} \beta_{\ell_{2}+1}\right]^{3} \cdots\left[\mathfrak{R} \beta_{\ell_{1}}\right]^{3}\left[\mathfrak{R} \beta_{\ell_{1}+1}\right]^{4} \cdots\left[\mathfrak{R} \beta_{J}\right]^{4} .
$$

In order to determine the last two index sets $\breve{K}_{\ell_{1}}$ and $\breve{K}_{\ell_{2}}$, we proceed as in (iv). In this manner, we finally obtain that both sets $\breve{K}_{\ell_{1}}$ and $\breve{K}_{\ell_{2}}$ comprise the indices from 1 to $\ell_{2}$ because the variables with exponent two in the reduced monomial cannot contain any quadratic factors from $\Pi_{\Lambda}$. Since $\breve{K}_{\ell_{2}}$ is now determined as well, the indices of the variables with exponent three have to be in $\breve{K}_{\ell_{1}}$, which implies that $\breve{K}_{\ell_{1}}$ contains the indices from 1 to $\ell_{1}$.

Thus, the monomial (11.18) uniquely arises from the factor

$$
\mathfrak{J} \beta_{J-\ell+1} \mathfrak{R} \beta_{J-\ell+2} \cdots \mathfrak{R} \beta_{J}
$$

in $\mathfrak{J}\left[S_{\ell_{1}}(B)\right]$. Analogously to the observations in (iv), this factor cannot appear in $\mathfrak{R}\left[S_{\ell_{1}}(B)\right]$, which leads to the conjecture that the polynomial equation (11.8) is non-trivial.

We finally have shown that the polynomial on the left-hand side of (11.8) is non-trivial for all possible combinations of $\ell_{1}, \ell_{2}$, and $J$. Moreover, since the considered monomials have a non-vanishing degree, the polynomial in (11.8) is additionally non-constant, which leads us to the assertion.

Using Lemma 11.6, we can observe that the corresponding zero sets of discretetime signals which fulfil the non-uniqueness conditions in Theorem 11.4 and Corollary 11.5 for a fixed $\ell_{1}$, a fixed $\ell_{2}$, and a fixed subset $\Lambda$ lie on an algebraic variety. This observation implies that almost every discrete-time signal can be uniquely recovered from its FourIER intensity and two additional phases in the time domain.

Theorem 11.7. Let $x$ be a complex-valued discrete-time signal with normalized support of length $N$, and let $\ell_{1}$ and $\ell_{2}$ be different integers between 1 and $N-2$. The phase retrieval problem to recover the signal $x$ from its FOURIER intensity and the two phases $\arg x\left[N-1-\ell_{1}\right]$ and $\arg x\left[N-1-\ell_{2}\right]$ is almost always uniquely solvable whenever $\ell_{1}+\ell_{2} \neq N-1$. In the special case that $\ell_{1}+\ell_{2}=N-1$, the reconstruction is only unique up to conjugate reflections. 
Proof. The assertion follows in an analogous way like Theorem 11.3. Again, we consider the corresponding zero set of a discrete-time signal with normalized support as a $(2 N-2)$-dimensional vector. Further, the corresponding zero sets satisfying the non-uniqueness conditions in Theorem 11.4 and Corollary 11.5 for a specific $\ell_{1}$, a specific $\ell_{2}$, and a specific subset $\Lambda$ lie in the zero locus of a non-constant algebraic polynomial by Lemma 11.2. Due to the fact that there exist only finitely many different subsets $\Lambda$, the exceptional zero sets of signals without a unique reconstruction itself form a set with zero LEBESGUE measure. With VieTA's formulae (Theorem 8.7) and SARD's theorem (Theorem 10.5), the assertion follows as in the proof of Theorem 10.6.

Since the given phases in Theorem 11.7 arise from two inner points, Theorem 11.7 does not cover the observations in Theorem 11.3, where we consider the phase of an inner point and the end point. Anyway, combining the statements of Theorem 11.7 and Theorem 11.3, and remembering that the additional phase information of the two end points cannot reduce the set of non-trivial ambiguities, we finally show that almost all signals can be recovered from their FourIER intensities and two arbitrarily given phases in the time domain except for two special cases.

Corollary 11.8. Let $x$ be a complex-valued discrete-time signal with normalized support of length $N$, and let $\ell_{1}$ and $\ell_{2}$ be different integers between 0 and $N-1$. The phase retrieval problem to recover the signal $x$ from its FOURIER intensity and the two phases $\arg x\left[N-1-\ell_{1}\right]$ and $\arg x\left[N-1-\ell_{2}\right]$ is almost always uniquely solvable whenever $\ell_{1}+\ell_{2} \neq N-1$. If $\ell_{1}+\ell_{2}=N-1$, then the reconstruction is only unique up to conjugate reflections, except for the special case where $\ell_{1}$ and $\ell_{2}$ correspond to the two end points.

\subsection{The phase of the entire signal}

In analogy to our considerations in Section 10.3, we light upon the issue whether each discrete-time signal can be recovered without any ambiguities - trivial or non-trivial - if only the phase information of enough points in the time domain is given. Looking back at Corollary 11.8, we can immediately justify that at least almost every signal can be absolutely uniquely recovered from its FouRIER intensity and the complete phase information $\arg x:=(\arg x[n])_{n \in \mathbb{Z}}$ in the time domain. 
Corollary 11.9. Let $x$ be a complex-valued discrete-time signal with normalized support of length $N$. The phase retrieval problem to recover the signal $x$ from its FOURIER intensity $|\widehat{x}|$ and its phases $\arg x$ in the time domain is almost always uniquely solvable.

Unfortunately, the complete phase information of a signal fails to enforce the absolute uniqueness of the phase retrieval problem for every discrete-time signal. Interpreting the recovery of a real-valued non-negative signal from its FOURIER intensity as the phase retrieval problem to reconstruct a complex-valued signal whose phases are constantly zero in the time domain, and remembering our investigation of the non-trivial ambiguities in phase retrieval of non-negative signals (Chapter II), we can easily give appropriate counter-examples.

Theorem 11.10. For every integer $N>2$, there exists a discrete-time signal $x$ with normalized support of length $N$ such that the phase retrieval problem to recover the signal $x$ from its FOURIER intensity $|\widehat{x}|$ and its phases $\arg x$ possesses at least a further non-trivial ambiguity.

Proof. With the intent to construct a suitable counter-example, we consider a discrete-time signal $x$ whose corresponding zeros lie on the real axis in the left half plane. Choosing the corresponding zeros in this manner, we ensure that the considered signal $x$ and all arising ambiguities are real-valued. Moreover, Proposition 9.1 yields that the considered signal itself and all non-trivial ambiguities are non-negative. In other words, the phase of all non-trivial solutions is constantly zero. Hence, if the corresponding zero set contains at least two zeros not lying on the unit circle, we find a further non-trivial solution with phase zero in the time domain.

Remark 11.11. In order to prove Proposition 9.13, we already constructed nonnegative signals for every support length greater then three. Nevertheless, considering the proof of Theorem 11.10, we can immediately observe that at worst the additional phase information cannot reduce the set of non-trivial ambiguities at all. For instance, this happens if the corresponding zeros are pairwise distinct, do not occur in reflected pairs, and do not lie on the unit circle, see Proposition 6.1. 


\section{Using additionally given points of the signal}

Our considerations of the phase retrieval problem with additionally given moduli or phases of the unknown signal were inspired by the work of Xu et al., who investigate the recovery of a real-valued signal $x$ from its FourIER intensity $|\widehat{x}|$ and its end point $x[N-1]$, see [XYC87]. In order to return to this starting point, we will now transfer this approach and briefly consider the phase retrieval problem to recover an unknown complex-valued signal from its Fourier intensity and one additionally given signal value in the time domain.

The trivial rotation ambiguity allows us to adjust the phase of one signal value in the time domain. Thus, this specific phase retrieval problem is uniquely solvable if we can recover the unknown signal from its FourIER intensity and the absolute value of the given point up to rotations and vice versa. In other words, if we consider only one additional signal value in the time domain, then we obtain the same results as in Section 10, where the additionally known phase eliminates the trivial rotation ambiguity. In this manner, Theorem 10.11 immediately entails the following observation.

Corollary 12.1. Let $x$ be a complex-valued discrete-time signal with normalized support of length $N$, and let $\ell$ be an arbitrary integer between 0 and $N-1$. The phase retrieval problem to recover the signal $x$ from its FOURIER intensity $|\widehat{x}|$ and the signal value $x[N-1-\ell]$ is almost always uniquely solvable whenever $\ell \neq(N-1) / 2$. In the special case that $\ell=(N-1) / 2$, the reconstruction is only unique up to conjugate reflections.

As a consequence of Corollary 12.1, we can also recover almost every signal uniquely if we know more then one signal value in the time domain exactly. Moreover, if the complete signal $x$ itself is given, then the solution of the phase retrieval problem to recover the 'unknown' signal is obviously unique. Hence, the question arises: how many signal values are at least needed to solve the phase retrieval problem always uniquely?

In [NQL83b, Theorem 2], NAwAB, QUATIERI, and Lim show that the phase retrieval problem to recover a real-valued signal $x$ with normalized support of length $N$ is uniquely solvable if at least the $\lceil N / 2\rceil$ right end points

$$
x[\lfloor N / 2\rfloor], \ldots, x[N-1]
$$

are additionally given. Here $\lfloor N / 2\rfloor$ denotes the largest integer less than or equal to $N / 2$, and $\lceil N / 2\rceil$ the smallest integer greater than or equal to $N / 2$. Following the 
original proof of NAWAB et al. for the real phase retrieval problem, we can easily generalize this observation for complex-valued signals.

Proposition 12.2. Let $x$ be a complex-valued discrete-time signal with normalized support of length $N$. Then the signal $x$ can be uniquely recovered from its FOURIER intensity $|\widehat{x}|$ and the $\lceil N / 2\rceil$ right end points

$$
x[\lfloor N / 2]], \ldots, x[N-1] .
$$

Proof. Looking back at Proposition 3.4, where we have shown that the squared FourIER intensity $|\widehat{x}|^{2}$ are equal to the autocorrelation function $\widehat{a}$, we can determine the autocorrelation signal $a$ of the unknown signal $x$ from the given data by using the inverse discrete-time FoURIER transform. Since the components of the autocorrelation signal (Definition 3.1) are defined by

$$
a[n]:=\sum_{k \in \mathbb{Z}} \overline{x[k]} x[k+n],
$$

the components of the unknown signal $x$ with normalized support usually have to satisfy a quadratic equation system.

However, with the a priori known right end points $x[\lfloor N / 2\rfloor], \ldots, x[N-1]$, the remaining components can be recovered by solving the linear equation system

$$
\left(\begin{array}{cccc}
\overline{\frac{x[N-1]}{x[N-2]}} & \overline{x[N-1]} & & \\
\frac{\vdots}{x\left[\left\lceil\frac{N}{2}\right\rceil\right]} & \frac{\vdots}{x\left[\left\lceil\frac{N}{2}\right\rceil+1\right]} & \ldots & \overline{x[N-1]}
\end{array}\right)\left(\begin{array}{c}
x[0] \\
x[1] \\
\vdots \\
x\left[\left\lfloor\frac{N}{2}\right\rfloor-1\right]
\end{array}\right)=\left(\begin{array}{c}
\overline{a[N-1]} \\
\overline{a[N-2]} \\
\vdots \\
\hline a\left[\left\lceil\frac{N}{2}\right]\right]
\end{array}\right)
$$

analogously to the real case in [NQL83b, p. 988]. Since the quadratic matrix is a lower left triangle matrix, and $x[N-1]$ is non-zero because $x$ has a normalized support of length $N$, this linear equation system has a unique solution.

Remark 12.3. In the original proof of the real counterpart of Proposition 12.2, NAWAB et al. furthermore show that the phase retrieval problem with additionally given end points is uniquely solvable if and only if at least $\lceil N / 2\rceil$ right end points are given. Unfortunately, the counter-examples in [NQL83b] are merely rotations and reflections of the original signal. As a consequence, we cannot exclude that the phase retrieval problem is uniquely solvable up to trivial ambiguities from less than $\lceil\mathrm{N} / 2\rceil$ end points. 



\title{
Enforcing uniqueness by interference measurements
}

\begin{abstract}
We will now consider a class of discrete-time phase retrieval problems where more than one intensity measurement in the frequency domain is given. Firstly, we will assume that the additional FourIER intensity arises from the interference with a known or an unknown reference signal. For the special case that the reference is a known DiRAC signal, we will straightforwardly transfer the observations of KIM and HAYEs [KH9ob] for real-valued signals to the complex setting. Generalizing our findings to arbitrary known reference signals, we will here obtain an explicit representation of all further solutions. If the reference signal is also unknown, KIm and Hayes [KH93] have shown that most real-valued signals are determined by three FourIER intensities. For complex-valued signals, RAZ, DUDOvich, and NADLER [RDN13] have obtained a similar result by employing a fourth intensity. Using our characterization, we will here give a new and complete proof for complex-valued signals and only three FourIER intensities as in the real setting. Finally, we will investigate interference measurements of the unknown signal with a modulation of the signal itself. Using PronY's method, we will here adapt and extend the results in [ABFM14] and [CESV13] to show that each discrete-time signal with finite support can always be recovered uniquely. $\triangleleft$
\end{abstract}

${ }^{a}$ The observations in this chapter have been partly published in [BP15a].

\section{Interference with a known reference signal}

Up to this point, the a priori restrictions and additional information used to reduce the number of non-trivial ambiguities in the discrete-time phase retrieval problem are located in the time domain. Nevertheless, in some applications like FOURIER holography, for instance, further intensity measurements besides the FOURIER intensity of the unknown signal are available in the frequency domain. More precisely, this additional FouRIER intensity results from the interference of the original signal with a known or unknown reference signal. 
Using interference measurements with a suitable known reference signal, KIM and HAYES prove that a real-valued discrete-time signal can be always recovered except for possibly one trivial ambiguity, see [KH9ob]. We generalize the approach of KIM and HAYES and exploit the additional interference measurements to enforce uniqueness of the phase retrieval problem for complex-valued signals.

\subsection{Interference with a known Dirac signal}

Firstly, we restrict ourselves to interference measurements with a known DiRAC signal $C \delta_{n_{0}}$, where $C$ is a non-zero complex number, and $n_{0}$ an arbitrary integer. In other words, we try to recover the complex-valued signal $x$ with finite support from its FourIER intensity $|\mathcal{F}[x]|=|\widehat{x}|$ and the FourIER intensity $\left|\mathcal{F}\left[x+C \delta_{n_{0}}\right]\right|$ of the interference of $x$ with $C \delta_{n_{0}}$. Generalizing the observations of KIM and HAYES in [KH9ob, Theorem 1] to the complex case, we can uniquely recover the original signal except for one specific trivial ambiguity.

Theorem 13.1. Let $x$ be a discrete-time signal with finite support. Furthermore, let $C$ and $n_{0}$ be an a priori known non-zero complex number and an a priori known integer respectively. Then the signal $x$ can be recovered from the FOURIER intensities

$$
|\mathcal{F}[x]| \text { and }\left|\mathcal{F}\left[x+C \delta_{n_{0}}\right]\right|
$$

except for at most one trivial ambiguity.

Proof. In order to establish this theorem, we adapt the original proof given by KIM and HAYEs in [KH9ob, p. 442 et seq.] to reconstruct a real-valued signal. For the sake of simplicity, we let $y$ denote the interference of $x$ with the known DiRAC signal $C \delta_{n_{0}}$. Then, the FouRIER transform of the interference $y:=x+C \delta_{n_{0}}$ is given by

$$
\widehat{y}(\omega)=\widehat{x}(\omega)+C \mathrm{e}^{-\mathrm{i} \omega n_{0}} .
$$

Further, with the polar representations $\widehat{x}(\omega)=|\widehat{x}(\omega)| \mathrm{e}^{\mathrm{i} \phi(\omega)}$ for $\omega \in \mathbb{R}$ and $C=|C| \mathrm{e}^{\mathrm{i} \psi}$, where $\phi(\omega)$ and $\psi$ denote the phases of $\hat{x}$ and $C$, we can write the squared FOURIER intensity of the interference $y$ as

$$
|\widehat{y}(\omega)|^{2}=|\widehat{x}(\omega)|^{2}+2|C||\widehat{x}(\omega)| \cos \left(\phi(\omega)+n_{0} \omega-\psi\right)+|C|^{2}
$$

by using the law of cosines. Within this equation, the unknown phase $\phi$ of the FOURIER transformed signal $x$ is directly encoded. Rearranging the terms of this 
equality, we obtain the pointwise identity

$$
\cos \left(\phi(\omega)+n_{0} \omega-\psi\right)=\frac{|\widehat{y}(\omega)|^{2}-|\widehat{x}(\omega)|^{2}-|C|^{2}}{2|C||\widehat{x}(\omega)|}
$$

whenever $|\widehat{x}(\omega)|$ is non-zero.

Moreover, since $\widehat{x}$ is a non-vanishing trigonometric polynomial and hence has only finite many zeros, this identity holds almost everywhere. Consequently, we can determine the unknown phase $\phi$ for almost every point $\omega$ in the frequency domain by using the arc cosine. More detailed, the phase at a certain point $\phi(\omega)$ is given by the equation

$$
\phi(\omega)+n_{0} \omega-\psi= \pm \arccos \left(\frac{|\widehat{y}(\omega)|^{2}-|\widehat{x}(\omega)|^{2}-|C|^{2}}{2|C||\widehat{x}(\omega)|}\right)+2 \pi k,
$$

where $k$ is a suitable integer.

Using again that $\widehat{x}$ is a trigonometric polynomial, we can conclude that the unknown phase $\phi$ can be written as a piecewise continuous function on its domain. Hence, the sign of the arc cosine and the integer $k$ in (13.1) cannot be chosen (completely) arbitrarily, and we find at least a small open interval where the sign is everywhere plus or everywhere minus and $k$ does not change. Knowing the phase $\phi$ and hence the trigonometric polynomial $\widehat{x}$ on an open interval, we can uniquely extend the phase $\phi$ and the trigonometric polynomial $\widehat{x}$ to the complete frequency domain. As an immediate consequence, the considered phase retrieval problem can have at most two distinct solutions.

Now, let $\breve{x}$ be the second possible solution of the considered phase retrieval problem. This means that the discrete-time signal $\breve{x}$ has to fulfil the equations

$$
|\mathscr{F}[x]|=|\mathscr{F}[\breve{x}]| \text { and }\left|\mathscr{F}\left[x+C \delta_{n_{0}}\right]\right|=\left|\mathscr{F}\left[\breve{x}+C \delta_{n_{0}}\right]\right| \text {. }
$$

Analogously to the original signal $x$, let $\breve{\phi}$ denote the phase of $\mathscr{F}[\breve{x}]$. As discussed above, we assume that the sign of the arc cosine in (13.1) for the original phase $\phi(\omega)$ and the second phase $\breve{\phi}(\omega)$ differ on an open interval. By equation (13.1), a comparison of the obtained representations for $\phi(\omega)$ and $\breve{\phi}(\omega)$ yields the identity

$$
\phi(\omega)+n_{0} \omega-\psi=-\left(\breve{\phi}(\omega)+n_{0} \omega-\psi\right)+2 \pi k
$$

or

$$
\breve{\phi}(\omega)=-\phi(\omega)-2 n_{0} \omega+2 \psi+2 \pi k
$$

for almost every $\omega$ in the frequency domain, where $k$ is again an appropriately chosen integer. 
Bearing in mind that differences in the phase by a multiple of $2 \pi$ do not change a complex-valued function at all, we can thus deduce that the FOURIER transform of the second possible solution $\breve{x}$ is given by

$$
\widehat{\breve{x}}(\omega)=|\widehat{x}(\omega)| \mathrm{e}^{-\mathrm{i} \phi(\omega)-2 \mathrm{in} n_{0} \omega+2 \mathrm{i} \psi} .
$$

As seen in the proof of Proposition 2.1 and in Example 2.2, the conjugation, modulation, and rotation in the frequency domain cause trivial ambiguities in the time domain. More precisely, the inverse discrete-time FouRIER transform yields the signal

$$
\breve{x}=\mathrm{e}^{2 \mathrm{i} \psi} \overline{x\left[2 n_{0}-\cdot\right]},
$$

which is obviously a trivial ambiguity of $x$ caused by rotation, time shift, and reflection and conjugation. Hence, the considered phase retrieval problem can have at most one further trivial solution as conjectured in the assertion.

Remark 13.2. Looking back at Remark 3.5, we already know that the FoURIER transform of a discrete-time signal with support length $N$ is completely determined by $2 N-1$ discrete measurements in $[-\pi, \pi)$. Consequently, if $n_{0}$ is contained in the support of the original signal, then we only need $4 N-2$ measurements, $2 N-1$ for each FourIER intensity, in order to recover the unknown signal. If $n_{0}$ is not contained in the support, then the support of the interference signal $y=x+C \delta_{n_{0}}$ in Theorem 13.1 is enlarged, which results in a higher number of required measurements for the corresponding FOURIER intensity.

Remark 13.3. Based on an approach of KIM and HAYEs for real-valued signals, we can sometimes solve the phase retrieval problem in Theorem 13.1 without knowing the FourIER intensity of $x$ itself. Following and adapting the ideas in [KH9ob, p. 443] to the complex case, we assume that the unknown signal $x$ has a normalized support of length $N$, and that $n_{0}$ is greater than $2 N-2$ or less than $-N+1$.

As in the proof of Theorem 13.1, let $y$ denote the interference of the original signal $x$ with a known Dirac signal $C \delta_{n_{0}}$ such that

$$
\widehat{y}(\omega)=\widehat{x}(\omega)+C \mathrm{e}^{\mathrm{i} \omega n_{0}} .
$$

Instead of applying the law of cosines, we simply extend the squared Fourier intensity and obtain

$$
|\widehat{y}(\omega)|^{2}=|\widehat{x}(\omega)|^{2}+\bar{C} \mathrm{e}^{-\mathrm{i} \omega n_{0}} \widehat{x}(\omega)+C \mathrm{e}^{\mathrm{i} \omega n_{0}} \overline{\widehat{x}(\omega)}+|C|^{2}
$$


for every $\omega$ in the frequency domain. Since $n_{0}$ is less than $-N+1$ or greater than $2 N-2$, the coefficients of the three trigonometric polynomials

$$
|\widehat{x}(\omega)|^{2}+|C|^{2}, \quad \bar{C} \mathrm{e}^{-\mathrm{i} \omega n_{0}} \widehat{x}(\omega) \quad \text { and } \quad C \mathrm{e}^{\mathrm{i} \omega n_{0}} \overline{\widehat{x}(\omega)}
$$

do not superpose. The Fourier transform $\widehat{x}$ of the unknown signal $x$ is thus completely encoded in $|\widehat{y}|^{2}$ and can be simply determined by considering the corresponding coefficients of the autocorrelation function of $y$.

Shifting both the original signal $x$ and the known reference signal $C \delta_{n_{0}}$, this observation can be easily generalized to discrete-time signals with an arbitrary finite support.

\subsection{Interference with a known reference signal}

Let us now consider interference measurements with a known but arbitrary reference signal. In other words, we replace the DIRAC signal in Section 13.1 by an arbitrary discrete-time signal with finite support. KIM and HAYES also examine real phase retrieval problems of this kind in their work [KH9ob], but with the restriction that all occurring signals are real-valued and non-symmetric. We use a slightly different approach and generalize our findings in Section 13.1.

Theorem 13.4. Let $x$ and $h$ be two discrete-time signals with finite support, where the non-vanishing reference signal $h$ is known beforehand. Then the signal $x$ can be recovered from the FOURIER intensities

$$
|\mathscr{F}[x]| \text { and }|\mathcal{F}[x+h]|
$$

except for at most one ambiguity.

Proof. The assertion can simply be obtained by generalizing the procedure in the proof of Theorem 13.1. We denote by $y$ the interference of the original signal $x$ with the reference signal $h$. Since $x$ and $h$ are discrete-time signals with finite support, the support of the interference $y$ is finite too. Further, the Fourier transform of $y$ is given by

$$
\widehat{y}(\omega)=\widehat{x}(\omega)+\widehat{h}(\omega)
$$

With the intent to investigate the squared FourIER intensity $|\widehat{y}|^{2}$, we write the FOURIER transforms of the unknown signal $x$ and the known reference signal $h$ in their polar representation $\widehat{x}(\omega)=|\widehat{x}(\omega)| \mathrm{e}^{\mathrm{i} \phi(\omega)}$ and $\widehat{h}(\omega)=|\widehat{h}(\omega)| \mathrm{e}^{\mathrm{i} \psi(\omega)}$ 
respectively, where $\phi(\omega)$ and $\psi(\omega)$ denote the phase functions of $\widehat{x}(\omega)$ and $\widehat{h}(\omega)$. Analogously to the proof of Theorem 13.1, the law of cosines now yields the identity

$$
|\widehat{y}(\omega)|^{2}=|\widehat{x}(\omega)|^{2}+2|\widehat{x}(\omega)||\widehat{h}(\omega)| \cos (\phi(\omega)-\psi(\omega))+|\widehat{h}(\omega)|^{2},
$$

which results in the pointwise characterization of the unknown phase $\phi$ by

$$
\phi(\omega)-\psi(\omega)= \pm \arccos \left(\frac{|\widehat{y}(\omega)|^{2}-|\widehat{x}(\omega)|^{2}-|\widehat{h}(\omega)|^{2}}{2|\widehat{x}(\omega)||\widehat{h}(\omega)|}\right)+2 \pi k
$$

with an appropriate integer $k$ whenever $\widehat{x}(\omega)$ and $\widehat{h}(\omega)$ are non-zero.

Because $\widehat{x}$ and $\widehat{h}$ are trigonometric polynomials, we can use a similar argumentation as in the proof of Theorem 13.1 in order to conclude that the considered phase retrieval problem can have at most two different solutions. Again, let $\breve{x}$ denotes the second possible solution, and $\breve{\phi}(\omega)$ the corresponding phase in the frequency domain.

Following the lines of the original proof, we assume that the sign of the arc cosine in (13.2) for the two phase functions $\phi(\omega)$ and $\breve{\phi}(\omega)$ differ. Then, the corresponding phases of both solutions are related by

$$
\breve{\phi}(\omega)=-\phi(\omega)+2 \psi(\omega)+2 \pi k
$$

for almost every $\omega$ in the frequency domain, where $k$ again is a suitable integer depending on $\omega$. Therefore, the Fourier transform of the second solution $\breve{x}$ is given by

$$
\widehat{\breve{x}}(\omega)=|\widehat{x}(\omega)| \mathrm{e}^{-\mathrm{i} \phi(\omega)+2 \mathrm{i} \psi(\omega)} .
$$

Depending on the phase $\psi(\omega)$ of the reference signal $h$ in the frequency domain, the discrete-time signal $\breve{x}$ can be a trivial or non-trivial ambiguity, which completes the proof.

The behaviour of the occurring ambiguity in Theorem 13.4 mainly depends on the phase $\psi$ of the known reference signal in the frequency domain. In order to decide whether the possible second solution determined by

$$
\widehat{\breve{x}}(\omega)=|\widehat{x}(\omega)| \mathrm{e}^{-\mathrm{i} \phi(\omega)+2 \mathrm{i} \psi(\omega)}
$$

is trivial or non-trivial, we investigate some special cases. 
Firstly, we assume that the reference signal $h$ possesses a linear phase. This means that the Fourier transform of $h$ can be written in the form

$$
\widehat{h}(\omega)=|\widehat{h}(\omega)| \mathrm{e}^{-\mathrm{i} \omega n_{0}+\mathrm{i} \alpha}
$$

with an integer $n_{0}$ and a real number $\alpha$, see for instance [OS89, Section 5.7.1]. As their real-valued counterpart in [OS89], complex-valued signals with linear phase can be characterized by a simple symmetry property in the time domain.

Proposition 13.5. Let $h$ be a discrete-time signal with finite support. The signal $h$ possesses a linear phase if and only if the components of $h$ fulfil

$$
h[n]=\mathrm{e}^{2 \mathrm{i} \alpha} h\left[2 n_{0}-n\right]
$$

for every integer $n$ for an appropriate integer $n_{0}$ and real number $\alpha$.

Proof. We start with a discrete-time signal $h$ with finite support and linear phase. Hence, there exist an integer $n_{0}$ and a real number $\alpha$ such that $h$ can be written as

$$
\widehat{h}(\omega)=|\widehat{h}(\omega)| \mathrm{e}^{-\mathrm{i} \omega n_{0}+\mathrm{i} \alpha}
$$

in the frequency domain. Bringing the linear phase factor $\mathrm{e}^{-\mathrm{i} \omega n_{0}+\mathrm{i} \alpha}$ to the other side and considering that the amplitude $|\widehat{h}|$ is real-valued, we obtain the identity

$$
\mathrm{e}^{\mathrm{i} \omega n_{0}-\mathrm{i} \alpha} \widehat{h}(\omega)=|\widehat{h}(\omega)|=\overline{|\widehat{h}(\omega)|}=\mathrm{e}^{-\mathrm{i} \omega n_{0}+\mathrm{i} \alpha} \overline{\widehat{h}(\omega)} .
$$

Thus, the inverse discrete-time FourIER transform implies that the components of the signal $h$ have to satisfy the symmetry

$$
\mathrm{e}^{-\mathrm{i} \alpha} h\left[n_{0}+n\right]=\mathrm{e}^{\mathrm{i} \alpha} h\left[n_{0}-n\right] .
$$

A rearrangement of this symmetry establishes the proposition.

Considering the ambiguity in Theorem 13.4, we can immediately conclude that the ambiguity in the phase retrieval problem for a known reference signal with linear phase have to be trivial. 
Corollary 13.6. Let $x$ and $h$ be two discrete-time signals with finite support, where the non-vanishing reference signal $h$ is known beforehand. If the signal $h$ furthermore possesses a linear phase, then the signal $x$ can be recovered from the FOURIER intensities

$$
|\mathcal{F}[x]| \text { and }|\mathcal{F}[x+h]|
$$

except for one trivial ambiguity at the most.

Proof. Since the phase $\psi$ of the reference signal $h$ is linear phase, it can be written as

$$
\psi(\omega)=-n_{0} \omega+\alpha
$$

where $n_{0}$ is an integer and $\alpha$ a real number. Hence, the second solution $\breve{x}$ in the proof of Theorem 13.4 is determined by

$$
\widehat{\breve{x}}(\omega)=|\widehat{x}(\omega)| \mathrm{e}^{-\mathrm{i} \phi(\omega)-2 \mathrm{i} \omega n_{0}+2 \mathrm{i} \alpha} .
$$

Therefore, $\breve{x}$ arises from a rotation, time shift, and reflection and conjugation of $x$ similarly to the proof of Theorem 13.1, which leads us to the assertion.

Remark 13.7. In the real version of the considered phase retrieval problem in Corollary 13.6, where all occurring signals are real-valued, an analogous behaviour can be observed as briefly mentioned by KIM and HaYes in [KH9ob, p. 443]. Assuming that the signal $h$ in Proposition 13.5 possesses only real components, the reference signals with linear phase can be divided into two different types, namely signals with an even symmetry $h[n]=h\left[2 n_{0}-n\right]$ and the signals with an odd symmetry $h[n]=-h\left[2 n_{0}-n\right]$ for a certain integer $n_{0}$.

Remark 13.8. Theoretically, the ambiguity in Theorem 13.4 is also trivial if we assume that the reference signal $h$ has a phase of the form

$$
\psi(\omega)=-\frac{n_{0}}{2} \omega+\frac{\alpha}{2}
$$

in the frequency domain, where $n_{0}$ is again an integer, and $\alpha$ a real number. Although the signal $h$ possesses a 'linear phase', the Fourier transform $\mathscr{F}[h]$ is not a $2 \pi$-periodic function. In other words, such a discrete-time signal $h$ does not exist.

Besides the trivial ambiguity caused by a reference signal with linear phase, the phase retrieval problem in Theorem 13.4 can also have a non-trivial ambi- 
guity. Since the second solution $\breve{x}$ in the proof of Theorem 13.4 is determined by

$$
\widehat{\breve{x}}=|\widehat{x}| \mathrm{e}^{-\mathrm{i} \phi+2 \mathrm{i} \psi},
$$

where $\phi$ and $\psi$ are the phase functions of the original signal $x$ and the reference signal $h$ in the frequency domain, we can only obtain a further non-trivial ambiguity if the reference signal contains the phase of this ambiguity.

Corollary 13.9. Let $x$ and $h$ be two discrete-time signals with finite support. Assume that the reference signal $h$ is known beforehand and possesses a phase of the form

$$
\arg \widehat{h}(\omega)=-n_{0} \omega+\alpha+\arg \left(\prod_{\beta_{j} \in B \backslash \Lambda}\left(\mathrm{e}^{-\mathrm{i} \omega}-\beta_{j}\right)\right)
$$

where $\Lambda$ is a non-empty, proper subset of the corresponding zero set $B$ of the signal $x$. If $\Lambda$ and $B \backslash \Lambda$ are not invariant under reflection at the unit circle, then the phase retrieval problem to recover $x$ from the FOURIER intensities

$$
|\mathcal{F}[x]| \text { and }|\mathscr{F}[x+h]|
$$

has exactly one further solution beside $x$, and this solution is non-trivial.

Proof. To simplify the notation in the following, we assume without loss of generality that the original signal $x$ has a normalized support of length $N$, and that the leading component $x[N-1]$ is a positive real number. In this manner, we avoid the additional rotation and shift within the characterization in Theorem 5.1. This implies that the phase $\phi$ of the signal $x$ in the frequency domain is given by

$$
\arg \widehat{x}(\omega)=\arg \left(\prod_{j=1}^{N-1}\left(\mathrm{e}^{-\mathrm{i} \omega}-\beta_{j}\right)\right)
$$

Combining the representations of the phase functions $\phi:=\arg \widehat{x}$ and $\psi:=$ $\widehat{h}$, the phase $-\phi+2 \psi$ corresponding to the second solution $\breve{x}$ in the proof of Theorem 13.4 can thus be written as

$$
\arg \widehat{\breve{x}}(\omega)=-2 n_{0} \omega+2 \alpha+\arg \left(\overline{\prod_{\beta_{j} \in \Lambda}\left(\mathrm{e}^{-\mathrm{i} \omega}-\beta_{j}\right)} \cdot \prod_{\beta_{j} \in B \backslash \Lambda}\left(\mathrm{e}^{-\mathrm{i} \omega}-\beta_{j}\right)\right) .
$$

In other words, except for an additional rotation and modulation, we replace the phase of the original signal $x$ in the frequency domain by the phase of the signal 
$\breve{x}$ where we reflect and conjugate the linear factors $\delta_{1}-\beta_{j} \delta_{0}$ for the zeros $\beta_{j}$ in the subset $\Lambda$.

Consequently, the second solution $\breve{x}$ corresponds to the modified zero set $B^{(\Lambda)}$, which can be seen by using Lemma 5.3 for instance. Moreover, the modified zero set $B^{(\Lambda)}$ and the original corresponding zero set $B$ cannot coincide since $\Lambda$ is not invariant under reflection at the unit circle. A similar observation holds true for the modified zero set $B^{(\Lambda)}$ and the reflection of $B$ since $B \backslash \Lambda$ also is not invariant under the reflection. In other words, the modified zero set $B^{(\Lambda)}$ cannot coincide with the corresponding zeros of the original signal $x$ or the reflected and conjugated signal $\overline{x[-\cdot]}$, which implies that the two solutions $x$ and $\breve{x}$ are non-trivially different as claimed.

Example 13.10. Considering the phase function of the reference signal $h$ in the frequency domain in Corollary 13.9, the non-trivial ambiguity especially appears if the reference signal $h$ contains linear factors of the unknown signal. In order to give an explicit instance, we revisit the phase retrieval problem in Example 1.1. This means that we try to recover the signal

$$
\begin{aligned}
x:=\frac{1}{128}(\ldots, 0, \underline{55-15 i},-84+87 \mathrm{i}, 34+82 \mathrm{i}, \\
204-120 \mathrm{i},-16+16 \mathrm{i},-96,128,0, \ldots) .
\end{aligned}
$$

The original signal and the corresponding FouRIER intensity are graphically shown in Figure 13.1 on the next page.

In this specific example, the original signal $x$ corresponds to the zero set

$$
B=\frac{1}{4}\{1+\mathrm{i}, 3-2 \mathrm{i},-3-\mathrm{i},-4+2 \mathrm{i}, 4+4 \mathrm{i}, 2-4 \mathrm{i}\} .
$$

With the intent to construct a reference signal $h$ such that the ambiguity in Theorem 13.4 becomes non-trivial, we choose the last four zeros in $B$ for the corresponding zero set of $h$. In other words, we define the subset $\Lambda$ by

$$
\Lambda:=\frac{1}{4}\{1+\mathrm{i}, 3-2 \mathrm{i}\}
$$

and the reference signal $h$ by

$$
h:=\delta_{1} * \underset{\beta_{j} \in B \backslash \Lambda}{\mathbb{*}}\left(\delta_{1}-\beta_{j} \delta_{0}\right) .
$$

Consequently, the signal $h$ has the form

$$
h=\frac{1}{32}(\ldots, 0, \underline{0}, 20-10 \mathrm{i}, 19-17 \mathrm{i},-4-4 \mathrm{i}, 4-4 \mathrm{i}, 16,0, \ldots) .
$$




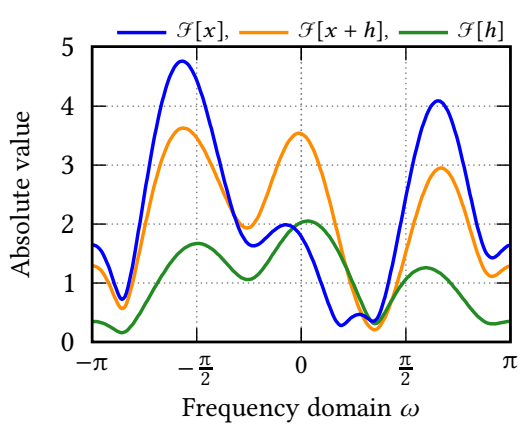

(a) FourIER intensities $\mathscr{F}[x]$ and $\mathscr{F}[h]$, and the interference measurements $\mathscr{F}[x+h]$ with the known reference $h$

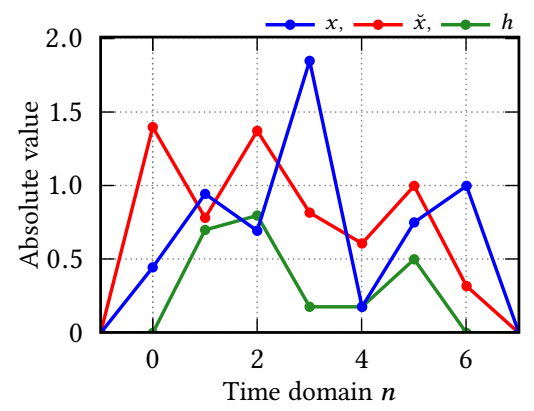

(c) Absolute values of the signals $x$ and $\breve{x}$ and of the reference signal $h$ illustrated by polygonal lines

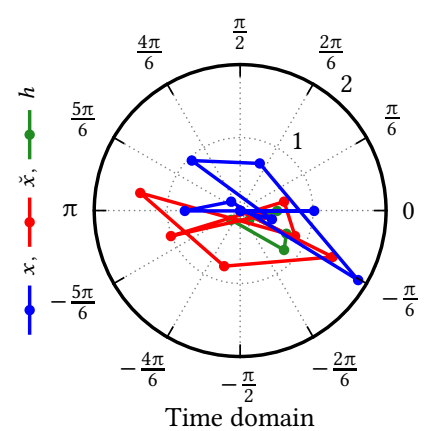

(b) Signals $x$ and $\breve{x}$ together with the reference signal $h$ represented in polar form

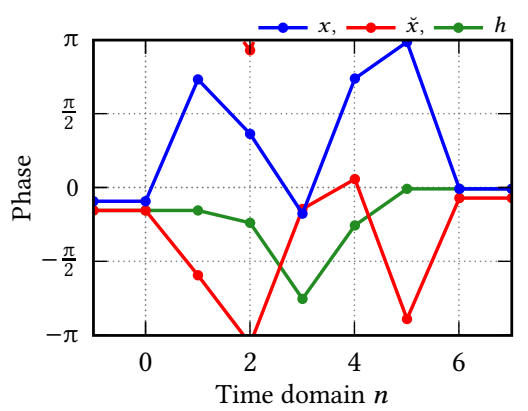

(d) Phases of the signals $x$ and $\breve{x}$ and of the reference signal $h$ illustrated by polygonal lines

Figure 13.1.: Discrete-time phase retrieval problem to recover the signal $x$ from the FourIER intensities $\mathscr{F}[x]$ and $\mathscr{F}[x+h]$ for a known reference signal $h$ with a second non-trivial solution

Obviously, the phase of the reference signal $h$ in the frequency domain fulfils the assumptions of Corollary 13.9 by construction; so the phase retrieval problem to recover $x$ from the FourIER intensities $|\mathcal{F}[x]|$ and $|\mathcal{F}[x+h]|$ shown in Figure 13.1(a) possesses exactly one further non-trivial solution $\breve{x}$. More precisely, following the proof of Corollary 13.9, the second solution $\breve{x}$ corresponds to the modified zero set $B^{(\Lambda)}$, which results in the representation

$$
\begin{aligned}
\breve{x}=\frac{1}{128}(\ldots, 0, \underline{160-80 \mathrm{i}},-28-96 \mathrm{i},-173+31 \mathrm{i}, \\
95-44 \mathrm{i}, 76+16 \mathrm{i},-120-44 \mathrm{i}, 40-8 \mathrm{i}, 0, \ldots) .
\end{aligned}
$$


Considering Figure 13.1(c) and (d), we can also validate the non-trivial ambiguousness between $x$ and $\breve{x}$ visually.

Remark 13.11. If the subset $\Lambda$ or $B \backslash \Lambda$ in Corollary 13.9 is invariant under reflection at the unit circle, then the constructed second solution in the corresponding proof becomes trivial. Summarizing our observations in Corollary 13.6 and Corollary 13.9, we can hence conclude that the phase retrieval problem in Theorem 13.4 possesses a further solution - trivial or non-trivial - if the reference signal $h$ has a phase of the form

$$
\arg \widehat{h}(\omega)=-n_{0} \omega+\alpha+\arg \left(\prod_{\beta_{j} \in B \backslash \Lambda}\left(\mathrm{e}^{-\mathrm{i} \omega}-\beta_{j}\right)\right),
$$

where $\Lambda$ is an arbitrary subset of the corresponding zero set $B$ of the signal $x$.

Conversely, if the reference signal $h$ does not possess a phase of this form, then the second solution $\breve{x}$ determined by

$$
\widehat{\breve{x}}=|\widehat{x}| \mathrm{e}^{-\mathrm{i} \arg \widehat{x}+2 \mathrm{i} \arg \widehat{h}}=|\widehat{x}| \mathrm{e}^{-\mathrm{i} \phi+2 \mathrm{i} \psi}
$$

in the proof of Theorem 13.4 cannot coincide with a solution of the discretetime phase retrieval problem characterized in Theorem 5.1. In other words, the constructed signal $\breve{x}$ cannot have a finite support and is hence invalid. As a consequence, by choosing a known reference signal without a linear phase, the phase retrieval problem with additional interference measurements is usually uniquely solvable for most discrete-time signals $x$ with the exception of rare special cases.

Remark 13.12. The condition on the phase of the reference signal in Corollary 13.9 immediately follows from the explicit representation of the second solution in the proof of Theorem 13.4 and the characterization of the solution set in Theorem 5.1. Using a completely different approach, KIM and HAYEs obtain a similar result for real-valued, non-symmetric signals $x$ and $h$, see [KH9ob].

\section{Interference with an unknown reference signal}

After considering additional interference measurements with a known reference signal, the next logical step is to generalize this approach to reference signals $h$ 
that are not known beforehand and must also be recovered. We assume that the corresponding Fourier intensity of $h$ is also given. This leads us to the phase retrieval for two unknown signals $x$ and $h$ with finite support from the three FOURIER intensities

$$
|\mathscr{F}[x]|, \quad|\mathcal{F}[h]|, \quad \text { and }|\mathcal{F}[x+h]| \text {. }
$$

For real-valued signals, this specific phase retrieval problem is examined by KIM and Hayes in [KH93]. Furthermore, a complex version of this problem is considered by RAZ, DuDOvich, and NADLER in [RDN13], where a further interference measurement of the form

$$
|\mathscr{F}[x+\mathrm{i} h]|
$$

besides the three FourIER intensities above is used for the reconstruction of the unknown signals. However, we will show that this fourth FourIER intensity is not necessary to determine the unknown phases even for complex-valued signals $x$ and $h$. More precisely, we can recover the signals $x$ and $h$ up to common trivial ambiguities, which means that both signals can only be rotated, shifted, and reflected and conjugated in exactly the same way. Here we rely on our new representation of all ambiguities of the discrete-time phase retrieval problem in Theorem 5.5.

Theorem 14.1. Let $x$ and $h$ be two discrete-time signals with finite support. If the corresponding zero sets of the signals $x$ and h are disjoint, then both signals $x$ and $h$ can be recovered from the FOURIER intensities

$$
|\mathscr{F}[x]|, \quad|\mathscr{F}[h]|, \quad \text { and }|\mathscr{F}[x+h]|
$$

uniquely up to common trivial ambiguities.

Proof. We begin the proof with the assumption that the considered phase retrieval problem possesses a further different pair of solutions $\breve{x}$ and $\breve{h}$. This means that $\breve{x}$ and $\breve{h}$ fulfil the three equalities

$$
|\mathscr{F}[x]|=|\mathscr{F}[\breve{x}]|, \quad|\mathscr{F}[h]|=|\mathscr{F}[\breve{h}]|, \quad \text { and } \quad|\mathscr{F}[y]|=|\mathscr{F}[\breve{y}]|,
$$

where $y$ and $\breve{y}$ denote the interferences $x+h$ and $\breve{x}+\breve{h}$ respectively.

Obviously, these equations remain valid if we rotate or shift $x$ and $h$ in the same manner, or if we reflect and conjugate both signals. An analogous obser- 
vation holds true for $\breve{x}$ and $\breve{h}$. Therefore, using Theorem 5.5, we always find suitable factorizations of $x$ and $h$ together with appropriate rotations and shifts such that the two solutions of the considered phase retrieval problem can be written as

$$
x=x_{1} * x_{2} \quad \text { and } \quad \breve{x}=\mathrm{e}^{\mathrm{i} \alpha_{1}} \cdot x_{1} * \overline{x_{2}[-\cdot]}
$$

and further

$$
h=h_{1} * h_{2}\left[\cdot-k_{1}\right] \quad \text { and } \quad \breve{h}=\mathrm{e}^{\mathrm{i} \alpha_{2}} \cdot h_{1} * \overline{h_{2}\left[k_{2}-\cdot\right]}
$$

for some real numbers $\alpha_{1}, \alpha_{2}$ and integers $k_{1}, k_{2}$. Starting from the signals $x$ and $h$ with support length $N_{1}$ and $N_{2}$, we can moreover assume that the factors $x_{1}, x_{2}$, $h_{1}$, and $h_{2}$ are discrete-time signals with finite and normalized supports of length $M_{1}, N_{1}-M_{1}, M_{2}$, and $N_{2}-M_{2}$ respectively.

In the next step, we investigate the given interference measurements $|\mathscr{F}[y]|$ and $|\mathcal{F}[\breve{y}]|$. Since $\breve{x}$ and $\breve{h}$ form a further pair of solutions of the considered phase retrieval problem, the interference measurements coincide, and we obtain

$$
|\mathcal{F}[x+h]|^{2}=|\mathcal{F}[\breve{x}+\breve{h}]|^{2}
$$

Together with $|\mathscr{F}[x]|^{2}=|\mathcal{F}[\breve{x}]|^{2}$ and $|\mathcal{F}[h]|^{2}=|\mathcal{F}[\breve{h}]|^{2}$, the expansion of these squared moduli can be simplified to

$$
\mathscr{F}[x] \overline{\mathscr{F}[h]}+\overline{\mathscr{F}[x]} \mathscr{F}[h]=\mathscr{F}[\breve{x}] \overline{\mathscr{F}[\breve{h}]}+\overline{\mathscr{F}[\breve{x}]} \mathscr{F}[\breve{h}] .
$$

Incorporating the FoURIER transform of the convolution representations in (14.1) and (14.2) leads us to

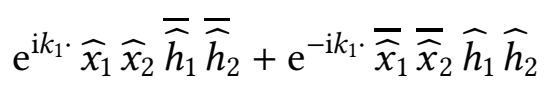

$$
\begin{aligned}
& =\mathrm{e}^{\mathrm{i}\left(\alpha_{1}-\alpha_{2}\right)} \mathrm{e}^{\mathrm{i} k_{2} \cdot \widehat{x}_{1}} \overline{\widehat{x}_{2}} \overline{\widehat{h}}_{1} \widehat{h}_{2}+\mathrm{e}^{-\mathrm{i}\left(\alpha_{1}-\alpha_{2}\right)} \mathrm{e}^{-\mathrm{i} k_{2} \cdot \bar{x}_{1}} \widehat{x}_{2} \widehat{h}_{1} \overline{\widehat{h}}_{2}
\end{aligned}
$$

and finally to

$$
\left[\mathrm{e}^{\mathrm{i} k_{1} \cdot \widehat{x}_{1}} \overline{\widehat{h}}_{1}-\mathrm{e}^{-\mathrm{i}\left(\alpha_{1}-\alpha_{2}\right)} \mathrm{e}^{-\mathrm{i} k_{2} \cdot} \cdot \widehat{\widehat{x}}_{1} \widehat{h}_{1}\right]\left[\widehat{x}_{2} \overline{\widehat{h}}_{2}-\mathrm{e}^{\mathrm{i}\left(\alpha_{1}-\alpha_{2}\right)} \mathrm{e}^{-\mathrm{i}\left(k_{1}-k_{2}\right) \cdot \widehat{\widehat{x}}_{2}} \widehat{h}_{2}\right]=0 .
$$

Since all four discrete-time signals $x_{1}, x_{2}, h_{1}$, and $h_{2}$ possess a finite support, the corresponding FOURIER transforms are trigonometric polynomials. Consequently, we can deduce that either the equality

$$
\widehat{x}_{1} \overline{\widehat{h}}_{1}=\mathrm{e}^{-\mathrm{i}\left(\alpha_{1}-\alpha_{2}\right)} \mathrm{e}^{-\mathrm{i}\left(k_{1}+k_{2}\right) \cdot \overline{\widehat{x}}_{1}} \widehat{h}_{1}
$$


or

$$
\widehat{x}_{2} \overline{\widehat{h}}_{2}=\mathrm{e}^{\mathrm{i}\left(\alpha_{1}-\alpha_{2}\right)} \mathrm{e}^{-\mathrm{i}\left(k_{1}-k_{2}\right) \cdot \overline{\widehat{x}}_{2}} \widehat{h}_{2}
$$

holds everywhere.

Firstly, we assume that the first equality (14.3) holds true. Looking back at the proof of Theorem 5.5, we can note that both factors $x_{1}$ and $h_{1}$ are composed of linear factors from $x$ and $h$. Hence, we find two appropriate subsets of the corresponding zero sets $B:=\left\{\beta_{1}, \ldots, \beta_{N_{1}-1}\right\}$ and $\Gamma:=\left\{\gamma_{1}, \ldots, \gamma_{N_{2}-1}\right\}$ of the original signals $x$ and $h$ such that the factors $x_{1}$ and $h_{1}$ are determined by

$$
\widehat{x}_{1}(\omega)=x_{1}\left[M_{1}-1\right] \prod_{j=1}^{M_{1}-1}\left(\mathrm{e}^{-\mathrm{i} \omega}-\beta_{j}\right)
$$

and

$$
\widehat{h}_{1}(\omega)=h_{1}\left[M_{2}-1\right] \prod_{\ell=1}^{M_{2}-1}\left(\mathrm{e}^{-\mathrm{i} \omega}-\gamma_{\ell}\right) .
$$

Using these factorizations of $x_{1}$ and $h_{1}$ in the frequency domain, we can write equation (14.3) as

$$
\begin{aligned}
& x_{1}\left[M_{1}-1\right] \overline{h_{1}\left[M_{2}-1\right]} \prod_{j=1}^{M_{1}-1}\left(\mathrm{e}^{-\mathrm{i} \omega}-\beta_{j}\right) \prod_{\ell=1}^{M_{2}-1}\left(\mathrm{e}^{\mathrm{i} \omega}-\bar{\gamma}_{\ell}\right) \\
& \quad=\mathrm{e}^{-\mathrm{i}\left(\alpha_{1}-\alpha_{2}\right)} \mathrm{e}^{-\mathrm{i} \omega\left(k_{1}+k_{2}\right)} \overline{x_{1}\left[M_{1}-1\right]} h_{1}\left[M_{2}-1\right] \prod_{j=1}^{M_{1}-1}\left(\mathrm{e}^{\mathrm{i} \omega}-\bar{\beta}_{j}\right) \prod_{\ell=1}^{M_{2}-1}\left(\mathrm{e}^{-\mathrm{i} \omega}-\gamma_{\ell}\right) .
\end{aligned}
$$

Denoting the complex prefactor $x_{1}\left[M_{1}-1\right] \overline{h_{1}\left[M_{2}-1\right]}$ by $C$, and factoring out the conjugated zeros $-\bar{\beta}_{j}$ and $-\bar{\gamma}_{\ell}$ together with the corresponding exponential $\mathrm{e}^{\mathrm{i} \omega}$, we finally obtain the identity

$$
\begin{aligned}
& C \mathrm{e}^{\mathrm{i} \omega\left(M_{2}-1\right)} \prod_{\ell=1}^{M_{2}-1}\left(-\bar{\gamma}_{\ell}\right) \prod_{j=1}^{M_{1}-1}\left(\mathrm{e}^{-\mathrm{i} \omega}-\beta_{j}\right) \prod_{\ell=1}^{M_{2}-1}\left(\mathrm{e}^{-\mathrm{i} \omega}-\bar{\gamma}_{\ell}^{-1}\right) \\
& =\bar{C} \mathrm{e}^{-\mathrm{i}\left(\alpha_{1}-\alpha_{2}\right)} \mathrm{e}^{\mathrm{i} \omega\left(-k_{1}-k_{2}+M_{1}-1\right)} \prod_{j=1}^{M_{1}-1}\left(-\bar{\beta}_{j}\right) \prod_{j=1}^{M_{1}-1}\left(\mathrm{e}^{-\mathrm{i} \omega}-\bar{\beta}_{j}^{-1}\right) \prod_{\ell=1}^{M_{2}-1}\left(\mathrm{e}^{-\mathrm{i} \omega}-\gamma_{\ell}\right) .
\end{aligned}
$$

Using again that both sides of (14.5) are trigonometric polynomials, we can infer that they have the same degree. Consequently, the exponents of the mod- 
ulation factors on both sides must coincide, which yields the identity

$$
M_{2}=M_{1}-k_{2}-k_{1}
$$

Moreover, if we now cancel these modulation factors, we can interpret equation (14.5) as an algebraic polynomial equation in $z:=\mathrm{e}^{-\mathrm{i} \omega}$, where the polynomials on both sides are completely factorized with respect to their zero sets. A comparison of these algebraic polynomials further yields that the zero sets on both sides of (14.5) have to coincide, which means that

$$
\left\{\beta_{1}, \ldots, \beta_{M_{1}-1}\right\} \cup\left\{\bar{\gamma}_{1}^{-1}, \ldots, \bar{\gamma}_{M_{2}-1}^{-1}\right\}=\left\{\bar{\beta}_{1}^{-1}, \ldots, \bar{\beta}_{M_{1}-1}^{-1}\right\} \cup\left\{\gamma_{1}, \ldots, \gamma_{M_{2}-1}\right\} .
$$

Since the corresponding zero sets $B$ and $\Gamma$ are disjoint, and hence the zeros $\beta_{j}$ and $\gamma_{\ell}$ are distinct for all possible indices $j$ and $\ell$, the corresponding zeros of $x_{1}$ and $h_{1}$ not lying on the unit circle must appear in reflected zero pairs of the form

$$
\left(\beta_{j}, \bar{\beta}_{j}^{-1}\right) \text { and }\left(\gamma_{\ell}, \bar{\gamma}_{\ell}^{-1}\right) \text {. }
$$

The corresponding zeros on the unit circle are obviously invariant under reflection at the unit circle, which implies that they can even occur as singletons. Summarizing these observations, we see that the corresponding zero sets of $x_{1}$ and $h_{1}$ are invariant under reflection at the unit circle.

Remembering that both signals $x_{1}$ and $h_{1}$ have a normalized support, we can apply Lemma 5.3 to conclude that the FOURIER transforms of $x_{1}$ and $h_{1}$ are invariant under conjugation except for an additional modulation and rotation. More precisely, Lemma 5.3 implies that the conjugated FouRIER transforms of $x_{1}$ and $h_{1}$ can be represented by

$$
\overline{\widehat{x}}_{1}(\omega)=\overline{x_{1}\left[M_{1}-1\right]} \mathrm{e}^{\mathrm{i} \omega\left(M_{1}-1\right)} \prod_{j=1}^{M_{1}-1}\left(-\bar{\beta}_{j}\right) \prod_{j=1}^{M_{1}-1}\left(\mathrm{e}^{-\mathrm{i} \omega}-\beta_{j}\right)
$$

and

$$
\overline{\widehat{h}}_{1}(\omega)=\overline{h_{1}\left[M_{2}-1\right]} \mathrm{e}^{\mathrm{i} \omega\left(M_{2}-1\right)} \prod_{\ell=1}^{M_{2}-1}\left(-\bar{\gamma}_{\ell}\right) \prod_{\ell=1}^{M_{2}-1}\left(\mathrm{e}^{-\mathrm{i} \omega}-\gamma_{\ell}\right) .
$$

Since the corresponding zeros $\beta_{j}$ and $\gamma_{\ell}$ not lying on the unit circle occur in reflected pairs as discussed above, the absolute values of the products over the corresponding zeros $\left(-\bar{\beta}_{j}\right)$ and $\left(-\bar{\gamma}_{\ell}\right)$ are equal to one. In other words, both products are only unimodular constants and can be described by appropriate rotations. Considering that the conjugation of the leading components $x_{1}\left[M_{1}-1\right]$ 
and $h_{1}\left[M_{2}-1\right]$ can also be interpreted as an additional rotation of the FourIER transforms, we obtain the two identities

$$
\widehat{x}_{1}=\mathrm{e}^{-\mathrm{i} \phi_{1}} \mathrm{e}^{-\mathrm{i}\left(M_{1}-1\right) \cdot \overline{\widehat{x}}_{1}} \quad \text { and } \quad \widehat{h}_{1}=\mathrm{e}^{-\mathrm{i} \phi_{2}} \mathrm{e}^{-\mathrm{i}\left(M_{2}-1\right)} \cdot \overline{\widehat{h}}_{1},
$$

where $\phi_{1}$ and $\phi_{2}$ are real constants that collect the different rotations. For the sake of completeness, the rotations described by the constants $\phi_{1}$ and $\phi_{2}$ are given by

$$
\phi_{1}:=-2 \arg \left(x_{1}\left[M_{1}-1\right]\right)+\arg \left(\prod_{j=1}^{M_{1}-1}\left(-\bar{\beta}_{j}\right)\right)
$$

and

$$
\phi_{2}:=-2 \arg \left(h_{1}\left[M_{2}-1\right]\right)+\arg \left(\prod_{\ell=1}^{M_{2}-1}\left(-\bar{\gamma}_{\ell}\right)\right)
$$

Employing the convolution representations of $x$ and $\breve{x}$ in (14.1) and the representations of $h$ and $\breve{h}$ in (14.2), we can now use the established identities in (14.7) to rewrite the second solution in the frequency domain. We obtain

$$
\widehat{x}=\widehat{x}_{1} \widehat{x}_{2} \quad \text { and } \quad \widehat{\breve{x}}=\mathrm{e}^{\mathrm{i}\left(\alpha_{1}-\phi_{1}\right)} \mathrm{e}^{-\mathrm{i}\left(M_{1}-1\right) \cdot \overline{\bar{x}_{1}}} \overline{\widehat{x}}_{2}
$$

and furthermore

$$
\widehat{h}=\mathrm{e}^{-\mathrm{i} k_{1} \cdot \widehat{h}_{1}} \widehat{h}_{2} \quad \text { and } \quad \widehat{\breve{h}}=\mathrm{e}^{\mathrm{i}\left(\alpha_{2}-\phi_{2}\right)} \mathrm{e}^{-\mathrm{i} \omega\left(M_{2}+k_{2}-1\right)} \overline{\widehat{h}}_{1} \overline{\widehat{h}}_{2} .
$$

Obviously, the solutions $\breve{x}$ and $\breve{h}$ are only trivial ambiguities of $x$ and $h$ caused by rotation, time shift, and reflection and conjugation.

It remains to prove that the rotations and shifts of the trivial ambiguities $\breve{x}$ and $\breve{h}$ coincide. For this purpose, we investigate the leading coefficients on both sides of (14.5). In this manner, we obtain the equality

$$
x_{1}\left[M_{1}-1\right] \overline{h_{1}\left[M_{2}-1\right]} \prod_{\ell=1}^{M_{2}-1}\left(-\bar{\gamma}_{\ell}\right)=\overline{x_{1}\left[M_{1}-1\right]} h_{1}\left[M_{2}-1\right] \mathrm{e}^{-\mathrm{i}\left(\alpha_{1}-\alpha_{2}\right)} \prod_{j=1}^{M_{1}-1}\left(-\bar{\beta}_{j}\right) .
$$

Here the products of the corresponding zeros are again unimodular as discussed above. Next, we describe the conjugations of $x_{1}\left[M_{1}-1\right]$ and $h_{1}\left[M_{2}-1\right]$ once more through additional rotations by $-2 \arg \left(x_{1}\left[M_{1}-1\right]\right)$ and $-2 \arg \left(h_{1}\left[M_{1}-1\right]\right)$ respectively. Neglecting the new common prefactor $x_{1}\left[M_{1}-1\right] h_{1}\left[M_{2}-1\right]$, bringing the factor $\mathrm{e}^{\mathrm{i} \alpha_{2}}$ to the left-hand side, and using the definitions of $\phi_{1}$ and $\phi_{2}$ in (14.8) 
and (14.9), we can simplify the above equation to

$$
\mathrm{e}^{\mathrm{i}\left(\phi_{2}-\alpha_{2}\right)}=\mathrm{e}^{\mathrm{i}\left(\phi_{1}-\alpha_{1}\right)} .
$$

Together with the identity (14.6), we rewrite the representation of $\breve{h}$ in the frequency domain and can finally see that

$$
\widehat{\vec{x}}=\mathrm{e}^{\mathrm{i} \alpha_{1}-\phi_{1}} \mathrm{e}^{-\mathrm{i}\left(M_{1}-1\right) \cdot \overline{\widehat{x}}} \text { and } \widehat{\breve{h}}=\mathrm{e}^{\mathrm{i} \alpha_{1}-\phi_{1}} \mathrm{e}^{-\mathrm{i}\left(M_{1}-1\right) \cdot \overline{\widehat{h}}}
$$

which implies that $\breve{x}$ and $\breve{h}$ exactly arise from the reflection and conjugation of $x$ and $h$ by the same rotation and time shift. Consequently, the second solution pair is only a trivial ambiguity of the original solution pair $x$ and $h$.

If we assume that the equation (14.4) holds true instead of equation (14.3), we can use a completely similar procedure to obtain the representation

$$
\widehat{\breve{x}}=\mathrm{e}^{\mathrm{i}\left(\alpha_{1}+\psi_{1}\right)} \mathrm{e}^{\mathrm{i}\left(N_{1}-M_{1}-1\right) \cdot \widehat{x}} \quad \text { and } \quad \widehat{\breve{h}}=\mathrm{e}^{\mathrm{i}\left(\alpha_{1}+\psi_{1}\right)} \mathrm{e}^{\mathrm{i}\left(N_{1}-M_{1}-1\right) \cdot \widehat{h}}
$$

of the second solution $\breve{x}$ and $\breve{h}$ in the frequency domain, where $\psi_{1}$ is defined like $\phi_{1}$ in (14.8) by using the corresponding zeros of $x_{2}$. Here the second solution pair directly arises from the original signal pair $x$ and $h$ by a common rotation and a common time shift.

In summary, the solution of the considered phase retrieval problem to recover $x$ and $h$ is uniquely solvable up to common trivial ambiguities in both cases (14.3) and (14.4), which leads us to the assertion.

Remark 14.2. The real version of Theorem 14.1 studied by KIM and HaYes in [KH93, Theorem 1] can be established with an analogous argumentation. In doing so, some particularities in the proof of Theorem 14.1 can be avoided by using that all corresponding zeros of a real-valued signal arise in complex conjugate pairs, which simplifies the proof for the real case at some points.

Consequently, we can see the proof of Theorem 14.1 simply as a generalization of the proof in [KH93] to the complex case. Anyway, KIm and HAYEs themselves give the proof only for the special case where both the signal $x$ and the reference signal $h$ do not possess reflected zero pairs or zeros on the unit circle. In this manner, equation (14.5) implies that each of the factors $x_{1}$ and $h_{1}$ is merely a real-valued constant, which immediately finishes the proof.

Remark 14.3. Since the phase retrieval problem considered in Theorem 14.1 is uniquely solvable up to common trivial ambiguities, each further solution pair 
$\breve{x}$ and $\breve{h}$ can be written in the form

$$
\breve{x}:=\mathrm{e}^{\mathrm{i} \alpha} x\left[\cdot-n_{0}\right] \quad \text { and } \quad \breve{h}:=\mathrm{e}^{\mathrm{i} \alpha} h\left[\cdot-n_{0}\right]
$$

or

$$
\breve{x}:=\mathrm{e}^{\mathrm{i} \alpha} \overline{x\left[n_{0}-\cdot\right]} \quad \text { and } \quad \breve{h}:=\mathrm{e}^{\mathrm{i} \alpha} \overline{h\left[n_{0}-\cdot\right]} .
$$

Consequently, in analogy to the phase retrieval problem considered by KIM and HAYES in [KH93, Theorem 1] for two real-valued signals, the interference $x+h$ can also be recovered uniquely up to the same common trivial ambiguity.

Remark 14.4. As mentioned above, RAz et al. consider a similar phase retrieval problem where they wish to recover the two complex-valued signals $x$ and $h$ from the FOURIER intensities

$$
|\mathscr{F}[x]|, \quad|\mathscr{F}[h]|, \quad|\mathscr{F}[x+h]|, \quad \text { and }|\mathscr{F}[x+\mathrm{i} h]| .
$$

This phase retrieval problem is also uniquely solvable up to common trivial ambiguities if the corresponding zero sets of $x$ and $h$ are disjoint, see [RDN13, Theorem 1].

At least theoretically, the fourth FourIER intensity is not required to ensure uniqueness of the phase retrieval problem up to trivial ambiguities as discussed in Theorem 14.1. Anyway, the proof of Theorem 14.1 only shows that the considered phase retrieval problem is uniquely solvable up to trivial ambiguities, but the proof itself is not constructive. Using the fourth FourIER intensity together with the complex polarization identity, RAz et al. give a constructive proof to show that both signals $x$ and $h$ can be recovered analytically.

Although it is very unlikely that the wanted signal and the unknown reference signal possess common corresponding zeros, the question arises: what happens when the corresponding zero sets intersect?

Proposition 14.5. Let $x$ and $h$ be two discrete-time signals with finite support. If the intersection of the corresponding zero sets $B$ and $\Gamma$ of the signals $x$ and $h$ contains a non-empty subset $\Lambda$ such that $\Lambda$ and at least one of the complements $B \backslash \Lambda$ and $\Gamma \backslash \Lambda$ are not invariant under reflection at the unit circle, then the phase retrieval problem to recover $x$ and $h$ from the FOURIER intensities

$$
|\mathscr{F}[x]|, \quad|\mathscr{F}[h]|, \quad \text { and }|\mathscr{F}[x+h]|
$$

possesses at least one further non-trivially different solution pair beside $x$ and $h$. 
Proof. We start from the assumption that the intersection of the corresponding zero sets $B$ and $\Gamma$ contains a non-empty subset $\Lambda$. Consequently, using the characterization in Theorem 5.1, we find a factorization of $x$ and $h$ of the form

$$
\widehat{x}=\widehat{x}_{1} \widehat{y}_{2} \text { and } \widehat{h}=\widehat{h}_{1} \widehat{y}_{2} \text {, }
$$

where the common factor $\widehat{y}_{2}$ corresponds to the corresponding zeros in $\Lambda$.

Based on this specific factorization, we investigate the two discrete-time signals defined by

$$
\widehat{\breve{x}}=\widehat{x}_{1} \overline{\widehat{y}}_{2} \text { and } \quad \widehat{\breve{h}}=\widehat{h}_{1} \overline{\widehat{y}}_{2} \text {. }
$$

In other words, we reflect and conjugate the common convolution factor $y_{2}$ of the original signals $x$ and $h$. Obviously, the FourIER intensities of $x$ and $\breve{x}$ and also the intensities of $h$ and $\breve{h}$ coincide. Hence, the constructed signals satisfy the conditions $|\mathcal{F}[x]|=|\mathscr{F}[\breve{x}]|$ and $|\mathscr{F}[h]|=|\mathscr{F}[\breve{h}]|$. Using the factorization of $\breve{x}$ and $\breve{h}$, we further obtain the equality

$$
|\mathcal{F}[\breve{x}+\breve{h}]|=\left|\widehat{x}_{1}+\widehat{h}_{1}\right|\left|\widehat{\widehat{y}}_{2}\right|=\left|\widehat{x}_{1}+\widehat{h}_{1}\right|\left|\widehat{y}_{2}\right|=|\mathcal{F}[x+h]|,
$$

which implies that the interference measurements also coincide. Hence, the signals $\breve{x}$ and $\breve{h}$ form a further solution pair of the considered phase retrieval problem.

It remains to show that the constructed solution pair is non-trivially different from the first. Since $\Lambda$ is not invariant under reflection at the unit circle, the corresponding zero sets of $\breve{x}$ and $\breve{h}$ cannot coincide with the corresponding zero sets $B$ and $\Gamma$ of the original signals $x$ and $h$. Hence, we can exclude that $\breve{x}$ and $\breve{h}$ are merely rotations or time shifts of $x$ and $h$. Since at least one of the sets $B \backslash \Lambda$ and $\Gamma \backslash \Lambda$ also is not invariant under the reflection, it follows that at least one of the signals $\breve{x}$ and $\breve{h}$ is not a rotation or shift of the corresponding reflected and conjugated original signal. Hence, at least one of the constructed signals is really a non-trivially different ambiguity, which leads us to the assertion.

Remark 14.6. Considering the definition of $\breve{x}$ and $\breve{h}$ in the proof of Proposition 14.5, we see that the constructed signals $\breve{x}$ and $\breve{h}$ possess the corresponding zero sets $B^{(\Lambda)}$ and $\Gamma^{(\Lambda)}$ respectively. Hence, if the subset $\Lambda$ is invariant under reflection at the unit circle, then both modified zero sets $B^{(\Lambda)}$ and $\Gamma^{(\Lambda)}$ coincide with the respective original set $B$ or $\Gamma$, which implies that both signals $\breve{x}$ and $\breve{h}$ are merely trivial ambiguities caused by rotations and time shifts. An analogous argumentation yields that the signals $\breve{x}$ and $\breve{h}$ are rotations and shifts of the reflected and conjugated signals $\overline{x[-\cdot]}$ and $\overline{h[-\cdot]}$ whenever $B \backslash \Lambda$ and $\Gamma \backslash \Lambda$ 
are invariant under reflection. Consequently, the non-empty intersection of the corresponding zero sets $B$ and $\Gamma$ is only a necessary but no sufficient condition to ensure the occurrence of non-trivial ambiguities as stated by RAZ et al. in [RDN13, Theorem 1].

Example 14.7. Let us again consider the signals $x$ and $h$ in Example 13.10, but this time assuming that both signals $x$ and $h$ are unknown. In other words, we try to recover the discrete-time signal

$$
\begin{aligned}
x:=\frac{1}{128}(\ldots, 0, \underline{55-15 i},-84+87 \mathrm{i}, 34+82 \mathrm{i}, \\
204-120 \mathrm{i},-16+16 \mathrm{i},-96,128,0, \ldots)
\end{aligned}
$$

with the corresponding zero set

$$
B=\frac{1}{4}\{1+\mathrm{i}, 3-2 \mathrm{i},-3-\mathrm{i},-4+2 \mathrm{i}, 4+4 \mathrm{i}, 2-4 \mathrm{i}\} .
$$

Further, we choose the discrete-time signal

$$
h:=\frac{1}{64}(\ldots, 0, \underline{0},-22-4 \mathrm{i}, 29-27 \mathrm{i}, 17-59 \mathrm{i}, 6-42 \mathrm{i}, 24,32,0, \ldots)
$$

with the corresponding zero set

$$
\Gamma=\frac{1}{4}\{1+\mathrm{i}, 4+4 \mathrm{i},-4-3 \mathrm{i},-4+2 \mathrm{i},-4 \mathrm{i}\}
$$

to be the 'unknown' reference. The two signals together with the corresponding FOURIER intensities and interference measurements are presented in Figure 14.1 on the following page.

Due to the non-empty intersection $B \cap \Gamma$ of the corresponding zero sets of $x$ and $h$, Proposition 14.5 implies the existence of a further non-trivially different solution. Here we use the subset

$$
\Lambda:=\frac{1}{4}\{1+\mathrm{i}, 4+4 \mathrm{i}\}
$$

of the intersection for the construction of the wanted non-trivial ambiguity. Following the lines in the proof of Proposition 14.5, we obtain a further solution of the considered phase retrieval problem given by the signal

$$
\begin{aligned}
\breve{x}=\frac{1}{128}(\ldots, 0, & ,-30-110 \mathrm{i},-1+68 \mathrm{i}, 69+67 \mathrm{i}, \\
& -72-212 \mathrm{i},-36-52 \mathrm{i},-80+128 \mathrm{i},-64 \mathrm{i}, 0, \ldots)
\end{aligned}
$$




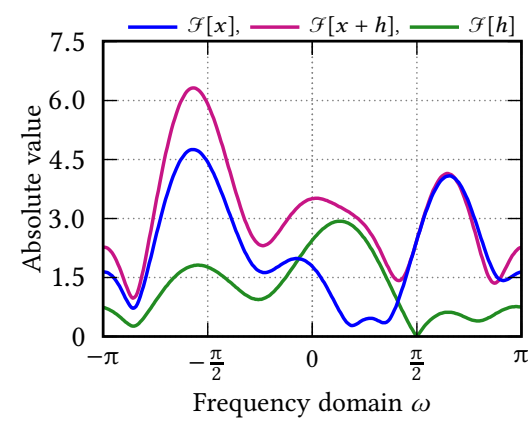

(a) FourIER intensities $\mathscr{F}[x]$ and $\mathscr{F}[h]$ and also the interference measurements $\mathscr{F}[x+h]$ with the unknown reference $h$

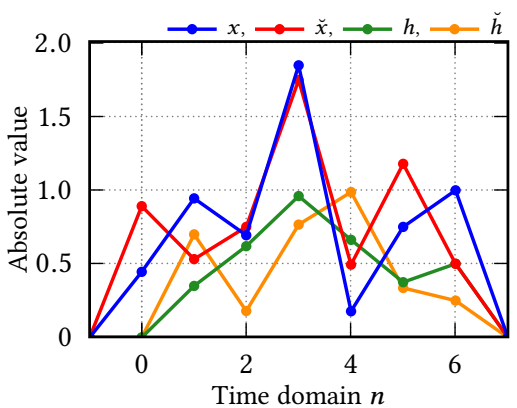

(c) Absolute values of the signals $x$ and $\breve{x}$ and of the reference signals $h$ and $\breve{h}$ illustrated by polygonal lines

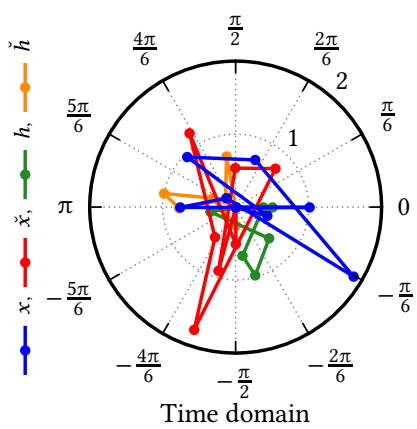

(b) Signals $x$ and $\breve{x}$ together with the reference signals $h$ and $\breve{h}$ represented in polar form

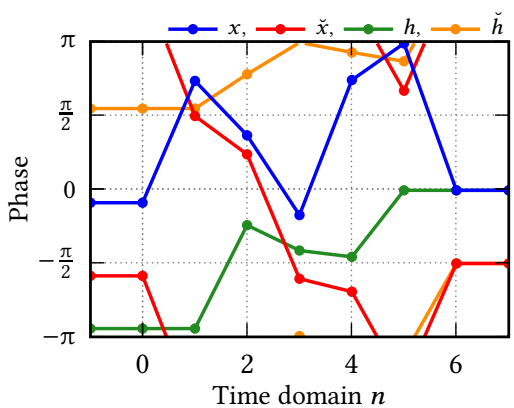

(d) Phases of the signals $x$ and $\breve{x}$ and of the reference signals $h$ and $\breve{h}$ illustrated by polygonal lines

Figure 14.1.: Discrete-time phase retrieval problem to recover the signals $x$ and $h$ from the FourIER intensities $\mathscr{F}[x], \mathscr{F}[h]$, and $\mathscr{F}[x+h]$ with at least one further non-trivially different solution pair $\breve{x}$ and $\breve{h}$. 
and the reference

$$
\breve{h}=\frac{1}{64}(\ldots, 0, \underline{0},-8+44 \mathrm{i},-9+7 \mathrm{i},-49-\mathrm{i},-62+12 \mathrm{i},-20+8 \mathrm{i},-16 \mathrm{i}, 0, \ldots) .
$$

As the subset $\Lambda$ and the two complements $B \backslash \Lambda$ and $\Gamma \backslash \Lambda$ are not invariant under reflection at the unit circle, the constructed solution pair $\breve{x}$ and $\breve{h}$ is nontrivially different from the signals $x$ and $h$, which can also be justified visually by considering Figure 14.1(c) and (d).

Moreover, since the intersection $B \cap \Gamma$ consists of three corresponding zeros, each possible non-empty subset $\Lambda$ of $B \cap \Gamma$ fulfils the requirements of Proposition 14.5. Thus, we could continue this example with the construction of at least $2^{3}=8$ non-trivially different solutions of the considered problem.

\section{Interference with a modulated version of the unknown signal itself}

Next, we examine interference measurements of a slightly different kind. Instead of a known or unknown reference signal $h$, we use the unknown signal $x$ itself as reference and consider interference measurements between $x$ and a modulated version of $x$. For this purpose, we transfer the approaches of CANDÈs, ELDAR, Strohmer, and Voroninski in [CESV13] and Alexeev, Bandeira, Fickus, and MIXoN in [ABFM14] to the discrete-time phase retrieval problem.

More generally, we try to recover the discrete-time signal $x$ with finite support from its FouRIER intensity $|\widehat{x}|$ and a set of interference measurements of the form

$$
\left|\mathcal{F}\left[x+\mathrm{e}^{\mathrm{i} \alpha} \mathrm{e}^{\mathrm{i} \mu \cdot} x\right]\right|
$$

where the modulation and additional rotation are described by the real numbers $\mu$ and $\alpha$. Since the Fourier transform of the modulated signal is obviously given by

$$
\mathcal{F}\left[\mathrm{e}^{\mathrm{i} \alpha} \mathrm{e}^{\mathrm{i} \mu \cdot} x\right](\omega)=\mathrm{e}^{\mathrm{i} \alpha} \sum_{n \in \mathbb{Z}} \mathrm{e}^{-\mathrm{i}(\omega-\mu) n} x[n]=\mathrm{e}^{\mathrm{i} \alpha} \widehat{x}(\omega-\mu),
$$

we can also interpret the considered interference measurements as interferences with certain shifts of the FoURIER transform $\widehat{x}$ in the frequency domain. 


\subsection{Phase reconstruction by using a polarization identity}

With the intent to recover the wanted signal analytically, we firstly apply an appropriate polarization identity to determine the unknown phase in the frequency domain. This idea traces back to Alexeev et al., who introduce the MercedesBENZ polarization identity

$$
\bar{z}_{1} z_{2}=\frac{1}{3} \sum_{k=0}^{2} \mathrm{e}^{\mathrm{i} \frac{2 \pi k}{3}}\left|z_{1}+\mathrm{e}^{-\mathrm{i} \frac{2 \pi k}{3}} z_{2}\right|^{2}
$$

for any complex numbers $z_{1}$ and $z_{2}$ in order to recover a finite-dimensional vector from the intensity measurements of a suitably constructed frame, see [ABFM14]. Denoting by $\zeta_{K}:=\mathrm{e}^{2 \pi \mathrm{i} / K}$ the primitive $K$ th root of unity, we obtain the following generalization.

Lemma 15.1. Let $z_{1}$ and $z_{2}$ be two arbitrary complex numbers. Then the polarization identity

$$
\bar{z}_{1} z_{2}=\frac{1}{K} \sum_{k=0}^{K-1} \zeta_{K}^{k}\left|z_{1}+\zeta_{K}^{-k} z_{2}\right|^{2}
$$

holds for every integer $K>2$.

Proof. The assertion can be proven by generalizing the ideas to justify the original Mercedes-Benz polarization identity (15.1) in [ABFM14, p. 38]. We expand the squared moduli in the sum on the right-hand side and thus obtain

$$
\frac{1}{K} \sum_{k=0}^{K-1} \zeta_{K}^{k}\left|z_{1}+\zeta_{K}^{-k} z_{2}\right|^{2}=\frac{1}{K} \sum_{k=0}^{K-1} \zeta_{K}^{k}\left(\left|z_{1}\right|^{2}+2 \mathfrak{R}\left[\zeta_{K}^{-k} \bar{z}_{1} z_{2}\right]+\left|z_{2}\right|^{2}\right) .
$$

With the well-known identity

$$
\sum_{k=0}^{K-1} \zeta_{K}^{k}=0
$$

for $K>1$, this equality can be simplified to

$$
\frac{1}{K} \sum_{k=0}^{K-1} \zeta_{K}^{k}\left|z_{1}+\zeta_{K}^{-k} z_{2}\right|^{2}=\frac{2}{K} \sum_{k=0}^{K-1} \zeta_{K}^{k} \mathfrak{R}\left[\zeta_{K}^{-k} \bar{z}_{1} z_{2}\right]
$$


Using that the real part of the product $\zeta_{K}^{-k} \bar{z}_{1} z_{2}$ can be written as

$$
\mathfrak{R}\left[\zeta_{K}^{-k} \bar{z}_{1} z_{2}\right]=\mathfrak{R}\left[\zeta_{K}^{-k}\right] \mathfrak{R}\left[\bar{z}_{1} z_{2}\right]-\mathfrak{J}\left[\zeta_{K}^{-k}\right] \mathfrak{J}\left[\bar{z}_{1} z_{2}\right]
$$

together with the identities

$$
\mathfrak{R} \zeta_{K}^{-1}=\mathfrak{R} \zeta_{K}^{k} \text { and } \mathfrak{J} \zeta_{K}^{-k}=-\mathfrak{J} \zeta_{K}^{k}
$$

we can now rearrange the right-hand side of the assertion and find

$$
\frac{1}{K} \sum_{k=0}^{K-1} \zeta_{K}^{k}\left|z_{1}+\zeta_{K}^{-k} z_{2}\right|^{2}=\frac{2}{K} \sum_{k=0}^{K-1} \zeta_{K}^{k}\left(\mathfrak{R}\left[\zeta_{K}^{k}\right] \mathfrak{R}\left[\bar{z}_{1} z_{2}\right]+\mathfrak{J}\left[\zeta_{K}^{k}\right] \mathfrak{J}\left[\bar{z}_{1} z_{2}\right]\right)
$$

Next, we consider the real and imaginary parts separately. Substituting the remaining roots of unity $\zeta_{K}^{k}$ by their algebraic forms $\mathfrak{R} \zeta_{K}^{k}+\mathrm{i} \mathfrak{J} \zeta_{K}^{k}$, we can immediately observe that the real part of the equality is given by

$$
\mathfrak{R}\left[\frac{1}{K} \sum_{k=0}^{K-1} \zeta_{K}^{k}\left|z_{1}+\zeta_{K}^{-k} z_{2}\right|^{2}\right]=\frac{2}{K}\left(\mathfrak{R}\left[\bar{z}_{1} z_{2}\right] \sum_{k=0}^{K-1}\left[\mathfrak{R} \zeta_{K}^{k}\right]^{2}+\mathfrak{J}\left[\bar{z}_{1} z_{2}\right] \sum_{k=0}^{K-1} \mathfrak{R} \zeta_{K}^{k} \mathfrak{J} \zeta_{K}^{k}\right)
$$

and similarly the imaginary part by

$$
\mathfrak{J}\left[\frac{1}{K} \sum_{k=0}^{K-1} \zeta_{K}^{k}\left|z_{1}+\zeta_{K}^{-k} z_{2}\right|^{2}\right]=\frac{2}{K}\left(\mathfrak{R}\left[\bar{z}_{1} z_{2}\right] \sum_{k=0}^{K-1} \mathfrak{J} \zeta_{K}^{k} \mathfrak{R} \zeta_{K}^{k}+\mathfrak{J}\left[\bar{z}_{1} z_{2}\right] \sum_{k=0}^{K-1}\left[\mathfrak{J} \zeta_{K}^{k}\right]^{2}\right) .
$$

If we can show that the sum over the squared real or the squared imaginary parts of all roots of unity is equal to $\mathrm{K} / 2$, and that the sum over the mixed terms is equal to zero, then the assertion follows without circumstances.

Unlike the proof given by Alexeev et al. for the special case where $K$ is equal to three, we cannot evaluate these three sums explicitly. However, remembering that the real and imaginary parts of the roots of unity $\zeta_{K}^{k}$ can be determined by $\mathfrak{R} \zeta_{K}^{k}=1 / 2\left(\zeta_{K}^{-k}+\zeta_{K}^{k}\right)$ and $\mathfrak{J} \zeta_{K}^{k}=\mathrm{i} / 2\left(\zeta_{K}^{-k}-\zeta_{K}^{k}\right)$, and applying the formula for the sum of the first $K$ terms of a geometric series, the required sums can be computed in a simple manner. More precisely, the sum over the squared real parts is given by

$$
\sum_{k=0}^{K-1}\left[\mathfrak{R} \zeta_{K}^{k}\right]^{2}=\frac{1}{4} \sum_{k=0}^{K-1}\left(\zeta_{K}^{-2 k}+2+\zeta_{K}^{2 k}\right)=\frac{K}{2}
$$


the sum over the squared imaginary parts by

$$
\sum_{k=0}^{K-1}\left[\mathfrak{J} \zeta_{K}^{k}\right]^{2}=-\frac{1}{4} \sum_{k=0}^{K-1}\left(\zeta_{K}^{-2 k}-2+\zeta_{K}^{2 k}\right)=\frac{K}{2},
$$

and finally the sum over the real and imaginary parts by

$$
\sum_{k=0}^{K-1} \mathfrak{R} \zeta_{K}^{k} \mathfrak{J} \zeta_{K}^{k}=\frac{i}{4} \sum_{k=0}^{K-1}\left(\zeta_{K}^{-2 k}-\zeta_{K}^{2 k}\right)=0 .
$$

Consequently, the real and imaginary parts of

$$
\frac{1}{K} \sum_{k=0}^{K-1} \zeta_{K}^{k}\left|z_{1}+\zeta_{K}^{-k} z_{2}\right|^{2}
$$

coincide with those of $\bar{z}_{1} z_{2}$, which completes the proof.

Remark 15.2. Obviously, Lemma 15.1 cannot be valid if $K$ is less than three. More detailed, for the excluded cases, we have $\zeta_{1}:=1$ and $\zeta_{2}:=-1$, which implies that the right-hand side of the polarization identity is always a real number. Further, for the special case that $K$ is equal to three, Lemma 15.1 coincides with the original MERCEDES-BENZ polarization identity introduced in [ABFM14, Lemma 2.1].

The generalized polarization identity introduced in Lemma 15.1 can now be used to reveal the relation between the values $\widehat{x}(\omega)$ and $\widehat{x}(\omega-\mu)$ hidden in the interference measurements

$$
\left|\mathcal{F}\left[x+\zeta_{K}^{-k} \mathrm{e}^{\mathrm{i} \mu \cdot} x\right]\right| \quad(k=0, \ldots, K-1)
$$

for some integer $K$ and real number $\mu$. The following theorem shows that the knowledge of this relationship is sufficient to recover each discrete-time signal without any ambiguities.

Theorem 15.3. Let $x$ be a discrete-time signal with finite support of length $N$. If $\mu \neq 2 \pi p / q$ for all $p \in \mathbb{Z}$ and $q \in\{1, \ldots, N-1\}$, then the signal $x$ can be uniquely recovered up to rotation from its FOURIER intensity $|\widehat{x}|$ and the interference measurements

$$
\left|\mathcal{F}\left[x+\zeta_{K}^{-k} \mathrm{e}^{\mathrm{i} \mu \cdot} x\right]\right| \quad(k=0, \ldots, K-1)
$$

for every integer $K$ greater than two. 
Besides the polarization identity, a further key element in the following proof of Theorem 15.3 is the reconstruction of an arbitrary trigonometric polynomial with unknown degree but only a few non-zero coefficients from a small set of known function values. Like in the more general case where one tries to recover a finite exponential sum, this problem can be solved by applying PRONY's method, see for instance [Hil87, Section 9.4] or, for the originally introduced method, [Pro95]. More precisely, Prony's method allows us to recover a finite exponential sum in the following manner, see [Hil87].

Theorem 15.4 (Prony). Let $f$ be the exponential sum given by

$$
f(\omega):=\sum_{n=0}^{N-1} c_{n} \mathrm{e}^{-\mathrm{i} \omega T_{n}}
$$

with non-zero complex coefficients $c_{n}$ and complex frequencies $T_{n}$. If the values $\mathrm{e}^{-\mathrm{i} T_{n}}$ differ pairwise, then the exponential sum $f$ can be completely recovered from the function values at the $2 \mathrm{~N}$ equally spaced points from 0 to $2 \mathrm{~N}-1$.

Remark 15.5. If all frequencies of the exponential sum in Theorem 15.4 are realvalued, then the assumption that the values $\mathrm{e}^{-\mathrm{i} T_{n}}$ differ pairwise means simply that the frequencies do not coincide up to a multiple of $2 \pi$.

Proof of Theorem 15.3. Equipped with the generalized polarization identity and Prony's method, we can now proceed with the proof of Theorem 15.3. For this purpose, we rewrite the given interference measurements

$$
\left|\mathcal{F}\left[x+\zeta_{K}^{-k} \mathrm{e}^{\mathrm{i} \mu \cdot} x\right]\right|=\left|\widehat{x}(\cdot)+\zeta_{K}^{-k} \widehat{x}(\cdot-\mu)\right|
$$

for $k=0, \ldots, K-1$ by applying the polarization identity in Lemma 15.1. In this manner, we obtain the identity

$$
\frac{1}{K} \sum_{k=0}^{K-1} \zeta_{K}^{k}\left|\widehat{x}(\omega)+\zeta_{K}^{-k} \widehat{x}(\omega-\mu)\right|^{2}=\overline{\widehat{x}(\omega)} \widehat{x}(\omega-\mu)
$$

Further, with the polar representation $\widehat{x}=|\widehat{x}| \mathrm{e}^{\mathrm{i} \phi}$, where $\phi$ denotes the phase of $\widehat{x}$, this identity finally becomes

$$
\frac{1}{K} \sum_{k=0}^{K-1} \zeta_{K}^{k}\left|\widehat{x}(\omega)+\zeta_{K}^{-k} \widehat{x}(\omega-\mu)\right|^{2}=|\widehat{x}(\omega)||\widehat{x}(\omega-\mu)| \mathrm{e}^{\mathrm{i}(\phi(\omega-\mu)-\phi(\omega))} .
$$


Dividing this equation by the known FourIER intensity of the signal $x$, we can thus determine the phase difference $\phi(\omega-\mu)-\phi(\omega)$ whenever $\widehat{x}(\omega)$ and $\widehat{x}(\omega-\mu)$ are non-zero.

Since the considered phase retrieval problem can only be solved up to rotations, we can arbitrarily choose the phase $\phi\left(\omega_{0}\right)$ at one point $\omega_{0}$ in the frequency domain without loss of generality. Starting from this point $\omega_{0}$, we can now use the determined phase differences in order to compute further phases of the unknown signal inductively. With the assumption that the FourIER transform $\widehat{x}$ is non-zero at the points $\omega_{0}+\mu k$ for every integer $k$ between 0 and $2 N-1$, we can consequently determine a vector of relative phases

$$
\phi\left(\omega_{0}+\mu k\right)
$$

for $k=0, \ldots, 2 N-1$ with respect to the chosen phase $\phi\left(\omega_{0}\right)$.

Moreover, we can show that there always exists an $\omega_{0}$ such that the FourIER transform $\widehat{x}$ is non-zero at the points $\omega_{0}+\mu k$ for $k$ from 0 to $2 N-1$. Due to the $2 \pi$ periodicity of the FOURIER transform, we can restrict our following observations to the interval $[-\pi, \pi)$. Remembering that the FourIER transform of the signal $x$ is a trigonometric polynomial and can hence be written in the form

$$
\widehat{x}(\omega)=\mathrm{e}^{-\mathrm{i} \omega n_{0}} \sum_{n=0}^{N-1} c_{n} \mathrm{e}^{-\mathrm{i} \omega n}
$$

with a suitable integer $n_{0}$ for the prefactor and an appropriate polynomial in $z=$ $\mathrm{e}^{-\mathrm{i} \omega}$ with complex coefficients $c_{n}$, we can immediately conclude that the FoURIER transform $\widehat{x}$ possesses at most $N-1$ zeros in the interval $[-\pi, \pi)$. Since we can choose the initial point $\omega_{0}$ completely arbitrarily, it is hence always possible to pick an $\omega_{0}$ such that the points

$$
\left(\omega_{0}+\mu k\right) \bmod 2 \pi
$$

do not coincide with the zeros of the FOURIER transform $\widehat{x}$. In other words, we can always inductively construct a series of $2 N$ relative phases.

Up to this point, we have recovered $2 N$ values of the unknown phase $\phi$ and hence of the unknown FOURIER transform $\widehat{x}$ itself. Writing the recovered points of the FOURIER transform (15.2) as

$$
\widehat{x}\left(\omega_{0}+\mu k\right)=\sum_{n=0}^{N-1}\left[c_{n} \mathrm{e}^{-\mathrm{i} \omega_{0}\left(n+n_{0}\right)}\right] \mathrm{e}^{-\mathrm{i} k \mu\left(n+n_{0}\right)},
$$


we can interpret the determined points as function values of the exponential sum with complex coefficients $c_{n} \mathrm{e}^{-\mathrm{i} \omega_{0}\left(n+n_{0}\right)}$ and real frequencies $\mu\left(n+n_{0}\right)$ at the equally spaced points $k$ from 0 to $2 N-1$. In order to apply PronY's method and recover the exponential sum by Theorem 15.4, it remains to show that the real frequencies $\mu\left(n+n_{0}\right)$ for $n$ from 0 to $N-1$ cannot coincide up to a multiple of $2 \pi$, cf. Remark $15 \cdot 5$.

For this purpose, let us assume that we find two frequencies $\mu\left(n_{1}+n_{0}\right)$ and $\mu\left(n_{2}+n_{0}\right)$ with $n_{1}, n_{2} \in\{0, \ldots, N-1\}$ which only differ by a multiple of $2 \pi$. In other words, there exist an integer $\ell$ such that the considered frequencies satisfy the equation

$$
\mu\left(n_{1}+n_{0}\right)=\mu\left(n_{2}+n_{0}\right)+2 \pi \ell .
$$

By an easy rearrangement, we can conclude that this only happens if and only if the parameter $\mu$ is equal to $2 \pi \ell /\left(n_{1}-n_{2}\right)$ for some integer $\ell$. Furthermore, the denominator can only attain non-zero integers between $-N+1$ and $N-1$ because both indicies $n_{1}$ and $n_{2}$ are distinct integers between 0 and $N-1$.

However, since these are exactly the excluded values for the parameter $\mu$ in the assumptions of the theorem, the frequencies $\mu\left(n+n_{0}\right)$ cannot coincide up to a multiple of $2 \pi$; so we can recover the complete exponential sum in (15.3) and hence the complete FourIER transform $\widehat{x}$ from the constructed function values by applying Prony's method, see Theorem 15.4. Finally, we can thus recover the original signal $x$ up to a rotation by using the inverse discrete-time FourIER transform, which justifies the assertion.

Remark 15.6. The main reason for the application of Prony's method in the proof of Theorem 15.3 is the lack of information about the integer $n_{0}$ in the frequency representation (15.2) of the sought signal $x$. Considering the influence of this modulation in the time domain, we only know the support length $N$ of the unknown signal $x$ but not the exact position of the support itself.

If we additionally assume that the signal $x$ in Theorem 15.3 possesses a normalized support, we can recover the FOURIER transform $\widehat{x}$ directly from the constructed function values by solving a linear equation system instead of applying Prony's method because all occurring frequencies in (15.2) are known beforehand. Unfortunately, we cannot neglect the restriction on the parameter $\mu$ in this special case since this restrictions are needed to ensure the invertibility of the arising VANDERMONDE matrix in the new equation system.

Remark 15.7. Considering the assumptions of Theorem 15.3, we have to choose the modulation parameter $\mu$ in a way that $\mu$ is not a rational multiple of $2 \pi$ where the denominator is an integer between 1 and $N-1$. If we now pick the parameter $\mu$ 
as irrational multiple of $2 \pi$, which means that $\mu$ is not contained in $2 \pi \mathbb{Q}$, then we can recover every signal from the given interference measurements independently of the actual support length $N$. Although the support length is needed for the proof of Theorem 15.3, we can skip the assumption that the length $N$ is known beforehand since the actual support length of the signal $x$ is explicitly encoded in the autocorrelation function, which coincides with the given squared FOURIER intensity $|\widehat{x}|^{2}$, see Section 3 .

Remark 15.8. If we assume that the current support of the unknown discretetime signal $x$ with support length $N$ is contained in the set $\{0, \ldots, M-1\}$ with $M \geq N$, which enable us to identify $x$ with an $M$-dimensional vector, then we can interpret the FoURIER intensity $\left|\widehat{x}\left(\omega_{0}\right)\right|$ for a certain point $\omega_{0}$ in the frequency domain as intensity measurement $|\langle x, v\rangle|$ with the frame vector

$$
v:=\left(\mathrm{e}^{\mathrm{i} \omega_{0} m}\right)_{m=0}^{M-1} .
$$

Choosing at least $M$ pairwise different points $\omega_{n}$ in $[-\pi, \pi)$ beforehand, we can consequently apply the whole theory developed by ALEXEEv et al. to recover the unknown vector $x$ from the given intensity measurements $\left|\widehat{x}\left(\omega_{n}\right)\right|$ and the given interference measurements

$$
\left|\widehat{x}\left(\omega_{n}\right)+\zeta_{3}^{-k} \widehat{x}\left(\omega_{m}\right)\right| \quad(k=0,1,2)
$$

for a greater number of randomly chosen index pairs $(n, m)$, see [ABFM14]. More generally, AlExeEv et al. show that the unknown vector can now be recovered with high probability from approximately $240 \mathrm{M}$ measurements for an arbitrary frame, see [ABFM14, p. 41].

In contrast to the findings of AlEXEev et al. for arbitrary frames, we exploit that the FOURIER transform of a discrete-time signal with finite support of length $N$ is a trigonometric polynomial. For the special case $K=3$ considered by Alexeev et al., this enables us to recover the unknown signal $x$ always from merely $8 N-4$ measurements or, more precisely, from $2 N-1$ measurements for each of the four FourIER intensities

$$
|\widehat{x}(\cdot)| \quad \text { and } \quad\left|\widehat{x}(\cdot)+\zeta_{3}^{-k} \widehat{x}(\cdot-\mu)\right| \quad(k=0,1,2),
$$

cf. Remark 3.5. Moreover, the procedure in the proof of Theorem 15.3 allows us to determine the position of the current support from the given FOURIER intensities. In other words, we can recover the unknown signal $x$ without the assumption that the support of $x$ is contained in some specific set $\{0, \ldots, M-1\}$. 


\subsection{Reducing the number of required interference measurements}

Looking back at Theorem 15.3, we observe that the actual number of interference measurements depends on the chosen root of unity $\zeta_{K}$ or, more precisely, on the chosen integer $K$. Consequently, it seems that the given interference measurements are highly redundant. This impression is confirmed by a result in [CESV13], where CANDÈs et al. employ only two of the interference measurements in Theorem 15.3 for the special case that $K$ is a power of two.

In this subsection, we adapt the approach of CANDÈs et al. to the discrete-time phase retrieval problem. Afterwards we generalize this observation and show that each discrete-time signal $x$ can be recovered from its FourIER intensity $|\widehat{x}|$ and two further interference measurements of the form

$$
\left|\mathcal{F}\left[x+\mathrm{e}^{\mathrm{i} \alpha_{1}} \mathrm{e}^{\mathrm{i} \mu \cdot} x\right]\right| \text { and }\left|\mathcal{F}\left[x+\mathrm{e}^{\mathrm{i} \alpha_{2}} \mathrm{e}^{\mathrm{i} \mu \cdot} x\right]\right| \text {, }
$$

where the two rotations $\mathrm{e}^{\mathrm{i} \alpha_{1}}$ and $\mathrm{e}^{\mathrm{i} \alpha_{2}}$ can be chosen nearly arbitrarily. In particular, this rotations do not have to arise from the $K$ th roots of unity for a certain integer $K$.

Theorem 15.9. Let $x$ be a discrete-time signal with finite support of length $N$. If $\mu \neq 2 \pi p / q$ for all $p \in \mathbb{Z}$ and $q \in\{1, \ldots, N-1\}$, then the signal $x$ can be uniquely recovered up to a rotation from its FOURIER intensity $|\widehat{x}|$ and the two interference measurements

$$
\left|\mathcal{F}\left[x+\mathrm{e}^{\mathrm{i} \mu \cdot} x\right]\right| \text { and }\left|\mathcal{F}\left[x-\mathrm{i} \mathrm{e}^{\mathrm{i} \mu \cdot} x\right]\right|
$$

Proof. This theorem can be justified with a similar argument as Theorem 15.3. However, the main difference of the current proof is the determination of the needed relative phases from the two given interference measurements

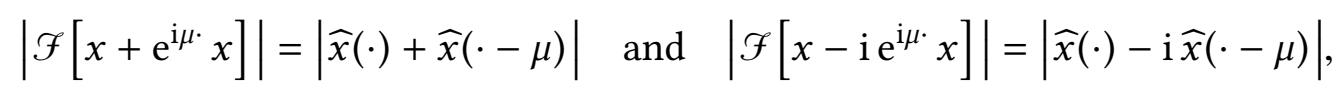

since the generalized polarization identity in Lemma 15.1 can no longer be applied. Instead, we use a completely different approach inspired by CANDÈs et al. in [CESV13, Theorem 3.1]

Expanding the squared interference measurements and writing the FOURIER transform $\widehat{x}$ in the polar form $|\widehat{x}| \mathrm{e}^{\mathrm{i} \phi}$, where $\phi$ denotes unknown phase function, we can stepwise rearrange the given interferences measurements for a certain 
point $\omega$ in the frequency domain and obtain the equalities

$$
\begin{aligned}
\mid \widehat{x}(\omega) & +\left.\widehat{x}(\omega-\mu)\right|^{2} \\
& =|\widehat{x}(\omega)|^{2}+|\widehat{x}(\omega-\mu)|^{2}+[\widehat{x}(\omega) \overline{\widehat{x}(\omega-\mu)}+\overline{\widehat{x}(\omega)} \widehat{x}(\omega-\mu)] \\
& =|\widehat{x}(\omega)|^{2}+|\widehat{x}(\omega-\mu)|^{2}+2 \mathfrak{R}[\overline{\widehat{x}(\omega)} \widehat{x}(\omega-\mu)] \\
& =|\widehat{x}(\omega)|^{2}+|\widehat{x}(\omega-\mu)|^{2}+2|\widehat{x}(\omega)||\widehat{x}(\omega-\mu)| \mathfrak{R}\left[\mathrm{e}^{\mathrm{i}(\phi(\omega-\mu)-\phi(\omega))}\right]
\end{aligned}
$$

and analogously

$$
\begin{aligned}
\mid \widehat{x}(\omega) & -\left.\mathrm{i} \widehat{x}(\omega-\mu)\right|^{2} \\
& =|\widehat{x}(\omega)|^{2}+|\widehat{x}(\omega-\mu)|^{2}+\mathrm{i}[\widehat{x}(\omega) \overline{\widehat{x}(\omega-\mu)}-\overline{\widehat{x}(\omega)} \widehat{x}(\omega-\mu)] \\
& =|\widehat{x}(\omega)|^{2}+|\widehat{x}(\omega-\mu)|^{2}-2 \mathfrak{J}[\overline{\widehat{x}(\omega)} \widehat{x}(\omega-\mu)] \\
& =|\widehat{x}(\omega)|^{2}+|\widehat{x}(\omega-\mu)|^{2}-2|\widehat{x}(\omega)||\widehat{x}(\omega-\mu)| \mathfrak{J}\left[\mathrm{e}^{\mathrm{i}(\phi(\omega-\mu)-\phi(\omega))}\right] .
\end{aligned}
$$

Bringing the squared moduli to the left-hand side, dividing both equations by the known FourIER intensity $|\widehat{x}|$, and combining the obtained real and imaginary part, we can again determine the phase difference $\phi(\omega-\mu)-\phi(\omega)$ whenever $\widehat{x}(\omega)$ and $\widehat{x}(\omega-\mu)$ are non-zero.

At this point, we turn away from the proof given by CANDÈs et al. for a slightly different phase retrieval problem and continue with our observations done in the proof of Theorem 15.3. Like there, we always find a suitable $\omega_{0}$ such that we can inductively construct a series of relative phases

$$
\phi\left(\omega_{0}+\mu k\right)
$$

for $k$ from 0 to $2 N-1$, where the initial phase $\phi\left(\omega_{0}\right)$ can be chosen arbitrarily. Afterwards PRONY's method allows us to recover the complete FouRIER transform $\widehat{x}$ by Theorem 15.4. The theorem can now be established by using the inverse discrete-time FOURIER transform.

Remark 15.10. As mentioned before, CANDÈs et al. consider a slightly different phase retrieval problem in [CESV13, Theorem 3.1]. More precisely, they deal with the problem to recover a finite-dimensional vector from the intensities of its discrete FOURIER transform. Using our notation, we can state this problem as follows: recover the signal $x$ whose support of length $N$ is contained in the 
interval from 0 to $M-1$ from the (discrete-time) FOURIER intensities

$$
\left|\widehat{x}\left(\frac{2 \pi k}{M}\right)\right|, \quad\left|\widehat{x}\left(\frac{2 \pi k}{M}\right)+\widehat{x}\left(\frac{2 \pi(k-\ell)}{M}\right)\right|, \quad \text { and } \quad\left|\widehat{x}\left(\frac{2 \pi k}{M}\right)-\mathrm{i} \widehat{x}\left(\frac{2 \pi(k-\ell)}{M}\right)\right|
$$

for all integers $k=0, \ldots, M-1$ and for a certain integer $\ell$. Under the additional assumption that $\ell$ and $M$ are co-prime, and that the given FourIER samples $|\widehat{x}(2 \pi k / M)|$ are non-zero, CANDÈs et al. show that the unknown signal $x$ can be uniquely recovered.

Recalling that the FOURIER intensity of a discrete-time signal with support length $N$ is entirely determined by $2 N-1$ arbitrary samples, see Remark 3.5, we can directly compare Theorem 15.9 for $\mu:=2 \pi \ell / M$ with the results of CANDÈs et al. in [CESV13, Theorem 3.1] and see that both statements are almost identical. In the special case that $N$ and $M$ coincide, the main difference between both statements is that we need twice as many measurements as CANDÈs et al. Anyway, this enables us to neglect the assumption that the given samples of the FOURIER intensity have to be non-zero. Further, we can determine the unknown position of the current support completely from the given FouRIER intensities.

Finally, the integers $N$ and $M$ have a slightly different meaning. With the dimension $M$, we determine the interval $\{0, \ldots, M-1\}$ that contains the nonzero components of the considered signal $x$. The current support length $N$ of this signal can however be much smaller than the assumed dimension $M$. Consequently, if $M$ is only a rough estimation, then Theorem 15.9 allows us to recover the wanted signal from a much smaller set of measurements than [CESV13, Theorem 3.1].

Based on our observations regarding Theorem 15.9, we can now generalize the result of CANDÈs et al. given in [CESV13, Theorem 3.1] even further. More precisely, we can replace the rotations $1=\mathrm{e}^{0 \cdot \mathrm{i}}$ and $-\mathrm{i}=\mathrm{e}^{-\pi / 2 \cdot \mathrm{i}}$ occurring within the interference measurements by two nearly arbitrary rotations $\mathrm{e}^{\mathrm{i} \alpha_{1}}$ and $\mathrm{e}^{\mathrm{i} \alpha_{2}}$.

Theorem 15.11. Let $x$ be a discrete-time signal with finite support of length $N$. If $\mu \neq 2 \pi p / q$ for all $p \in \mathbb{Z}$ and $q \in\{1, \ldots, N-1\}$, then the signal $x$ can be uniquely recovered up to a rotation from its FOURIER intensity $|\widehat{x}|$ and the two interference measurements

$$
\left|\mathcal{F}\left[x+\mathrm{e}^{\mathrm{i} \alpha_{1}} \mathrm{e}^{\mathrm{i} \mu \cdot} x\right]\right| \text { and }\left|\mathcal{F}\left[x+\mathrm{e}^{\mathrm{i} \alpha_{2}} \mathrm{e}^{\mathrm{i} \mu \cdot} x\right]\right|,
$$

where $\alpha_{1}$ and $\alpha_{2}$ are two real numbers satisfying $\alpha_{1}-\alpha_{2} \neq \pi k$ for all integer $k$. 
Proof. We follow the lines of the proof of Theorem 15.9. Again, the crucial point is the extraction of the required relative phases from the interference measurements

and

$$
\left|\mathcal{F}\left[x+\mathrm{e}^{\mathrm{i} \alpha_{1}} \mathrm{e}^{\mathrm{i} \mu \cdot} x\right]\right|=\left|\widehat{x}(\cdot)+\mathrm{e}^{\mathrm{i} \alpha_{1}} \widehat{x}(\cdot-\mu)\right|
$$

$$
\left|\mathcal{F}\left[x+\mathrm{e}^{\mathrm{i} \alpha_{2}} \mathrm{e}^{\mathrm{i} \mu \cdot} x\right]\right|=\left|\widehat{x}(\cdot)+\mathrm{e}^{\mathrm{i} \alpha_{2}} \widehat{x}(\cdot-\mu)\right|
$$

Replacing the FOURIER transform $\widehat{x}$ by its polar representation $|\widehat{x}| \mathrm{e}^{\mathrm{i} \phi}$, we can rewrite the first interference measurement for a certain point $\omega$ in the frequency domain as

$$
\begin{aligned}
\mid \widehat{x}(\omega) & +\left.\mathrm{e}^{\mathrm{i} \alpha_{1}} \widehat{x}(\omega-\mu)\right|^{2} \\
& =|\widehat{x}(\omega)|^{2}+|\widehat{x}(\omega-\mu)|^{2}+\left[\mathrm{e}^{-\mathrm{i} \alpha_{1}} \widehat{x}(\omega) \overline{\widehat{x}(\omega-\mu)}+\mathrm{e}^{\mathrm{i} \alpha_{1}} \overline{\widehat{x}(\omega)} \widehat{x}(\omega-\mu)\right] \\
& =|\widehat{x}(\omega)|^{2}+|\widehat{x}(\omega-\mu)|^{2}+2|\widehat{x}(\omega)||\widehat{x}(\omega-\mu)| \mathfrak{R}\left[\mathrm{e}^{\mathrm{i}\left(\phi(\omega-\mu)-\phi(\omega)+\alpha_{1}\right)}\right] .
\end{aligned}
$$

Replacing $\alpha_{1}$ by $\alpha_{2}$, we obtain a similar representation for the second interference measurement. Consequently, if both moduli $|\widehat{x}(\omega)|$ and $|\widehat{x}(\omega-\mu)|$ of the Fourier transform $\widehat{x}$ are non-zero, we can determine

$$
\mathfrak{R}\left[\mathrm{e}^{\mathrm{i}\left(\phi(\omega-\mu)-\phi(\omega)+\alpha_{1}\right)}\right] \quad \text { and } \quad \mathfrak{R}\left[\mathrm{e}^{\mathrm{i}\left(\phi(\omega-\mu)-\phi(\omega)+\alpha_{2}\right)}\right] .
$$

In order to extract the phase difference $\phi(\omega-\mu)-\phi(\omega)$ from these two values, we apply EULER's formula and afterwards the addition theorem for cosine. In this manner, we obtain the two equations

$$
\begin{aligned}
\mathfrak{R}\left[\mathrm{e}^{\mathrm{i}\left(\phi(\omega-\mu)-\phi(\omega)+\alpha_{1}\right)}\right]= & \cos \left(\alpha_{1}\right) \cos (\phi(\omega-\mu)-\phi(\omega)) \\
& -\sin \left(\alpha_{1}\right) \sin (\phi(\omega-\mu)-\phi(\omega))
\end{aligned}
$$

and

$$
\begin{aligned}
\mathfrak{R}\left[\mathrm{e}^{\mathrm{i}\left(\phi(\omega-\mu)-\phi(\omega)+\alpha_{2}\right)}\right]= & \cos \left(\alpha_{2}\right) \cos (\phi(\omega-\mu)-\phi(\omega)) \\
& -\sin \left(\alpha_{2}\right) \sin (\phi(\omega-\mu)-\phi(\omega)) .
\end{aligned}
$$

Since the values on the left-hand side are known, we can consequently determine the sine and cosine of the wanted phase difference $\phi(\omega-\mu)-\phi(\omega)$ by solving a simple linear equation system.

In order to investigate the solvability of this equation system, we compute the determinant of the appearing matrix. Here, with the aid of the addition theorem 
for sine, the determinant can be written as

$$
\operatorname{det}\left(\begin{array}{c}
\cos \alpha_{1}-\sin \alpha_{1} \\
\cos \alpha_{2}-\sin \alpha_{2}
\end{array}\right)=\sin \alpha_{1} \cos \alpha_{2}-\cos \alpha_{1} \sin \alpha_{2}=\sin \left(\alpha_{1}-\alpha_{2}\right),
$$

which enforces a unique solution whenever $\alpha_{1}-\alpha_{2}$ does not coincide with a multiple of $\pi$. Consequently, we can always determine the required phase difference $\phi(\omega-\mu)-\phi(\omega)$ for a certain $\omega$.

With the extracted phase difference, the theorem can now be justified as discussed in the proof of Theorem 15.3. Beginning with an appropriate point $\omega_{0}$ together with an arbitrarily chosen phase $\phi\left(\omega_{0}\right)$, we can construct a vector of relative phases $\phi\left(\omega_{0}+\mu k\right)$ for $k=0, \ldots, 2 N-1$, which enables us to recover the Fourier transform $\widehat{x}$ by PrONY's method in Theorem 15.4. Finally, the inverse discrete-time FourIER transform leads us to the assertion. 



\title{
Continuous-time phase retrieval
}

\begin{abstract}
Dissimilar from our previous observations, we will now extend our investigation to the continuous-time setting. Inspired by the phase retrieval of linear spline functions with equally spaced knots introduced in [SSD ${ }^{+} \mathrm{o6}, \mathrm{LTo}$ ], we will firstly restrict ourselves to the recovery of structured signals. We will see that the phase retrieval problem of structured signals has the same behavior like the discrete-time problem, which will allow us to transfer our previous findings to the new setting. For the phase retrieval problem of continuous-time signals without any structure, we will state the characterization of all occurring ambiguities given by Hofstetter [Hof64]. With the help of this characterization, we will examine the relationship between the discrete-time and continuous-time problem. Finally, we will transfer the approach to exploit additional interference measures to the continuous-time setting. Here, we will give a series of novel results, which show that continuous-time signals can be uniquely recovered by using interferences with an unknown reference signal or with a modulated versions of the unknown signal itself.
\end{abstract}

${ }^{a}$ Some of the observations in this chapter have been published in [BP $\left.15 \mathrm{~b}\right]$.

\section{Recovery of structured continuous-time signals}

Quite different from the chapters before, we now leave the discrete-time phase retrieval problem and consider the continuous-time setting. As the name implies, the continuous-time phase retrieval problem describes the problem to recover an unknown continuous-time signal or, mathematically, a complex-valued function under suitable assumptions from its FourIER intensity. The main motivations, which lead us through this chapter, are the following two questions. Firstly, how is the discrete-time problem related to the continuous-time case, and secondly, how far can we generalize our previous findings to the new setting? 
We begin our investigations of the continuous-time phase retrieval problem with the consideration of a special class of signals which simultaneously combines the properties and characteristics of the discrete-time and continuous-time settings. More precisely, we examine the phase retrieval of an unknown structured signal or structured function $f: \mathbb{R} \rightarrow \mathbb{C}$ of the form

$$
f(t):=\sum_{n \in \mathbb{Z}} c[n] \varphi(t-n),
$$

where $\varphi$ is an a priori given generator function not equal to zero, and where $c:=(c[n])_{n \in \mathbb{Z}}$ is a complex-valued sequence with finite support.

Similarly to the discrete-time FoURIER transform in Section 1; for a continuous-time signal $f: \mathbb{R} \rightarrow \mathbb{C}$, we here employ the (continuous-time) FOURIER transform defined by the integral

$$
\mathcal{F}[f](\omega):=\widehat{f}(\omega):=\int_{-\infty}^{\infty} f(t) \mathrm{e}^{\mathrm{i} \omega t} \mathrm{~d} t .
$$

In order to ensure that the FouRIER transform $\mathscr{F}[f]$ itself is a well-defined function, we assume in the following that the complex-valued signal $f$ is an absolutely integrable function in $L^{1}$ or a square-integrable function in $L^{2}$, see for instance [SW71, Chapter I]. Firstly, we consider the following special case of the continuous-time phase retrieval problem.

Problem 16.1. The continuous-time phase retrieval problem for structured signals is the problem of recovering a continuous-time signal $f$ of the form (16.1) with a generator function $\varphi$ in $L^{1}$ or $L^{2}$ from its FourIER intensity $|\mathcal{F}[f]|$.

For a specifically chosen generator function $\varphi$, phase retrieval problems of this kind are introduced in [SSD ${ }^{+}$o6, LTo8, LTo9]. For example, if we choose the centred linear B-spline defined by

$$
\varphi(t):= \begin{cases}1-|t| & t \in[-1,1] \\ 0 & \text { else }\end{cases}
$$

as generator function $\varphi$, then the structured signal $f$ in (16.1) is nothing but a linear spline function. With the intent to determine a solution numerically, SEIFERT et al. [SSD ${ }^{+}$o6] and LANGEMANN and TASCHE [LTo8] examine the corresponding phase retrieval problem. 
Similarly to the discrete-time version, the phase retrieval problem for structured signals (Problem 16.1) cannot be solved uniquely. For example, we can easily construct further solutions by rotating or shifting an already known solution. Moreover, if the generator function $\varphi$ is invariant under reflection and conjugation, then reflecting and conjugating a solution of Problem 16.1 also yields a further solution. More generally, rotation, time shift by an integer, and reflection and conjugation of a given solution always results in an ambiguity of the considered problem, which can be proven like Proposition 2.1. For this reason, we again distinguish between trivial ambiguities (caused by rotation, shift, and reflection and conjugation) and non-trivial ambiguities.

With the intent to characterize all occurring ambiguities in Problem 16.1, we briefly consider the FouRIER transform of the structured signal (16.1), which is determined by

$$
\widehat{f}(\omega)=\widehat{\varphi}(\omega) \sum_{n \in \mathbb{Z}} c[n] \mathrm{e}^{-\mathrm{i} \omega n}
$$

Apart from the FourIER transformed generator function $\widehat{\varphi}$, we can here observe that the FourIER transform is dominated by a trigonometric series, which gathers the time shifts of the generator $\varphi$. More precisely, the obtained series is nothing but the discrete-time FourIER transform of the coefficient sequence $c$ of the considered structured signal $f$. This simple observation allows us to transfer the characterization of all ambiguities occurring in the discrete-time phase retrieval problem to the current setting.

Theorem 16.2. Let $f$ be a structured signal of the form (16.1) whose coefficient sequence $c$ has a finite support length $N$, and whose generator function $\varphi$ is contained in $L^{1}$ or $L^{2}$. Then the FOURIER transform of each structured signalg of the same form satisfying $|\mathcal{F}[g]|=|\mathcal{F}[f]|$ can be written as

$$
\widehat{g}(\omega)=\mathrm{e}^{\mathrm{i}\left(\alpha-\omega n_{0}\right)} \widehat{\varphi}(\omega) \sqrt{|a[N-1]| \prod_{j=1}^{N-1}\left|\beta_{j}\right|^{-1}} \cdot \prod_{j=1}^{N-1}\left(\mathrm{e}^{-\mathrm{i} \omega}-\beta_{j}\right),
$$

where $\alpha$ is a real number, $n_{0}$ is an integer, $a$ is the autocorrelation signal to the coefficients sequence c, and $\beta_{j}$ is chosen from the zero pair $\left(\gamma_{j}, \bar{\gamma}_{j}^{-1}\right)$ of the associated polynomial to $|\widehat{c}|^{2}$.

Proof. We begin the proof with the assumption that $g$ is a further structured signal of the form (16.1) whose FouRIER intensity coincide with the FoURIER in- 
tensity of the given structured signal $f$. Consequently, we always find an appropriate coefficient sequence $b$ such that $g$ can be written as

$$
g(t):=\sum_{n \in \mathbb{Z}} b[n] \varphi(t-n)
$$

Moreover, extending the FOURIER intensities of $f$ and $g$, we have the pointwise equality

$$
|\widehat{f}(\omega)|^{2}=|\widehat{\varphi}(\omega)|^{2}\left|\sum_{n \in \mathbb{Z}} c[n] \mathrm{e}^{-\mathrm{i} \omega n}\right|^{2}=|\widehat{\varphi}(\omega)|^{2}\left|\sum_{n \in \mathbb{Z}} b[n] \mathrm{e}^{-\mathrm{i} \omega n}\right|^{2}=|\widehat{g}(\omega)|^{2}
$$

for almost every $\omega$ in the frequency domain.

Due to the assumption that the generator function $\varphi$ and hence the FourIER transform $\widehat{\varphi}$ are not constantly zero, we find at least a small interval $I$ where $\widehat{\varphi}$ is non-zero almost everywhere, cf. [Coh8o, Proposition 1.4.8]. Dividing the squared FourIER intensities by $|\widehat{\varphi}|^{2}$, we thus obtain the pointwise equality

$$
|\widehat{c}(\omega)|^{2}=\left|\sum_{n \in \mathbb{Z}} c[n] \mathrm{e}^{-\mathrm{i} \omega n}\right|^{2}=\left|\sum_{n \in \mathbb{Z}} b[n] \mathrm{e}^{-\mathrm{i} \omega n}\right|^{2}=|\widehat{b}(\omega)|^{2}
$$

with the discrete-time FoURIER transformed coefficient sequences $\widehat{c}$ and $\widehat{b}$ for almost every $\omega$ in $I$. However, since the squared FourIER intensities on both sides are trigonometric polynomials, we can continuously extend this equality to the entire interval $I$ and afterwards to the complete frequency domain.

The coefficient sequence $b$, which completely determines the structured signal $g$, thus has to satisfy the equation $|\mathscr{F}[c]|=|\mathscr{F}[b]|$. Interpreting the coefficient sequences $c$ and $b$ as discrete-time signals, we can now apply the characterization in Theorem 5.1 to conclude that the discrete-time FourIER transform of the sequence $b$ can be written in the form

$$
\widehat{b}(\omega)=\mathrm{e}^{\mathrm{i}\left(\alpha-\omega n_{0}\right)} \sqrt{|a[N-1]| \prod_{j=1}^{N-1}\left|\beta_{j}\right|^{-1}} \cdot \prod_{j=1}^{N-1}\left(\mathrm{e}^{-\mathrm{i} \omega}-\beta_{j}\right)
$$

with a real number $\alpha$, an integer $n_{0}$, and corresponding zeros $\beta_{j}$ chosen from the zero pairs $\left(\gamma_{j}, \bar{\gamma}_{j}^{-1}\right)$ of the associated polynomial to $|\widehat{c}|^{2}$. Moreover, $a[N-1]$ here denotes the leading coefficient of the autocorrelation signal to the coefficient sequence $c$. Considering the Fourier transform $\widehat{\varphi}$ of the generator function immediately leads to the assertion. 
Remark 16.3. The main idea of the proof of Theorem 16.2 is to reduce the considered continuous-time phase retrieval problem for structured signals to a discrete-time phase retrieval problem. To determine the required discrete-time FourIER intensity of the coefficient sequence $c$, we employ the given FourIER intensity $\mathscr{F}[f]$ over an entire interval. In other words, we make use of infinitely many function values.

Especially for an arbitrary generator function $\varphi$ in $L^{2}$, this approach is necessary since the FourIER transform $\widehat{\varphi}$ is again a square-integrable function in $L^{2}$ and hence only determined up to a set with zero LEBEsGue measure. Consequently, without further appropriate restrictions, the actual value of $\widehat{\varphi}(\omega)$ for a certain $\omega$ in the frequency domain is not well defined and cannot be employed directly.

Anyway, if we assume that the generator function is an absolutely integrable function in $L^{1}$, then the Fourier transform $\widehat{\varphi}$ is uniformly continuous, see for instance [SW71, Theorem 1.1]. Thus, the FouRIER transform is uniquely defined everywhere, and we can make use of the function values $\widehat{\varphi}(\omega)$ themselves. Since the discrete-time Fourier transform of the coefficient sequence $c$ is also continuous, we can completely determine the required FourIER intensity $|\widehat{c}|$ from only $2 N-1$ samples of the known quotient $|\mathscr{f}[f]|^{2} /|\mathcal{F}[\varphi]|^{2}$ at appropriate points, see Remark 3.5.

Remark 16.4. Different from Theorem 5.1, we have characterized the ambiguities of the phase retrieval problem for a structured signal by Theorem 16.2 in the frequency domain. The reason for this approach is that the linear factors $\mathrm{e}^{-\mathrm{i} \omega}-\beta_{j}$ are neither absolutely integrable nor square-integrable, and that the (inverse) Fourier transform of these factors thus is not covered by the $L^{1}$ or $L^{2}$ theory.

Using the theory of distributions, we can nevertheless transfer the characterization to the time domain. For this purpose, we denote by $\delta_{0}$ the Dirac delta distribution, whose Fourier transform can be interpreted as the constant function identical to one. Consequently, the inverse FourIER transform of the exponential $\mathrm{e}^{\mathrm{i} \omega n_{0}}$, which is nothing but a modulation of the constant function, is a shifted version of the DiRAC delta distribution. Similar to the discrete-time setting, we denote the shifted Dirac delta distribution by $\delta_{n_{0}}$.

In this manner, we can thus determine the inverse Fourier transform $\widehat{g}$ in Theorem 16.2 by applying the convolution theorem for distributions and obtain the characterization

$$
g=\mathrm{e}^{\mathrm{i} \alpha} \sqrt{|a[N-1]| \prod_{j=1}^{N-1}\left|\beta_{j}\right|^{-1}} \cdot\left[\varphi * \delta_{n_{0}} * \underset{j=1}{*} \underset{*}{*-1}\left(\delta_{1}-\beta_{j} \delta_{0}\right)\right],
$$


of all occurring ambiguities of the phase retrieval problem for structured signals (Problem 16.1) in the time domain.

Although the phase retrieval problem for structured signals is located in the continuous-time setting, the ambiguousness of Problem 16.1 only depends on the appearing ambiguities of the discrete-time phase retrieval problem to recover the coefficient sequence. This behaviour can especially be observed within the characterization of all possible solutions in the time domain, see Remark 16.4. Considering equation (16.2), we can interpret an arbitrary structured signal as convolution of the generator function $\varphi$ with an appropriate impulse train or a suitable discrete-time signal. Moreover, all occurring ambiguities in the recovery of a structured signal can be obtained by convolving the generator function with the solutions of the discrete-time phase retrieval problem to recover these impulse trains.

Example 16.5. Intending to give an explicit instance of the continuous-time phase retrieval problem for a structured signal, we try to recover a sum of shifted GAUssian functions from its FourIER intensity. More detailed, we here assume that the generator function $\varphi$ and hence the FOURIER transform $\widehat{\varphi}$ are GAUssian functions of the form

$$
\varphi(t):=\mathrm{e}^{-\frac{t^{2}}{2}} \text { and } \widehat{\varphi}(\omega)=\sqrt{2 \pi} \mathrm{e}^{-\frac{\omega^{2}}{2}}
$$

respectively, see for instance [DM12, p. 132].

For the coefficient sequence $c$ of the resulting structured signal

$$
f(t):=\sum_{n \in \mathbb{Z}} c[n] \mathrm{e}^{-\frac{(x-n)^{2}}{2}},
$$

we again employ the discrete-time signal examined in Example 1.1. We choose the coefficient sequence $c$ of the structured signal $f$ as

$$
\begin{aligned}
c:=\frac{1}{128}(\ldots, 0, \underline{55-15 i},-84+87 i, 34+82 \mathrm{i}, \\
204-120 \mathrm{i},-16+16 \mathrm{i},-96,128,0, \ldots) .
\end{aligned}
$$

In this manner, we obtain the continuous-time signal $f$ and the FoURIER intensity $|\mathscr{F}[f]|$ illustrated in Figure 16.1 on the next page.

Looking back at Theorem 16.2, we can now construct all further non-trivial solutions of the considered phase retrieval problem by reflecting some of the 


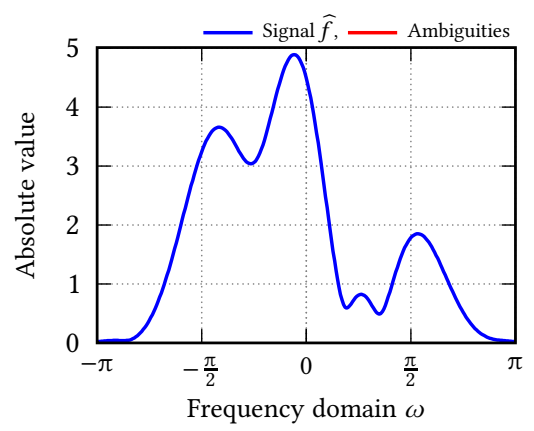

(a) Fourier intensities of the signal $f$ and all non-trivial ambiguities

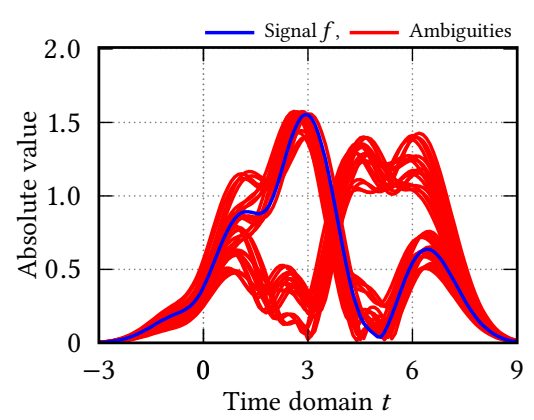

(c) Absolute value of the signal $f$ and all non-trivial ambiguities

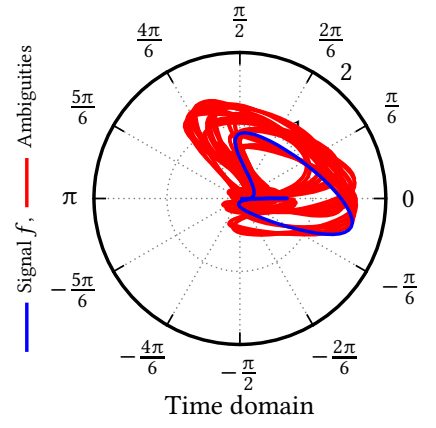

(b) Signal $f$ and all non-trivial ambiguities represented in polar form

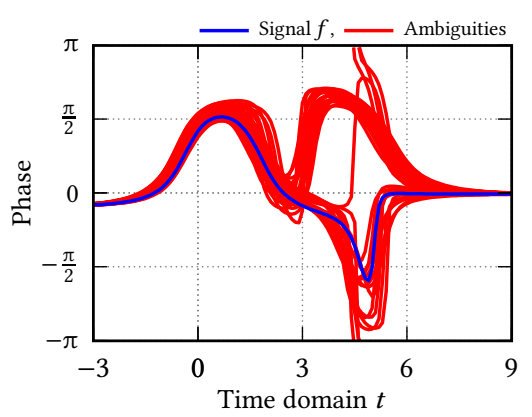

(d) Phase of the signal $x$ and all nontrivial ambiguities

Figure 16.1.: Continuous-time phase retrieval problem for structured signals with a full solution set of $2^{5}$ non-trivial ambiguities

corresponding zeros $\beta_{j}$ in

$$
B=\frac{1}{4}\{1+\mathrm{i}, 3-2 \mathrm{i},-3-\mathrm{i},-4+2 \mathrm{i}, 4+4 \mathrm{i}, 2-4 \mathrm{i}\}
$$

at the unit circle similarly to the discrete-time setting. The reflection of the entire corresponding zero set again results in the reflection and conjugation of a certain solution since our generator function is conjugate symmetric. Thus, we can find exactly $2^{5}=32$ further non-trivially different solutions. Graphically, these nontrivial ambiguities are also presented in Figure 16.1. 


\section{Avoiding ambiguousness in the phase retrieval of struc- tured signals}

The close relation between the discrete-time phase retrieval problem and the continuous-time problem for structured signals allows us to transfer most of our findings in the previous chapters to the new setting without circumstances. For example, the number of possible non-trivially different solutions can be determined as in Proposition 6.1. Nevertheless, some results, especially our investigations of phase retrieval problems with a priori restrictions or additional data in the time domain, cannot be adopted straightforwardly because an arbitrary generator function in $L^{1}$ or $L^{2}$ does not allow the direct utilization of individual function values or the hidden coefficient sequence.

To overcome these difficulties, we restrict ourselves if necessary to structured signals (16.1) with an appropriately chosen generator function $\varphi$. On the one hand, we assume that the generator function $\varphi$ is continuous everywhere, which avoids that the generator $\varphi$ is merely defined up to a set with zero LEBESGUE measure and allows us to examine additional data from individual points in the time domain. On the other hand, we assume that the generator function $\varphi$ is a LAGRANGE function. In other words, we assume that $\varphi$ fulfils the LAGRANGE condition

$$
\varphi(n)=\delta_{0 n}:= \begin{cases}1 & n=0 \\ 0 & \text { else }\end{cases}
$$

for every integer $n$, where $\delta_{0 n}$ denotes the Kronecker delta, see for instance [Buho3, p. 51 et seq.]. In this manner, we directly have access to the coefficients of the structured signal by

$$
f(k)=\sum_{n \in \mathbb{Z}} c[n] \varphi(k-n)=c[k]
$$

for every integer $k$.

For some statements, especially in Chapter II and Chapter III, we have identified the considered discrete-time signal with a real-valued or complex-valued finite-dimensional vector by normalizing the support of the signal. For structured signal (16.1), we pursue a similar approach. Since a time shift of a structured signal $f$ by an integer, which is equivalent to a shift of the coefficient sequence $c$, always results in a further solution of the problem, we can normalize the support of the coefficient sequence $c$ to the index set $\{0, \ldots, N-1\}$ without loss of generality. Here $N$ denotes the support length of the coefficient sequence $c$. In 
other words, we assume that the unknown structured signal $f$ can be written as

$$
f(t):=\sum_{n=0}^{N-1} c[n] \varphi(t-n)
$$

for an appropriate generator function $\varphi$.

Since the time shifts of the generator function $\varphi$ are always linearly independent, the structured signals of the form (17.2) with normalized coefficient sequences form a complex or real $\mathrm{N}$-dimensional vector space. More detailed, the linear independence follows by considering an arbitrary structured signal (17.2) equal to zero and its FouRIER transform. In this manner, we obtain the two identities

$$
\sum_{n=0}^{N-1} c[n] \varphi(\cdot-n) \equiv 0 \quad \text { and } \quad \widehat{\varphi} \sum_{n=0}^{N-1} c[n] \mathrm{e}^{-\mathrm{i} n \cdot} \equiv 0 .
$$

Similarly to the proof of Theorem 16.2, we can always determine the trigonometric polynomial appearing in the frequency domain and hence its coefficients $c[n]$. Since the trigonometric system $\left\{\mathrm{e}^{-\mathrm{i} n \cdot}: n \in \mathbb{Z}\right\}$ is an orthonormal set over $[-\pi, \pi)$ and therefore linearly independent, see for instance [Rud7o, p. 89], the entire coefficient sequence has to be zero. In other words, we can never describe the generator function $\varphi$ by a non-trivial linear combination of finitely many translations. Consequently, we can identify a structured signal with its unique coefficient sequence and hence again with an $\mathrm{N}$-dimensional or $2 \mathrm{~N}$-dimensional real vector according to whether the considered functions are real-valued or complex-valued.

After these preliminary considerations, we are now ready to transfer our previous observations for the discrete-time phase retrieval problem to the recovery of structured signals. Here we begin with the main result of Chapter II and consider a structured signal with non-negative real generator function and realvalued coefficient sequence.

Corollary 17.1. Let $\varphi$ be a non-negative and symmetric real-valued generator function in $C^{0} \cap L^{1}$ or $C^{0} \cap L^{2}$ fulfilling the LAGRANGE condition, and let $c$ be a realvalued coefficient sequence with normalized support of length $N$ with $N>3$. Then the structured signals (17.2) that can be recovered uniquely up to reflection as well as the signals that cannot be recovered uniquely up to reflection from their FOURIER intensities are unbounded sets containing a cone of infinite LEBESGUE measure. 
Proof. Due to the assumption that the generator function $\varphi$ is a continuous LAGRANGE function, the components of the coefficient sequence $c$ coincide with certain function values of the wanted structured signal. More precisely, the components are directly given by $c[n]=f(n)$ for all integers $n$ as discussed in (17.1). Consequently, the structured signal $f$ is non-negative if and only if the coefficient sequence is non-negative.

Considering that the Fourier intensity $|\widehat{c}|$ of the coefficient sequence can be computed from the given intensity $|\mathscr{F}[f]|$ as shown in the proof of Theorem 16.2, we can reduce the entire problem to the phase retrieval of the unknown nonnegative sequence $c$ from its FourIER intensity. Now, the assertion simply follows from Theorem 9.15.

Remark 17.2. Obviously, the reflection of a non-negative structured signal is non-negative too. However, if the generator function $\varphi$ is not an even function, then this reflection cannot be written in the form (16.2), which implies that the reflected signal is not a valid solution of the considered problem. The reflection of the coefficient sequence $c$ is nevertheless a solution of the underlying discretetime phase retrieval problem. Starting from a non-symmetric generator function $\varphi$, we hence always find a second non-trivially different solution corresponding to the reflected coefficient sequence.

Remark 17.3. If the centred linear B-spline is chosen as generator function $\varphi$, we obtain the phase retrieval problem to recover a non-negative linear spline function from its continuous-time FOURIER intensity. This specific phase retrieval problem was introduced by SEIFERT et al. in [SSD ${ }^{+}$o6], who developed a method to solve this problem numerically.

The additional restriction that a real-valued structured signal is non-negative can again reduce the set of occurring non-trivial ambiguities but cannot enforce the uniqueness in general. Like for the discrete-time analogue, we can observe that neither the uniqueness nor the ambiguousness is a rare exception. We now return to the phase retrieval problem of complex-valued structured signals and assume that we have additional access to at least one absolute value or two phases in the time domain. Adapting the main results in Chapter III, we obtain the following two corollaries.

Corollary 17.4. Let $\varphi$ be a complex-valued generator function in $C^{0} \cap L^{1}$ or $C^{0} \cap L^{2}$ fulfilling the LAGRANGE condition, let $c$ be a complex-valued coefficient sequence with normalized support of length $N$, and let $\ell$ be an arbitrary integer between 0 
and $N-1$. Then the phase retrieval problem to recover the structured signal in (17.2) from its FOURIER intensity $|\mathcal{F}[f]|$ and the absolute value $|f[N-1-\ell]|$ is almost always uniquely solvable up to rotations whenever $\ell \neq(N-1) / 2$. In the special case that $\ell=(N-1) / 2$, the reconstruction is only unique up to rotation and conjugate reflection of the coefficient sequence $c$.

Proof. Since the generator function $\varphi$ satisfies the LAGRAnge condition, we can use (17.1) to obtain the identity

$$
|c[N-1-\ell]|=|f[N-1-\ell]| .
$$

Determining the FourIER intensity $|\widehat{c}|$ from the given intensity $|\mathcal{F}[f]|$ as discussed in the proof of Theorem 16.2, we can thus reduce the considered problem to the recovery of the coefficient sequence $c$ from its discrete-time FoURIER intensity $|\widehat{c}|$ and the additionally given moduli $|c[N-1-\ell]|$. Now, applying Theorem 10.11 yields the assumption.

Remark 17.5. If we have access to the moduli $|f(n)|$ for every integer $n$, Corollary 17.4 obviously remains valid. Choosing the centred linear B-spline as generator function as mentioned above, we exactly obtain the phase retrieval problem investigated by SEIFERT et al. in $\left[\mathrm{SSD}^{+} \mathrm{o6}\right.$ ] or LANGEMANN and TASCHE in [LTo8]. Moreover, in [LTo9], LANGEMANN and TASCHE adapt their developed numerical methods to a specifically constructed LAGRANGE function.

Corollary 17.6. Let $\varphi$ be a complex-valued generator function in $C^{0} \cap L^{1}$ or $C^{0} \cap L^{2}$ fulfilling the LAGRANGE condition, let $c$ be a complex-valued coefficient sequence with normalized support of length $N$, and let $\ell_{1}$ and $\ell_{2}$ be different integers between 0 and $N-1$. Then the phase retrieval problem to recover the structured signal in (17.2) from its FOURIER intensity $|\mathcal{F}[f]|$ and the two phases $\arg f\left(N-1-\ell_{1}\right)$ and $\arg f\left(N-1-\ell_{2}\right)$ is almost always uniquely solvable whenever $\ell_{1}+\ell_{2} \neq N-1$. If $\ell_{1}+\ell_{2}=N-1$, then the reconstruction is only unique up to the conjugation and reflection of the coefficient sequence $c$, except for the special case where $\ell_{1}$ and $\ell_{2}$ correspond to the two end points 0 and $N-1$.

Proof. The assertion can be justified similarly to Corollary 17.4. More precisely, using that $\varphi$ is a LAGRANGE function together with (17.1), we obtain the identities $\arg c\left[N-1-\ell_{1}\right]=\arg f\left(N-1-\ell_{1}\right) \quad$ and $\quad \arg c\left[N-1-\ell_{2}\right]=\arg f\left(N-1-\ell_{2}\right)$. 
Since we can recover the FOURIER intensity of the coefficient sequence $c$ from the given intensity $|\mathcal{F}[f]|$ as shown in the proof of Theorem 16.2, a certain instance of the considered problem can be reduced to the discrete-time phase retrieval problem with two additional given phases in the time domain; so the assertion can simply be established by applying the discrete-time analogue in Corollary 11.8.

Remark 17.7. Although Corollary 17.6 contains the special cases $\ell_{1}+\ell_{2}=N-1$, the basic statement is simply that we can recover almost every structured signal $f$ from its FourIER intensity $|\mathcal{F}[f]|$ and the phases arg $f(n)$ for all integers $n$ in the time domain. In other words, using this additional phase information, we can recover almost all structured signals without any ambiguousness.

Finally, we try to enforce uniqueness of the phase retrieval problem for structured signals by exploiting additional interference measurements as considered in Chapter IV. Since the interference measurements are located in the frequency domain, we can drop the additional conditions in the time domain. In other words, the generator function can again be an arbitrary function not equal to zero in $L^{1}$ or $L^{2}$. In the following, we assume the unknown signal $f$ and the reference signal $h$ are structured signals based on the same generator function. This allows us to adapt the main results for a known or unknown reference signal straightforwardly.

Corollary 17.8. Let $f$ and $h$ be two complex-valued structured signals of the form (16.1) based on a complex-valued generator function $\varphi$ in $L^{1}$ or $L^{2}$, where the nonvanishing reference $h$ is known beforehand. Then the structured signal $f$ can be recovered from the FOURIER intensities

$$
|\mathscr{F}[f]| \text { and }|\mathscr{F}[f+h]|
$$

except for one ambiguity at the most.

Proof. Due to the assumption that $f$ and $h$ are based on the same generator function, we can write these signals as

$$
f(t):=\sum_{n \in \mathbb{Z}} c[n] \varphi(t-n) \quad \text { and } \quad h(t):=\sum_{n \in \mathbb{Z}} b[n] \varphi(t-n)
$$

with appropriate coefficient sequences $c$ and $b$. Obviously, the interference $f+h$ is again a structured signal with the same generator function $\varphi$ and the coefficient 
sequence $c+b$. As a direct consequence, we can extract the FOURIER intensity $|\mathscr{F}[c]|$ and also $|\mathscr{F}[c+b]|$ from the given intensities $|\mathscr{F}[f]|$ and $|\mathscr{F}[f+h]|$, and the assertion immediately follows from Theorem 13.4.

Corollary 17.9. Let $f$ and $h$ be two complex-valued structured signals of the form (16.1) based on a conjugate symmetric generator function $\varphi$ in $L^{1}$ or $L^{2}$. If the corresponding zero sets of the coefficient sequences of $f$ and $h$ are disjoint, then both structured signals $f$ and $h$ can be recovered from the FOURIER intensities

$$
|\mathcal{F}[f]|, \quad|\mathscr{F}[h]|, \quad \text { and }|\mathcal{F}[f+h]|
$$

uniquely up to common trivial ambiguities.

Proof. In the same way as in the previous proof, we can reduce the considered phase retrieval problem to the recovery of the coefficient sequences $c$ and $b$ of the structured signals $f$ and $g$ from the discrete-time FourIER intensities $|\mathscr{F}[c]|$, $|\mathscr{F}[b]|$, and $|\mathscr{F}[c+b]|$. Since the corresponding zeros of the sequences $c$ and $b$ are disjoint by assumption, we can make use of Theorem 14.1 and obtain the assertion.

Remark 17.10. Similarly as before, the conjugate symmetry of the generator function ensures that the conjugation and reflection of a structured signal and the signal with the conjugated and reflected coefficient sequence coincide, cf. Remark 17.2. If we abandon this additional assumption, the phase retrieval problem in Corollary 17.9 can possess one further non-trivially different solution. More precisely, this second solution can be obtained by reflecting the coefficient sequences of the structured function $f$ and $h$ of the first solution.

Besides the interference measurements with a known or unknown reference signal, we also consider interferences of the wanted signal with a modulated version of itself. If we generalize this idea to the phase retrieval problem of structured functions (16.1), we assume now that we have access to the FourIER intensity of the signal

$$
f+\mathrm{e}^{\mathrm{i} \alpha} \mathrm{e}^{\mathrm{i} \mu \cdot} f
$$

where $\alpha$ and $\mu$ are two real numbers, cf. Section 15 .

Unfortunately, the additional modulation changes the structure of the signal $f$ such that the considered interference is no longer a structured function of the 
form (16.1) with the original generator function $\varphi$. Moreover, if we look at the FOURIER intensity, which can be written in the form

$$
\left|\mathcal{F}\left[f+\mathrm{e}^{\mathrm{i} \alpha} \mathrm{e}^{\mathrm{i} \mu \cdot} f\right](\omega)\right|=\left|\widehat{\varphi}(\omega) \sum_{n \in \mathbb{Z}} c[n] \mathrm{e}^{-\mathrm{i} \omega n}+\widehat{\varphi}(\omega-\mu) \mathrm{e}^{\mathrm{i} \alpha} \sum_{n \in \mathbb{Z}} c[n] \mathrm{e}^{-\mathrm{i}(\omega-\mu) n}\right|,
$$

we cannot directly determine the required Fourier intensity $\mid \mathcal{F}\left[c+\mathrm{e}^{\mathrm{i} \alpha} \mathrm{e}\right.$ to reduce the problem to a discrete-time version. In spite of this difficulties, we can nevertheless generalize the results about interference measurements of this kind to the new setting by adapting the corresponding proofs appropriately.

Theorem 17.11. Let $f$ be a complex-valued structured signal of the form (16.1) with a generator function in $L^{1}$ or $L^{2}$. If $\mu \neq 2 \pi p / q$ for every $p \in \mathbb{Z}$ and every $q \in\{1, \ldots, N-1\}$, and if the generator function satisfies

$$
\overline{\widehat{\varphi}(\cdot)} \widehat{\varphi}(\cdot-\mu) \not \equiv 0
$$

then the structured signal $f$ can be uniquely recovered up to a rotation from its FOURIER intensity $|\mathcal{F}[f]|$ and the interference measurements

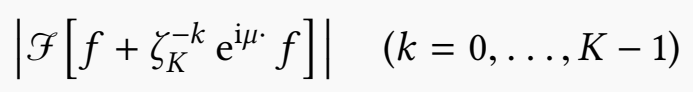

for every integer $K>2$.

Proof. In analogy to the proof of the discrete-time version in Theorem 15.3, we apply the polarization identity (Lemma 15.1) to the given interference measurements

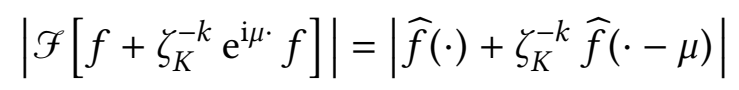

for $k=0, \ldots, K-1$. We then obtain the pointwise identity

$$
\frac{1}{K} \sum_{k=0}^{K-1} \zeta_{K}^{k}\left|\widehat{f}(\omega)+\zeta_{K}^{-k} \widehat{f}(\omega-\mu)\right|^{2}=\overline{\widehat{f}(\omega)} \widehat{f}(\omega-\mu)
$$

for every $\omega$ in the frequency domain. Remembering that the FourIER transform of the structured function $f$ in (16.1) can be written as $\mathscr{F}[f]=\mathscr{F}[\varphi] \mathscr{F}[c]$, we can rearrange the right-hand side and get

$$
\frac{1}{K} \sum_{k=0}^{K-1} \zeta_{K}^{k}\left|\widehat{f}(\omega)+\zeta_{K}^{-k} \widehat{f}(\omega-\mu)\right|^{2}=\overline{\widehat{\varphi}(\omega)} \widehat{\varphi}(\omega-\mu) \overline{\widehat{c}(\omega)} \widehat{c}(\omega-\mu) .
$$

for almost every $\omega$ in the frequency domain. 
Exploiting the additional assumption (17.3), we can now determine

$$
\overline{\widehat{c}(\omega)} \widehat{c}(\omega-\mu)
$$

for almost every $\omega$ in a small interval. Since the Fourier transformed signals $\widehat{c}(\cdot)$ and $\widehat{c}(\cdot-\mu)$ are continuous trigonometric polynomials due to the finite support of the corresponding sequences in the time domain, we can extend (17.4) to the entire frequency domain. Following the lines of the remaining proof of Theorem 15.3, we can use (17.4) to determine the required relative phases and apply Prony's method to recover the coefficient sequence $c$ and hence the structured signal $f$ itself up to rotations.

With an analogous approach as in the discrete-time setting, we can also ensure unique recovery of a structured signal by employing only two different interference measurements. However, in order to determine the required relative phases for the underlying discrete-time problem in the following proof, the generator function of the structured signal have to be conjugate symmetric.

Theorem 17.12. Let $f$ be a structured signal of the form (16.1) with a conjugate symmetric generator function in $L^{1}$ or $L^{2}$. If $\mu \neq 2 \pi p / q$ for every $p \in \mathbb{Z}$ and for every $q \in\{1, \ldots, N-1\}$, and if the generator function satisfies

$$
\overline{\widehat{\varphi}(\cdot)} \widehat{\varphi}(\cdot-\mu) \not \equiv 0,
$$

then the structured signal $f$ can be uniquely recovered up to a rotation from its FOURIER intensity $|\mathcal{F}[f]|$ and the two interference measurements

$$
\left|\mathscr{F}\left[x+\mathrm{e}^{\mathrm{i} \alpha_{1}} \mathrm{e}^{\mathrm{i} \mu \cdot} f\right]\right| \text { and }\left|\mathcal{F}\left[f+\mathrm{e}^{\mathrm{i} \alpha_{2}} \mathrm{e}^{\mathrm{i} \mu \cdot} f\right]\right|
$$

where $\alpha_{1}$ and $\alpha_{2}$ are two real numbers satisfying $\alpha_{1}-\alpha_{2} \neq \pi k$ for all integer $k$.

Proof. As before, the crucial point to transfer our previous observations is the reduction of the considered phase retrieval problem to a discrete-time version. For this purpose, we exemplarily investigate the first interference measurements

$$
\left|\mathcal{F}\left[f+\mathrm{e}^{\mathrm{i} \alpha_{1}} \mathrm{e}^{\mathrm{i} \mu \cdot} f\right]\right|=\left|\widehat{f}(\cdot)+\mathrm{e}^{\mathrm{i} \alpha_{1}} \widehat{f}(\cdot-\mu)\right|
$$

of the structured signal $f$ in (16.1). Extending the squared measurements, we 
obtain the pointwise identity

$$
\begin{aligned}
\mid \widehat{f}(\omega) & +\left.\mathrm{e}^{\mathrm{i} \alpha_{1}} \widehat{f}(\omega-\mu)\right|^{2}-|\widehat{f}(\omega)|^{2}-|\widehat{f}(\omega-\mu)|^{2} \\
= & \mathrm{e}^{-\mathrm{i} \alpha_{1}} \widehat{f}(\omega) \overline{\widehat{f}(\omega-\mu)}+\mathrm{e}^{\mathrm{i} \alpha_{1}} \overline{\widehat{f}(\omega)} \widehat{f}(\omega-\mu) \\
= & \overline{\widehat{\varphi}(\omega)} \widehat{\varphi}(\omega-\mu)\left[\mathrm{e}^{-\mathrm{i} \alpha_{1}} \widehat{c}(\omega) \overline{\widehat{c}(\omega-\mu)}+\mathrm{e}^{\mathrm{i} \alpha_{1}} \overline{\widehat{c}(\omega)} \widehat{c}(\omega-\mu)\right]
\end{aligned}
$$

for every $\omega$ in the frequency domain.

Since we have assumed that the prefactor on the right-hand side is not constantly zero, we can now determine the trigonometric polynomial

$$
\mathrm{e}^{-\mathrm{i} \alpha_{1}} \widehat{c}(\omega) \overline{\widehat{c}(\omega-\mu)}+\mathrm{e}^{\mathrm{i} \alpha_{1}} \overline{\widehat{c}(\omega)} \widehat{c}(\omega-\mu)=2 \mathfrak{R}\left[\mathrm{e}^{\mathrm{i} \alpha_{1}} \overline{\widehat{c}(\omega)} \widehat{c}(\omega-\mu)\right]
$$

for almost every $\omega$ on an appropriate interval and hence for all $\omega$ in the frequency domain. Writing the discrete-time FourIER transform $\widehat{c}$ in its polar representation $|\widehat{c}| \mathrm{e}^{\mathrm{i} \phi}$, where $\phi$ denotes the phase of $\widehat{c}$, and dividing the equation by the occurring moduli, we can thus extract

$$
\mathfrak{R}\left[\mathrm{e}^{\mathrm{i}\left(\phi(\omega-\mu)-\phi(\omega)+\alpha_{1}\right)}\right] \quad \text { and } \quad \mathfrak{R}\left[\mathrm{e}^{\mathrm{i}\left(\phi(\omega-\mu)-\phi(\omega)+\alpha_{2}\right)}\right]
$$

from the given FOURIER intensity and the interference measurements whenever $|\widehat{c}(\omega)|$ and $|\widehat{c}(\omega-\mu)|$ are non-zero. Following the proof of Theorem 15.11, we can now recover the unknown coefficient sequence $c$ and hence the unknown structured function $f$ from these values up to a unimodular constant, which finishes the proof.

Remark 17.13. In some special cases, we can drop the additional assumption that the generator function $\varphi$ have to be conjugate symmetric. For instance, if we consider the two interference measurements

$$
\left|\mathcal{F}\left[f+\mathrm{e}^{\mathrm{i} \mu \cdot} f\right]\right| \text { and }\left|\mathcal{F}\left[f-\mathrm{i} \mathrm{e}^{\mathrm{i} \mu \cdot} f\right]\right|
$$

in analogy to the discrete-time problem in Theorem 15.9, then we can separately determine the real and imaginary part

$$
\mathfrak{R}[\overline{\widehat{f}(\cdot)} \widehat{f}(\cdot-\mu)] \text { and } \mathfrak{J}[\overline{\hat{f}(\cdot)} \widehat{f}(\cdot-\mu)]
$$

with a similar computation as shown in the corresponding proof. Following the lines that lead us to Theorem 17.11, we can now determine the unknown 
coefficient sequence $c$ and hence the wanted structured function $f$ without using the conjugate symmetry of the generator function.

\section{Phase retrieval of arbitrary continuous-time signals}

The considerations of structured functions in the previous two sections have been the first step in order to investigate the continuous-time phase retrieval problem. As we have seen, the phase retrieval problem for structured functions is closely related to the discrete-time problem such that the appearing ambiguities of both problems can be characterized completely analogously. Furthermore, most of the approaches to reduce the set of ambiguities or to enforce uniqueness can be transferred. In this section, we will show that the phase retrieval problem for arbitrary continuous-time signals has a completely different behaviour than the discrete variants. To be more precise, we consider the following continuoustime version of the phase retrieval problem.

Problem 18.1. The continuous-time phase retrieval problem is the problem of recovering a continuous-time signal $f$ in $L^{2}$ with compact support from its FoURIER intensity $|\mathscr{F}[f]|$.

The ambiguousness of this variant of the phase retrieval problem has been studied by Akutowicz [Aku56, Aku57], Walther [Wal63], and Hofstetter [Hof64], for example. In order to deduce similar characterizations like Theorem 5.1 and Theorem 5.5 for the continuous-time setting, we follow the examinations of HofstetTer in [Hof64], where the possible solutions of the phase retrieval problem are presented as an infinite product with respect to the zero sets of the analytic continuations of there FouRIER transforms.

With the intent to determine the analytic continuation of a FourIER transformed signal, we replace the imaginary variable $i \omega$ in the definition of the continuous-time Fourier transform by the complex variable $\zeta$. This means that we consider the (two-sided) LAPLACE transform of a function $f: \mathbb{R} \rightarrow \mathbb{C}$, which is defined by

$$
F(\zeta):=\mathcal{L}[f](\zeta):=\int_{-\infty}^{\infty} f(t) \mathrm{e}^{-\zeta t} \mathrm{~d} t,
$$


see for instance [LeP61, Section 10-2] or [CL72, Section 3.1]. The FourIER transform $\mathscr{F}[f]$ itself now coincides with the two-sided LAPLACE transform on the imaginary axis. More precisely, both transforms are obviously related for a real $\omega$ by the pointwise identity

$$
\widehat{f}(\omega):=\int_{-\infty}^{\infty} f(t) \mathrm{e}^{-\mathrm{i} \omega t} \mathrm{~d} t=F(\mathrm{i} \omega) .
$$

Due to the restriction that the unknown continuous-time signal $f$ in Problem 18.1 has to have a compact support, we can apply the PALEY-WIENER theorem to deduce that the LAPLACE transform $\mathcal{L}[f]$ is an entire function or an integral function of exponential type, see for instance [Boa54, Theorem 6.8.1]. If we instead adapt the PALEY-WIENER theorem to the LAPLACE transform defined above, then the theorem can be stated in the following version.

Theorem 18.2 (PAley-Wiener). The function $F: \mathbb{C} \rightarrow \mathbb{C}$ is an entire function of exponential type and belongs to $L^{2}$ on the imaginary axis if and only if the function $F$ is the LAPLACE transform of a square-integrable function with compact support.

In this context, a function $F: \mathbb{C} \rightarrow \mathbb{C}$ is called entire if the function is analytic over the whole complex plane. If the entire function $F$ moreover grows no faster than an exponential, which means that $F$ can be bounded by

$$
|F(\zeta)| \leq A \mathrm{e}^{B|\zeta|}
$$

then the entire function $F$ is of exponential type, see for instance [You8o, p. 53]. Consequently, together with the identity theorem for holomorphic functions, see for instance [Rem84, Kapitel 8, §1], the theorem of PALEY-WIENER implies that the LAPLACE transform $F$ of a function $f$ with compact support is the unique analytic continuation of the FoURIER transform $\mathscr{F}[f]$ from the imaginary axis to the complex plane because the restriction $F(\mathrm{i} \cdot)$ of the holomorphic LAPLACE transform $F$ to the imaginary axis coincides with the considered FouRIER transform.

To recover the unknown LAPLACE transform $F$ and therewith the signal $f$ itself, we need a suitable representation of the given FourIER intensity. Like in the discrete-time setting, the key instruments are again the autocorrelation signal and the autocorrelation function. For the sake of consistency, we here use a slightly different definition of the continuous-time autocorrelation than HoFSTETTER in [Hof64]. 
Definition 18.3. Let $f$ be a continuous-time signal. The autocorrelation signal $a$ of the signal $f$ is defined by

$$
a(t):=\int_{-\infty}^{\infty} \overline{f(s)} f(s+t) \mathrm{d} s .
$$

The autocorrelation function is the LAPLACE transform $A$ of the autocorrelation signal $a$.

Assuming that the unknown continuous-time signal $f$ has a compact support, we can observe that this property is inherited to the corresponding autocorrelation signal $a$, which also possesses a compact support. Since HöLDER's inequality implies that the autocorrelation signal $a$ can be bounded by a suitable constant almost everywhere, we can furthermore conclude that the autocorrelation signal $a$ also belongs to $L^{2}$. Hence, the autocorrelation function $A$ is always well defined and can be interpreted as the analytic continuation of the FOURIER transform $\widehat{a}$ from the imaginary axis to the complex plane. Similarly to Proposition 3.4, the defined autocorrelation is closely related to the given FourIER intensity.

Proposition 18.4. Let $f$ be a continuous-time signal in $L^{2}$ with compact support. Then the autocorrelation function $A$ is the analytic continuation of the squared FOURIER intensity $|\mathcal{F}[f]|^{2}$ from the imaginary axis to the complex plane.

Proof. Using the definition of the continuous-time autocorrelation in Definition 18.3, we can write the autocorrelation function $A$ of the considered signal $f$ as

$$
A(\zeta)=\int_{-\infty}^{\infty} \int_{-\infty}^{\infty} \overline{f(s)} f(s+t) \mathrm{e}^{-\zeta t} \mathrm{~d} s \mathrm{~d} t
$$

Since the autocorrelation function is well defined, we can now change the order of integration. Shifting the variable of the inner integral, and separating the now independent integrals, we can finally represent the autocorrelation function $f$ in terms of the LAPLACE transform $F$. We obtain the pointwise identity

$$
A(\zeta)=\int_{-\infty}^{\infty} \int_{-\infty}^{\infty} \overline{f(s)} f(t) \mathrm{e}^{-\zeta t} \mathrm{e}^{\zeta s} \mathrm{~d} t \mathrm{~d} s=F(\zeta) \overline{F(-\bar{\zeta})}
$$


If we especially consider the restriction of this identity to the imaginary axis, we have

$$
A(\mathrm{i} \omega)=F(\mathrm{i} \omega) \overline{F(\mathrm{i} \omega)}=\widehat{f}(\omega) \overline{\widehat{f}(\omega)}=|\widehat{f}(\omega)|^{2},
$$

which implies that the restriction of the autocorrelation function $A$ coincides with the squared FourIer intensity $|\mathscr{F}[f]|^{2}$ as claimed. Because the autocorrelation function $A$ is furthermore a holomorphic function by the theorem of PALEy-Wiener, the assertion follows.

After this preliminaries, we are now ready to present the main statement of this section: the characterization of all occurring ambiguities in the continuoustime phase retrieval problem as stated by Hofstetter in [Hof64, Theorem I].

Theorem 18.5 (HOFSTETTER). Let $f$ be a continuous-time signal in $L^{2}$ with compact support. Then the LAPLACE transform of each continuous-time signal $g$ in $L^{2}$ with compact support and the same FOURIER intensity $|\mathcal{F}[g]|=|\mathcal{F}[f]|$ can be written in the form

$$
G(\zeta)=C \zeta^{m} \mathrm{e}^{\zeta \gamma} \prod_{j=1}^{\infty}\left(1-\frac{\zeta}{\eta_{j}}\right) \mathrm{e}^{\frac{\zeta}{\eta_{j}}}
$$

where the absolute value $|C|$ and the imaginary part $\mathfrak{J} \gamma$ of the complex constants $C$ and $\gamma$ coincide for all signals $g$, and where $\eta_{j}$ is chosen for each $j$ from the zero pairs $\left(\xi_{j},-\bar{\xi}_{j}\right)$ of the autocorrelation function $A$.

Astonishingly, the assertion can be easily deduced by applying the HADAMARD factorization theorem, see for instance [Boa54, Theorem 2.7.1] or [Tit39, Theorem 8.24], which allows us to write an entire function of finite order as an infinite product with respect to its zeros. For entire functions of exponential type, we obtain the following special case, see [Hof64, p. 122].

Theorem 18.6 (HADAMARD). If $F$ is an entire function of exponential type with an $m$-fold zero at the origin, then the entire function $F$ can be represented by

$$
F(\zeta)=C \zeta^{m} \mathrm{e}^{\zeta \gamma} \prod_{j=1}^{\infty}\left(1-\frac{\zeta}{\zeta_{j}}\right) \mathrm{e}^{\frac{\zeta}{\xi_{j}}},
$$

with the non-zero zeros $\xi_{j}$ of $F$ and with the complex constants $C$ and $\gamma$, where the possibly infinite product converges absolutely in the whole complex plane. 
Proof of Theorem 18.5. In order to establish the theorem, we follow the lines in the corresponding proof given by Hofstetter in [Hof64, p. 122] and adapt them to the slightly different autocorrelation in Definition 18.3. Since the considered signals $f$ and $g$ have a compact support by assumption, the theorem of PALEY-WIENER yields that the LAPLACE transforms $F$ and $G$ are entire functions of exponential type. Moreover, HADAMARD's factorization theorem now enables us to represent both LAPLACE transforms by

$$
F(\zeta)=C_{1} \zeta^{m_{1}} \mathrm{e}^{\zeta \gamma_{1}} \prod_{j=1}^{\infty}\left(1-\frac{\zeta}{\zeta_{j}}\right) \mathrm{e}^{\frac{\zeta}{\xi_{j}}}
$$

and

$$
G(\zeta)=C_{2} \zeta^{m_{2}} \mathrm{e}^{\zeta \gamma_{2}} \prod_{j=1}^{\infty}\left(1-\frac{\zeta}{\eta_{j}}\right) \mathrm{e}^{\frac{\zeta}{\eta_{j}}}
$$

with respect to their non-zero zeros $\xi_{j}$ and $\eta_{j}$.

Next, considering the second assumption $|\mathscr{F}[g]|=|\mathcal{F}[f]|$, we can conclude that the autocorrelation functions of the continuous-time signals $f$ and $g$ have to coincide since the autocorrelation function is the unique analytic continuation of the squared FourIER intensity, see Proposition 18.4. Using (18.1), we can thus represent the common autocorrelation function $A$ in terms of the LAPLACE transform $F$ or $G$. In this manner, we obtain the identity

$$
A(\zeta)=F(\zeta) \overline{F(-\bar{\zeta})}=G(\zeta) \overline{G(-\bar{\zeta})}
$$

Due to this factorization, all zeros of the autocorrelation function $A$ obviously occur in pairs of the form $\left(\xi_{j},-\bar{\xi}_{j}\right)$, where $\xi_{j}$ is a zero of $F$. Since an analogous observation follows from the factorization with respect to the LAPLACE transform $G$, we can resort the zeros $\eta_{j}$ so that $\eta_{j}=\xi_{j}$ or $\eta_{j}=-\bar{\xi}_{j}$. Further, we can conclude that the multiplicities $m_{1}$ and $m_{2}$ of the zero at the origin must be equal because the above observation is not restricted to the non-zero zeros. Consequently, the possibly infinite products in the factorizations of $A$ coincide, and we can reduce (18.2) to

$$
\left|C_{1}\right|^{2} \mathrm{e}^{\zeta\left(\gamma_{1}-\bar{\gamma}_{1}\right)}=\left|C_{2}\right|^{2} \mathrm{e}^{\zeta\left(\gamma_{2}-\bar{\gamma}_{2}\right)} \quad \text { or } \quad\left|C_{1}\right|^{2} \mathrm{e}^{2 \zeta \mathfrak{I}\left[\gamma_{1}\right]}=\left|C_{2}\right|^{2} \mathrm{e}^{2 \zeta \mathfrak{I}\left[\gamma_{2}\right]}
$$

which shows that the absolute values $\left|C_{1}\right|$ and $\left|C_{2}\right|$ and also the imaginary parts $\mathfrak{J} \gamma_{1}$ and $\mathfrak{J} \gamma_{2}$ coincide.

Similarly to the discrete-time setting, we divide the occurring ambiguities characterized by Theorem 18.5 in two different classes. Since the rotation, the 
time shift by a real number, and the reflection and conjugation of a solution always result in a further solution of the considered phase retrieval problem, cf. Proposition 2.1, these ambiguities are called trivial. In Theorem 5.5, we have been able to show that every non-trivial ambiguity of the discrete-time phase retrieval problem can be represented by an appropriate convolution in the time domain. For the non-trivial ambiguities of the continuous-time problem, we can at least achieve an analogous representation in the frequency domain.

Proposition 18.7. Let $f$ and $g$ be two continuous-time signals in $L^{2}$ with compact support and the same FOURIER intensity $|\mathcal{F}[f]|$. Then there exist two entire functions $F_{1}$ and $F_{2}$ of exponential type such that

$$
F(\zeta)=F_{1}(\zeta) F_{2}(\zeta)
$$

and

$$
G(\zeta)=\mathrm{e}^{\mathrm{i} \alpha} \mathrm{e}^{-\zeta t_{0}} \overline{F_{1}(-\bar{\zeta})} F_{2}(\zeta)
$$

where $\alpha$ and $t_{0}$ are suitable real numbers.

Before we can prove this statement, we need the converse of HADAMARD's factorization theorem, which is also known as BOREL's theorem, see for instance [Mar77, Theorem 10.6]. For this purpose, we define the convergence exponent of a sequence $\left(\xi_{j}\right)_{j \in \mathbb{N}}$ of non-zero complex numbers as the infimum of the positive real numbers $\alpha$ for which the series

$$
\sum_{j=1}^{\infty}\left|\xi_{j}\right|^{-\alpha}
$$

converges, see [Boa54, Definition 2.5.4]. If the sequence $\left(\xi_{j}\right)_{j \in \mathbb{N}}$ does not possess infinitely many components, then the series becomes a finite sum, which implies that the convergence exponent is zero. Adapting Borel's theorem to the special case of entire functions of exponential type, we can state the converse of Theorem 18.6 in the following form.

Theorem 18.8 (BoREL). Let $\left(\xi_{j}\right)_{j \in \mathbb{N}}$ be an infinite sequence of non-zero complex numbers with convergence exponent less than or equal to one. Then the infinite product

$$
F(\zeta)=C \zeta^{m} \mathrm{e}^{\zeta \gamma} \prod_{j=1}^{\infty}\left(1-\frac{\zeta}{\xi_{j}}\right) \mathrm{e}^{\frac{\zeta}{\xi_{j}}}
$$

represents an entire function of exponential type for every complex numbers $C$ and $\gamma$ and every non-negative integer $m$. 
Remark 18.9. Obviously, the statement of BorEL's theorem remains valid if we replace the infinite sequence $\left(\xi_{j}\right)_{j \in \mathbb{N}}$ by a finite one. In other words, we replace the infinite product in Theorem 18.8 by

$$
F(\zeta)=C \zeta^{m} \mathrm{e}^{\zeta \gamma} \prod_{j=1}^{J}\left(1-\frac{\zeta}{\zeta_{j}}\right) \mathrm{e}^{\frac{\zeta}{\xi_{j}}}
$$

for a non-negative integer $J$.

Proof of Proposition 18.7. Applying Hofstetter's characterization in Theorem 18.5, we can represent the LAPLACE transforms $F$ and $G$ by

$$
F(\zeta)=C_{1} \zeta^{m} \mathrm{e}^{\zeta \gamma_{1}} \prod_{j=1}^{\infty}\left(1-\frac{\zeta}{\xi_{j}}\right) \mathrm{e}^{\frac{\zeta}{\xi_{j}}}
$$

and

$$
G(\zeta)=C_{2} \zeta^{m} \mathrm{e}^{\zeta \gamma_{2}} \prod_{j=1}^{\infty}\left(1-\frac{\zeta}{\eta_{j}}\right) \mathrm{e}^{\frac{\zeta}{\eta_{j}}}
$$

with $\left|C_{1}\right|=\left|C_{2}\right|, \mathfrak{J} \gamma_{1}=\mathfrak{J} \gamma_{2}$, and $\eta_{j} \in\left(\xi_{j},-\bar{\xi}_{j}\right)$ due to the assumption that the FoURIER intensities $|\mathscr{F}[f]|$ and $|\mathscr{F}[g]|$ coincide. In analogy to the modified zero sets in the discrete-time setting, we can here resort the zeros of $G$ such that

$$
\eta_{j}=\left\{\begin{array}{cl}
-\bar{\xi}_{j} & \xi_{j} \in \Lambda \\
\xi_{j} & \text { else }
\end{array}\right.
$$

for an appropriate subset $\Lambda$ of the corresponding zero set $\Xi:=\left\{\xi_{j}: j \in \mathbb{N}\right\}$ of $f$.

Based on this subset, we define the two possibly infinite products $F_{1}$ and $F_{2}$ by

$$
F_{1}(\zeta)=\prod_{\xi_{j} \in \Lambda}\left(1-\frac{\zeta}{\xi_{j}}\right) \mathrm{e}^{\frac{\zeta}{\xi_{j}}} \quad \text { and } \quad F_{2}(\zeta)=C_{1} \zeta^{m} \mathrm{e}^{\zeta \gamma_{1}} \prod_{\xi_{j} \in \Xi \backslash \Lambda}\left(1-\frac{\zeta}{\xi_{j}}\right) \mathrm{e}^{\frac{\zeta}{\xi_{j}}}
$$

Due to the fact that the convergence exponent of the zeros of an entire function is always less than or equal to the order of the entire function, see for instance [Boa54, Theorem 2.5.18], the zeros $\xi_{j}$ of the LAPLACE transform $F$ can at most have the convergence exponent one. Since the zeros of $F_{1}$ and $F_{2}$ are merely subsets of the zeros of $F$, the corresponding convergence exponents thus have to be less than or equal to one too. Borel's theorem now implies that $F_{1}$ and $F_{2}$ are entire functions of exponential type. 
By the construction of the entire functions $F_{1}$ and $F_{2}$, we obviously have the factorization $F=F_{1} F_{2}$. In order to achieve the factorization of $G$, we consider the reflection of the first factor given by

$$
\overline{F_{1}(-\bar{\zeta})}=\prod_{\xi_{j} \in \Lambda}\left(1-\frac{\zeta}{-\bar{\xi}_{j}}\right) \mathrm{e}^{\frac{\zeta}{-\bar{\xi}_{j}}}
$$

Hence, the reflection $\overline{F_{1}\left(-^{-}\right)}$obviously possesses the zeros $\eta_{j}=-\bar{\xi}_{j}$ for all $\xi_{j}$ in $\Lambda$, which implies that the zeros of the product $\overline{F_{1}\left(-^{-}\right)} F_{2}$ and the LAPLACE transform $G$ coincide.

Finally, since the absolute values $\left|C_{1}\right|$ and $\left|C_{2}\right|$ and the imaginary parts $\mathfrak{J} \gamma_{1}$ and $\mathfrak{J} \gamma_{2}$ have to be equal by the characterization in Theorem 18.5, the entire functions $\overline{F_{1}\left(-^{-}\right)} F_{2}$ and $G$ can only differ by a rotation $\mathrm{e}^{\mathrm{i} \alpha}$ and a time shift $\mathrm{e}^{-\zeta t_{0}}$. Choosing the real numbers $\alpha$ and $t_{0}$ suitably, we obtain the wanted factorization in the assertion.

Remark 18.10. Although the ambiguities of the phase retrieval problem to recover continuous-time signals can be represented in the frequency domain similarly to Theorem 5.5, we unfortunately cannot retransform the found factorization to describe the ambiguities by a convolution of two continuous-time signals in the time domain. Here the main problem is that we cannot ensure the square integrability of the factors $F_{1}$ and $F_{2}$ along the imaginary axis. For example, if the subset $\Lambda$ only contains a finite number of zeros, then the factor $F_{1}$ is obviously the product of a polynomial and an exponential, whose restriction to the imaginary axis cannot belong to $L_{2}$. Consequently, the entire functions $F_{1}$ and $F_{2}$ do not have to be the LAPLACE transform of a square-integrable continuous-time signal with compact support as stated by the theorem of PALEY-WIEnER (Theorem 18.2). Even if we use the theory of distribution, we cannot ensure that the factors $F_{1}$ and $F_{2}$ are the LAPLACE transform of two distribution with compact support.

Remark 18.11. Applying PALEY-Wiener's theorem, we can interpret Proposition 18.7 in a slightly different way: if the restrictions of the entire functions $F$ and $G$ of exponential type to the imaginary axis coincide and belong to $L^{2}$, we can always find two entire functions $F_{1}$ and $F_{2}$ to factorize $F$ and $G$ in the manner of Proposition 18.7. This observation can now be generalized to entire function of arbitrary order, where the additional assumption of the square-integrable restriction can be revoked, see [Mar14, Lemma 1]. 


\section{The relation between the discrete-time and continuous- time problem}

Comparing the characterization of the arising ambiguities in the discrete-time and continuous-time phase retrieval, we observe that both problems have a quite different behaviour. The discrite-time phase retrieval problem possesses only a finite number of non-trivial solutions, whereas the continuous-time counterpart usually has infinitely many. In this section, we now study the issue: how are the discrete-time and continuous-time versions of one and the same phase retrieval problem related?

For this purpose, we revert to the phase retrieval problem of structured functions. Looking back at Theorem 16.2, we notice that the solutions can be represented similarly to the characterization in Theorem 5.1 for the discrete-time setting. The main reason for this behaviour is that the phase retrieval problem to recover a certain structured function can be completely reduced to the discrete-time phase retrieval problem to recover the coefficient sequence.

Nevertheless, a structured function is a continuous-time signal, and if the generator function is contained in $L^{2}$ and possesses a compact support, then the resulting structured functions are square-integrable functions with compact support too. Consequently, the ambiguities of the continuous-time phase retrieval problem to recover such a structured function can be characterized additionally by Theorem 18.5 .

All in all, the phase retrieval problem to recover a structured function unifies the characteristics of the discrete-time and continuous-time setting. To compare the characterization of all possible solutions in Theorem 5.1 and Theorem 18.5, we firstly determine the zero set of the LAPLACE transform of a structured function.

Proposition 19.1. Let $f$ be a structured function of the form (16.1) whose generator function $\varphi$ is contained in $L^{2}$ and possesses a compact support. Then the zeros of the LAPLACE transform $F$ are given by the union

$$
\left\{\eta_{j}: j \in \mathbb{N}\right\} \cup\left\{-\ln \left|\beta_{j}\right|-\mathrm{i} \arg \beta_{j}+2 \pi \mathrm{i} \ell: j=1, \ldots, N-1, \ell \in \mathbb{Z}\right\},
$$

where $\eta_{j}$ denotes the zeros of the LAPLACE transform $\Phi$ of $\varphi$ and $\beta_{j}$ the corresponding zeros of the coefficients sequence $c$ with support length $N$.

Proof. Using the properties of the LAPLACE transform, we can represent the structured function (16.1) in the frequency domain by

$$
F(\zeta)=\mathcal{L}\left[\sum_{n \in \mathbb{Z}} c[n] \varphi(\cdot-n)\right](\zeta)=\Phi(\zeta) \sum_{n \in \mathbb{Z}} c[n] \mathrm{e}^{-\zeta n}
$$


where the sum on the right-hand side is the discrete-time LAPLACE transform of the discrete-time signal $c$. In complete analogy to the Fourier transform of a discrete-time signal with finite support, we can now write the corresponding LAPLACE transform of $c$ as an algebraic polynomial in $\mathrm{e}^{-\zeta}$. More precisely, assuming that the support of the coefficient sequence $c$ is given by $\left\{n_{0}, \ldots, n_{0}+N-1\right\}$, we renumber the coefficients by $c\left[k+n_{0}\right] \rightarrow c[k]$. In this manner, the LAPLACE transform $F$ becomes

$$
F(\zeta)=\mathrm{e}^{-\zeta n_{0}} \Phi(\zeta) \sum_{n=0}^{N-1} c[n] \mathrm{e}^{-\zeta n}=\mathrm{e}^{-\zeta n_{0}} \Phi(\zeta) c[N-1] \prod_{j=1}^{N-1}\left(\mathrm{e}^{-\zeta}-\beta_{j}\right)
$$

where we have factorized the occurring polynomial with respect to its zeros $\beta_{j}$.

Consequently, the LAPLACE transform $F$ is zero if and only if the LAPLACE transform $\Phi$ of the generator function is zero or the exponential $\mathrm{e}^{-\zeta}$ coincides with at least one of the corresponding zeros $\beta_{j}$. Since the values $\zeta$ that fulfil the second condition are given by

$$
-\ln \left|\beta_{j}\right|-\mathrm{i} \arg \beta_{j}+2 \pi \mathrm{i} \ell
$$

for every integer $\ell$, the assertion follows.

If we consider the relation between the corresponding zeros $\beta_{j}$ of the coefficient sequence and the zeros of the LAPLACE transform $\xi_{j}$ in (19.1), we can observe that each zero $\beta_{j}$ corresponds to an infinite set of zeros $\xi_{j}$ lying on the line $-\ln \left|\beta_{j}\right|+\mathrm{i} \mathbb{R}$. Since the non-trivial ambiguities in the phase retrieval of a structured function $f$ can be constructed by reflecting the corresponding zeros $\beta_{j}$ of the coefficient sequence on the unit circle, we investigate the influence of such a reflection to the zeros of the LAPLACE transform $F$.

Proposition 19.2. The reflection of a corresponding zero $\beta_{j}$ of a structured function $f$ results in the reflection of the related zeros (19.1) of the LAPLACE transform $F$ on the imaginary axis.

Proof. As discussed in the proof of Proposition 19.1, each corresponding zero $\beta_{j}$ is related to the zeros (19.1) of the LAPLACE transform $F$. If we now replace the zero $\beta_{j}$ by its reflection at the unit circle, then the values $\zeta$ that satisfy the equation

$$
\mathrm{e}^{-\zeta}=\bar{\beta}_{j}^{-1}
$$


are obviously given by

$$
-\ln \left|\bar{\beta}_{j}^{-1}\right|-\mathrm{i} \arg \bar{\beta}_{j}^{-1}+2 \pi \mathrm{i} \ell=\ln \left|\beta_{j}\right|-\mathrm{i} \arg \beta_{j}+2 \pi \mathrm{i} \ell
$$

for every integer $\ell$. In other words, we reflect all related zeros (19.1) at the imaginary axis as claimed.

In summery, the phase retrieval problem for structured functions interrelates the discrete-time and continuous-time versions of the phase retrieval problem. As seen in Proposition 19.1, each zero $\beta_{j}$ of the underlying discrete-time coefficient sequence is related to infinitely many corresponding zeros $\xi_{j}$ of the actual continuous-time signal on the line $-\ln \left|\beta_{j}\right|+\mathrm{i} \mathbb{R}$, while the reflected zero $\bar{\beta}_{j}^{-1}$ is related to the parallel line $\ln \left|\beta_{j}\right|+\mathrm{i} \mathbb{R}$.

Theoretically, the reflection of an arbitrary set of corresponding zeros $\xi_{j}$ at the imaginary axis now yields a further solution of the continuous-time phase retrieval problem, see Theorem 18.5. However, this solution usually violates the assumed structure in (16.1). In order to preserve the structure of the function, we have to reflect all zeros $\xi_{j}$ related to a subset of the corresponding zeros $\beta_{j}$ at the imaginary axis, see Proposition 19.2. Thus, the additional structure of the function in (16.1), or the additional structure of a discrete-time signal, always reduces the usually infinite set of non-trivial ambiguities in the continuous-time setting to a finite set.

Although the phase retrieval problem for structured function can be interpreted as a special case of the continuous-time problem for generator functions in $L^{2}$ with compact support, the original definition of a structured function in (16.1) is not restricted to those generators. In this manner, the assumed structure of the unknown continuous-time signal allows us to characterize the ambiguities appearing in the continuous-time phase retrieval problem also for signals without a finite support, which is not covered by the characterization in Theorem 18.5 .

\section{Ensuring uniqueness in the continuous-time phase re- trieval}

Differently from the discrete-time phase retrieval problem and the phase retrieval problem for structured functions, the continuous-time version to recover a certain signal usually possesses infinitely many non-trivial ambiguities. Hence, 
we are once more faced with the question: how can we ensure the unique recovery of the unknown signal, or how can we at least reduce the occurring ambiguities to a sufficiently small set.

Unfortunately, the lack of an explicit representation of the appearing ambiguities in the time domain in analogy to Theorem 5.1 or Theorem 5.5 prevents a direct generalization of our findings in Chapter II and III, where we have investigated the additional non-negativity of the wanted signal and further given moduli or phases of the signal values in the time domain. With a completely different approach and suitable assumptions, KLiBAnov, SACKs, and TikHonravov show that additional information about the unknown signal in the time domain can still be exploited to achieve the uniqueness of the continuous-time problem, see [KST95]; so a continuous-time signal $f:(0, \infty) \rightarrow \mathbb{C}$ is uniquely determined by its Fourier intensity $|\mathcal{F}[f]|$ and the initial segment $\left.f\right|_{(0, \varepsilon)}$ for any $\varepsilon>0$.

In order to overcome the difficulties in the time domain, we restrict our endeavours to achieve the desired uniqueness of the continuous-time phase retrieval problem to the generalization of our findings in Chapter IV. In other words, we try to enforce the uniqueness of Problem 18.1 by employing different kinds of interference measurements. Once more, we start our investigations by considering the interference of the unknown signal with a known reference.

Similarly to the discrete-time version in Theorem 13.4, KLIBANOv et al. show that the additional interference measurement with a known reference can nearly ensure the unique recovery of a distribution with compact support, see [KST95, Proposition 6.5]. Adapting the proposition of KLIBANov et al. to the phase retrieval problem of continuous-time signals in $L^{2}$ with compact support, we have the following statement.

Proposition 20.1 (KLIBanov et al.). Let $f$ and $h$ be two continuous-time signals in $L^{2}$ with finite support, where the non-vanishing reference signal is known beforehand. Then the signal $f$ can be recovered from the FOURIER intensities

$$
|\mathcal{F}[f]| \text { and }|\mathcal{F}[f+h]|
$$

except for at most one ambiguity.

Proof. Adapting our findings in Section 13.2, we here give a proof that differs from the argumentation given by KLIBANOv et al. Writing the Fourier transforms of the signals $f$ and $h$ in their polar representations

$$
\mathscr{F}[f]=|\mathcal{F}[f]| \mathrm{e}^{\mathrm{i} \phi} \quad \text { and } \quad \mathscr{F}[h]=|\mathcal{F}[h]| \mathrm{e}^{\mathrm{i} \psi},
$$


where $\phi$ and $\psi$ denote the corresponding phase functions, we can again determine the phase difference $\phi-\psi$ for every $\omega$ in the frequency domain by

$$
\phi(\omega)-\psi(\omega)= \pm \arccos \left(\frac{|\mathcal{F}[f+h](\omega)|^{2}-|\mathscr{F}[f](\omega)|^{2}-|\mathscr{F}[h](\omega)|^{2}}{2|\mathscr{F}[f](\omega)||\mathcal{F}[h](\omega)|}\right)+2 \pi k
$$

with an appropriate integer $k$ whenever $\mathscr{F}[f](\omega)$ and $\mathscr{F}[h](\omega)$ are non-zero.

Due to the fact that the FourIER transforms of $f$ and $h$ are no longer trigonometric polynomials, we here need different arguments to conclude that at most two distinct phase differences can occur. As restriction of an entire function, the FourIER transforms $\mathscr{F}[f]$ and $\mathscr{F}[h]$ are continuous, which implies that we can find a small interval where the sign of the phase difference $\phi-\psi$ in (20.1) is everywhere plus or minus. Further, since $\phi-\psi$ is obviously the phase function of the product $\mathscr{F}[f] \overline{\mathscr{F}[h]}$, which is the restriction of the entire function $F(\cdot) \overline{H\left(-^{-}\right)}$to the imaginary axis, we can extend $\phi-\psi$ uniquely from the interval to the complete frequency domain. Consequently, there exist at most two distinct phase differences $\phi-\psi$.

Following the proof of Theorem 13.4, the FOURIER transform of the possible second solution of the considered phase retrieval problem has to be of the form

$$
\mathscr{F}[\breve{f}]=|\mathcal{F}[f]| \mathrm{e}^{-\mathrm{i} \phi+2 \mathrm{i} \psi},
$$

which completes the proof.

Remark 20.2. The main benefit of the proof of Proposition 20.1 given above is that we obtain an explicit representation of the second possible solution. Considering the FOURIER transform (20.2), we can have doubts whether the corresponding continuous-time signal is a really signal with compact support and hence a valid solution of the problem.

Indeed, the FOURIER transform (20.2) does not have to be the restriction of an entire function or even a continuous function at all because the phase $\psi$ of the continuous function $\mathcal{F}[h]$ itself can possesses discontinuities. Here the theorem of PALEY-Wiener implies that the second solution $\breve{f}$ does not have a compact support and is thus an invalid solution of the considered continuous-time phase retrieval problem.

Next, we replace the known reference signal $h$ within the interference $f+h$ by an unknown reference. Based on the proof of Theorem 14.1, we can now show that the continuous-time signal $f$ together with the unknown reference $h$ are uniquely determined by the Fourier intensities of $f, h$, and $f+h$ up to com- 
mon trivial ambiguities. This means that we can recover $f$ and $h$ up to common rotations or time shifts or up to the reflection and conjugation of both signals.

Theorem 20.3. Let $f$ and $h$ be two continuous-time signals in $L^{2}$ with compact support. If the non-zero zeros of the LAPLACE transformed signal $F$ and $H$ form disjoint sets, then both signals $f$ and $h$ can be recovered from the FOURIER intensities

$$
|\mathscr{F}[f]|, \quad|\mathscr{F}[h]|, \quad \text { and, }|\mathcal{F}[f+h]|
$$

uniquely up to common trivial ambiguities.

Proof. Let $\breve{f}$ and $\breve{h}$ be a further solution pair of the considered problem with

$$
|\mathscr{F}[f]|=|\mathscr{F}[\breve{f}]|, \quad|\mathscr{F}[h]|=|\mathscr{F}[\breve{h}]|, \quad \text { and } \quad|\mathscr{F}[f+h]|=|\mathscr{F}[\breve{f}+\breve{h}]| .
$$

Applying Proposition 18.7, we can represent the two solution pairs in the frequency domain by an appropriate factorization of the LAPLACE transform $F$ and $H$ of the original signals. In this manner, we obtain the factorizations

$$
F(\zeta)=F_{1}(\zeta) F_{2}(\zeta) \quad \text { and } \quad \breve{F}(\zeta)=\mathrm{e}^{\mathrm{i} \alpha_{1}} \mathrm{e}^{-\zeta t_{1}} \overline{F_{1}(-\bar{\zeta})} F_{2}(\zeta)
$$

and further

$$
H(\zeta)=H_{1}(\zeta) H_{2}(\zeta) \text { and } \quad \breve{H}(\zeta)=\mathrm{e}^{\mathrm{i} \alpha_{2}} \mathrm{e}^{-\zeta t_{2}} \overline{H_{1}(-\bar{\zeta})} H_{2}(\zeta)
$$

for some real numbers $\alpha_{1}, \alpha_{2}, t_{1}, t_{2}$ and entire functions $F_{1}, F_{2}, H_{1}, H_{2}$.

In the next step, we consider the analytic continuation of the squared interference measurement or the corresponding autocorrelation function, see Proposition 18.4. With the representation in (18.1), we can now write the given interference measurement as

$$
(F(\zeta)+H(\zeta))(\overline{F(-\bar{\zeta})}+\overline{H(-\bar{\zeta})})=(\breve{F}(\zeta)+\breve{H}(\zeta))(\overline{\breve{F}(-\bar{\zeta})}+\overline{\breve{H}(-\bar{\zeta})})
$$

or in the simplified form

$$
F(\zeta) \overline{H(-\bar{\zeta})}+\overline{F(-\bar{\zeta})} H(\zeta)=\breve{F}(\zeta) \overline{\breve{H}(-\bar{\zeta})}+\overline{\breve{F}(-\bar{\zeta})} \breve{H}(\zeta)
$$

Incorporating the found factorizations of $F$ and $H$, we obtain

$$
F_{1}(\zeta) F_{2}(\zeta) \overline{H_{1}(-\bar{\zeta})} \overline{H_{2}(-\bar{\zeta})}+\overline{F_{1}(-\bar{\zeta})} \overline{F_{2}(-\bar{\zeta})} H_{1}(\zeta) H_{2}(\zeta)
$$




$$
\begin{aligned}
= & \mathrm{e}^{\mathrm{i}\left(\alpha_{1}-\alpha_{2}\right)} \mathrm{e}^{-\zeta\left(t_{1}-t_{2}\right)} \overline{F_{1}(-\bar{\zeta})} F_{2}(\zeta) H_{1}(\zeta) \overline{H_{2}(-\bar{\zeta})} \\
& +\mathrm{e}^{\mathrm{i}\left(\alpha_{2}-\alpha_{1}\right)} \mathrm{e}^{-\zeta\left(t_{2}-t_{1}\right)} F_{1}(\zeta) \overline{F_{2}(-\bar{\zeta})} \overline{H_{1}(-\bar{\zeta})} H_{2}(\zeta)
\end{aligned}
$$

and thus

$$
\begin{aligned}
& {\left[\mathrm{e}^{-\mathrm{i} \alpha_{1}} \mathrm{e}^{\zeta t_{1}} F_{1}(\zeta) \overline{H_{1}(-\bar{\zeta})}-\mathrm{e}^{-\mathrm{i} \alpha_{2}} \mathrm{e}^{\zeta t_{2}} \overline{F_{1}(-\bar{\zeta})} H_{1}(\zeta)\right]} \\
& \cdot\left[\mathrm{e}^{\mathrm{i} \alpha_{1}} \mathrm{e}^{-\zeta t_{1}} F_{2}(\zeta) \overline{H_{2}(-\bar{\zeta})}-\mathrm{e}^{\mathrm{i} \alpha_{2}} \mathrm{e}^{-\zeta t_{2}} \overline{F_{2}(-\bar{\zeta})} H_{2}(\zeta)\right]=0 .
\end{aligned}
$$

Remembering that $F_{1}, F_{2}, H_{1}$, and $H_{2}$ are entire functions, we observe that both factors in (20.3) are entire functions too, and that at least one of both factors thus has to be constantly zero. In order to investigate the two different cases more precisely, we look back at the explicit construction of the entire functions $F_{1}$ and $F_{2}$ in the proof of Proposition 18.7. Using a similar procedure for $H_{1}$ and $H_{2}$, and denoting the sets of all non-zero zeros of $F$ and $H$ by $\Xi_{1}$ and $\Xi_{2}$ respectively, we can represent the four functions by

$$
F_{1}(\zeta)=\prod_{\xi_{j} \in \Lambda_{1}}\left(1-\frac{\zeta}{\xi_{j}}\right) \mathrm{e}^{\frac{\zeta}{\xi_{j}}} \quad \text { and } \quad F_{2}(\zeta)=C_{1} \zeta^{m_{1}} \mathrm{e}^{\zeta \gamma_{1}} \prod_{\xi_{j} \in \Xi_{1} \backslash \Lambda_{1}}\left(1-\frac{\zeta}{\xi_{j}}\right) \mathrm{e}^{\frac{\zeta}{\xi_{j}}}
$$

and further

$$
H_{1}(\zeta)=\prod_{\eta_{j} \in \Lambda_{2}}\left(1-\frac{\zeta}{\eta_{j}}\right) \mathrm{e}^{\frac{\zeta}{\eta_{j}}} \text { and } H_{2}(\zeta)=C_{2} \zeta^{m_{2}} \mathrm{e}^{\zeta \gamma_{2}} \prod_{\eta_{j} \in \Xi_{2} \backslash \Lambda_{2}}\left(1-\frac{\zeta}{\eta_{j}}\right) \mathrm{e}^{\frac{\zeta}{\eta_{j}}},
$$

where $\Lambda_{1}$ and $\Lambda_{2}$ are appropriate subsets of $\Xi_{1}$ and $\Xi_{2}$.

In the following, we firstly assume that the second factor of (20.3) is zero, which directly implies that the equation

$$
\begin{gathered}
(-1)^{m_{2}} C_{1} \bar{C}_{2} \mathrm{e}^{\mathrm{i} \alpha_{1}} \zeta^{m_{1}+m_{2}} \mathrm{e}^{-\zeta\left(t_{1}-\gamma_{1}+\bar{\gamma}_{2}\right)} \prod_{\xi_{j} \in \Xi_{1} \backslash \Lambda_{1}}\left(1-\frac{\zeta}{\zeta_{j}}\right) \mathrm{e}^{\frac{\zeta}{\xi_{j}}} \prod_{\eta_{j} \in \Xi_{2} \backslash \Lambda_{2}}\left(1-\frac{\zeta}{-\bar{\eta}_{j}}\right) \mathrm{e}^{\frac{\zeta}{-\bar{\eta}_{j}}} \\
=(-1)^{m_{1}} \bar{C}_{1} C_{2} \mathrm{e}^{\mathrm{i} \alpha_{2}} \zeta^{m_{1}+m_{2}} \mathrm{e}^{-\zeta\left(t_{2}+\bar{\gamma}_{1}-\gamma_{2}\right)} \prod_{\xi_{j} \in \Xi_{1} \backslash \Lambda_{1}}\left(1-\frac{\zeta}{-\bar{\xi}_{j}}\right) \mathrm{e}^{\frac{\zeta}{-\bar{\xi}_{j}}} \prod_{\eta_{j} \in \Xi_{2} \backslash \Lambda_{2}}\left(1-\frac{\zeta}{\eta_{j}}\right) \mathrm{e}^{\frac{\zeta}{\eta_{j}}}
\end{gathered}
$$

holds for every $\zeta$ in the complex plane. Since the possibly infinite products above are again entire functions by construction or by BOREL's theorem (Theorem 18.8), the zeros on both sides of the equality have to coincide. However, due to the assumption that the zeros $\xi_{j}$ and $\eta_{j}$ of the LAPLACE transforms $F$ and $H$ are pairwise 
distinct, the zero sets $\Xi_{1} \backslash \Lambda_{1}$ and $\Xi_{2} \backslash \Lambda_{2}$ of $F_{2}$ and $H_{2}$ have to be invariant under reflection at the imaginary axis.

Based on this observation, we can immediately conclude that the entire functions $F_{2}$ and $H_{2}$ are invariant under reflection and conjugation up to an additional rotation and modulation. More precisely, we obtain the identities

$$
\begin{aligned}
\overline{F_{2}(-\bar{\zeta})} & =(-1)^{m_{1}} \bar{C}_{1} \zeta^{m_{1}} \mathrm{e}^{-\zeta \bar{\gamma}_{1}} \prod_{\xi_{j} \in \Xi_{1} \backslash \Lambda_{1}}\left(1-\frac{\xi}{-\bar{\zeta}_{j}}\right) \mathrm{e}^{\frac{\zeta}{\bar{\zeta}_{j}}} \\
& =(-1)^{m_{1}} \mathrm{e}^{-2 \mathrm{i} \arg C_{1}} \mathrm{e}^{-2 \zeta \Re\left[\gamma_{1}\right]} F_{2}(\zeta)
\end{aligned}
$$

and similarly

$$
\overline{H_{2}(-\bar{\zeta})}=(-1)^{m_{2}} \mathrm{e}^{-2 \mathrm{i} \arg C_{2}} \mathrm{e}^{-2 \zeta \Re\left[\gamma_{2}\right]} H_{2}(\zeta)
$$

Incorporating these identities in the representation of $\breve{F}$ and $\breve{H}$, we can describe the second solution pair in the frequency domain by

$$
\breve{F}(\zeta)=(-1)^{m_{1}} \mathrm{e}^{\mathrm{i}\left(\alpha_{1}+2 \arg C_{1}\right)} \mathrm{e}^{-\zeta\left(t_{1}-2 \mathfrak{R}\left[\gamma_{1}\right]\right)} \overline{F(-\bar{\zeta})}
$$

and

$$
\breve{H}(\zeta)=(-1)^{m_{2}} \mathrm{e}^{\mathrm{i}\left(\alpha_{2}+2 \arg C_{2}\right)} \mathrm{e}^{-\zeta\left(t_{2}-2 \mathfrak{R}\left[\gamma_{2}\right]\right)} \overline{H(-\bar{\zeta})}
$$

Hence, the continuous-time signals $\breve{f}$ and $\breve{h}$ are merely rotations and shifts of the original signals $f$ and $h$.

It remains to prove that the occurring rotations and shifts coincide. For this purpose, we revisit equation (20.4). Considering that the zeros and hence the possibly infinite products on both sides are equal, we can reduce (20.4) to

$$
(-1)^{m_{2}} C_{1} \bar{C}_{2} \mathrm{e}^{\mathrm{i} \alpha_{1}} \mathrm{e}^{-\zeta\left(t_{1}-\gamma_{1}+\bar{\gamma}_{2}\right)}=(-1)^{m_{1}} \bar{C}_{1} C_{2} \mathrm{e}^{\mathrm{i} \alpha_{2}} \mathrm{e}^{-\zeta\left(t_{2}+\bar{\gamma}_{1}-\gamma_{2}\right)}
$$

or, by rearranging and combining the individual factors, to

$$
(-1)^{m_{1}} \mathrm{e}^{\mathrm{i} \alpha_{1}+2 \arg C_{1}} \mathrm{e}^{-\zeta\left(t_{1}-2 \mathfrak{R}\left[\gamma_{1}\right]\right)}=(-1)^{m_{2}} \mathrm{e}^{\mathrm{i} \alpha_{2}+2 \arg C_{2}} \mathrm{e}^{-\zeta\left(t_{2}-2 \mathfrak{R}\left[\gamma_{2}\right]\right)},
$$

which verifies our conjecture that the second solution pair $\breve{f}$ and $\breve{h}$ coincides with first solution pair $f$ and $h$ up to common trivial ambiguities.

For the second case, where the first factor of (20.3) is constantly zero, an analogous and slightly simpler argumentation yields the representations

$$
\breve{F}(\zeta)=\mathrm{e}^{\mathrm{i} \alpha_{1}} \mathrm{e}^{-\zeta t_{1}} F(\zeta) \text { and } \breve{H}(\zeta)=\mathrm{e}^{\mathrm{i} \alpha_{2}} \mathrm{e}^{-\zeta t_{2}} H(\zeta),
$$


where the occurring rotations and time shifts again coincide. In other words, since we have $\mathrm{e}^{\mathrm{i} \alpha_{1}} \mathrm{e}^{-\zeta t_{1}}=\mathrm{e}^{\mathrm{i} \alpha_{2}} \mathrm{e}^{-\zeta t_{2}}$, the signals $\breve{f}$ and $\breve{h}$ of the second solution pair are trivial ambiguities of the original signals $f$ and $h$ caused be the same rotation and shift.

The last approach to achieve the uniqueness of the continuous-time phase retrieval problem considered in this section is again the idea of using interference measurements of the unknown signal with a modulated version of the signal itself. Generalizing the main results of Section 15, we will establish two different theorems, which show that each continuous-time signal in $L_{2}$ with compact support can be uniquely recovered from an appropriate set of interference measurements.

Theorem 20.4. Let $f$ be a continuous-time signal in $L^{2}$ with compact support. Then the signal $f$ can be uniquely recovered up to a rotation from its FOURIER intensity $|\mathcal{F}[f]|$ and the interference measurements

$$
\mid \mathcal{F}\left[f+\zeta_{K}^{-k} \mathrm{e}^{\mathrm{i} \mu \cdot f] \mid}(k=0, \ldots, K-1 ; \mu \in M)\right.
$$

for every integer $K$ greater than two and every open neighbourhood $M$ around zero.

Proof. Due to the assumption that the unknown signal $f$ is a square-integrable function with compact support, the theorem of PALEY-WIENER (Theorem 18.2) implies that the FoURIER transform $\mathscr{F}[f]$ is the restriction of an entire function and thus has to be continuous. Consequently, if the signal $f$ does not vanish everywhere, we can find a point $\omega_{0}$ together with an open neighbourhood where the Fourier transform $\mathscr{F}[f]$ is non-zero.

Similarly to the discrete-time version in Theorem 15.3, the key element of the proof is now to exploit the additional interference measurements

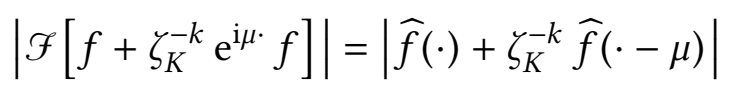

by using the polarization identity in Lemma 15.1. In this manner, we obtain the pointwise identity

$$
\frac{1}{K} \sum_{k=0}^{K-1} \zeta_{K}^{k}\left|\widehat{f}\left(\omega_{0}\right)+\zeta_{K}^{-k} \widehat{f}\left(\omega_{0}-\mu\right)\right|^{2}=\overline{\widehat{f}\left(\omega_{0}\right)} \widehat{f}\left(\omega_{0}-\mu\right)
$$


for every $\mu$ in the open set $M$. Writing the FourIER transform $\mathscr{F}[f]$ in its polar representation $|\mathcal{F}[f]| \mathrm{e}^{\mathrm{i} \phi}$, where $\phi$ denotes the corresponding phase function, we can now extract the relative phases $\phi\left(\omega_{0}-\mu\right)-\phi\left(\omega_{0}\right)$ from

$$
\frac{1}{K} \sum_{k=0}^{K-1} \zeta_{K}^{k}\left|\widehat{f}\left(\omega_{0}\right)+\zeta_{K}^{-k} \widehat{f}\left(\omega_{0}-\mu\right)\right|^{2}=\left|\widehat{f}\left(\omega_{0}\right)\right|\left|\widehat{f}\left(\omega_{0}-\mu\right)\right| \mathrm{e}^{\phi\left(\omega_{0}-\mu\right)-\phi\left(\omega_{0}\right)}
$$

Like the discrete-time counterpart, the considered phase retrieval problem in the assertion can merely be solved up to rotations. This enables us to define the phase $\phi\left(\omega_{0}\right)$ of the specific point $\omega_{0}$ in the frequency domain arbitrarily. Beginning from this initial phase, we can further determine the complete phase function $\phi$ and hence the FourIER transform $\mathscr{F}[f]$ in a small open interval around $\omega_{0}$ by using the extracted relative phases. Since the unknown FourIER transform $\mathscr{F}[f]$ is the restriction of an entire function as discussed above, the unknown function $\mathscr{F}[f]$ can be uniquely extended from the small interval to the complete frequency domain. Using the inverse FourIER transform, we finally obtain the desired signal $f$.

Theorem 20.5. Let $f$ be a continuous-time signal in $L^{2}$ with compact support. Then the signal $f$ can be uniquely recovered up to a rotation from its FOURIER intensity $|\mathcal{F}[f]|$ and the interference measurements

$$
\left|\mathcal{F}\left[f+\mathrm{e}^{\mathrm{i} \alpha_{1}} \mathrm{e}^{\mathrm{i} \mu \cdot} f\right]\right| \quad \text { and } \quad\left|\mathcal{F}\left[f+\mathrm{e}^{\mathrm{i} \alpha_{2}} \mathrm{e}^{\mathrm{i} \mu \cdot} f\right]\right| \quad(\mu \in M)
$$

where $\alpha_{1}$ and $\alpha_{2}$ are two real numbers satisfying $\alpha_{1}-\alpha_{2} \neq \pi k$ for all integers $k$, and where $M$ is an open neighbourhood around zero.

Proof. Again, the crucial point to verify the assertion is the extraction of the relative phase from the given FourIER intensities

and

$$
\left|\mathcal{F}\left[f+\mathrm{e}^{\mathrm{i} \alpha_{1}} \mathrm{e}^{\mathrm{i} \mu \cdot} f\right]\right|=\left|\widehat{f}(\cdot)+\mathrm{e}^{\mathrm{i} \alpha_{1}} \widehat{f}(\cdot-\mu)\right|
$$

$$
\left|\mathcal{F}\left[f+\mathrm{e}^{\mathrm{i} \alpha_{2}} \mathrm{e}^{\mathrm{i} \mu \cdot} f\right]\right|=\left|\widehat{f}(\cdot)+\mathrm{e}^{\mathrm{i} \alpha_{2}} \widehat{f}(\cdot-\mu)\right|
$$

for every $\mu$ of an open neighbourhood around zero. Letting $\phi$ be the phase function of the unknown Fourier transform $\mathscr{F}[f]$, and following the lines in the proof of the discrete-time counterpart (Theorem 15.11), we can determine the 
values

$$
\mathfrak{R}\left[\mathrm{e}^{\mathrm{i}\left(\phi(\omega-\mu)-\phi(\omega)+\alpha_{1}\right)}\right] \quad \text { and } \quad \mathfrak{R}\left[\mathrm{e}^{\mathrm{i}\left(\phi(\omega-\mu)-\phi(\omega)+\alpha_{2}\right)}\right]
$$

and further the relative phase $\phi(\omega-\mu)-\phi(\omega)$ whenever $\mathcal{F}[f](\omega)$ and $\mathscr{F}[f](\omega-\mu)$ are non-zero by solving the linear equation system.

Based on the extracted relative phases $\phi(\omega-\mu)-\phi(\omega)$, we now can recover the unknown FourIER transform $\mathscr{F}[f]$ of the non-vanishing signal $f$ on a small interval around a suitable point $\omega_{0}$ in the frequency domain as discussed in the previous proof of Theorem 20.4. Since the FourIER transform of $f$ is the restriction of an entire function, we can uniquely extend $\mathscr{F}[f]$ from the interval to the entire frequency domain. Finally, applying the inverse Fourier transform, we obtain the original signal $f$ up to a rotation, which completes the proof.

Remark 20.6. Looking back to the discrete-time counterparts in Theorem 15.9 and Theorem 15.11, we can enforce uniqueness of the phase retrieval problem by employing the interferences with one specific modulation $\mathrm{e}^{\mathrm{i} \mu \cdot} x$ of the unknown discrete-time signal $x$. The key element of the corresponding proof is that we can reconstruct the unknown trigonometric polynomial $\mathscr{F}[x]$ from finitely many samples at suitable equally spaced points in the frequency domain.

In the continuous-time setting, where the trigonometric polynomial $\mathscr{F}[x]$ is replaced by the restriction $\mathscr{F}[f]$ of an entire function, it is no longer possible to recover the unknown function $\mathscr{F}[f]$ completely from finitely or even countably many equally spaced samples. To overcome this difficulty, we determine $\mathscr{F}[f]$ in a small interval in the frequency domain, which allows the reconstruction of the complete function, by increasing the number of the employed modulations. In fact, Theorem 20.4 and Theorem 20.5 require the interference measurements with the modulations $\mathrm{e}^{\mathrm{i} \mu \cdot} x$ for all $\mu$ in an open neighbourhood around zero. In other words, we consider the interferences between the unknown signal and infinitely many modulations. 



\title{
Phase retrieval from FreSNEL magnitudes
}

\begin{abstract}
Up to this point, we have assumed that the intensity in the frequency domain arises from the Fourier transformed signal. Now, we will replace the Fourier transform by the Fresnel transform. In other words, we would like to recover a discrete-time or continuous-time signal from its FrESNEL intensity. Using the close relation between the FrESNEL and the Fourier phase retrieval problem, we will here characterize all occurring ambiguities in analogy to the FourIER setting. Moreover, we will be able to directly transfer most of the approches to reduce the solution set and to enforce uniqueness of the problem. Next, we will establish a convolution theorem for the FrESNEL transform by adapting the results of ZAYED [Zay98] for the fractional FouRIER transform. Based on this convolution theorem, we will finally define a structured function such that the continuous-time FrESNEL phase retrieval problem can be reduced to a completely discrete problem.
\end{abstract}

\section{The Fresnel transform}

In the previous chapters, we have investigated several types of the phase retrieval problem and sought for suitable a priori conditions and additional information about the unknown signal to ensure a unique recovery. Until now we have thereby always assumed that the given magnitudes in the frequency domain arise from the discrete-time or continuous-time FourIER transform. Physically, one can interpret these magnitudes as intensity measurements of a wave on a plane in the far field, see for instance [Goog6, Section 4.3]. In the following sections, we will now consider the question: what happens if we replace the intensity measurements in the far field by intensity measurements on a plane in the near field? Can we transfer our previous findings to the new phase retrieval problem? 
Mathematically, the change from the far field to the near field means that we replace the FOURIER transform model in the phase retrieval problem by the FrESNEL transform model, see for example [Goog6, Section 4.2]. Like the FourIER transform, the FRESNEL transform of a continuous-time signal can be defined in various ways. In order to define the FrESNEL transform in analogy to the employed continuous-time FourIER transform, we here use an adapted version of the definition given by GoRI in [Gor81, p. 294].

Definition 21.1. Let $f$ be a complex-valued continuous-time signal. For the real parameter $\tau$, the (continuous-time) FRESNEL transform of the signal $f$ is defined by

$$
\mathcal{E}_{\tau}[f](\omega):=\widetilde{f}_{\tau}(\omega):=\int_{-\infty}^{\infty} f(t) \mathrm{e}^{\mathrm{i} \tau(\omega-t)^{2}} \mathrm{~d} t
$$

Remark 21.2. If we expand the exponent of $\mathrm{e}^{\mathrm{i} \tau(\omega-t)^{2}}$ in the definition of the FrESNEL transform, then the defining integral can be written as

$$
\mathcal{E}_{\tau}[f](\omega)=\int_{-\infty}^{\infty} f(t) \mathrm{e}^{\mathrm{i} \tau \omega^{2}} \mathrm{e}^{-2 \mathrm{i} \tau \omega t} \mathrm{e}^{\mathrm{i} \tau t^{2}} \mathrm{~d} t
$$

Consequently, we can also define the Fresnel transform directly by using the continuous-time Fourier transform. In this manner, the (continuous-time) FrEsNEL transform can be alternatively defined by

$$
\mathcal{E}_{\tau}[f](\omega)=\mathrm{e}^{\mathrm{i} \tau \omega^{2}} \mathcal{F}\left[\mathrm{e}^{\mathrm{i} \tau \cdot^{2}} f\right](2 \tau \omega)
$$

As a first consequence, the FrEsnel transform is obviously well defined for absolutely integrable and square-integrable functions.

Remark 21.3. As mentioned before, the FrESNEL transform in Definition 21.1 is closely related to the intensity measurements of a wave on a plane in the near field. If the intensity is instead considered on a sphere, then the corresponding measurements in the near field can mathematically be described by the so-called fractional FourIER transform, see [PF94]. This specific integral transform can also be defined by using the usual Fourier transform. More precisely, for $\alpha \notin$ 
$\pi \mathbb{Z}$, the fractional FOURIER transform of a continuous-time signal $f$ is given by

$$
\mathscr{F}_{\alpha}[f](\omega):=c_{\alpha} \mathrm{e}^{-\mathrm{i} \pi \omega^{2} \cot \alpha} \mathscr{F}\left[\mathrm{e}^{-\mathrm{i} \pi \cdot{ }^{2} \cot \alpha} f\right]\left(\frac{\omega}{\sin \alpha}\right)
$$

where $c_{\alpha}:=|\sin \alpha|^{-1 / 2} \mathrm{e}^{\mathrm{i} / 2(\alpha-\pi / 2)}$. Further, for an integer $k$, the fractional FourIER transform is defined by

$$
\mathcal{F}_{2 k \pi}[f]:=f \quad \text { and } \quad \mathcal{F}_{(2 k+1) \pi}[f]:=f(-\cdot),
$$

see for instance [Jam14, Section 3.3.1].

If we compare the FRESNEL transform in (21.1) with the fractional FourIER transform, then we observe that, for $\tau:=-\pi \cot \alpha \neq 0$, the different definitions coincide except for a scaling in the argument of the defining FourIER transform and the prefactor. In the following, we will restrict our observations to the FrEsNEL transform. However, due to the close relation between both transforms, we can immediately transfer all results to the fractional FouRIER transform.

Similarly to the original Fresnel transform defined by GoRI in [Gor81], we can invert the FRESNEL transform $\mathscr{E}_{\tau}$ in Definition 21.1 by applying the FRESNEL transform $\mathscr{E}_{-\tau}$ with the negated paramater $-\tau$. Considering the prefactor in the next statement, we can deduce that, for $\tau \neq 0$, the inverse FrESNEL transform is given by $\mathcal{E}_{\tau}^{-1}=|2 \tau| / 2 \pi \mathcal{E}_{-\tau}$.

Proposition 21.4. Let $f$ be a continuous-time signal in $L^{2}$ and $\tau$ be a real number unequal to zero. Then the FRESNEL transform $\mathcal{E}_{\tau}[f]$ can be inverted by

$$
f=\frac{|2 \tau|}{2 \pi} \mathcal{E}_{-\tau}\left[\mathcal{E}_{\tau}[f]\right]
$$

Proof. The assertion can easily be verified by using the definition of the FresneL transform in (21.1). We obtain

$$
\frac{|2 \tau|}{2 \pi} \mathscr{E}_{-\tau}\left[\mathscr{E}_{\tau}[f]\right]=\frac{|2 \tau|}{2 \pi} \mathrm{e}^{-\mathrm{i} \tau \cdot{ }^{2}} \mathcal{F}\left[\mathcal{F}\left[\mathrm{e}^{\mathrm{i} \tau \cdot{ }^{2}} f\right](2 \tau \cdot)\right](-2 \tau \cdot) .
$$

Further, with the well-known identities

$$
\mathscr{F}[g(2 \tau \cdot)]=\frac{1}{|2 \tau|} \mathscr{F}[g]\left(\frac{\dot{2}}{2 \tau}\right) \quad \text { and } \quad \mathcal{F}^{-1}[f]=\frac{1}{2 \pi} \mathscr{F}[f](-\cdot)
$$


for an arbitrary function $g$ in $L^{2}$, this equation can be simplified to

$$
\frac{|2 \tau|}{2 \pi} \mathscr{E}_{-\tau}\left[\mathscr{E}_{\tau}[f]\right]=\mathrm{e}^{-\mathrm{i} \tau \cdot{ }^{2}} \mathscr{F}^{-1}\left[\mathcal{F}\left[\mathrm{e}^{\mathrm{i} \tau} \cdot{ }^{2} f\right]\right]=f .
$$

Due to the fact that $\mathrm{e}^{\mathrm{i} \tau}{ }^{2} f$ is again a square-integrable function, the FourIER inversion theorem yields the assertion.

Looking back to the previous chapters, most of the time we have considered different variants of the discrete-time phase retrieval in Problem 1.2. If we want to exploit the corresponding findings for the phase retrieval from FrESNEL magnitudes, we have to define an appropriate discrete-time version of the FrEsNEL transform in Definition 21.1. Analogously to the approach in [LP12, Section 3.2.1], where the FRESNEL transform is numerically computed by applying the fast FOURIER transform, we define the discrete-time FRESNEL transform of a discrete-time signal $x$ by

$$
\mathcal{E}_{\tau}[x](\omega)=\mathrm{e}^{\mathrm{i} \tau \omega^{2}} \mathscr{F}\left[\mathrm{e}^{\mathrm{i} \tau \cdot \cdot^{2}} x\right](2 \tau \omega) .
$$

Using the definition of the discrete-time FourIER transform, we can thus determine the discrete-time FrESNEL transform of the signal $x$ by

$$
\mathscr{E}_{\tau}[x](\omega)=\mathrm{e}^{\mathrm{i} \tau \omega^{2}} \sum_{n \in \mathbb{Z}}\left(\mathrm{e}^{\mathrm{i} \tau n^{2}} x[n]\right) \mathrm{e}^{-2 \mathrm{i} \tau \omega n}=\sum_{n \in \mathbb{Z}} x[n] \mathrm{e}^{\mathrm{i} \tau(\omega-n)^{2}} .
$$

Consequently, the discrete-time Fresnel transform can simply be deduced from the continuous-time definition by discretizing the occurring integral.

Unfortunately, we cannot invert the FRESNEL transform $\mathscr{E}_{\tau}[x]$ of the discretetime signal $x$ simply by applying the transform $\mathscr{E}_{-\tau}$ because $\mathscr{E}_{\tau}[x]$ is no longer a discrete-time signal. Reverting the operations in the definition in (21.2), we can nevertheless recover the original discrete-time $x$ from the Fresnel transform $\mathcal{E}_{\tau}[x]$.

Proposition 21.5. Let $x$ be a discrete-time signal with finite support and $\tau$ be a real number unequal to zero. Then the discrete-time FRESNEL transform $\mathcal{E}_{\tau}$ can be inverted by

$$
\mathcal{E}_{\tau}^{-1}\left[\widetilde{x}_{\tau}\right][n]:=\frac{|2 \tau|}{2 \pi} \int_{-\frac{\pi}{|2 \tau|}}^{\frac{\pi}{2 \tau \mid}} \widetilde{x}_{\tau}(\omega) \mathrm{e}^{-\mathrm{i} \tau(\omega-n)^{2}} \mathrm{~d} \omega .
$$


Proof. In order to deduce the inversion formular, we rescale and rearrange the FOURIER representation (21.2) to

$$
\mathrm{e}^{-\mathrm{i} \frac{\omega^{2}}{4 \tau}} \widetilde{x}_{\tau}\left(\frac{\omega}{2 \tau}\right)=\mathscr{F}\left[\mathrm{e}^{\mathrm{i} \tau \cdot{ }^{2}} x\right](\omega) .
$$

Applying the inverse discrete-time FourIER transform, we can recover the components of the signal $x$ by

$$
x[n]=\frac{1}{2 \pi} \mathrm{e}^{-\mathrm{i} \tau n^{2}} \int_{-\pi}^{\pi} \mathrm{e}^{-\mathrm{i} \frac{\omega^{2}}{4 \tau}} \widetilde{x}_{\tau}\left(\frac{\omega}{2 \tau}\right) \mathrm{e}^{\mathrm{i} \omega n} \mathrm{~d} \omega .
$$

With the substitution $\breve{\omega}:=\omega / 2 \tau$, the integral can now be rewritten to

$$
x[n]=\frac{|2 \tau|}{2 \pi} \mathrm{e}^{-\mathrm{i} \tau n^{2}} \int_{-\frac{\pi}{|2 \tau|}}^{\frac{\pi}{\mid 2 \tau}} \mathrm{e}^{-\mathrm{i} \tau \breve{\omega}^{2}} \widetilde{x}_{\tau}(\breve{\omega}) \mathrm{e}^{2 \mathrm{i} \tau \breve{\omega} n} \mathrm{~d} \breve{\omega}=\frac{|2 \tau|}{2 \pi} \int_{-\frac{\pi}{|2 \tau|}}^{\frac{\pi}{2 \tau \mid}} \widetilde{x}_{\tau}(\breve{\omega}) \mathrm{e}^{-\mathrm{i} \tau(\breve{\omega}-n)^{2}} \mathrm{~d} \breve{\omega},
$$

yielding the claimed representation of the inverse discrete-time FrESNEL transform.

\section{Ambiguities of the FrESNEL phase retrieval problem}

With the definitions of the continuous-time and discrete-time FrESNEL transform in Section 21, we are now ready to investigate the phase retrieval problem from Fresnel magnitudes. Different from our procedure above, we start with the continuous-time formulation.

Problem 22.1. The continuous-time FRESNEL phase retrieval problem is the problem of recovering a continuous-time signal $f$ in $L^{2}$ with compact support from its FRESNEL intensity $\left|\mathscr{E}_{\tau}[f]\right|$ for a fixed real number $\tau$ unequal to zero.

To characterize the solutions of this phase retrieval problem, we exploit the close relation between the FrESNEL and FOURIER transform. Using the definition in (21.1), we can hence represent the given FrESNEL intensity by

$$
\left|\mathscr{E}_{\tau}[f](\omega)\right|=\left|\mathcal{F}\left[\mathrm{e}^{\mathrm{i} \tau \cdot{ }^{2}} f\right](2 \tau \omega)\right| .
$$


Except for the additional dilation by $2 \tau$, the given FrESNEL intensity is thus the FourIER intensity of the modulated signal $\mathrm{e}^{\mathrm{i} \tau} \tau^{2} f$. Consequently, we can reduce the considered FrESNEL phase retrieval problem completely to the continuoustime phase retrieval problem from FourIER magnitudes, which gives us full access to our findings in Section 18 and Section 20.

Although the phase retrieval problems from FrESNEL and FourIER magnitudes nearly coincide, the additional modulation by $\mathrm{e}^{\mathrm{i} \tau} \cdot{ }^{2}$ marginally changes the behaviour of the given intensity with respect to a time shift or the conjugation and reflection of the original signal. More precisely, the FrESNEL transform of a shifted and a reflected and conjugated signal is given by

$$
\mathcal{E}_{\tau}\left[f\left(\cdot-t_{0}\right)\right](\omega)=\mathcal{E}_{\tau}[f]\left(\omega-t_{0}\right) \quad \text { and } \quad \mathcal{E}_{\tau}[\overline{f(-\cdot)}](\omega)=\overline{\mathcal{E}_{-\tau}[f](-\omega)}
$$

Here both identities can be verified by a simple substitution.

Hence, the time shift or the reflection and conjugation of a signal $f$ causes a shift or conjugation and reflection of the corresponding Fresnel transform. The reflection and conjugation additionally negates the parameter $\tau$. Consequently, the trivial ambiguities of the FOURIER phase retrieval problem caused by shifts or reflection and conjugation are no valid solutions of the FRESNEL phase retrieval problem. Considering the shift and the reflection and conjugation together with appropriate modulations, we can nevertheless construct very simple ambiguities of Problem 22.1.

Proposition 22.2. Let $f$ be a continuous-time signal in $L^{2}$ with compact support and FRESNEL intensity $\left|\mathscr{E}_{\tau}[f]\right|$. Then

(i) the rotated signal $\mathrm{e}^{\mathrm{i} \alpha} f$ for real $\alpha$

(ii) the shifted and modulated signal $\mathrm{e}^{-2 \mathrm{i} \tau t_{0} \cdot} f\left(\cdot-t_{0}\right)$ for real $t_{0}$

(iii) the reflected, conjugated, and modulated signal $\mathrm{e}^{-2 \mathrm{i} \tau \cdot{ }^{2}} \overline{f(-\cdot)}$

have the same FRESNEL intensity $\left|\varepsilon_{\tau}[f]\right|$.

Proof. Using the Fourier representation of the Fresnel transform in (21.1), we can justify the assertion by transferring the lines in the proof of Proposition 2.1 to the continuous-time setting.

(i) The FrESNEL transform of the rotated signal is obviously given by

$$
\mathcal{E}_{\tau}\left[\mathrm{e}^{\mathrm{i} \alpha} f\right](\omega)=\mathrm{e}^{\mathrm{i} \alpha} \mathcal{E}_{\tau}[f](\omega) .
$$


Since the prefactor $\mathrm{e}^{\mathrm{i} \alpha}$ is a unimodular constant, the FrESNEL intensity of the rotated signal coincides with $\left|E_{\tau}[f]\right|$.

(ii) Using the representation of the FRESNEL transform in (21.1) and completing the square in the exponent of the inner modulations, we can write the FrESNEL transform of the shifted and modulated signal as

$$
\begin{aligned}
\mathscr{E}_{\tau}\left[\mathrm{e}^{-2 \mathrm{i} \tau t_{0} \cdot} f\left(\cdot-t_{0}\right)\right](\omega) & =\mathrm{e}^{\mathrm{i} \tau \omega^{2}} \mathcal{F}\left[\mathrm{e}^{\mathrm{i} \tau \cdot{ }^{2}} \mathrm{e}^{-2 \mathrm{i} \tau t_{0} \cdot} f\left(\cdot-t_{0}\right)\right](2 \tau \omega) \\
& =\mathrm{e}^{\mathrm{i} \tau t_{0}^{2}} \mathrm{e}^{\mathrm{i} \tau \omega^{2}} \mathcal{F}\left[\mathrm{e}^{\mathrm{i} \tau\left(\cdot-t_{0}\right)^{2}} f\left(\cdot-t_{0}\right)\right](2 \tau \omega)
\end{aligned}
$$

Since a shift in the time domain corresponds to a modulation in the frequency domain, we finally obtain

$$
\begin{aligned}
\mathscr{E}_{\tau}\left[\mathrm{e}^{-2 \mathrm{i} \tau t_{0} \cdot} f\left(\cdot-t_{0}\right)\right](\omega) & =\mathrm{e}^{\mathrm{i} \tau t_{0}^{2}} \mathrm{e}^{-2 \mathrm{i} \tau \omega t_{0}} \mathrm{e}^{\mathrm{i} \tau \omega^{2}} \mathcal{F}\left[\mathrm{e}^{\mathrm{i} \tau \tau^{2}} f\right](2 \tau \omega) \\
& =\mathrm{e}^{\mathrm{i} \tau t_{0}^{2}} \mathrm{e}^{-2 \mathrm{i} \tau \omega t_{0}} \mathscr{E}_{\tau}[f](\omega) .
\end{aligned}
$$

Consequently, the Fresnel transform of the shifted and modulated signal coincides with $\mathscr{E}_{\tau}[f]$ except for a rotation and modulation, which leads us to the assertion.

(iii) To determine the FrESNEL transform of the reflected, conjugated, and modulated signal, we exploit the representation in (21.1). In this manner, we obtain

$$
\begin{aligned}
\mathscr{E}_{\tau}\left[\mathrm{e}^{-2 \mathrm{i} \tau \cdot{ }^{2}} \overline{f(-\cdot)}\right](\omega) & =\mathrm{e}^{\mathrm{i} \tau \omega^{2}} \mathscr{F}\left[\mathrm{e}^{-\mathrm{i} \tau \cdot \cdot^{2}} \overline{f(-\cdot)}\right](2 \tau \omega) \\
& =\mathrm{e}^{\mathrm{i} \tau \omega^{2}} \mathscr{F}\left[\overline{\mathrm{e}^{\mathrm{i} \tau(-\cdot)^{2}} f(-\cdot)}\right](2 \tau \omega) .
\end{aligned}
$$

Exploiting that the reflection and conjugation of a signal in the time domain results in the conjugation of the FOURIER transformed signal now yields

$$
\mathcal{E}_{\tau}\left[\mathrm{e}^{-2 \mathrm{i} \tau \cdot \cdot} \overline{f(-\cdot)}\right](\omega)=\mathrm{e}^{\mathrm{i} \tau \omega^{2}} \overline{\mathcal{F}\left[\mathrm{e}^{\mathrm{i} \tau \cdot} f\right](2 \tau \omega)}=\mathrm{e}^{2 \mathrm{i} \tau \omega^{2}} \overline{\mathcal{E}_{\tau}[f](\omega)} .
$$

Taking the absolute value, we observe that the FrEsneL intensities of both signals coincide as conjectured.

Remark 22.3. All three ambiguities in Proposition 22.2 arise from the trivial ambiguities of the underlying phase retrieval problem to recover $\mathrm{e}^{\mathrm{i} \tau} .^{2} f$ from its FourIER intensity. Especially, the modulations for the shifted and the reflected 
and conjugated signal compensate the shift or the reflection and conjugation of the additional modulation $\mathrm{e}^{\mathrm{i} \tau{ }^{2}}$. In analogy to the FourIER phase retrieval problem, we call the three ambiguities in Proposition 22.2 caused by rotations, shifts, and reflection and conjugation together with the appropriate modulations trivial. The remaining ambiguities are non-trivial.

Remark 22.4. Quite different from the phase retrieval problem from FoURIER magnitudes, all three trivial ambiguities of the FrESNEL phase retrieval problem can be easily avoided by assuming that the unknown signal is real-valued and non-negative. The main reason for this behaviour is that the ambiguities caused by time shifts or by reflection and conjugation only occur with an additional modulation, which prevents that the arising ambiguity is again real-valued and hence a valid solution of the restricted problem.

Due to the close relation between the Fresnel and Fourier phase retrieval problem, we can characterize all possible solutions of the continuous-time phase retrieval problem from Fresnel magnitudes (Problem 22.1) similarly to Theorem 18.5.

Theorem 22.5. Let $f$ be a continuous-time signal in $L^{2}$ with compact support. Then each continuous-time signal $g$ in $L^{2}$ with compact support and the same FRESNEL intensity $\left|\varepsilon_{\tau}[g]\right|=\left|\varepsilon_{\tau}[f]\right|$ for a certain $\tau$ is characterized via the LAPLACE transform by

$$
\mathcal{L}\left[\mathrm{e}^{\mathrm{i} \tau \cdot{ }^{2}} g\right](\zeta)=C \zeta^{m} \mathrm{e}^{\zeta \gamma} \prod_{j=1}^{\infty}\left(1-\frac{\zeta}{\eta_{j}}\right) \mathrm{e}^{\frac{\zeta}{\eta_{j}}}
$$

where the absolute value $|C|$ and the imaginary part $\mathfrak{J} \gamma$ of the complex constants $C$ and $\gamma$ coincide for all signals, and where $\eta_{j}$ is chosen from the zero pairs $\left(\xi_{j},-\bar{\xi}_{j}\right)$ of the autocorrelation function A of the signal $\mathrm{e}^{\mathrm{i} \tau} .^{2} f$.

Proof. As seen in (22.1), the continuous-time signal $g$ is a solution of Problem 22.1 if the Fourier intensity $\left|\mathcal{F}\left[\mathrm{e}^{\mathrm{i} \tau} .^{2} g\right]\right|$ coincide with $\left|\mathscr{F}\left[\mathrm{e}^{\mathrm{i} \tau .^{2}} f\right]\right|$. In other words, the function $\mathrm{e}^{\mathrm{i} \tau} .^{2} g$ is a solution of the underlying FourIER phase retrieval problem, which leads us to the assertion by applying Theorem 18.5.

Remark 22.6. The fundamental idea behind Theorem 22.5 is to solve the continuous-time phase retrieval problem to recover the unknown signal $\mathrm{e}^{\mathrm{i} \tau} .^{2} f$ from its FourIER intensity $\left|\mathcal{F}\left[\mathrm{e}^{\mathrm{i} \tau \tau^{2}} f\right]\right|$. Afterwards, we can determine all solutions 
of original FRESNEL phase retrieval problem by multiplying each solution of the underlying Fourier phase retrieval problem with $\mathrm{e}^{-\mathrm{i} \tau} \cdot{ }^{2}$.

Based on this approach, we can now adapt our findings in Section 20 to ensure the uniqueness by employing additional interference measurements to the phase retrieval problem from Fresnel magnitudes. Especially, Proposition 20.1 and Theorem 20.3 for known and unknown reference signals can be transferred without circumstances. Further, since the FRESNEL transform of the interference between the unknown signal $f$ and the modulated version $\mathrm{e}^{i \alpha} \mathrm{e}^{\mathrm{i} \mu \cdot} f$ can be written as

$$
\begin{aligned}
\left|\mathscr{E}_{\tau}\left[f+\mathrm{e}^{\mathrm{i} \alpha} \mathrm{e}^{\mathrm{i} \mu \cdot} f\right]\right| & =\left|\mathcal{F}\left[\mathrm{e}^{\mathrm{i} \tau \cdot{ }^{2}} f+\mathrm{e}^{\mathrm{i} \alpha} \mathrm{e}^{\mathrm{i} \mu \cdot} \mathrm{e}^{\mathrm{i} \tau \cdot{ }^{2}} f\right](2 \tau \cdot)\right| \\
& =\left|\mathcal{F}\left[\mathrm{e}^{\mathrm{i} \tau \cdot{ }^{2}} f\right](2 \tau \cdot)+\mathrm{e}^{\mathrm{i} \alpha} \mathcal{F}\left[\mathrm{e}^{\mathrm{i} \tau \cdot{ }^{2}} f\right](2 \tau \cdot-\mu)\right|
\end{aligned}
$$

by using the FOURIER representation in (21.1), we can also employ Theorem 20.4 and Theorem 20.5 to enforce the uniqueness of the continuous-time FrESNEL phase retrieval problem.

In the remaining part of this section, we consider the discrete-time version of Problem 22.1. In other words, we want to recover an unknown discrete-time signal from the magnitude of its FrESNEL transform. Analogously to the procedure in Chapter I, we here restrict ourselves to the reconstruction of discrete-time signals with finite support.

Problem 22.7. The discrete-time FRESNEL phase retrieval problem is the problem to recover a discrete-time signal $x$ with finite support from its FRESNEL intensity $\left|\mathscr{E}_{\tau}[x]\right|$ for a certain real number $\tau \neq 0$.

Like for the continuous-time problem, we can exploit the close relationship between the FrESNEL and Fourier transform in (21.2). In this manner, we can reduce the discrete-time FRESNEL phase retrieval problem by

$$
\left|\mathcal{E}_{\tau}[x](\omega)\right|=\left|\mathcal{F}\left[\mathrm{e}^{\mathrm{i} \tau \cdot{ }^{2}} x\right](2 \tau \omega)\right|
$$

to the reconstruction of the discrete-time signal $\mathrm{e}^{\mathrm{i} \tau \cdot^{2}} x$ from its FourIER intensity. As discussed in Proposition 22.2, we can transfer the concept of trivial and nontrivial ambiguities by considering the additional modulation $\mathrm{e}^{\mathrm{i} \tau .{ }^{2}}$ appropriately. Moreover, each occurring ambiguity - trivial or non-trivial - can be explicitly characterized in the time domain similarly to Theorem 5.1. 
Theorem 22.8. Let $x$ be a discrete-time signal with finite support and support length $N$. Each discrete-time signal y satisfying $\left|E_{\tau}[y]\right|=\left|E_{\tau}[x]\right|$ can be written in the form

$$
y=\mathrm{e}^{\mathrm{i} \alpha} \mathrm{e}^{-\mathrm{i} \tau} \cdot \sqrt{|a[N-1]| \prod_{j=1}^{N-1}\left|\beta_{j}\right|^{-1}} \cdot\left[\delta_{n_{0}} * \underset{j=1}{*} \underset{*}{*}\left(\delta_{1}-\beta_{j} \delta_{0}\right)\right],
$$

where $\alpha$ is a real number, $n_{0}$ is an integer, $a$ is the autocorrelation signal to $\mathrm{e}^{\mathrm{i} \tau} \cdot{ }^{2} x$ and $\beta_{j}$ is chosen from the zero pairs $\left(\gamma_{j}, \bar{\gamma}_{j}^{-1}\right)$ of the associated polynomial to $\left|\mathscr{F}\left[\mathrm{e}^{\mathrm{i} \tau} \cdot{ }^{2} x\right]\right|^{2}$.

Proof. The characterization in the assertion can be immediately deduced from the underlying FOURIER phase retrieval problem. More precisely, equation (22.3) implies that $y$ is a solution of the considered problem if and only if $\mathrm{e}^{\mathrm{i} \tau} \cdot{ }^{2} y$ is a solution of the phase retrieval problem to recover $\mathrm{e}^{\mathrm{i} \tau} \cdot{ }^{2} x$ from its FoURIER intensity. The other way around, we obtain all solutions of the discrete-time FrESNEL problem by multiplying the solutions of the underlying FouRIER problem in Theorem 5.1 with the exponential $\mathrm{e}^{-\mathrm{i} \tau \cdot^{2}}$, which yields the claimed representation.

Remark 22.9. In the same manner as discussed in Remark 22.6 for the continuous-time FrESNEL phase retrieval problem, we can transfer our findings to enforce uniqueness by exploiting additionally interference measurements like in Chapter IV to the discrete-time Fresnel problem.

Moreover, since the moduli of the discrete-time signals $x$ and $\mathrm{e}^{\mathrm{i} \tau \cdot{ }^{2}} x$ coincide, and the phases only differ by the known modulation $\mathrm{e}^{\mathrm{i} \tau}{ }^{2}$, we can easily adapt our results in Chapter III to the phase retrieval problem from Fresnel magnitudes. In other words, almost every discrete-time signal $x$ with normalized support can be uniquely recovered from its FrESNEL intensity $\left|\mathscr{E}_{\tau}[x]\right|$ and one given absolute value $|x[n]|$ in the time domain up to trivial ambiguities. An analogous observation holds for the recovery of $x$ from its FrESNEL intensity $\left|\mathscr{E}_{\tau}[x]\right|$ and at least two phases $\arg x[n]$.

Unfortunately, we cannot transfer our findings about the non-negativity restriction in Chapter II to the new setting. Here the main obstacle is the additional modulation in the FourIER representation (22.3) of the FRESNEL phase retrieval problem. More precisely, the non-negativity restriction for the solutions of the underlying FouRIER phase retrieval problem in (22.3) corresponds to the assumption that the components $x[n]$ of the unknown signal $x$ in the FrESNEL case possess the phases $-\tau n^{2}$. 


\section{Establishing a Fresnel convolution theorem}

By generalizing our findings for the discrete-time phase retrieval problem to the continuous-time problem in Chapter $\mathrm{V}$, we learned that the phase retrieval problem to recover a continuous-time signal with a certain structure from its FOURIER intensity has exactly the same behaviour as the investigated discrete-time problem. In other words, for structured signals of the form (16.1), we can completely reduce the continuous-time phase retrieval problem to an underlying discretetime problem. Based on this observation, can we find an analogous structure in the FrESNEL setting?

The main reason for the behaviour of the structured functions in the FourIER setting is hidden in the convolutions theorem for the FourIER transform. Following the arguments in Remark 16.4, we can represent each structured function $f$ based on the generator function $\varphi$ in $L^{1}$ or $L^{2}$ by

$$
f:=\sum_{n \in \mathbb{Z}} c[n] \varphi(\cdot-n)=\varphi *\left(\sum_{n \in \mathbb{Z}} c[n] \delta_{n}\right),
$$

where $c$ is a complex-valued sequence with finite support. Since the impulse train on the right-hand side merely consists of finitely many shifts of the DIRAC delta distribution, the convolution is well defined, see for instance [HS94, Section 2.1.3]. With the convolution theorem for the FouRIER transform of distributions, see [HS94, Theorem 3.28], we can now separate the FourIER intensity of the generator function and the impulse train by

$$
|\mathcal{F}[f]|=|\mathcal{F}[\varphi]|\left|\mathcal{F}\left[\sum_{n \in \mathbb{N}} c[n] \delta_{n}\right]\right|=|\widehat{\varphi}||\widehat{c}|,
$$

which leads us to the underlying discrete-time phase retrieval problem to recover the coefficient sequence from its FoURIER intensity $|\widehat{c}|$.

Unfortunately, the above employed convolution $*$ is not compatible with the FRESNEL transform. More precisely, if we restrict ourselves to continuous-time signals in $L^{1}$ or $L^{2}$, where the convolution $f * g$ can be written as the integral

$$
(f * g)(t):=\int_{-\infty}^{\infty} f(s) g(t-s) \mathrm{d} s,
$$

then the FrESNEL transform of the convolved signal $f * g$ is given by the double 
integral

$$
\mathscr{E}_{\tau}[f * g](\omega)=\int_{-\infty}^{\infty} \int_{-\infty}^{\infty} f(s) g(t-s) \mathrm{e}^{\mathrm{i} \tau(\omega-t)^{2}} \mathrm{~d} s \mathrm{~d} t .
$$

Changing the integration order and substituting $\breve{t}:=t-s$ yields

$$
\begin{aligned}
\mathscr{E}_{\tau}[f * g](\omega) & =\int_{-\infty}^{\infty} \int_{-\infty}^{\infty} f(s) g(\breve{t}) \mathrm{e}^{\mathrm{i} \tau(\omega-\breve{t}-s)^{2}} \mathrm{~d} \breve{t} \mathrm{~d} s \\
& =\left(f * \mathcal{E}_{\tau}[g]\right)(\omega)=\left(\mathcal{E}_{\tau}[f] * g\right)(\omega) .
\end{aligned}
$$

Hence, the FrESNEL transform of two convolved functions results in a convolution where one factor is transformed. This behavior is different from the convolution theorem for the FourIER transform, where we obtain the product of the two transformed signals.

Using the identity (23.3) or (22.2), the FrESNEL transform of the structured function in (23.1) is given by

$$
\mathcal{E}_{\tau}[f]=\sum_{n \in \mathbb{Z}} c[n] \mathcal{E}_{\tau}[\varphi](\cdot-n)
$$

In other words, we again obtain a structured function that is based on the transformed generator $\varepsilon_{\tau}[f]$. Thus, the Fresnel transform does not separate the generator function $\varphi$ and the coefficient sequence $c$ as seen in (23.2), and we cannot reduce the continuous-time FRESNEL phase retrieval problem to recover a structured signal of the form (23.1) to a discrete-time problem.

To overcome this difficulty and to transfer our findings about the FoURIER phase retrieval problem for structured signals, we will define an appropriate convolution whose FRESNEL transform is the product of the transformed factors. In other words, we will establish a convolution theorem for the FrESNEL transform. For this purpose, we adapt the convolution defined by ZAYED in [Zay98, p. 102] for the fractional FourIER transform to the FrESNEL transform in Definition 21.1.

Definition 23.1. For the real parameter $\tau$, the convolution $f \star_{\tau} g$ of the complexvalued functions $f$ and $g$ is defined by the integral

$$
\left(f \star_{\tau} g\right)(t):=\mathrm{e}^{-\mathrm{i} \tau t^{2}} \int_{-\infty}^{\infty} f(s) \mathrm{e}^{\mathrm{i} \tau s^{2}} g(t-s) \mathrm{e}^{\mathrm{i} \tau(t-s)^{2}} \mathrm{~d} s .
$$


In analogy to the convolution defined by ZAYED for the fractional FourIER transform, the FRESNEL transform of the convolved signal $f \star_{\tau} g$ results in the product of the transformed factors $\mathscr{E}_{\tau}[f]$ and $\mathscr{E}_{\tau}[g]$. More precisely, the convolution $\star_{\tau}$ fulfils the following convolution theorem.

Theorem 23.2. Let $f, g$, and $f \star_{\tau} g$ be continuous-time signals in $L^{1}$ or in $L^{2}$. The FRESNEL transform of the convolved signal $f \star_{\tau} g$ is given by

$$
\mathcal{E}_{\tau}\left[f \star_{\tau} g\right](\omega)=\mathrm{e}^{-\mathrm{i} \tau \omega^{2}} \mathscr{E}_{\tau}[f](\omega) \mathscr{E}_{\tau}[g](\omega)
$$

Proof. We adapt the proof of the corresponding statement for the fractional Fourien transform by ZAYED in [Zay98, p. 102]. Using the definitions of the continuous-time Fresnel transform and the convolution $\star_{\tau}$ defined in Definition 23.1, we can write $f \star_{\tau} g$ as

$$
\mathscr{E}_{\tau}\left[f \star_{\tau} g\right](\omega)=\int_{-\infty}^{\infty} \mathrm{e}^{-\mathrm{i} \tau t^{2}} \int_{-\infty}^{\infty} f(s) \mathrm{e}^{\mathrm{i} \tau s^{2}} g(t-s) \mathrm{e}^{\mathrm{i} \tau(t-s)^{2}} \mathrm{e}^{\mathrm{i} \tau(\omega-t)^{2}} \mathrm{~d} s \mathrm{~d} t .
$$

After changing the order of integration, the substitution $\breve{t}:=t-s$ yields

$$
\begin{aligned}
\mathscr{E}_{\tau}\left[f \star_{\tau} g\right](\omega) & =\int_{-\infty}^{\infty} \int_{-\infty}^{\infty} f(s) \mathrm{e}^{\mathrm{i} \tau s^{2}} g(\breve{t}) \mathrm{e}^{\mathrm{i} \tau \breve{t}^{2}} \mathrm{e}^{-\mathrm{i} \tau(\breve{t}+s)^{2}} \mathrm{e}^{\mathrm{i} \tau(\omega-\breve{t}-s)^{2}} \mathrm{~d} \breve{t} \mathrm{~d} s \\
& =\int_{-\infty}^{\infty} \int_{-\infty}^{\infty} f(s) g(\breve{t}) \mathrm{e}^{\mathrm{i} \tau\left(\omega^{2}+\breve{t}^{2}+s^{2}-2 \omega \breve{t}-2 \omega s\right)} \mathrm{d} \breve{t} \mathrm{~d} s .
\end{aligned}
$$

By completing the square in the exponent, we can separate the integrations over $\breve{t}$ and $s$ and obtain

$$
\mathscr{E}_{\tau}\left[f \star_{\tau} g\right](\omega)=\mathrm{e}^{-\mathrm{i} \tau \omega^{2}}\left(\int_{-\infty}^{\infty} f(s) \mathrm{e}^{\mathrm{i} \tau(\omega-s)^{2}} \mathrm{~d} s\right)\left(\int_{-\infty}^{\infty} g(\breve{t}) \mathrm{e}^{\mathrm{i} \tau(\omega-\breve{t})^{2}} \mathrm{~d} \breve{t}\right),
$$

which finishes the proof. 
Remark 23.3. Alternatively, the convolution theorem for the FRESNEL transform in Theorem 23.2 can be proven by writing the convolution $f \star_{\tau} g$ as

$$
\left(f \star_{\tau} g\right)(t)=\mathrm{e}^{-\mathrm{i} \tau t^{2}}\left[\left(\mathrm{e}^{\mathrm{i} \tau \cdot{ }^{2}} f\right) *\left(\mathrm{e}^{\mathrm{i} \tau \cdot{ }^{2}} g\right)\right](t)
$$

and using the Fourier representation of the Fresnel transform in (21.1). Moreover, this representation allows us to transfer the properties of the standard convolution $*$ to the modified convolution $\star_{\tau}$. Especially, we can straightforwardly generalize the convolution $\star_{\tau}$ and the corresponding convolution theorem to distributions.

Although we can already use the convolution theorem for the Fresnel transform in Theorem 23.2 to define an appropriate structure that allows the reduction of the corresponding continuous-time phase retrieval problem to a discrete-time problem, we will modify the convolution in Definition 23.1 to avoid the additional modulation $\mathrm{e}^{-\mathrm{i} \tau{ }^{2}}$. For this purpose, we determine the pre-image of the signal $\mathrm{e}^{2 \mathrm{i} \tau \cdot^{2}}$ under the FRESNEL transform and convolve it with $f \star_{\tau} g$.

Lemma 23.4. The inverse FRESNEL transform of the signal $\mathrm{e}^{2 \mathrm{i} \tau} \cdot{ }^{2}$ for $\tau \neq 0$ is given by

$$
\mathcal{E}_{\tau}^{-1}\left[\mathrm{e}^{2 \mathrm{i} \tau \cdot{ }^{2}}\right](t)=\sqrt{\frac{|2 \tau|}{2 \pi}} \mathrm{e}^{\mathrm{i} \frac{\pi}{4} \operatorname{sgn} \tau} \mathrm{e}^{-2 \mathrm{i} \tau t^{2}}
$$

Proof. Using Proposition 21.4 together with the FouRIER representation of the FRESNEL transform in (21.1), we can determine the inverse Fresnel transform of the signal $\mathrm{e}^{2 \mathrm{i} \tau \cdot^{2}}$ by

$$
\mathscr{E}_{\tau}^{-1}\left[\mathrm{e}^{2 \mathrm{i} \tau \cdot{ }^{2}}\right](t)=\frac{|2 \tau|}{2 \pi} \mathscr{E}_{-\tau}\left[\mathrm{e}^{2 \mathrm{i} \tau \cdot{ }^{2}}\right](t)=\frac{|2 \tau|}{2 \pi} \mathrm{e}^{-\mathrm{i} \tau t^{2}} \mathcal{F}\left[\mathrm{e}^{\mathrm{i} \tau \cdot{ }^{2}}\right](2 \tau t) .
$$

Due to the fact that $\mathrm{e}^{\mathrm{i} \tau .^{2}}$ is neither absolutely integrable nor square-integrable, the occurring Fourier transform is not covered by the $L^{1}$ or $L^{2}$ theory. However, if we interpret $\mathrm{e}^{\mathrm{i} \tau} .^{2}$ as generalized function and hence as distribution, the required FOURIER transform is given by

$$
\mathscr{F}\left[\mathrm{e}^{\mathrm{i} \tau \cdot{ }^{2}}\right](\omega)=\sqrt{\frac{2 \pi}{|2 \tau|}} \mathrm{e}^{\mathrm{i} \frac{\pi}{4} \operatorname{sgn} \tau} \mathrm{e}^{-\mathrm{i} \frac{\omega^{2}}{4 \tau}}
$$

see for instance [Mit13, p. 447]. The inverse FrESNEL transform is thus given by

$$
\mathcal{E}_{\tau}^{-1}\left[\mathrm{e}^{2 \mathrm{i} \tau \cdot{ }^{2}}\right](t)=\sqrt{\frac{|2 \tau|}{2 \pi}} \mathrm{e}^{\mathrm{i} \frac{\pi}{4} \operatorname{sgn} \tau} \mathrm{e}^{-2 \mathrm{i} \tau t^{2}} .
$$


Based on our previous observations, we now modify the convolution $\star_{\tau}$ by an additional convolution with the pre-image of the signal $\mathrm{e}^{2 \mathrm{i} \tau} \cdot{ }^{2}$ under the FrESNEL transform.

Definition 23.5. For the real parameter $\tau$, the convolution $f \circledast_{\tau} g$ of the complex-valued functions $f$ and $g$ is defined by

$$
f \circledast_{\tau} g:=\left(f \star_{\tau} g\right) \star_{\tau} \vartheta_{\tau}
$$

where $\vartheta_{\tau}$ is given by

$$
\vartheta_{\tau}(t):=\sqrt{\frac{|2 \tau|}{2 \pi}} \mathrm{e}^{\mathrm{i} \frac{\pi}{4} \operatorname{sgn} \tau} \mathrm{e}^{-2 \mathrm{i} \tau t^{2}}
$$

Although the continuous-time signal $\vartheta_{\tau}$ is neither absolutely integrable nor square-integrable, the defined convolution $\circledast_{\tau}$ is well defined for signals $f$ and $g$ in $L^{1}$ and $L^{2}$ because the signal $\vartheta_{\tau}$ obviously is continuous and bounded. Moreover, the FRESNEL transform of the convolution $f \circledast_{\tau} g$ finally results in the product $\varepsilon_{\tau}[f]$ and $\mathscr{E}_{\tau}[g]$.

Theorem 23.6. Let $f, g$, and $f \circledast_{\tau} g$ be continuous-time signals in $L^{1}$ or in $L^{2}$. The FRESNEL transform of the convolved signal $f \circledast_{\tau} g$ is given by

$$
\mathcal{E}_{\tau}\left[f \circledast_{\tau} g\right]=\mathcal{E}_{\tau}[f] \mathcal{E}_{\tau}[g] .
$$

Proof. The assertion simply follows from Theorem 23.2 and Lemma 23.4. More precisely, the FrESNEL transform of $f \circledast_{\tau} g$ is given by

$$
\mathscr{E}_{\tau}\left[f \circledast_{\tau} g\right](\omega)=\mathscr{E}_{\tau}\left[f \star_{\tau} g \star_{\tau} \vartheta_{\tau}\right](\omega)=\mathrm{e}^{-2 \mathrm{i} \tau \omega^{2}} \mathscr{E}_{\tau}[f](\omega) \mathscr{E}_{\tau}[g](\omega) \mathscr{E}_{\tau}\left[\vartheta_{\tau}\right](\omega) .
$$

Since the FrESNEL transform $\mathscr{E}_{\tau}\left[\vartheta_{\tau}\right]$ cancels with the modulation $\mathrm{e}^{-2 \mathrm{i} \tau \cdot{ }^{2}}$, the desired convolution theorem follows.

Remark 23.7. In order to determine the convolution $\circledast_{\tau}$ of two signals $f$ and $g$, we have to convolve the signal $h:=f \star_{\tau} g$ with $\vartheta_{\tau}$. This additional convolution can be written as

$$
\left(h \star_{\tau} \vartheta_{\tau}\right)(t)=\sqrt{\frac{|2 \tau|}{2 \pi}} \mathrm{e}^{\mathrm{i} \frac{\pi}{4} \operatorname{sgn} \tau} \mathrm{e}^{-\mathrm{i} \tau t^{2}} \int_{-\infty}^{\infty} h(s) \mathrm{e}^{\mathrm{i} \tau s^{2}} \mathrm{e}^{-\mathrm{i} \tau(t-s)^{2}} \mathrm{~d} s
$$


or, by combining the occurring exponentials and using the definition of the continuous-time FourIER transform, as

$$
\left(h \star_{\tau} \vartheta_{\tau}\right)(t)=\sqrt{\frac{|2 \tau|}{2 \pi}} \mathrm{e}^{\mathrm{i} \frac{\pi}{4} \operatorname{sgn} \tau} \mathrm{e}^{-2 \mathrm{i} \tau t^{2}} \mathscr{F}[h](-2 \tau t) .
$$

Consequently, the additional convolution with $\vartheta_{\tau}$ results in a FouRIER transform and a modulation.

Remark 23.8. Since the convolution $\circledast_{\tau}$ in Definition 23.5 is completely based on the convolution $\star_{\tau}$ in Definition 23.1, we can again straightforwardly generalize the convolution $\circledast_{\tau}$ and the corresponding convolution theorem to distributions and discrete-time signals, cf. Remark 23.3.

Remark 23.9. The continuous-time Fresnel transform in Definition 21.1 can also be interpreted as a linear canonical transform. More detailed, for the real parameters $a, b, c, d$ satisfying $a d-b c=1$, the linear canonical transform of a continuous-time signal is defined by the integral

$$
\int_{-\infty}^{\infty} f(t) K_{(a, b, c, d)}(\omega, t) \mathrm{d} t,
$$

where $K$ denotes the kernel of the transform

$$
K_{(a, b, c, d)}(\omega, t):=\frac{1}{\sqrt{2 \pi \mathrm{i} b}} \mathrm{e}^{\frac{1}{2} \mathrm{i}\left[\frac{a}{b} t^{2}-\frac{2}{b} \omega t+\frac{d}{b} \omega^{2}\right]},
$$

see $\left[\mathrm{WRL}^{+}\right.$o9, p. 853 et seq.]. If we now choose the four parameters

$$
a=1, \quad b=\frac{1}{2 \tau}, \quad c=0, \quad \text { and } \quad d=1,
$$

we obtain the FRESNEL transform $\mathscr{E}_{\tau}[f]$ except for a multiplicative constant.

For every possible choice of the four parameters; WEI, RAN, LI, MA, and TAN establish a suitable convolution such that the convolution of two continuoustime signals in the time domain results in the multiplication of the corresponding linear canonical transformed signals in the frequency domain, see $\left[\mathrm{WRL}^{+} \mathrm{og}\right.$, Theorem 1]. In the light of this more general convolution theorem, we can deduce Definition 23.5 and Theorem 23.6 by adapting the results of WeI et al. to the FrESNEL transform defined in Definition 21.1. 


\section{Phase retrieval of structured signals from FreSNEL mag- nitudes}

With the established convolution theorems for the FrESNEL transform, we can now define an appropriate structure for continuous-time signals that allows us to reduce the corresponding continuous-time FRESNEL phase retrieval problem to a discrete-time problem. For this purpose, we determine the convolution between a generator function $\varphi$ in $L^{1}$ or $L^{2}$ and an impulse train whose coefficient sequence $c$ possesses a finite support. Starting with the convolution $\star_{\tau}$ in Definition 23.1 and using the representation in Remark 23.3, we obtain

$$
\begin{aligned}
\varphi \star_{\tau}\left(\sum_{n \in \mathbb{Z}} c[n] \delta_{n}\right) & =\mathrm{e}^{-\mathrm{i} \tau \cdot{ }^{2}}\left[\left(\mathrm{e}^{\mathrm{i} \tau \cdot{ }^{2}} \varphi\right) *\left(\mathrm{e}^{\mathrm{i} \tau \cdot{ }^{2}} \sum_{n \in \mathbb{Z}} c[n] \delta_{n}\right)\right] \\
& =\mathrm{e}^{-\mathrm{i} \tau \cdot{ }^{2}} \sum_{n \in \mathbb{Z}} \mathrm{e}^{\mathrm{i} \tau n^{2}} c[n] \mathrm{e}^{\mathrm{i} \tau(\cdot-n)^{2}} \varphi(\cdot-n) .
\end{aligned}
$$

Similarly to Section 16 , we now define the structured signal with respect to the convolution $\star_{\tau}$ by

$$
f(t)=\mathrm{e}^{-\mathrm{i} \tau t^{2}} \sum_{n \in \mathbb{Z}} \mathrm{e}^{\mathrm{i} \tau n^{2}} c[n] \mathrm{e}^{\mathrm{i} \tau(t-n)^{2}} \varphi(t-n),
$$

where $\varphi$ is an a priori given generator function not equal to zero, $c:=(c[n])_{n \in \mathbb{Z}}$ is a complex-valued sequence with finite support, and $\tau$ is a non-zero real number. Together with the obvious observation that the FrESNEL transform of a shifted DiRAC delta distribution $\delta_{n}$ is given by

$$
\mathcal{E}_{\tau}\left[\delta_{n}\right](\omega)=\mathrm{e}^{\mathrm{i} \tau(\omega-n)^{2}}
$$

and hence coincides with the discrete-time FrESNEL transform of the shifted unit sample signal, the convolution theorem for $\star_{\tau}$ in Theorem 23.2 yields

$$
\mathscr{E}_{\tau}[f]=\mathscr{E}_{\tau}\left[\varphi \star_{\tau}\left(\sum_{n \in \mathbb{Z}} c[n] \delta_{n}\right)\right]=\mathrm{e}^{-\mathrm{i} \tau \cdot{ }^{2}} \mathscr{E}_{\tau}[\varphi] \mathscr{E}_{\tau}[c]
$$

Consequently, the FreSnEL intensity of the structured signal in (24.1) is given by the product

$$
\left|\mathscr{E}_{\tau}[f]\right|=\left|\mathscr{E}_{\tau}[\varphi]\right|\left|\mathscr{E}_{\tau}[c]\right| .
$$

Since the generator function $\varphi$ and hence $\left|\mathscr{E}_{\tau}[\varphi]\right|$ are not constantly zero, and since $\left|\mathscr{E}_{\tau}[c]\right|^{2}=\left|\mathcal{F}\left[\mathrm{e}^{\mathrm{i} \tau \cdot{ }^{2}} c\right]\right|^{2}$ is again a trigonometric polynomial as the autocor- 
relation function of the discrete-time signal $\mathrm{e}^{\mathrm{i} \tau \cdot{ }^{2}} c$, we can reduce the continuoustime phase retrieval problem to recover the structured signal $f$ from its FrESNEL intensity $\left|\varepsilon_{\tau}[f]\right|$ to the discrete-time problem to recover the coefficient sequence $c$ from $\left|E_{\tau}[c]\right|$. The ambiguities of the phase retrieval problem to recover a structured signal of the form (24.1) can thus be characterized by Theorem 22.8 for the discrete-time setting. Moreover, we can directly transfer our findings to enforce uniqueness of the phase retrieval problem for structured functions from FourIER magnitudes in Section 17.

For the second convolution $\circledast_{\tau}$ in Definition 23.5, we obtain similar results. More precisely, the convolution $\circledast_{\tau}$ of a generator function $\varphi$ with an impulse train whose coefficient sequence has a finite support is given by

$$
\begin{aligned}
\varphi \circledast_{\tau}\left(\sum_{n \in \mathbb{Z}} c[n] \delta_{n}\right) & =\varphi \star_{\tau}\left(\sum_{n \in \mathbb{Z}} c[n] \delta_{n}\right) \star_{\tau} \vartheta_{\tau} \\
& =\mathrm{e}^{-\mathrm{i} \tau \cdot{ }^{2}} \sum_{n \in \mathbb{Z}} \mathrm{e}^{\mathrm{i} \tau n^{2}} c[n] \mathrm{e}^{\mathrm{i} \tau(\cdot-n)^{2}}\left(\varphi \star_{\tau} \vartheta_{\tau}\right)(\cdot-n) .
\end{aligned}
$$

Hence the convolution $\circledast_{\tau}$ leads us to structured signals of the form

$$
f(t)=\mathrm{e}^{-\mathrm{i} \tau t^{2}} \sum_{n \in \mathbb{Z}} \mathrm{e}^{\mathrm{i} \tau n^{2}} c[n] \mathrm{e}^{\mathrm{i} \tau(t-n)^{2}}\left(\varphi \star_{\tau} \vartheta_{\tau}\right)(t-n),
$$

where the additional convolution with $\vartheta_{\tau}$ is merely an additional FourIER transform of the generator function $\varphi$, see Remark 23.7. If the continuous-time signal $\varphi \circledast_{\tau} \vartheta_{\tau}$ is absolutely integrable or square-integrable, we can reduce the phase retrieval problem to recover a structured signal $f$ of the form (24.2) from its FrESNEL intensity

$$
\left|\mathscr{E}_{\tau}[f]\right|=\left|\mathscr{E}_{\tau}[\varphi] \mathscr{E}_{\tau}[c]\right|=\left|\mathscr{E}_{\tau}[\varphi]\right|\left|\mathscr{E}_{\tau}[c]\right|
$$

to the discrete-time problem to recover the coefficient sequence $c$.

Although the structured signals of the form (24.1) and (24.2) are a bit cumbersome, they link the continuous-time and discrete-time FreSnEL phase retrieval problem in exactly the same manner as the structured signals of the form (16.1) the corresponding problems in the FourIER setting. Once more, the additional structure of the unknown signal here reduces the usually infinite set of nontrivial ambiguities in the phase retrieval to a finite set. Furthermore, with a similar argumentation as in Remark 16.3, the FRESNEL intensity $\left|\mathscr{E}_{\tau}[f]\right|$ of a structured function of the form (24.1) or (24.2) with a generator function $\varphi$ in $L^{1}$ is completely determined by $2 N-1$ samples at appropriate points in the frequency domain, where $N$ denotes the support length of the coefficient sequence $c$. 


\section{Conclusion and Outlook}

We have started this thesis with a series of questions like: Knowing the magnitude of a Fourier transformed signal, can we uniquely recover this signal? How do the ambiguities of this problem look, and how can we ensure that we find the correct signal? In order to answer these questions, we have surveyed the available literature about the ambiguousness of the one-dimensional phase retrieval problem and have extend the existing theory by further contributions to ensure a unique recovery of the unknown signal.

More precisely, we have given a novel and complete proof to characterize all occurring ambiguities in the discrete-time phase retrieval of complex-valued signal. Here we have obtained an explicit product representation in the frequency domain and a corresponding convolution representation in the time domain. Moreover, we have showed that all ambiguities arise from appropriate convolutions and the rotation, shift, reflection and conjugations of the occurring factors. We have observe that the number of non-trivially different solutions of the phase retrieval problem is bounded by $2^{N-2}$, where $N$ denotes the support length of the original signal. In contrast to other statements in the literature the number of non-trivial solutions depends on the given FOURIER intensity; it can be distinctly smaller than $2^{N-2}$ and in some special cases, the solution is even determined uniquely.

In order to enforce uniqueness of solution, we have investigated the additional a priori condition that the components of the unknown signal are real and nonnegative. Although the assumed non-negativity can avoid the appearance of any ambiguities, uniqueness cannot be ensured in general. More precisely, we have deduced that neither the set of signals that can be uniquely recovered nor the set of signals without a unique reconstruction is negligible.

Based on the observation in [XYC87] that almost every real-valued signal can be uniquely recovered if one of the end points in the time domain is exactly known, we have examined different kinds of additional information about the 
unknown signal in the time domain. Here our novel approaches have shown that almost every complex-valued signal is uniquely determined by its FourIER intensities and one additionally given absolute value in the time domain. Furthermore, we have obtained an analogous statement for two additionally given phases.

Looking back at earlier literature, we have considered the recovery of an unknown signal from the FouRIER intensities of appropriate interference measurements. Here one of our main results have been that almost every pair of two unknown complex-valued signals is uniquely determined by the FoURIER intensities of both signal and of the interference. Giving a new and complete proof for the complex setting, we have been able to improve the corresponding statement in [RDN13], which require the FOURIER intensity of a further interference. Moreover, we have adapted the ideas in [ABFM14, CESV13] to our specific discrete-time phase retrieval problem. In this manner, we have shown that each discrete-time signal with finite support can always be uniquely reconstructed from the FourIER intensities of suitable interferences with a modulation of the unknown signal itself.

In order to extend our investigation to the continuous-time setting, we have considered the recovery of a structured signal from its continuous-time FourIER intensity. Here we have shown that the corresponding phase retrieval problem can be reduced to a discrete-time problem, which has allowed us to transfer our previous findings for the recovery of discrete-time signals to the new setting. Moreover, we have studied the relationship between the discrete-time and continuous-time problem. Transferring the previous approaches to exploit additional interference measures, we have observed that each continuous-time signal with compact support can be uniquely determined by additional interference measurements with an unknown reference or with a modulation of the signal itself.

Finally, we have replaced the Fourier transform by the Fresnel transform. Based on the close relation between both phase retrieval problems, we have transferred most of our previos findings to the FrEsnel setting. Moreover, we have deduced a structured signal, which has allowed us to reduce the corresponding continuous-time FRESNEL phase retrieval to a completely discrete problem.

Looking back at this thesis, we have mainly investigated the question how to ensure the unique recovery of an one-dimensional discrete-time signal from its Fourier intensity. This leads us to some possible starting point for further research. 
One of the most obvious questions is: can we generalize our observations to the multidimensional phase retrieval problem? As mentioned in the introduction, one can here construct all further solutions of the phase retrieval problem to recover a specific signal by factorizing a multivariate algebraic polynomial, see [Hay82]. In view of the fact that the union of all reducible polynomials is a negligible set [HM82], the multidimensional phase retrieval problem is almost always uniquely solvable.

Similarly to the one-dimensional case, one can easily show that the squared FOURIER intensity coincides with the multidimensional autocorrelation function and is hence a multivariate non-negative trigonometric polynomial. Here the question arises: can we always find a signal whose squared FourIER transform coincide with a given non-negative trigonometric polynomial? Does the corresponding phase retrieval problem possesses at least one solution?

In some applications, the considered signal always corresponds to a reducible polynomial, and the uniqueness of the multidimensional phase retrieval problem cannot be generally ensured in this cases, see [Mil9o]. Can we here use or extend our findings in the one-dimensional setting to ensure the uniqueness in the multidimensional case?

As we have seen, the phase retrieval problem for continuous-time signals has a completely different behavior than the discrete-time version. By transferring our results about additional interference measurements to the continuous-time setting, we have done a first step to combine the theory of both settings. Here a natural direction for further investigations is the question whether the remaining results, which are based on additional assumptions in the time domain, can also be generalized to the continuous-time problem, and conversely, whether the existing results for the continuous-time setting can be exploited to achieve uniqueness in the discrete-time phase retrieval problem.

Using the close relation between the FouRIER and FrESNEL transform, we have been able to transfer most of our previous findings to the phase retrieval problem from FrESNEL magnitudes. Based on this observation, we could possibly extend the findings for the FourIER phase retrieval problem to more general transformations.

Finally, some of the a priori conditions and additional information considered in this thesis are inspired by numerical approaches, see for instance [BCLo2, $\mathrm{LBLo}_{2}, \mathrm{SSD}^{+} \mathrm{06}$ ] and references therein. Consequently, we can hope to exploit our observations for a better understanding of the ill-posedness of the underlying problem and the missing stability of the corresponding algorithms. More generally, here the question arises: how can we combine the developed theory with already available numerical methods? How can we exploit our findings to construct efficient and stable algorithms? 



\section{Bibliography}

[ABFM14] Alexeev, Boris ; Bandeira, Afonso S. ; Fickus, Matthew ; Mixon, Dustin G.: Phase retrieval with polarization. In: SIAM fournal on Imaging Sciences 7 (2014), No. 1, pp. 35-66

[Aku56] Aкuтоwicz, Edwin J.: On the determination of tbe phase of a Fourier integral I. In: Transactions of the American Mathematical Society 83 (1956), September, No. 1, pp. $179-192$

[Aku57] Akutowicz, Edwin J.: On the determination of tbe phase of a Fourier integral II. In: Proceedings of the American Mathematical Society 8 (1957), April, No. 2, pp. 234-238

[BBCEo9] Balan, Radu ; Bodmann, Bernhard G. ; CAsazza, Peter G. ; Edidin, Dan: Painless reconstruction from magnitudes of frame coefficients. In: Journal of Fourier Analysis and Applications 15 (2009), No. 4, pp. 488-501

[BCEo6] Balan, Radu ; Casazza, Peter G. ; Edidin, Dan: On signal reconstruction without phase. In: Applied and Computational Harmonic Analysis 20 (2006), No. 3, pp. 345356

[BCLo2] Bauschke, Heinz H. ; Combettes, Patrick L. ; Luke, D. Russell: Phase retrieval, error reduction algorithm, and Fienup variants: a view from convex optimization. In: Journal of the Optical Society of America A 19 (2002), July, No. 7, pp. 1334-1345

[BCM14] Bandeira, Afonso S. ; Chen, Yutong; Mixon, Dustin G.: Phase retrieval from power spectra of masked signals. In: Information and Interference: A Journal of the IMA 3 (2014), June, No. 2, pp. 83-102

[BF96] BARner, Martin ; Flohr, Friedrich: Analysis II. 3., durchgesehene Auflage. Berlin : Walter der Gruyter, 1996 (de Gruyter Lehrbuch)

[BFGR76] Burge, R. E. ; Fiddy, M. A. ; Greenaway, A. H. ; Ross, G.: The phase problem. In: Proceedings of the Royal Society of London. Series A. Mathematical Physical \& Engineering Sciences 350 (1976), pp. 191-212

[BH15] Bodmann, Bernhard G. ; Hammen, Nathaniel: Stable phase retrieval with lowredundancy frames. In: Advances in Computational Mathematics 41 (2015), April, No. 2, pp. 317-331

[Boa54] BoAs, Ralph P. Jr.: Entire Functions. New York : Academic Press, 1954 (Pure and Applied Mathematics 5) 
[BP15a] BeInert, Robert; Plonka, Gerlind: Ambiguities in one-dimensional discrete phase retrieval from Fourier magnitudes. In: Journal of Fourier Analysis and Applications 21 (2015), December, No. 6, pp. 1169-1198. - DOI 10.1007/sooo41-015-9405-2

[BP15b] BeInert, Robert ; PlonkA, Gerlind: Ambiguities in one-dimensional phase retrieval of structured functions. In: PAMM, Proceedings in Applied Mathematics and Mechanics 15 (2015), October, No. 1, pp. 653-654. - DOI 10.1002/pamm.201510316

[Bri85] BRIGGS, William E.: Zeros and factors of polynomials with positive coefficients and protein-ligand binding. In: Rocky Mountain fournal of Mathematics 15 (1985), pp. $75^{-89}$

[BS79] Bruck, Yu. M. ; Sodin, L. G.: On the ambiguity of the image reconstruction problem. In: Optics communications 30 (1979), September, No. 3, pp. 304-308

[Buho3] Buhmann, M. D.: Radial Basis Functions: Theory and Implementations. Cambridge : Cambridge University Press, 2003 (Cambridge Monographs on Applied and Computational Mathematics 12)

[CESV13] CAndès, Emmanuel J. ; Eldar, Yonina C. ; STrohmer, Thomas ; Voroninski, Vladislav: Phase retrieval via matrix completion. In: SIAM fournal on Imaging Sciences 6 (2013), No. 1, pp. 199-225

[CL72] Colombo, Serge ; Lavoine, Jean: Transformations de Laplace et de Mellin : Formulaires. Mode d'utilisation. Paris : Gauthier-Villars, 1972 (Mémorial des sciences mathématiques 169)

[Coh8o] Cohn, Donald L.: Measure Theory. Boston : Birkhäuser, 1980

[Dau92] Daubechies, Ingrid: Ten Lectures on Wavelets. Philadelphia (Pennsylvania) : Society for Industrial and Applied Mathematics, 1992 (CBMS-NSF Regional Conference Series in Applied Mathematics 61)

[DF84] DAInTy, J. C. ; Fiddy, M. A.: The essential role of prior knowledge in phase retrieval. In: Optica Acta: International fournal of Optics 31 (1984), No. 3, pp. 325-330

[DF87] Dainty, J. C. ; Fienup, J. R.: Phase retrieval and image reconstruction for astronomy. In: STARK, Henry (Ed.): Image Recovery: Theory and Application. Orlando (Florida) : Academic Press, 1987, Chapter 7, pp. 231-275

[DM12] Damelin, Stevan B. ; Miller, Willard Jr.: The Mathematics of Signal Processing. Cambridge : Cambridge University Press, 2012 (Cambridge Texts in Applied Mathematics)

[Fan12] FAnNJIANG, Albert: Absolute uniqueness of phase retrieval with random illumination. In: Inverse Problems 28 (2012), No. 7, pp. 20

[FBD83] Fiddy, M. A. ; BRAmes, B. J. ; DAInty, J. C.: Enforcing irreducibility for phase retrieval in two dimensions. In: Optics Letters 8 (1983), February, No. 2, pp. 96-98

[Fej16] FEJÉr, Leopold: Über trigonometrische Polynome. In: Journal für die reine und angewandte Mathematik 146 (1916), Januar, No. 2, pp. 53-82

[Fie78] FiEnUP, J. R.: Reconstruction of an object from the modulus of its Fourier transform. In: Optics Letters 3 (1978), July, No. 1, pp. 27-29 
[FL12] Fischer, Wolfgang ; Lieb, Ingo: A Course in Complex Analysis : From Basic Results to Advanced Topics. Wiesbaden : Vieweg+Teubner, 2012

[FL13] FANNJIANG, Albert ; LiaO, Wenjing: Fourier phasing with phase-uncertain mask. In: Inverse Problems 29 (2013), No. 12, pp. 21

[GKN79] Gellert, Walter (Ed.) ; Kästner, Herbert (Ed.) ; Neuber, Sigfried (Ed.): Lexikon der Mathematik. 2. Auflage. Leipzig : VEB Bibliographisches Institut, 1979

[Goog6] Goodman, Joseph W.: Introduction to Fourier Optics. 2nd Edition. New York : McGraw-Hill, 1996 (McGraw-Hill Series in Electrical and Computer Engineering : Electromagnetics)

[Gor81] GoRI, F.: Fresnel transform and sampling theorem. In: Optics Communications 39 (1981), November, No. 5, pp. 293-297

[GS72] Gerchberg, R. W. ; SAxton, W. O.: A practical algorithm for the determination of phase from image and diffraction plane pictures. In: Optik - International fournal for Light and Electron Optics 35 (1972), No. 2, pp. 237-246

[Hau91] Hauptman, Herbert A.: The phase problem of x-ray crystallography. In: Reports on Progress in Physics 54 (1991), November, No. 11, pp. 1427-1454

[Hay82] HAYEs, Monson H.: The reconstruction of a multidimensional sequence from the phase or magnitude of its Fourier transform. In: IEEE Transactions on Acoustics, Speech, and Signal Processing ASSP-30 (1982), No. 2, pp. 140-154

[HHLO83] Hove, Patrick L. van ; Hayes, Monson H. ; Lim, Jae S. ; Oppenheim, Alan V.: Signal reconstruction from signed Fourier transform magnitude. In: IEEE Transactions on Acoustics, Speech and Signal Processing ASSP-31 (1983), October, No. 5, pp. 1286-1293

[Hil87] Hildebrand, Francis B.: Introduction to Numerical Analysis. 2nd Edition. New York : Dover Publications, 1987

[HLO8o] Hayes, Monson H. ; Lim, Jae S. ; Oppenheim, Alan V.: Signal reconstruction from phase or magnitude. In: IEEE Transactions Acoustics, Speech, and Signal Processing ASSP-28 (1980), No. 6, pp. 672-68o

[HM82] Hayes, Monson H. ; McClellan, James H.: Reducible polynomials in more than one variable. In: Proceedings of the IEEE (Proceedings Letters) 70 (1982), February, No. 2, pp. 197-198

[Hof64] Hofstetter, Edward M.: Construction of time-limited functions with specified autocorrelation functions. In: IEEE Transaction on Information Theory 10 (1964), April, No. 2, pp. 119-126

[HS94] Hoskins, R. F. ; Sousa Pinto, J. J. M.: Distributions, Ultradistributions and Other Generalized Functions. New York : Ellis Horwood Limited, 1994 (Mathematics and its applications)

[Jam14] JAmING, Philippe: Uniqueness results in an extension of Pauli's phase retrieval problem. In: Applied and Computational Harmonic Analysis 37 (2014), November, No. 3, pp. $413-441$

[Kat68] Katznelson, Yitzhak: An Introduction to Harmonic Analysis. New York : John Wiley \& Sons, 1968 
[KH9oa] KIM, Wooshik ; Hayes, Monson H.: Iterative phase retrieval using two Fourier transform intensities. In: Proceedings : ICASSP 9o: 1990 International Conference on Acoustics, Speech and Signal Processing : April 3-6, 199o Vol. 3 IEEE Signal Processing Society, 1990, pp. 1563-1566

[KHyob] Kıм, Wooshik ; Hayes, Monson H.: Phase retrieval using two Fourier-transform intensities. In: Journal of the Optical Society of America A 7 (1990), March, No. 3, pp. 441-449

[KH91] KIm, Wooshik ; Hayes, Monson H.: The phase retrieval problem in X-ray crystallography. In: Proceedings : ICASSP 91 : 1991 International Conference on Acoustics, Speech and Signal Processing : May 14-17, 1991 Vol. 3 IEEE Signal Processing Society, 1991, pp. 1765-1768

[KH93] KIM, Wooshik ; Hayes, Monson H.: Phase retrieval using a window function. In: IEEE Transactions on Signal Processing 41 (1993), March, No. 3, pp. 1409-1412

[KST95] Klibanov, Michael V. ; Sacks, Paul E. ; Tikhonravov, Alexander V.: The phase retrieval problem. In: Inverse Problems 11 (1995), No. 1, pp. 1-28

[LBLo2] Luke, D. Russell ; Burke, James V. ; Lyon, Richard G.: Optical wavefront reconstruction: theory and numerical methods. In: SIAM Review 44 (2002), No. 2, pp. $169-224$

[LeP61] LePage, Wilbur R.: Complex Variables and the Laplace Transform for Engineers. New York : McGraw-Hill Book Company, 1961 (International Series in Pure and Applied Mathematics)

[LP12] LI, Jun-chang ; PICART, Pascal: Digital Holography. London; Hoboken (New Jersey) : ISTE and John Wiley \& Sons, 2012

[LTo8] Langemann, Dirk ; Tasche, Manfred: Phase reconstruction by a multilevel iteratively regularized Gauss-Newton method. In: Inverse Problems 24 (2008), No. 3, pp. 035006(26)

[LTo9] LAngemann, Dirk ; TASche, Manfred: Multilevel phase reconstruction for a rapidly decreasing interpolating function. In: Results in Mathematics 53 (2009), No. 3-4, pp. $333-340$

[Mar77] Markushevich, A. I.: Theory of functions of a complex variable. 2nd Edition. New York : Chelsea Publishing Co., 1977

[Mar14] MARETzke, Simon: A uniqueness result for propagation-based phase contrast imaging from a single measurement. September 2014. - Preprint, arXiv:1409.4794V1

[Milgo] Millane, R. P.: Phase retrieval in crystallography and optics. In: fournal of the Optical Society of America A 7 (1990), March, No. 3, pp. 394-411

[Mit13] Mitrea, Dorina: Distributions, Partial Differential Equations and Harmonic Analysis. New York : Springer, 2013 (Universitext)

[MK87] McBride, A. C. ; KerR, F. H.: On Namias's fractional Fourier transforms. In: IMA Journal of Applied Mathematics 39 (1987), No. 2, pp. 159-175 
[Nam8o] Namias, Victor: The fractional order Fourier transform and its application to quantum mechanics. In: IMA Journal of Applied Mathematics (Fournal of the Institute of Mathematics and its Applications) 25 (1980), March, No. 3, pp. 241-265

[NQL83a] NAwAB, S. Hamid ; QUATIERI, Thomas F. ; Lim, Jae S.: Algorithms for signal reconstruction from short-time Fourier transform magnitude. In: Proceedings : ICASSP 83 : IEEE International Conference on Acoustics, Speech, and Signal Vol. 8 IEEE, 1983, pp. $800-803$

[NQL83b] NawAB, S. Hamid ; QUATIERI, Thomas F. ; Lim, Jae S.: Signal reconstruction from short-time Fourier transform magnitude. In: IEEE Transactions on Acoustics, Speech and Signal Processing ASSP-31 (1983), August, No. 4, pp. 986-998

[Obr63] ОвRеschкоғF, Nikola: Verteilung und Berechnung der Nullstellen reeller Polynome. Berlin : VEB Deutscher Verlag der Wissenschaften, 1963 (Hochschulbücher für Mathematik 55)

[Ort72] Ortega, James M.: Numerical Analysis : A Second Course. New York : Academic Press, 1972 (Computer Science and Applied Mathematics)

[OS89] Oppenheim, Alan V. ; Schafer, Ronald W.: Discrete-Time Signal Processing. Englewood Cliffs (New Jersey) : Prentice Hall, 1989 (Prentice Hall Signal Processing Series)

[PF94] Pellat-Finet, Pierre: Fresnel diffraction and the fractional-order Fourier transform. In: Optics Letters 19 (1994), September, No. 18, pp. 1388-1390

[Pro95] Prony, R.: Essai expérimental et analytique sur les lois de la Dilatabilité des fluides élastiques et sur celles de la Force expansive de la vapeur de l'eau et de la vapeur de l'alkool, á différentes températures. In: Journal de l'École polytechnique 2 (1795), pp. $24^{-76}$

[PYB14] Pohl, Volker ; YANG, Fanny ; Boche, Holger: Phaseless signal recovery in infinite dimensional spaces using structured modulations. In: fournal of Fourier Analysis and Applications 20 (2014), December, No. 6, pp. 1212-1233

[RDN13] RAz, Oren; Dudovich, Nirit ; NADLER, Boaz: Vectorial phase retrieval of 1-D signals. In: IEEE Transactions on Signal Processing 61 (2013), April, No. 7, pp. 1632-1643

[Rem84] Remmert, Reinhold: Funktionentheorie I. Berlin : Springer-Verlag, 1984 (Grundwissen Mathematik 5)

[RSA $\left.{ }^{+} 11\right]$ Raz, O. ; Schwartz, O. ; Austin, D. ; Wyatt, A. S. ; Schiavi, A. ; Smirnova, O. ; Nadler, B. ; Walmsley, I. A. ; Oron, D. ; Dudovich, N.: Vectorial phase retrieval for linear characterization of attosecond pulses. In: Physical Review Letters 107 (2011), September, No. 13, pp. 133902(5)

[Rud7o] Rudin, Walter: Real and Complex Analysis. International Student Edition. London : McGraw-Hill, 1970 (McGraw-Hill Series in Higher Mathematics)

[SC91] Sahinoglou, Haralambos ; CABrera, Sergio D.: On phase retrieval of finite-length sequences using the initial time sample. In: IEEE Transactions on Circuits and Systems 38 (1991), August, No. 8, pp. 954-958

[Sch69] Schwartz, Jacob T.: Nonlinear Functional Analysis. New York : Gordon and Breach. Science Publishers, 1969 (Notes on mathematics and its applications) 
[SS62] Schirokow, P. A. ; Schirokow, A. P.: Affine Differentialgeometrie. Leipzig : B. G. Teubner Verlagsgesellschaft, 1962

[SSD ${ }^{+}$o6] Seifert, Birger ; Stolz, Heinrich ; Donatelli, Marco ; Langemann, Dirk ; TAsche, Manfred: Multilevel Gauss-Newton methods for phase retrieval problems. In: Journal of Physics. A. Mathematical and General 39 (2006), No. 16, pp. 4191-4206

[SW71] SteIn, Elias M. ; WeIss, Guido: Introduction to Fourier Analysis on Euclidean Spaces. Princeton (New Jersey) : Princeton University Press, 1971 (Princeton Mathematical Series 32)

[Tit39] Titchmarsh, E. C.: The Theory of Functions. and Edition. London: Oxford University Press, 1939

[Wal63] WALther, Adriaan: The question of phase retrieval in optics. In: Optica Acta: International fournal of Optics 10 (1963), No. 1, pp. 41-49

[Wan13] WANG, Yang: The phase retrieval problem. International Conference on Approximation Theory and Applications. Hong Kong, May 2013

[WFB81] Wood, J. W. ; Fiddy, M. A. ; Burge, R. E.: Phase retrieval using two intensity measurements in the complex plane. In: Optics Letters 6 (1981), November, No. 11, pp. $514-516$

[WRL ${ }^{+}$o9] WeI, Deyun ; RAN, Qiwen ; LI, Yuanmin ; MA, Jing ; TAN, Liying: A convolution and product theorem for the linear canonical transform. In: IEEE Signal Processing Letters 16 (2009), October, No. 10, pp. 853-856

[XYC87] Xu, Lei ; YAN, Pingfan ; CHANG, Tong: Almost unique specification of discrete finite length signal: from its end point and Fourier transform magnitude. In: Proceedings : ICASSP 87 : IEEE International Conference on Acoustics, Speech, and Signal Vol. 12 IEEE, 1987, pp. 2097-2100

[Yag96] YAgLE, Andrew E.: 1-D and 2-D minimum and non-minimum phase retrieval by solving linear systems of equations. In: The 1996 IEEE International Conference on Acoustics, Speech, and Signal Processing : Conference Proceedings (ICASSP 96) Vol. 3 IEEE Signal Processing Society, 1996, pp. 1692-1694

[Yag98] YAgle, Andrew E.: Phase retrieval from Fourier magnitude and several initial time samples using Newton's formulae. In: IEEE Transactions on Signal Processing 46 (1998), July, No. 7, pp. 2025-2056

[You8o] Young, Robert M.: An Introduction to Nonharmonic Fourier Series. New York : Academic Press, 1980 (Pure and Applied Mathematics)

[Zay98] ZAYED, Ahmed I.: A convolution and product theorem for the fractional Fourier transform. In: IEEE Signal Processing Letters 5 (1998), April, No. 4, pp. 101-103 


\section{Number systems and spaces}

$\begin{array}{ll}\mathbb{C} & \text { complex numbers } \\ C^{0} & \text { continuous functions } \\ L^{1} & \text { absolutely integrable functions } \\ L^{2} & \text { square-integrable functions } \\ \mathbb{N} & \text { natural numbers without zero } \\ \mathbb{N}_{0} & \text { natural numbers including zero } \\ \mathbb{Q} & \text { rational numbers } \\ \mathbb{R} & \text { real numbers } \\ \mathbb{Z} & \text { integers }\end{array}$

\section{Mathematical constants}

$\begin{array}{ll}\mathrm{e} & \text { EuLER's number } \\ \mathrm{i} & \text { imaginary unit } \\ \pi & \text { pi }\end{array}$

\section{Transformations}

$\begin{array}{ll}\mathcal{E}_{\tau} & \text { FRESNEL transform } \\ \mathscr{F} & \text { FOURIER transform } \\ \mathcal{L} & \text { LAPLACE transform }\end{array}$

\section{Variables}

$\begin{array}{ll}A & \text { autocorrelation function (continuous-time) } \\ a & \text { autocorrelation signal } \\ \widehat{a} & \text { autocorrelation function (discrete-time) } \\ F, G & \text { LAPLACE transformed signals } \\ f, g & \text { continuous-time signals } \\ h & \text { reference signal } \\ T, R & \text { trigonometric polynomials }\end{array}$




$\begin{array}{ll}P, Q & \text { algebraic polynomials } \\ N & \text { support length } \\ x, y & \text { discrete-time signals } \\ B, \Gamma & \text { corresponding zero sets (discrete-time) } \\ \beta_{j}, \gamma_{j} & \text { corresponding zeros (discrete-time) } \\ \zeta_{K} & \text { Kth root of unity } \\ \kappa & \text { chart of a manifold } \\ \Xi & \text { corresponding zero set (continuous-time) } \\ \xi_{j}, \eta_{j} & \text { corresponding zeros (continuous-time) } \\ \phi, \psi & \text { phase functions } \\ \varphi & \text { generator function of a structured signal }\end{array}$

\section{Functions and Operators}

arcsin, arccos $\arg$

det

$\mathfrak{I}$

$\boldsymbol{J}_{F}$

R

$S_{\ell}$

$\sin , \cos$

sgn

$\delta_{0}, \delta_{n_{0}}$

$\delta_{0 n}$

$\lambda$

$\sigma_{\ell}$

\section{Miscellanea}

$|\cdot|$
$\sim$
$\sim$
$*$
$\star, \circledast$

\author{
inverse trigonometric functions \\ argument \\ determinant \\ imaginary part \\ JACOBI matrix of $F$ \\ real part \\ elementary symmetric polynomials \\ trigonometric functions \\ sign function \\ DirAc delta distribution or unit sample signal \\ Kronecker delta \\ LEBESGUE measure \\ modified elementary symmetric polynomials
}


$-\mathbf{A}-$

absolute value of

- arbitrary point, 88-95, $172 \mathrm{f}$.

- end point, $82-88$

- entire signal, 95-100

additional conditions and data

- frequency domain

- - known reference, 127-138, 174, 190

- - modulated signal, 149-161, 176f., $195 f$.

- - unknown reference, 138-149, 175, 192

- non-negativity, 49-80

- time domain

- - moduli, 81-100, $172 \mathrm{f}$.

- - phases, 100-123, 173

-- signal values, $124 \mathrm{f}$.

algebraic variety (uniqueness condition)

- for additional moduli, 91

- for additional phases, 103f., 112

ambiguity

- characterization

- - FOURIER phase retrieval, 29f., 35, 165, 182

- - Fresnel phase retrieval, 206, 208

- common trivial, 139

- non-negative, $64-72$

- non-trivial, $12-20$

- number of, 4 iff.

- representation by

- - convolution, 35, 38, 167f., 208

- - product, 165, 182, 184, 206

- trivial, 12-20, 23, 204

ambiguousness by using

- interference measurement

- - known reference, $135^{-138}$
- - unknown reference, 145, 147ff.

- moduli of entire signal, 98

- non-negativity, 80, 171

- phases of entire signal, 123

antipalindromic, conjugate, 25

a priory conditions and data

- frequency domain

- - known reference, 127-138, 174, 190

- - modulated signal, 149-161, 176f., $195 \mathrm{f}$.

- - unknown reference, 138-149, 175, 192

- non-negativity, 49-80

- time domain

- - moduli, 81-100, $172 \mathrm{f}$.

- - phases, 100-123, 173

- - signal values, $124 \mathrm{f}$.

argument of

- entire signal, $122 f$.

- one arbitrary point, 101-109

- two arbitrary points, 109-122, 173

associated polynomial, 24

- corresponding zero set, 24-29

- factorization, 29-32

autocorrelation

- polynomial, 24

- signal and function, 2of., 181

$$
-\mathbf{B}-
$$

B-Spline, centred linear, 164

BOREL's theorem, 184

$$
-\mathbf{C}-
$$

centred linear B-spline, 164

characterization solution set 
- Fourier phase retrieval

- - entire functions, 182

- - frequency domain, 29f., 184

- - structured signals, 165

- - time domain, 35, 38, $167 f$.

- Fresnel phase retrieval, 206, 208

- Hofstetter, 182

coefficient sequence, 164

common trivial ambiguity, 139

condition

- ambiguousness by using

- - additional moduli, 82, 89, 91

- - additional phases, 102, 109, $111 \mathrm{f}$.

- LAgrange, 170

- non-negativity, 49-8o

- uniqueness by using

- - additional moduli, 85, 91

- - additional phases, 103f., 112

conjugate

- antipalindromic polynomial, 25

- palindromic polynomial, 24

- symmetric, 20

conjugation and reflection, 12-16, 37, 204

constraint

- corresponding zero set, $49 f$.

- last zero pair, 57, 61, 69

continuity of

- corresponding zeros, $72 \mathrm{f}$.

- solution set, 74-77

continuous-time

- autocorrelation, 181

- characterization solution set, 182, 206

- convolution, 209

- Fourier intensity, 164, 181

- Fourier phase retrieval problem, 164, 179

- Fourier transform, 164

- Fresnel intensity, 203

- Fresnel phase retrieval problem, 203

- Fresnel transform, 200

- interference measurements

- - known reference, 190

- - modulated signal, $195 \mathrm{f}$.

- - unknown reference, 192

- inverse FrESNEL transform, 201

- LAPLACE transform, 179

- phase retrieval problem

- - Fourier magnitudes, 164, 179

- - Fresnel magnitudes, 203
- signal, 163

convolution

- adapted, 210, 213

- continuous-time, 209

- discrete-time, 16

- representation, 16f., 35, 38, 167f., 208

convolution theorem

- Fourier transform, 16

- FrESnel transform, 211, 213

corresponding

- polynomial, 24

- zero set, 37

- - extension, 65-68

-- modified, 83,88

- - reflection, 188

- - restriction, $49 \mathrm{f}$.

- - structured signal, 187

$-\mathbf{D}-$

data, additional

- frequency domain

- - known reference, 127-138, 174, 190

- - modulated signal, 149-161, 176f., 195 f.

- - unknown reference, 138-149, 175, 192

- time domain

- - moduli, 81-10o, $172 \mathrm{f}$.

- - phases, 100-123, 173

- - signal values, $124 \mathrm{f}$.

derivative of associated polynomial, 26

Descartes's rule of signs, 55

Dirac distribution, 167

DiRAC signal, 34

discrete-time

- autocorrelation, 2of.

- characterization solution set

- - Fourier phase retrieval, 29f., 35

- - Fresnel phase retrieval, 208

- convolution, 16

- convolution theorem, 16

- discretization FOURIER domain, 22

- equivalent problem, 23

- FourIER intensity, 12, 21

- Fourier phase retrieval problem, 12

- Fourier transform, 10

- Fresnel intensity, 207

- Fresnel phase retrieval problem, 207

- Fresnel transform, 202 
- impulse, 34

- inverse FOURIER transform, 12

- inverse FRESNEL transform, 202

- phase retrieval problem

- - Fourier magnitudes, 12

- - Fresnel magnitudes, 207

- signal, 9

discretization in FOURIER domain, 22

$$
-\mathbf{E}-
$$

elementary symmetric function, 56, 68

enforcing uniqueness

- additional moduli

- - arbitrary point, 95, $172 \mathrm{f}$.

- - end point, 87

- - entire signal, 96

- additional phases

- - entire signal, 123

- - one arbitrary point, 108

- - two arbitrary points, 121f., 173

- additional signal values, $124 \mathrm{f}$.

- interference measurements

- - known reference, 128, 131, 174, 190

- - linear phase reference, 134

- - modulated signal, 152, 157, 159, 176f., $195 f$.

- - unknown reference, 139, 175, 192

entire function, 18o, 182

equivalent problem, 23

- characterization solution set, $29 f$.

- solvability, 32

existence phase retrieval problem with

- several non-negative solutions, 77

- unique non-negative solutions, 78

exponential type, exponential function of, 180

extension, zero set, 59-63, 65-68

$$
-\mathbf{F}-
$$

factorization theorem, HADAMARD's, 182 finite support, 10

fixed support, 14

formulae VIETA's, 56

formulation

- continuous-time problem, 163f., 179, 203

- discrete-time problem, 9-12, 207
FOURIER convolution, 16, 209

FOURIER convolution theorem, 16

FOURIER domain

- characterization solution set, $29 f$.

- discretization, 22

- equivalent problem, 23

- linear phase, 133

- solvability, 32

- trivial ambiguity, 23

FOURIER intensity, 12, 21, 164, 181

FOURIER phase retrieval problem

- continuous-time, 164, 179

- discrete-time, 12

FOURIER transform

- continuous-time, 164

- discrete-time, 10

- fractional, 201

- inverse discrete-time, 12

- linear phase, 133

- unit sample, 34

fractional FOURIER transform, 201

frequency domain

- characterization solution set, $29 f$.

- discretization, 22

- equivalent problem, 23

- linear phase, 133

- solvability, 32

- trivial ambiguity, 23

Fresnel convolution, 210, 213

Fresnel convolution theorem, 211, 213

Fresnel intensity, 203, 207

FRESNEL phase retrieval problem

- continuous-time, 203

- discrete-time, 207

FRESNEL transform

- continuous-time, 200

- convolution, 210, 213

- discrete-time, 202

- inverse continuous-time, 201

- inverse discrete-time, 202

function

- autocorrelation, 2of., 181

- elementary symmetric, 56, 68

- entire, 18o, 182

- generator, 164

- LAgrange, 170

- structured, 164, $215 f$. 


$$
-\mathbf{G}-
$$

generalized polarization identity, 150 generator function, 164

$$
-\mathbf{H}-
$$

HADAMARD's factorization theorem, 182 HOFSTETTER, characterization, 182 HuRwitz

- polynomial, 56

- signal, 64

$$
-\mathbf{I}-
$$

identity, polarization, 150

impulse, 34

infinite product, 182, 184

information, additional

- frequency domain

- - known reference, 127-138, 174, 190

- - modulated signal, 149-161, 176f., 195f.

- - unknown reference, 138-149, 175, 192

- time domain

- - moduli, 81-100, $172 \mathrm{f}$.

- - phases, 100-123, 173

- - signal values, $124 \mathrm{f}$.

intensity

- Fourier, 12, 21, 164, 181

- Fresnel, 203, 207

interference with

- known reference signal, 127-138, 174, 190

- linear phase reference signal, 134

- modulated signal, 149-161, 176f., $195 \mathrm{f}$.

- unknown reference signal, 138-149, 175,

$$
192
$$

inverse

- continuous-time

- - FrESNEL transform, 201

- discrete-time

- - FoURIER transform, 12

- - Fresnel transform, 202

$$
-\mathbf{K}-
$$

known reference signal, 127-138, 174, 190

$$
-\mathbf{L}-
$$

LAGRANGE condition, 170

LAGRANGE function, 170

LAPLACE transform

- continuous-time, 179

- discrete-time, 188

last zero pair

- positivity constraint, 57, 61

- reflected constraint, 69

length, support, 2of., 36

linear

- B-spline, 164

- phase, 133

locus (uniqueness condition)

- for additional moduli, 91

- for additional phases, 103f., 112

$$
-\mathbf{M}-
$$

magnitude of

- arbitrary point, 88-95, 172f.

- end point, 82-88

- entire signal, 95-100

manifold (uniqueness condition), 85

MerCedes-Benz polarization identity, 150

method, PRONY's, 153

modified zero set, 83,88

modulated signal, 149-161, 176f., $195 f$.

modulus of

- arbitrary point, 88-95, $172 f$.

- end point, 82-88

- entire signal, 95-100

$$
-\mathbf{N}-
$$

non-negative

- signal, 49-53

- solution, $64-72$

- trigonometric polynomial, $22 \mathrm{f}$.

non-trivial ambiguity, 12-16, 20

non-trivial ambiguousness

- interference measurement

- - known reference, $135-138$

- - unknown reference, 145, $147 \mathrm{ff}$.

- moduli of entire signal, 98

- non-negativity, 8o, 171

- phases of entire signal, 123 
non-trivial solution, 16 non-uniqueness by using

- interference measurement

- - known reference, $135^{-138}$

- - unknown reference, $145,147 \mathrm{ff}$.

- moduli of entire signal, 98

- non-negativity, 8o, 171

- phases of entire signal, 123

normalized support, 14

number of non-trivial ambiguities, $41 \mathrm{ff}$.

$$
-\mathbf{P}-
$$

PALEy-Wiener's theorem, 180

palindromic, conjugate, 24

phase, linear, 133

phase of

- entire signal, $122 f$.

- one arbitrary point, 101-109

- two arbitrary points, 109-122, 173

phase retrieval problem

- additional signal information, 81-125

- continuity, 72-80

- continuous-time, 179, 203

- discrete-time, 12, 207

- equivalent, 23

- Fourier magnitudes, 12, 164, 179

- frequency domain, 20

- Fresnel magnitudes, 203, 207

- non-negative signals, 49-8o

- structured signals, 164

- trivial and nontrivial ambiguities, 12-20

polarization identity, 150

polynomial

- associated, 24

- conjugate

- - antipalindromic, 25

- - palindromic, 24

- Hurwitzian, 56

- positive, $54 \mathrm{f}$.

- trigonometric, 10, 22f.

positive polynomial, $54 \mathrm{f}$.

product, infinite, 182,184

product representation, 29f., 165, 182, 184, 206

Prony's method, 153

$$
-\mathbf{R}-
$$

real-valued

- signal, $32 \mathrm{f}$.

- trigonometric polynomial, 22

recovery, unique

- additional moduli

- - arbitrary point, 95, $172 \mathrm{f}$.

- - end point, 87

- - entire signal, 96

- additional phases

- - entire signal, 123

- - one arbitrary point, 108

- - two arbitrary points, 121f., 173

- additional signal values, $124 \mathrm{f}$.

- interference measurements

- - known reference, 128, 131, 174, 190

- - linear phase reference, 134

- - modulated signal, 152, 157, 159, 176f., $195 \mathrm{f}$.

- - unknown reference, 139, 175, 192

reference

- known signal, 127-138, 174, 190

- linear phase signal, 134

- modulated signal, 149-161, 176f., $195 \mathrm{f}$.

- unknown signal, 138-149, 175, 192

reflection and conjugation, 12-16, 37, 204

related polynomial, 24

- corresponding zero set, $24-29$

- factorization, 29-32

relationship between problems, $187 \mathrm{ff}$.

representation by

- convolution, 16f., 35, 38, 167f., 208

- product, 29f., 165, 182, 184, 206

restriction by

- additional moduli

- - arbitrary point, 89, 91

- - end point, 82

- additional phases

- - one arbitrary point, 102

- - two arbitrary points, 109, 111f.

restriction of corresponding zero set, $49 \mathrm{f}$.

RIEMANN sphere, 58

root of

- trigonometric polynomial, 24-34

- unity, 150

rotation, 12f., 204

rule of signs, 55 


$$
-\mathbf{S}-
$$

SARD's theorem, 86 sequence, coefficient, 164 shift, 12f., 204

signal

- autocorrelation, 2of., 181

- continuous-time, 163

- Dirac, 34

- discrete-time, 9

- Hurwitzian, 64

- length, 21, 36

- linear phase, 133

- non-negative, 49-53

- real-valued, $32 f$.

- reflected and conjugated, 12-16, 204

- rotated, 12f., 204

- shifted, 12f., 204

- structured, 164, 215f.

- unit sample, 34

solution

- characterization

- - Fourier phase retrieval, 29f., 35, 38, 165, 167f., 182, 184

- - Fresnel phase retrieval, 206, 208

- continuity, 74-77

- convolution representation, $16 f$.

- non-negative, $64^{-72}$

- non-trivial, $12-20$

- number of, 4 1ff.

- real-valued, 32f., 42

- representation by

- - convolution, 35, 38, 167f., 208

- - product, 165, 182, 184, 206

- trivial, 12-20, 204

solvability of phase retrieval problem, 32

sphere, RIEMANN, 58

squared FOURIER intensity, 21, 181

structured signal, 164, 215f.

- additional moduli, $172 \mathrm{f}$.

- additional phases, 173

- characterization solution set, 165

- corresponding zero set, 187

- interference measurements

- - known reference, 174

- - modulated signal, $176 \mathrm{f}$.

- - unknown reference, 175

- non-negativity, 171
- phase retrieval problem, 164 submanifold (uniqueness condition), 85

support

- finite, 10

- length, 2of., 36

- normalized, 14

symmetric

- conjugate, 20

- elementary, 56, 68

$$
-\mathbf{T}-
$$

theorem of

- Borel, 184

- HADAMARD, 182

- Paley-Wiener, 180

- SARD, 86

time domain

- additional information, 81-125

- convolution representation, 16f., 35, 38, 167f., 208

time shift, 12f., 204

trigonometric polynomial, 10, $22 \mathrm{f}$.

trigonometric root polynomial, 24-34

trivial ambiguity, 12-20, 23, 139, 204

two-sided LAPLACE transform, 179

$$
-\mathbf{U}-
$$

uniqueness by

- additional moduli

- - arbitrary point, 95, $172 \mathrm{f}$.

- - end point, 87

- - entire signal, 96

- additional phases

- - entire signal, 123

- - one arbitrary point, 108

- - two arbitrary points, 121f., 173

- additional signal values, $124 \mathrm{f}$.

- interference measurements

- - known reference, 128, 131, 174, 190

- - linear phase reference, 134

- - modulated signal, 152, 157, 159, 176f., $195 f$.

- - unknown reference, 139, 175, 192

- non-negativity, 80, 171

uniqueness condition

- for additional moduli, 85, 91 
- for additional phases, 103f., 112 unit sample signal, 34

unknown reference signal, 138-149, 175, 192

$$
-\mathrm{V}-
$$

variety (uniqueness condition)

- for additional moduli, 91

- for additional phases, 103f., 112

VietA's formulae, 56

$$
-\mathbf{Z}-
$$

zero locus (uniqueness condition)

- for additional moduli, 91

- for additional phases, 103f., 112

zero set

- associated polynomial, 24-29

- corresponding, 37

- extension, 59-63, 65-68

- modified, 83, 88

- reflection, 188

- restriction, $49 \mathrm{f}$.

- structured signal, 187 



\section{Curriculum vitae}

Robert BEINERT, M.Sc.

Address

Institut für Numerische und Angewandte Mathematik

Georg-August-Universität Göttingen

Lotzestraße 16-18

37083 Göttingen, Germany

Office $\quad+4955139-4515$

Email r.beinert@math.uni-goettingen.de

Homepage http://num.math.uni-goettingen.de/ rbeiner

\section{Personal Details}

Date of birth $\quad 11$ February 1986

Place of birth Malchin, Germany

Nationality German

\section{Academic Education}

Since 05/2013 Doctoral study programme: Mathematical Sciences

at the Georg-August-Universität Göttingen

Advisor: Prof. Dr. G. PlONKA-Hoch

04/2011-03/2013 Masters study programme: Mathematics

at the Georg-August-Universität Göttingen

Area of specialisation: Numerical and Applied Mathematics 
Minor field of study: Computer Sciences

Standard period of study: 4 semesters

Master thesis: Multilevel Gauß-Newton-Methoden zur Phasenrekonstruktion

Advisor of master thesis: Prof. Dr. G. PlonkA-HocH

Degree: Master of Science passed with distinction

Overall grade: very good (1.0)

09/2007-02/2011 Bachelor study programme: Applied Mathematics

at the Fachhochschule Flensburg

Standard period of study: 7 semesters

Bachelor thesis: Entwicklung und Anwendung eines Verfahrens zur Kalibration von Flächendetektoren unter Berücksichtigung von Nichtlinearitäten

Advisor of bachelor thesis: Prof. Dr. M. KRÄTZsChMAR

Degree: Bachelor of Science

Overall grade: very good (1.0)

Internship

$08 / 2010-02 / 2011$

\section{Internship and bachelor thesis}

at the Deutsches Elektronen-Synchrotron DESY, Hamburg

Department: Electronics Development Group, FEC - Microelectronics

Advisor: Prof. Dr. M. KrätzschmaR and Dr. K. HANSEN

\section{Alternative civilian service}

$10 / 2005-07 / 2010$

Alternative civilian service

at the Kolping Familienferienwerk Salem e.V.

\section{School and vocational education}

09/2005-07/2006 Technical secondary school: Computer Sciences at the Berufliche Schule Mecklenburg-Strelitz

08/2002-07/2005 Apprenticeship: Central heating and ventilation sys-

tem engineer (Zentralheizungs- und Lüftungsbauer) at the Wärmeversorgung Stavenhagen $\mathrm{GmbH}$

Until 06/2002 Secondary school at the Verbundene Haupt- und Realschule Ivenack 


\section{Research Experience}

Since 10/2015 Member of DFG Research Training Group (GRK 2088) Discovering structure in complex data: Statistics meets Optimization and Inverse Problems

07/2015-09/2015 Scientific assistant at the Institut für Numerische und Angewandte Mathematik, Georg-August-Universität Göttingen

05/2013-06/2015 Member of Project C11 Fresnel wavelets for coherent diffractive imaging within the DFG Collaborative Research Center (SFB 755) Nanoscale Photonic Imaging

05/2008-08/2010 Student assistent at the Institut für Angewandte Mathematik, Fachhochschule Flensburg

\section{Teaching Experience}

$2015 / 16$

$2014 / 15$

2014

$2013 / 14$

2010
Lecture assistance and tutorial: Image and Geometry Processing III, GEorG-August-Universität Göttingen

Tutorial assistance: Image and Geometry Processing I, Georg-August-Universität Göttingen

Tutorial: Inverse Problems II, Georg-August-Universität Göttingen

Lecture and tutorial: Methoden der Numerischen Mathematik, Georg-August-Universität Göttingen

Additional tutorials: Mathematik 2.1 für Energie- und Umweltmanagement, Fachhochschule Flensburg

\section{Participation in Conferences and Workshops}

2015

2014
GAMM 86th Annual Scientific Conference (talk), 23-27 March in Lecce, Italy

SFB 755 Winter School 2015, Nanoscale Photonic Imaging, 15-16 January in Teistungen, Germany

Mathematical Signal Processing and Phase Retrieval (talk), 1-3 September in Göttingen, Germany

Mecklenburger Workshop: Approximationsmethoden und schnelle Algorithmen (talk), 17-20 March in Hasenwinkel, Germany 
24. Rhein-Ruhr-Workshop (talk), 31 January - 1 February in Bestwig, Germany

2013

SFB 755 and SFB 937 Autumn School, 1-5 September in Wildbad Kreuth, Germany

\section{Additional Talks}

2015

Technische Universität Kaiserslautern, Research group: Image Processing, 13 October

Universität Osnabrück, Research group: Applied Analysis, 27 January

\section{Publications}

[BP15b] Beinert, Robert ; PlonkA, Gerlind: Ambiguities in one-dimensional phase retrieval of structured functions. In: PAMM, Proceedings in Applied Mathematics and Mechanics 15 (2015), October, No. 1, pp. 653-654. - DOI 10.1002/pamm.201510316

[BP15a] Beinert, Robert ; Plonka, Gerlind: Ambiguities in one-dimensional discrete phase retrieval from Fourier magnitudes. In: fournal of Fourier Analysis and Applications 21 (2015), December, No. 6, pp. 1169-1198. DOI 10.1007/sooo41-015-9405-2 